1990 State-by-State Assessment of Low-Level Radioactive Wastes Received at Commercial Disposal Sites

National Low-Level Waste Management Program

Ronald L. Fuchs

Kimberly Culbertson-Arendts

September 1991 


\section{Available from}

Superintendent of Documents U.S. Government Printing Office

Post Office Box 37082

Washington, D.C. 20013-7982

and

National Technical Information Service

Springfield, VA 22161 
DOE/LLW-132

Distribution Category: UC-512

DOE/LIJW- -132

DE92 000224

\title{
1990 STATE-BY-STATE ASSESSMENT OF LOW-LEVEL RADIOACTIVE WASTES RECEIVED AT COMMERCIAL DISPOSAL SITES
}

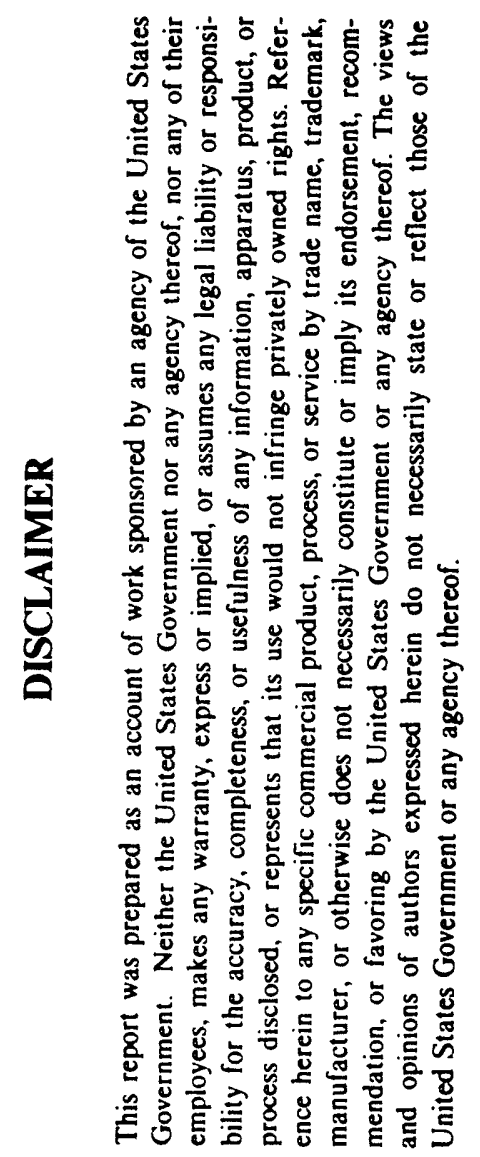

September 1991

\author{
National Low-Level Waste \\ Management Program \\ Idaho Falls, Idaho 83415-3960
}

Prepared by EG\&G Idaho, Inc.

for the U.S. Department of Energy

Under DOE Contract No. DE-AC07-76ID01570

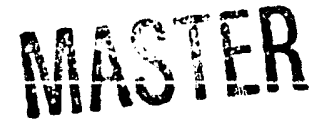




\begin{abstract}
Each year the National Low-Level Waste Management Program publishes a state-by-state assessment report. This annual report provides both national and state-specific disposal data on low-level radioactive wastes. Data in this report are categorized according to disposal site, generator category, waste class, volume, and activity. Included in this report are tables showing a distribution of wastes by state for 1990 and a comparison of waste volumes by state for 1986 through 1990; also included is a list of all commercial nuclear power reactors in the United States as of December 31, 1990. In this year's report, a distinction has been made between low-level radioactive waste shipped directly by generators for disposal and that which was handled by an intermediary.
\end{abstract}

\title{
NOTICE
}

The information presented in this repon is meant to be informative to the reader and provide accurate disposal figures for the commercial low-level radioactive waste being shipped for disposal within the United States. Comments and suggestions as to how this document may better address your needs for information on this subject are welcomed. Comments may be sent to the address listed below:

\author{
Department of Energy \\ National Low-Level Waste Management Program \\ P.O. Box 1625 \\ Idaho Falls, Idaho 83415-3960)
}




\section{CONTENTS}

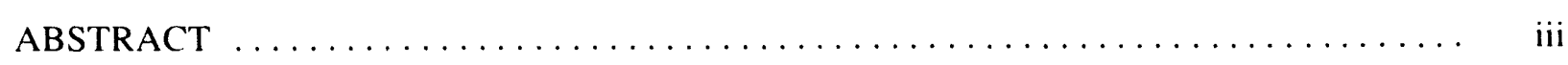

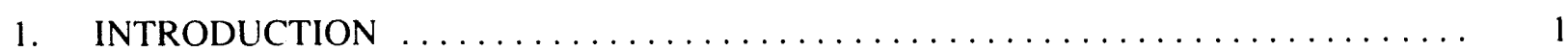

2. 1990 REPORTING METHOD AND SUMMARY $\ldots \ldots \ldots \ldots \ldots \ldots \ldots \ldots \ldots$

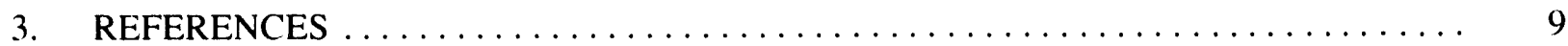

APPENDIX A-1990 LOW-LEVEL WASTE ASSESSMENTS BY COMPACT REGION AND STATE $\ldots \ldots \ldots \ldots \ldots \ldots \ldots \ldots \ldots \ldots \ldots \ldots \ldots \ldots \ldots \ldots \ldots \ldots \ldots \ldots \ldots$

INDEX OF STATES $\ldots \ldots \ldots \ldots \ldots \ldots \ldots \ldots \ldots \ldots \ldots \ldots \ldots \ldots \ldots \ldots \ldots \ldots \ldots \ldots$

APPENDIX B-WASTE VOLUNAE COMPARISON BY STATE AND COMMERCIAL NUCLEAR POWER REACTORS IN THE UNITED STATES $\ldots \ldots \ldots \ldots$ B-1

APPENDIX C-STATE RADIATION PROGRAM REPRESENTATIVES $\ldots \ldots \ldots \ldots \ldots \ldots$ C-1 


\section{STATE-BY-STATE ASSESSMENT OF LOW-LEVEL RADIOACTIVE WASTES RECEIVED AT COMMERCIAL DISPOSAL SITES}

\section{INTRODUCTION}

The National Low-Level Waste Management Program has published twelve annual state-by-state assessment reports. These reports provide both national and state-specific disposal data that assist states in managing low-level radioactive waste ( $L L W)$, as specified in the Low-Level Radioactive Waste Policy Act (Public Law 96-573) and the Low-Level Radioactive Waste Policy Amendments Act of 1985 (LLRWPAA, Public Law 99-240).

Over the past twelve years, every state has generated some quantity of commercial LLW. These wastes are generated from a variety of commercial sources, such as:

- Government and industrial research

- Electrical power generation

- Medical diagnostic and therapeutic services

- Various manufacturing processes.

Before publication of the first state-by-state report in 1979, researchers studied the quantities and radioactivity levels of LLW disposed of in the United States. The first report was designed to assist states in extrapolating accurate estimates of disposal rates by separating wastes into four broad categories:

- Institutional

- Industrial

- Government

- Utility.

In 1983, the categories were further refined by establishing more specific categories such as:

- Academic

- Government

- Industrial

- Medical

- Utility.

Currently, commercial disposal sites report actual volumes disposed for each of these five categories. 
The volume of LLW disposed of at commercial disposal sites exceeded 3.7 million cubic feet in 1980 . This represents the highest reported annual disposal volume of LLW during the history of the state-bystate assessment documents. From 1981 through 1990, the volume of LLW disposed of at commercial disposal sites declined to $1,142,810$ cubic feet.

The 1,142,810 cubic feet of LLW disposed of in 1990 continued the downward trend of low-level radioactive waste disposal and is the lowest reported volume in the report's history. A list of 1990 LLW volumes and radioactivity by state is provided in Table 1 . Only Puerto Rico, which is treated as a state under the LLRWPAA, and the State of Vermont reported no LLW shipments in 1990.

The first state-by-state assessment used several data sources. These included U.S. Nuclear Regulatory Commission records on nuclear power plant waste shipments, ${ }^{1}$ the U.S. Department of Energy Solid Waste Information Management System $s^{2}$ for information on defense wastes shipped to commercial sites, and the University of Maryland (at Baltimore) studies on national institutional waste generation. ${ }^{3,4}$ Since its initial publication, this report has been modified and expanded as more detailed state information became available from the disposal sites. Results from generator surveys and feedback from state officials continue to improve understanding of the sources of LLW and the quality of the data.

Data in this report have been quality checked by comparing data from two sources: (a) monthly disposal activity reports supplied by the regulatory agencies of the sited states of Nevada, South Carolina, and Washington, and (b) shipment manifest data obtained from the three commercial LLW disposal sites for LLW received.

In addition to the verification process, a peer review of :he draft report was conducted by representatives of the individual states' radiation control progra $\mathrm{ms}$. A list of state radiation control program representatives is included in Appendix C. 
Table 1. 1990 Waste distribution by state, as reported by disposal site operators

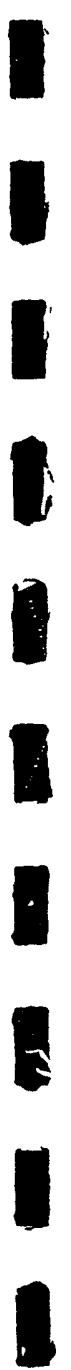

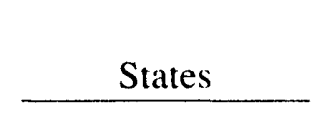

Alabama

Alaska

Arizona

Arkansas

Army Out U.S.

California

Colorado

Connecticut

Delaware

District Of Columbia

Florida

Georgia

Hawaii

Idaho

Illinois

Indiana

Iowa

Kansas

Kentucky

Louisiana

Maine

Maryland

Massachusetts

Michigan

Minnesota $^{\mathrm{a}}$

Mississippi

Missouri

Montana

Nebraska

Nevada

New Hampshire

New Jersey

New Mexico

New York

North Carolina

North Dakota

Ohio

Oklahoma

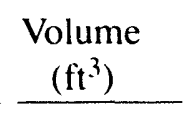

\section{$14,237.55$}

33.51

$27,751.46$

$6,239.42$

35.80

$55,956.54$

2,309.16

$34,138.42$

843.83

538.72

24.587 .01

$55,286.71$

$4,738.86$

40.06

$98,360.33$

$1,956.01$

$5,557.86$

$3,232.54$

$4,616.06$

$11,329.30$

$7,856.78$

$17,037.94$

$40,612.73$

$36,038.53$

$26,864.92$

$5,981.24$

$19,609.59$

195.00

$15,558.87$

313.44

206.44

$52,878.94$

$1,843.54$

$71,284.34$

$48,097.71$

32.90

$24,146.85$

$21,967.85$
Activity

(curies)

$4,857.73$

225.46

246.85

14.39

0.03

$5,537.69$

$1,451.28$

$255,037.79$

0.42

5.13

$6,808.40$

$29,053.84$

0.68

0.42

$8,253.48$

43.67

$37,807.79$

66.54

61.10

994.08

193.25

$4,725.05$

$86,641.44$

$21,372.83$

$1,183.80$

$1,368.53$

919.62

0.07

377.88

0.73

0.08

$3,717.50$

0.88

$12,701.84$

$3,650.70$

0.02

$4,315.70$

3.05

a. Minnesota totals include $42.20 \mathrm{ft}^{3}$ and 1.19 curies generated in Hong Kong by a U.S. owned, Minnesota based, Company. 
Table 1. (continued)

\begin{tabular}{|c|c|c|}
\hline States & $\begin{array}{c}\text { Volume } \\
\left(\mathrm{ft}^{3}\right)\end{array}$ & $\begin{array}{l}\text { Activity } \\
\text { (curies) }\end{array}$ \\
\hline Oregon & $59,456.32$ & 592.90 \\
\hline Pennsylvania & $101,604.10$ & $47,305.67$ \\
\hline Rhode Island & 159.83 & 0.25 \\
\hline South Carolina & $46,927.41$ & $2,208.45$ \\
\hline South Dakota & $1,169.64$ & 0.05 \\
\hline Tennessee & $83,401.85$ & $1,209.64$ \\
\hline Texas & $9,201.67$ & $1,650.32$ \\
\hline Utah & $5,338.12$ & 1.40 \\
\hline Virginia & $57,765.63$ & $1,923.95$ \\
\hline Washington & $26,141.09$ & $1,397.77$ \\
\hline West Virginia & 95.18 & 0.19 \\
\hline Wisconsin & $9,217.32$ & 569.68 \\
\hline Wyoming & 15.00 & 1.88 \\
\hline Total & $1,142,809.92$ & $547,901.89$ \\
\hline
\end{tabular}




\section{1990 REPORTING METHOD AND SUMMARY}

The data for the 1990 State-by-State report were developed from the Department of Energy's Manifest Information System with data originating from the LLW shipment manifests recorded at the three commercial LLW disposal sites located at Barnwell, South Carolina; Beatty, Nevada; and Richland, Washington. ${ }^{\mathrm{b}}$ This information is presented in Appendix A.

Reports in this series generated for 1979 through 1987 have attributed waste processed or consolidated into larger shipments at intermediate waste management facilities to the states in which those facilities were located. The reports generated for 1988 and subsequent years attribute waste volume, activity, and generator type by source to the original state in which it was generated, even when brokered shipments included waste generated in more than one state. This reporting change was made possible because of modifications in the manifesting requirements for brokered shipments at the sited states. The waste volumes for states such as Tennessee, where a significant volume of waste is processed at several large facilities, were the most notably affected.

Two classifications of waste shipments are identified in this year's report.

- Non-Direct: Shipments from the generator sent to the disposal facility through an intermediary

- Direct: Shipments sent directly to the disposal facility.

In 1990 approximately $36 \%$ of all waste shipped for disposal was categorized as Non-Direct.

The data are arranged to help the reader locate information, ranging from the total commercial LLW disposed of within the United States to the specifics of LLW disposed of by each state.

The information presented covers the distribution of LLW by:

- Volume

- Activity

- Waste classification

- Waste generator type

- Disposal site

- Waste that has been subject to intermediate handling.

b. Data obtained from Chem-Nuclear Systems, Inc., for the Barnwell, South Carolina, low-level radioactive waste disposal site, as defined by subcontract number $\mathrm{C} 87-1.31476$, and data obtained from U.S. Ecology, Inc. operated low-level radioactive waste disposal sites located in Beatty, Nevada, and Richland, Washington. from Utility Data Institute, as defined by subcontract number C87-131477. Utility Data Institute is the exclusive licensee and marketing agent for the American Ecology Time Sharing Service. 
Waste generator types include:

- Academic

- Government

- Industrial.

- Medical

- Utility

The utility category includes commercial nuclear power reactors. The academic category includes university hospitals and university medical and nonmedical research facilities. Medical generators include hospitals and clinics, research facilities, and private medical offices. The industrial category includes private entities such as research and development companies, manufacturers, nondestructive testing, mining, fuel isbrication facilities, and radiopharmaceutical manufacturers. The government category includes state and Federal agencies.

Appendix B contains a comparison of LLW disposal volumes by state for the most recent five-year period, and a complete list of the commercial nuclear power reactors located in the United States as of December 31, 1990.

A review of the data found in this report will show that, in 1990, commercial LLW disposal facilities received a total volume of $1,142,810$ cubic feet of waste, with an activity of 547,902 curies. Waste distribution by disposal site is presented in Table 2; Tables 3 and 4 provide typical radionuclide and waste forms associated with commercial LLW. 
Table 2. Distribution of low-level radioactive waste received at disposal sites in 1990)

\begin{tabular}{|c|c|c|c|c|}
\hline Site & $\begin{array}{c}\text { Volume } \\
\left(\mathrm{ft}^{3}\right)\end{array}$ & $\begin{array}{c}\text { Percent } \\
\text { of } \\
\text { Total } \\
\end{array}$ & $\begin{array}{l}\text { Activity } \\
\text { (curies) }\end{array}$ & $\begin{array}{c}\text { Percent } \\
\text { of } \\
\text { Total }\end{array}$ \\
\hline Barnwell & 788,032 & 69 & 443,594 & 81 \\
\hline Beatty & 59,479 & 5 & 11,323 & 2 \\
\hline Richland & 295,299 & 26 & 92,985 & 17 \\
\hline Total & $1,142,810$ & 100 & 547,902 & 100 \\
\hline
\end{tabular}

Table 3. Most frequently reported low-level radioactive waste radionuclides received at disposal sites in 1990

\begin{tabular}{|c|c|c|c|c|}
\hline Reactors & Academic & Medical & Industrial & Government \\
\hline${ }^{60} \mathrm{Co}$ & ${ }^{125}{ }_{I}$ & ${ }^{51} \mathrm{Cr}$ & NatTh & ${ }^{60} \mathrm{Co}$ \\
\hline${ }^{14} \mathrm{C}$ & ${ }^{3} \mathrm{H}$ & ${ }^{3} \mathrm{H}$ & ${ }^{\mathrm{Nat}} \mathrm{U}$ & ${ }^{55} \mathrm{Fe}$ \\
\hline${ }^{55} \mathrm{Fe}$ & ${ }^{35} \mathrm{~S}$ & ${ }^{125} I$ & $238 \mathrm{U}$ & ${ }^{54} \mathrm{Mn}$ \\
\hline${ }^{3} \mathrm{H}$ & ${ }^{14} \mathrm{C}$ & ${ }^{35} \mathrm{~S}$ & ${ }^{14} \mathrm{C}$ & ${ }^{63} \mathrm{Ni}$ \\
\hline${ }^{137} \mathrm{Cs}$ & ${ }^{51} \mathrm{Cr}$ & ${ }^{14} \mathrm{C}$ & ${ }^{3} \mathrm{H}$ & ${ }^{58} \mathrm{Co}$ \\
\hline${ }^{54} \mathrm{Mn}$ & ${ }^{32} \mathrm{P}$ & ${ }^{57} \mathrm{Co}$ & ${ }^{137} \mathrm{Cs}$ & ${ }^{14} \mathrm{C}$ \\
\hline${ }^{58} \mathrm{Co}$ & ${ }^{45} \mathrm{Ca}$ & ${ }^{59} \mathrm{Fe}$ & ${ }^{60} \mathrm{Co}$ & \\
\hline${ }^{99} \mathrm{Tc}$ & & ${ }^{32} \mathrm{P}$ & ${ }^{241} \mathrm{Pu}$ & \\
\hline${ }^{63} \mathrm{Ni}$ & & ${ }^{238} \mathrm{U}$ & ${ }^{226} \mathrm{Ra}$ & \\
\hline${ }^{129} I$ & & & & \\
\hline
\end{tabular}


Table 4. Typical waste forms, by generator categories ${ }^{5}$

\section{Academic}

Compacted trash or solids

Institutional laboratory or biological waste

Absorbed liquids

Animal carcasses

\section{Government}

Compacted trash or solids

Contaminated plant hardware

Absorbed liquids

\section{Industrial}

Depleted uranium

Compacted trash or solids

Contaminated plant hardware

Absorbed liquids

Sealed sources

\section{Medical}

Compacted trash or solids

Institutional laboratory or biological waste

Absorbed liquids

Sealed sources

\section{Utilities}

Spent resins

Evaporator bottoms and concentrated waste

Filter sludges

Dry compressible waste

Irradiated components

Contr minated plant hardware 


\section{REFERENCES}

1. U.S. Nuclear Regulatory Commission, Measuring, Evaluating, and Reporting Radioactivity in Solid Wastes and Releases of Radioactive Materials in Liquid and Gaseous Effluents from Light Water Cooled Nuclear Power Plants, Regulatory Guide 1.21. Revision 1, June 1974.

2. U.S. Department of Energy, Solid Waste Information Management System (SWIMS) - Actual Solid Waste Generated for Fiscal Year 1979, February 22, 1980.

3. R. L. Anderson, L. R. Cooley, T. J. Beck, C. S. Strauss, Institutional Radioactive Waste, NUREG/ CR-O028, Radiation Safety Office, University of Maryland at Baltimore, March 1978.

4. T. J. Beck, L. R. Cooley, M. R. McCampbell, Institutional Radioactive Wastes-1977. Radiation Safety Office, University of Maryland at Baltimore (draft), June 1979.

5. Conference of Radiation Control Program Directors, Inc., "Low-Level Radioactive Waste Management Survey Summary, " Frankfort, Kentucky, 1984. 
I

I

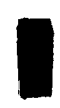

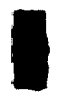

I

I

I

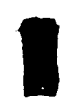

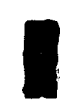

I

1

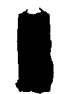

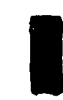

I

I

I

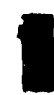

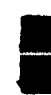

1 


\section{APPENDIX A}

\section{LOW-LEVEL RADIOACTIVE WASTE ASSESSMENTS BY COMPACT REGION AND STATE}




\section{INDEX OF STATES}

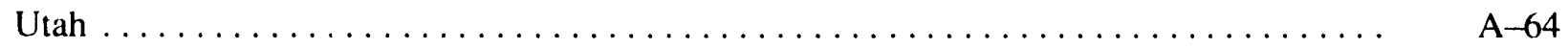

Vermont

2 (Sec. 1)

Virginia

A-94

Washington

A-66

West Virginia

A-10

Wisconsin

A -44

Wyoming

A-76 
I

I

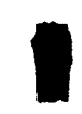

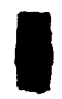

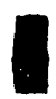

I

,

,

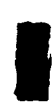

,

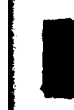

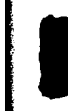

,

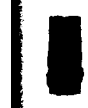

I

I

,

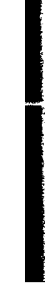




\section{UNITED STATES \\ Low-Level Radioactive Wastes Received at Commercial Disposal Sites ${ }^{a}$}

\begin{tabular}{lrr}
$\begin{array}{l}\text { Generator } \\
\text { Category }\end{array}$ & \multicolumn{1}{c}{$\begin{array}{c}\text { Volume } \\
\left(\mathrm{ft}^{3}\right)\end{array}$} & \multicolumn{1}{c}{$\begin{array}{c}\text { Activity } \\
\text { (curies) }\end{array}$} \\
Academic & $48,555.10$ & $1,096.04$ \\
Government & $72,353.51$ & $10,151.86$ \\
Industrial & $356,343.98$ & $102,855.67$ \\
Medical & $22,792.13$ & 59.45 \\
Utility & $642,765.20$ & $433,738.88$ \\
Total & $1,142,809.92$ & $547,901.89$
\end{tabular}

VOLUME PERCENTAGE BY SOURCE

(Percentages <. $1 \%$ are not displayed)

\section{ACTIVITY PERCENTAGE BY SOURCE}

(Percentages <. $1 \%$ are not displayed)
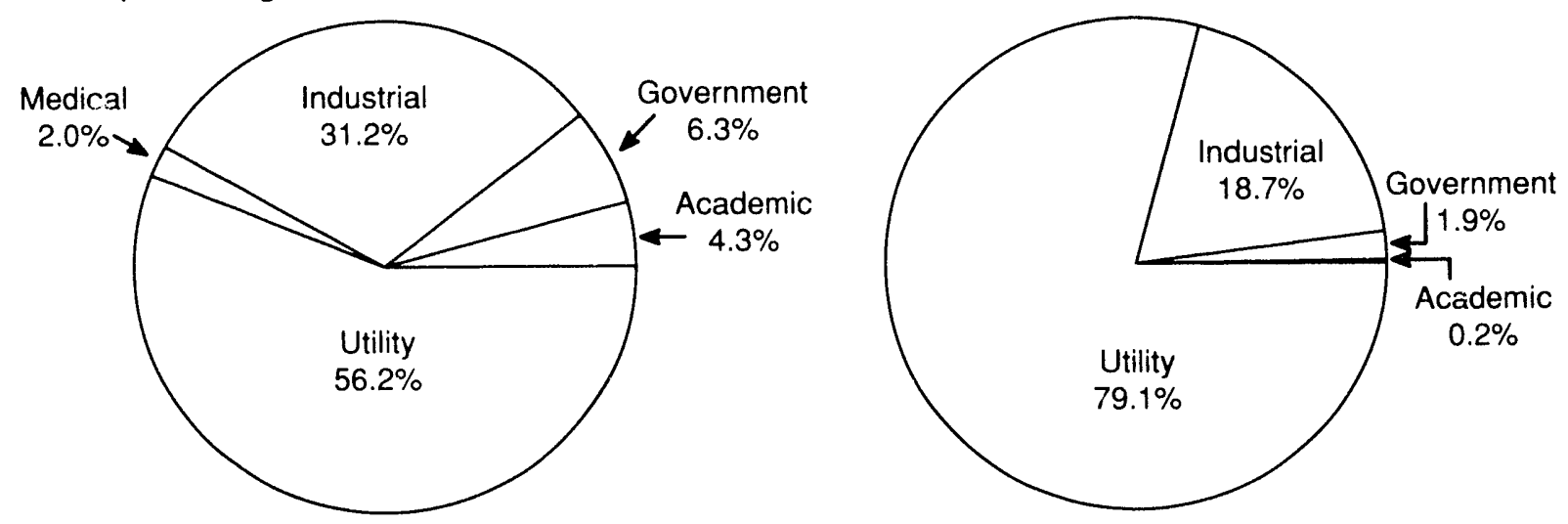

a. Data in this, and all succeeding pages in Appendix A, were provided by the Department of Energy's Manifest Information System. Data originated with Chem Nuclear Systems, Inc., and Utility Data Inst., Inc., from References (5) and (6) of the main text. 


\section{UNITED STATES \\ Low-Level Radioactive Wastes Received \\ at Commercial Disposal Sites}

Disposal
Site

Barnwell

Beatty

Richland

Total

$$
\begin{gathered}
\text { Volume } \\
\left(\mathrm{ft}^{3}\right)
\end{gathered}
$$

$788,031.90$

$59,479.22$

$295,298.80$

$1,142,809.92$
Activity

(curies)

$443,594.19$

$11,322.77$

$92,984.93$

$547,901.89$
VOLUME PERCENTAGE BY DISPOSAL SITE

(Percentages <. $1 \%$ are not displayed)

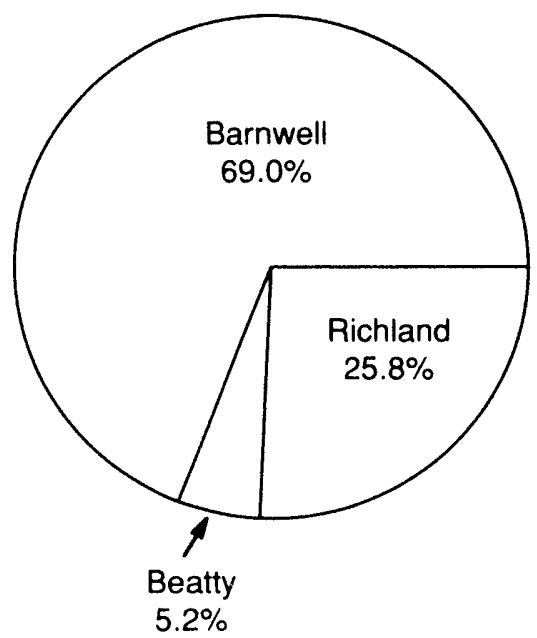

ACTIVITY PERCENTAGE BY DISPOSAL SITE

(Percentages <. $1 \%$ are not displayed)

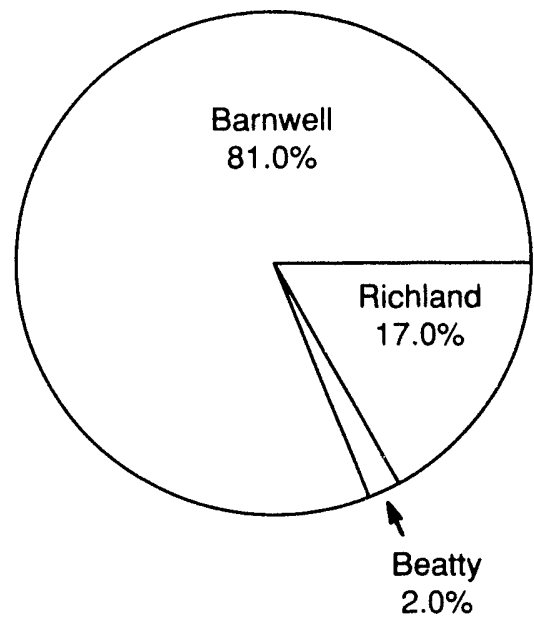

Volume Percentage by Waste Class for Direct Shipments

(Percentages <. $1 \%$ are not displayed)
Direct Shipment

Waste Class

A

B

C

Total

Grand Total (curies)

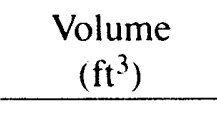

$1,106,774.95$

$26,531.09$

$9,518.88$

$1,142.809 .92$

$547,901.89$

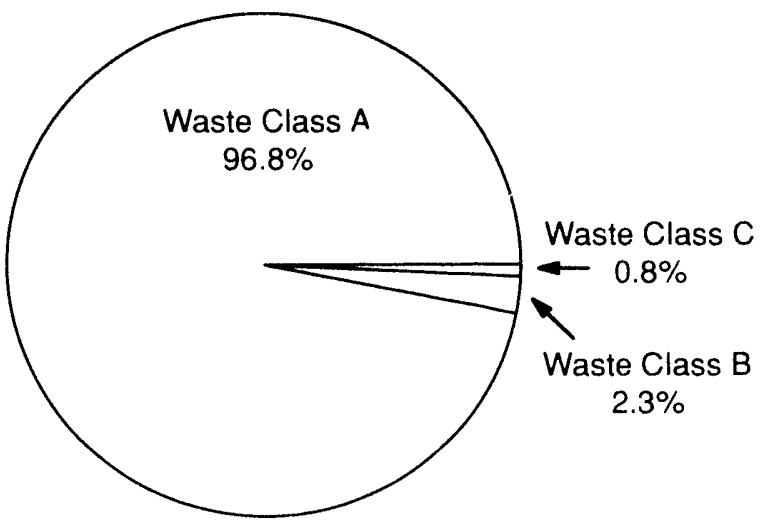





\section{APPALACHIAN COMPACT Low-Level Radioactive Wastes Received at Commercial Disposal Sites}

\begin{tabular}{lrr}
\multicolumn{1}{c}{ States } & \multicolumn{1}{c}{$\begin{array}{c}\text { Volume } \\
\left(\mathrm{ft}^{3}\right)\end{array}$} & \multicolumn{1}{c}{$\begin{array}{c}\text { Activity } \\
\text { (curies) }\end{array}$} \\
Delaware & 843.83 & 0.42 \\
Maryland & $17,037.94$ & $4,725.05$ \\
Pennsylvania & $101,604.10$ & $47,305.67$ \\
West Virginia & 95.18 & 0.19 \\
Total & $119,581.05$ & $52,031.33$
\end{tabular}

VOLUME PERCENTAGE BY STATE

(Percentages <. $1 \%$ are not displayed)

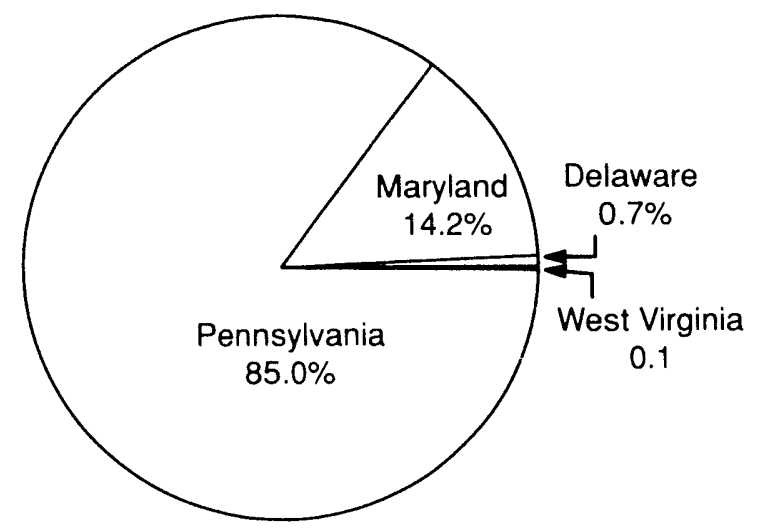

VOLUME PERCENTAGE BY CATEGORY

(Percentages $<.1 \%$ are not displayed)

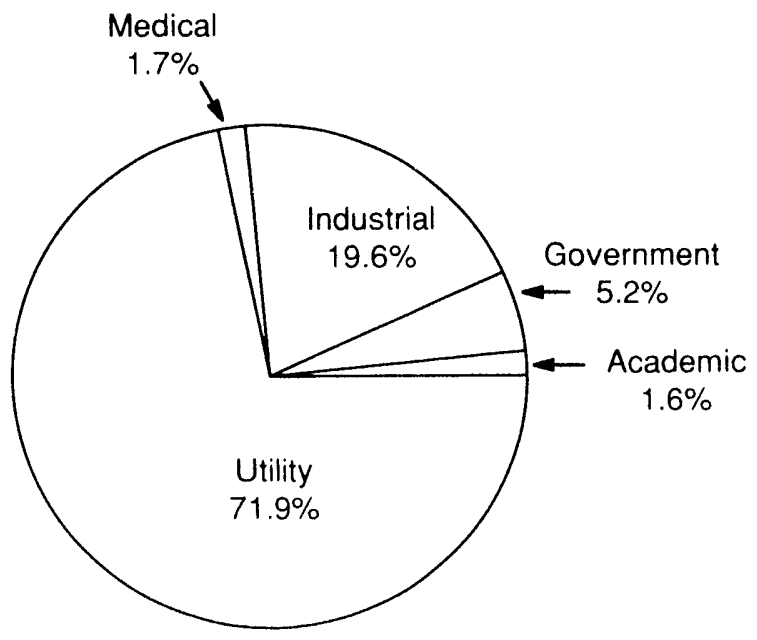




\section{DELAWARE \\ Low-Level Radioactive Wastes Received at Commercial Disposal Sites}

\section{Generator \\ Category}

Academic

Industrial

Total
Volume

$\left(\mathrm{ft}^{3}\right)$

47.93

795.90

843.83
Activity (curies)

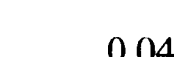

0.38

0.42

VOLUME PERCENTAGE BY SOURCE

(Percentages <. $1 \%$ are not displayed)

ACTIVITY PERCENTAGE BY SOURCE

(Percentages <. $1 \%$ are not displayed)
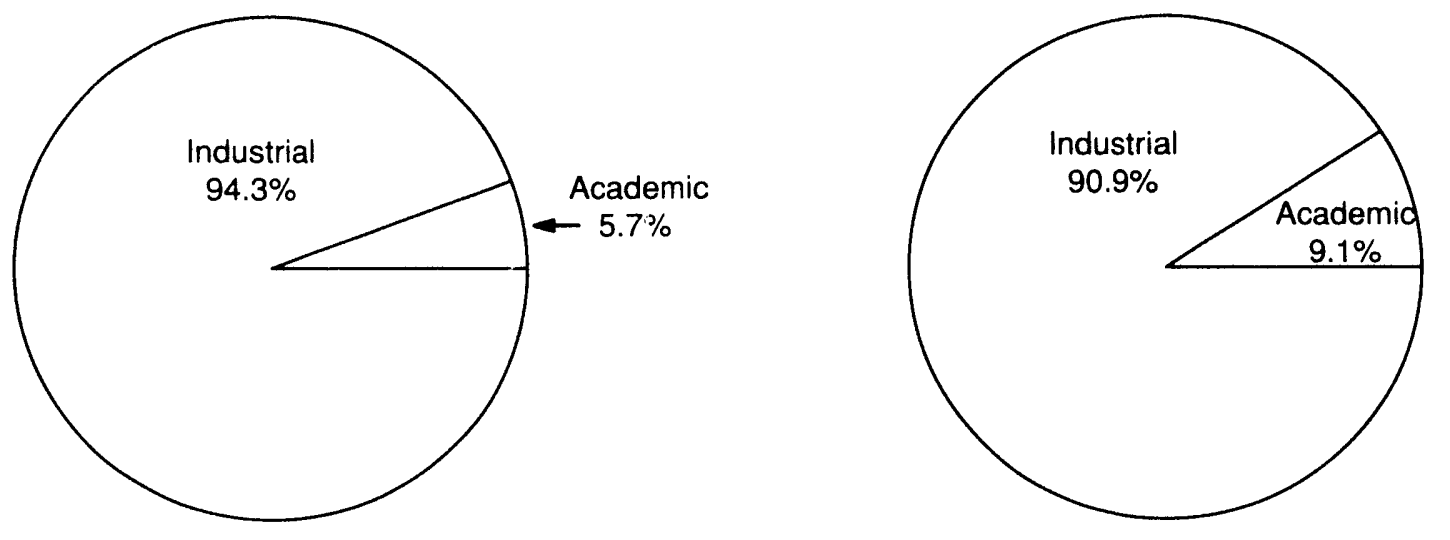


\section{DELAWARE \\ Low-Level Radioactive Wastes Received at Commercial Disposal Sites}

\begin{tabular}{c} 
Disposal \\
Site \\
\hline
\end{tabular}

Barnwell

Richland

Total

\author{
Volume \\ $\left(\mathrm{ft}^{3}\right)$
}

130.89

712.94

843.83
Activity

(curies)

0.11

0.31

0.42
VOLUME PERCENTAGE BY DISPOSAL SITE

(Percentages <. $1 \%$ are not displayed)

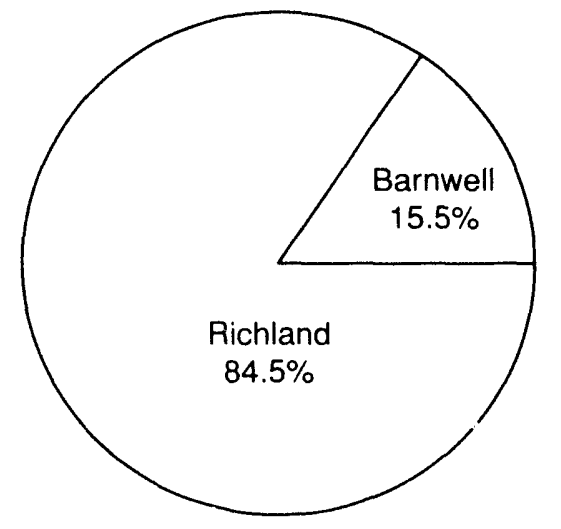

ACTIVITY PERCENTAGE BY DISPOSAL SITE

(Percentages $<.1 \%$ are not displayed)

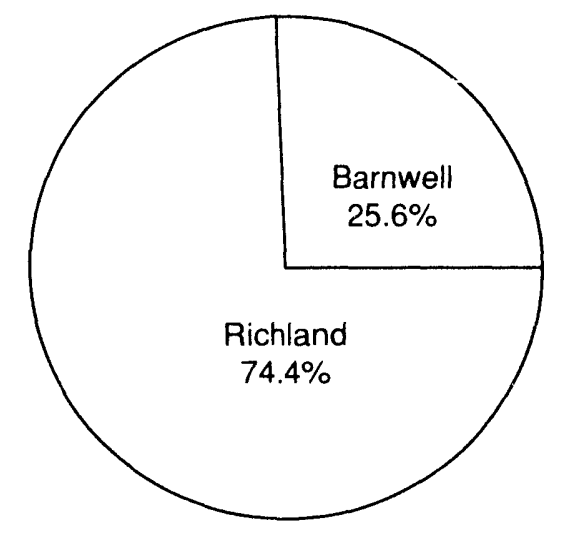

Volume Percentage by Waste Class

for Direct Shipments and Total

Non-Direct Volume Percentage

(Percentages $<.1 \%$ are not displayed)

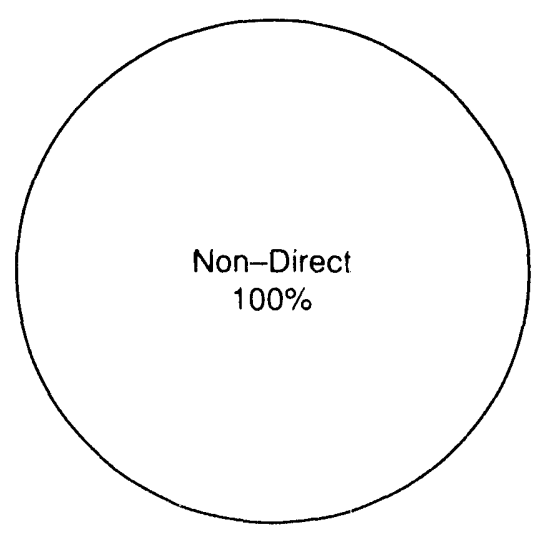




\section{MARYLAND \\ Low-Level Radioactive Wastes Received at Commercial Disposal Sites}

\section{Generator \\ Category}

Academic

Government

Industrial

Medical

Utility

Total

$\begin{array}{r}\multicolumn{1}{c}{\begin{array}{c}\text { Volume } \\ \left(\mathrm{ft}^{3}\right)\end{array}} \\ \hline 417.58 \\ 5,733.89 \\ 6,045.82 \\ 302.95 \\ 4,537.70 \\ \hline\end{array}$

$17,037.94$
Activity

(curies)

1.45

82.10

524.06

0.37

$4,117.08$

$4,725.05$
VOLUME PERCENTAGE BY SOURCE (Percentages <. $1 \%$ are not displayed)

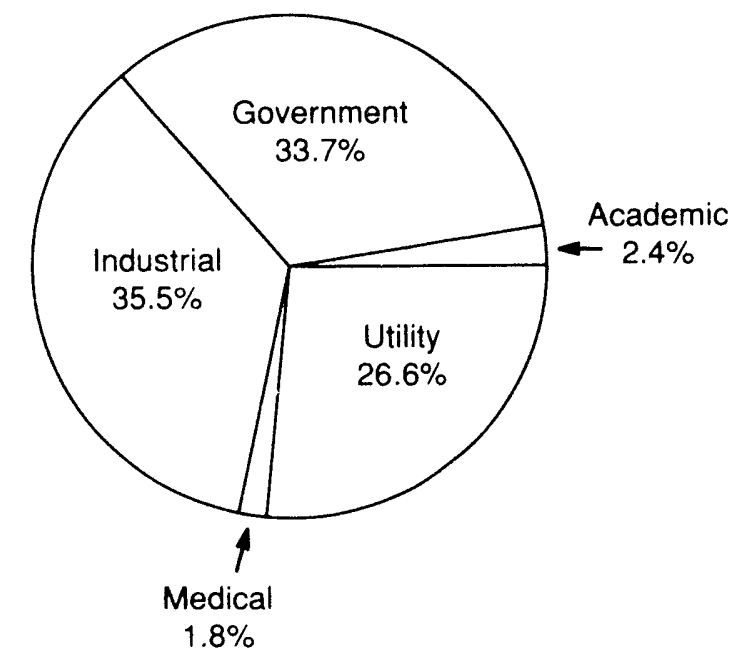

ACTIVITY PERCENTAGE BY SOURCE (Percentages <. $1 \%$ are not displayed)

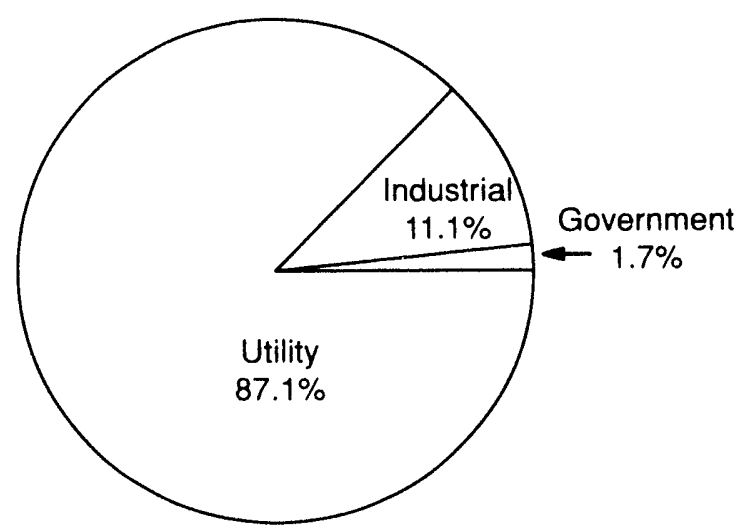




\section{MARYLAND \\ Low-Level Radioactive Wastes Received at Commercial Disposal Sites}

\begin{tabular}{c} 
Disposal \\
Site \\
\hline
\end{tabular}

Barnwell

Beatty

Richland

Total

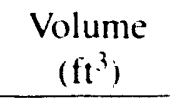

15.412 .88

586.39

1.038 .67

17.037 .94
Activity

(curies)

4.691 .89

31.18

1.97

$4,725.05$
VOLUME PERCENTAGE BY DISPOSAL SITE (Percentages <. $1 \%$ are not displayed)

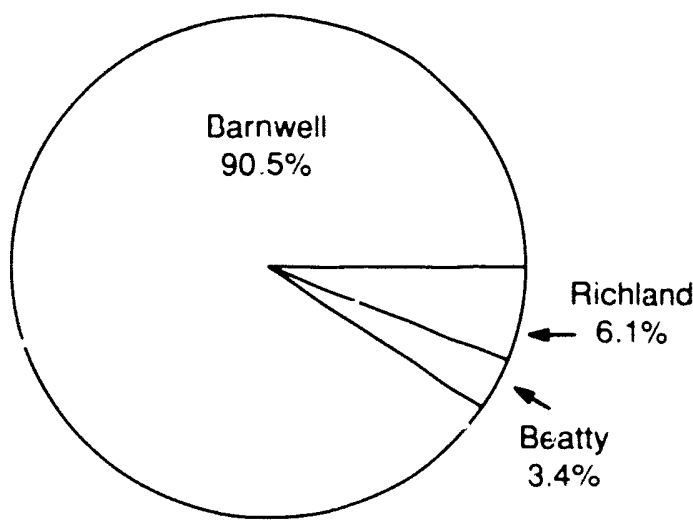

Direct Shipment

Waste Class

A

B

C

Total

Non-Direct Shipments

Grand Total

Total Direct Shipme, it

Activity (curies)

Total Non-Direct Shipment

Activity (curies)

Grand Total (curies)
Volume

$\left(\mathrm{ft}^{3}\right)$

6.049 .68

24() .60

172.30

6.462 .58

10.575 .36

17.0.37.94

4.603 .37

121.68

4.725 .05
ACTIVITY PERCENTAGE BY DISPOSAL SITE

(Percentages <. $1 \%$ are not displayed)

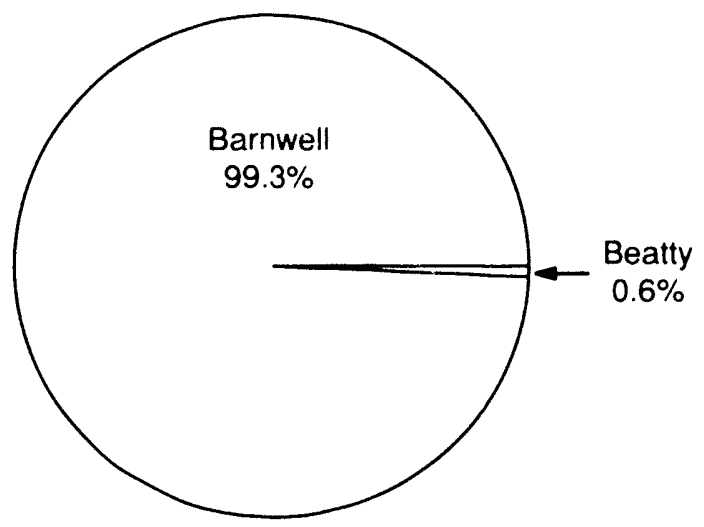

Volume Percentage trv Waste Class for Direct Shipmenis and Total Non-Direct Volume Perceiltage (Percentages <. $1 \%$ are not displayed)

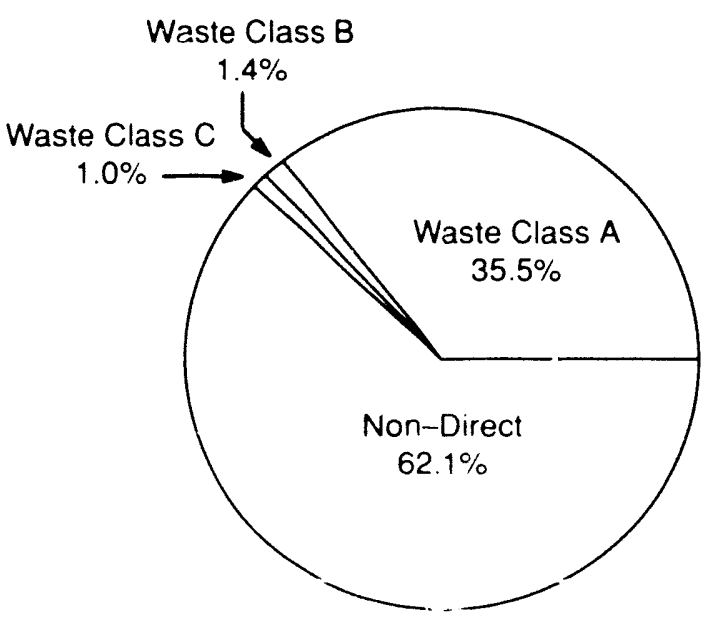




\section{PENNSYLVANIA \\ Low-Level Radioactive Wastes Received at Commercial Disposal Sites}

\author{
Generator \\ Category \\ Academic \\ Government \\ Industrial \\ Medical \\ Utility
}

Total

$$
\begin{array}{r}
\begin{array}{c}
\text { Volume } \\
\left(\mathrm{ft}^{3}\right)
\end{array} \\
\hline 1,501.76 \\
490.36 \\
16.573 .75 \\
1,655.25 \\
81,382.98 \\
\hline
\end{array}
$$

$101,604.10$
Activity (curies)

$$
2.59
$$

$3,688.80$

166.26

2.74

$43,445.28$

$47,305.67$

VOLUME PERCENTAGE BY SOURCE (Percentages <. $1 \%$ are not displayed)
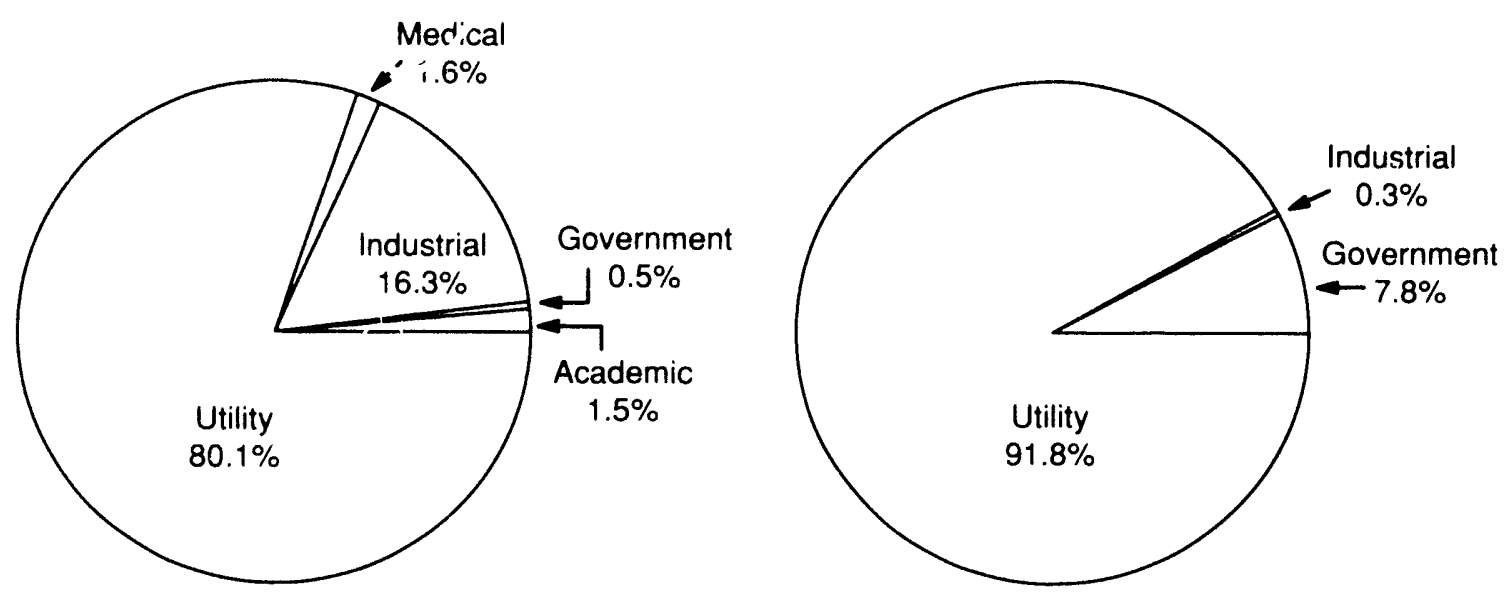


\section{PENNSYLVANIA \\ Low-Level Radioactive Wastes Received at Commercial Disposal Sites}

\begin{tabular}{lrr}
\multicolumn{1}{c}{$\begin{array}{c}\text { Disposal } \\
\text { Site }\end{array}$} & \multicolumn{1}{c}{$\begin{array}{c}\text { Volume } \\
\left(\mathrm{ft}^{3}\right)\end{array}$} & $\begin{array}{r}\text { Activity } \\
\text { (curies) }\end{array}$ \\
Barnwell & $84,221.23$ & $46,141.14$ \\
Beatty & $1,963.21$ & 795.61 \\
Richland & $15,419.66$ & 368.92 \\
\hline & $101,604.10$ & 47.305 .67
\end{tabular}

\section{VOLUME PERCENTAGE BY DISPOSAL SITE \\ (Percentages $<.1 \%$ are not displayed)}

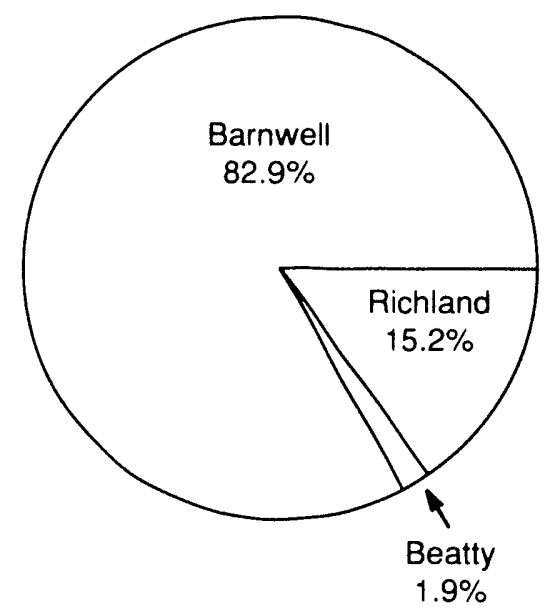

ACTIVITY PERCENTAGE BY DISPOSAL SITE (Percentages <. $1 \%$ are not displayed)

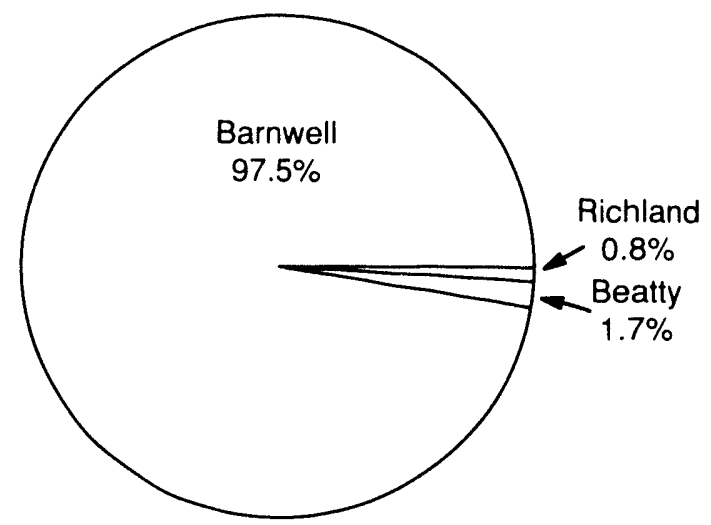

Volume Percentage by Waste Class for Direct Shipments and Total Non-Direct Volume Percentage (Percentages <.1\% are not displayed)

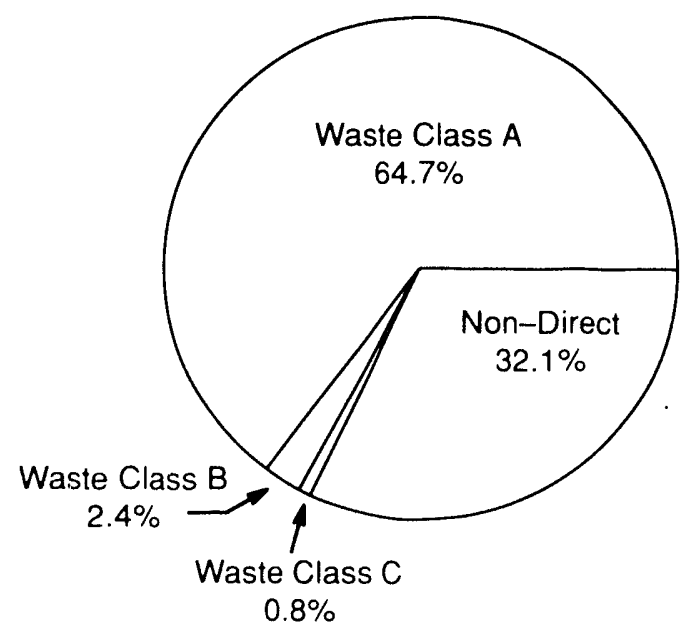

Direct Shipment

Waste Class

$$
\begin{aligned}
& \text { A } \\
& \text { B } \\
& \text { C }
\end{aligned}
$$

Total

Non-Direct Shipments

Grand Total

Total Direct Shipment

Activity (curies)

Total Non-Direct Shipment

Activity (curies)

Grand Tota! (curies)
Volume

$\left(\mathrm{ft}^{3}\right)$

$65,719.30$

$2,451.20$

773.30

$68,943.80$

$32,66() .30$

$101,6(04.10$

$42,700.31$

4.605 .36

47.305 .67 


\section{WEST VIRGINIA \\ Low-Level Radioactive Wastes Received at Commercial Disposal Sites}

\author{
Generator \\ Category \\ Government \\ Industrial \\ Medical
}

Total

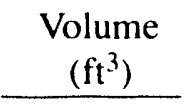

2.00

15.68

77.50

95.18
Activity

(curies)

$<.01$

0.06

0.13

0.19

VOLUME PERCENTAGE BY SOURCE

ACTIVITY PERCENTAGE BY SOURCE

(Percentages <. $1 \%$ are not displayed)

(Percentages <. $1 \%$ are not displayed)
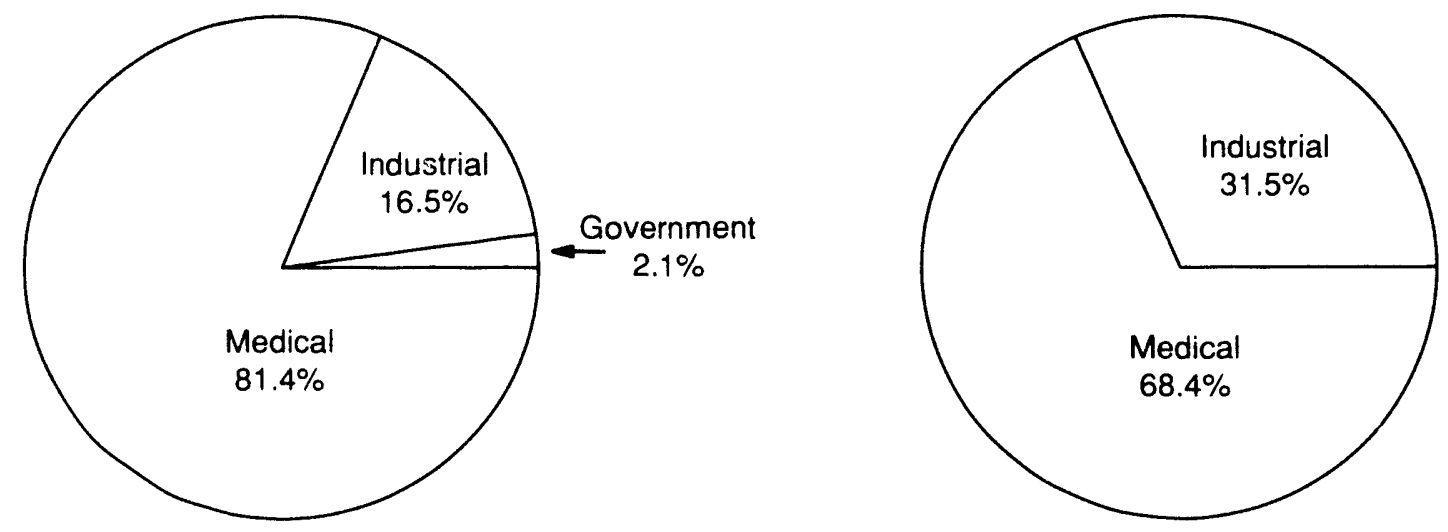


\section{WEST VIRGINIA \\ Low-Level Radioactive Wastes Received at Commercial Disposal Sites}

\begin{tabular}{lrr}
\multicolumn{1}{c}{$\begin{array}{c}\text { Disposal } \\
\text { Site }\end{array}$} & $\begin{array}{c}\text { Volume } \\
\left(\mathrm{ft}^{3}{ }^{3}\right)\end{array}$ & $\begin{array}{r}\text { Activity } \\
\text { (curies) }\end{array}$ \\
\cline { 2 - 3 } Barnwell & 0.68 & $<.01$ \\
Beatty & 19.50 & 0.06 \\
Richland & 75.00 & 0.13 \\
Total & 95.18 & 0.19
\end{tabular}

VOLUME PERCENTAGE BY DISPOSAL SITE

(Percentages <. $1 \%$ are not displayed)

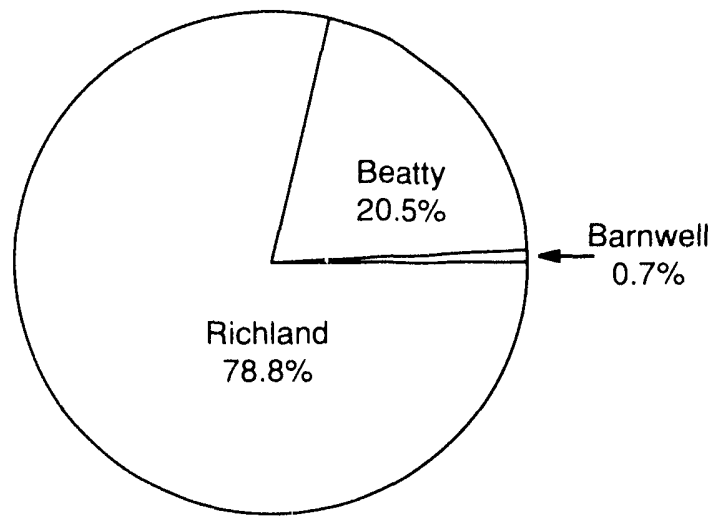

ACTIVITY PERCENTAGE BY DISPOSAL SITE

(Percentages $<.1 \%$ are not displayed)
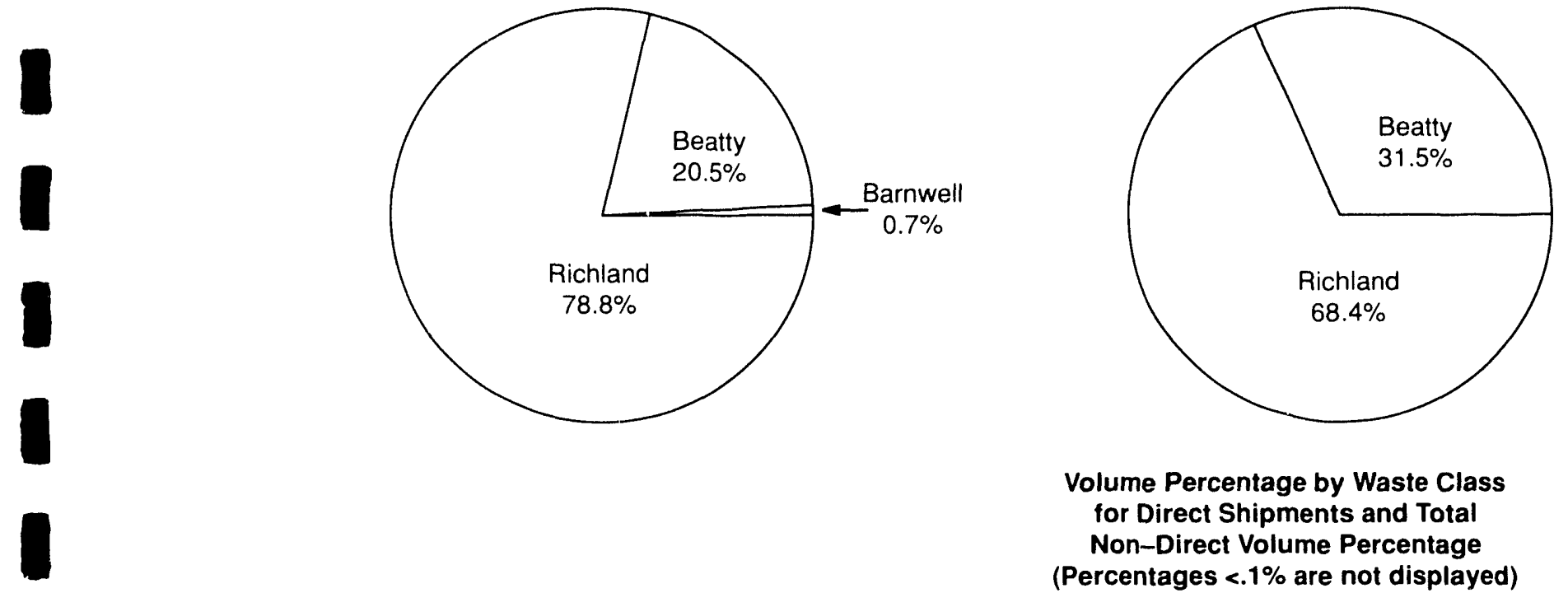

Volume Percentage by Waste Class

for Direct Shipments and Total

Non-Direct Volume Percentage

(Percentages $<.1 \%$ are not displayed)

Direct Shipment

Waste Class

A

B

C

Total

Non-Direct Shipments

Grand Total

Total Direct Shipment

Activity (curies)

Total Non-Direct Shipment

Activity (curies)

Grand Total (curies)
Volume

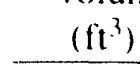

0.68

0.00

0.00

0.68

94.50)

95.18

$<$.01

0.19

0.19

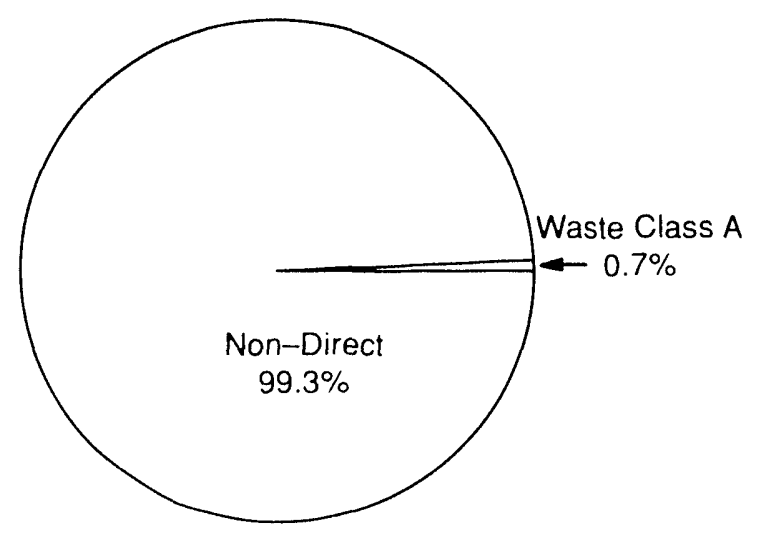




$$
\begin{aligned}
& 1 \\
& 1 \\
& 1 \\
& 1 \\
& 1 \\
& 1 \\
& 1 \\
& 1 \\
& 1 \\
& 1 \\
& 1 \\
& 1 \\
& 1 \\
& 1 \\
& 1 \\
& 1 \\
& 1 \\
& 1 \\
& 1
\end{aligned}
$$




\section{CENTRAL COMPACT \\ Low-Level Radioactive Wastes Received at Commercial Disposal Sites}

\begin{tabular}{lrr}
\multicolumn{1}{c}{ States } & \multicolumn{1}{c}{$\begin{array}{c}\text { Volume } \\
\left(\mathrm{ft}^{3}{ }^{3}\right)\end{array}$} & \multicolumn{1}{c}{$\begin{array}{c}\text { Activity } \\
\text { (curies) }\end{array}$} \\
Arkansas & $6,239.42$ & 14.39 \\
Kansas & $3,232.54$ & 66.54 \\
Louisiana & $11,329.30$ & 994.08 \\
Nebraska & $15,558.87$ & 377.88 \\
Oklahoma & $21,967.85$ & 3.05 \\
Total & $58,327.98$ & $1,455.94$
\end{tabular}

VOLUME PERCENTAGE BY STATE

(Percentages <. $1 \%$ are not displayed)

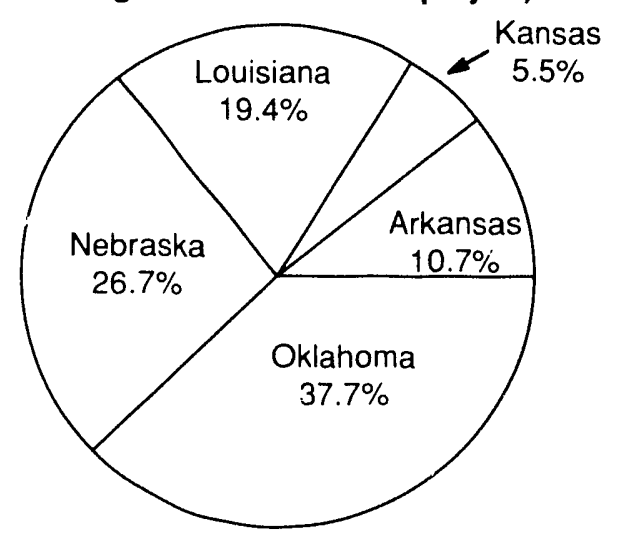

VOLUME PERCENTAGE BY CATEGORY

(Percentages $<.1 \%$ are not displayed)

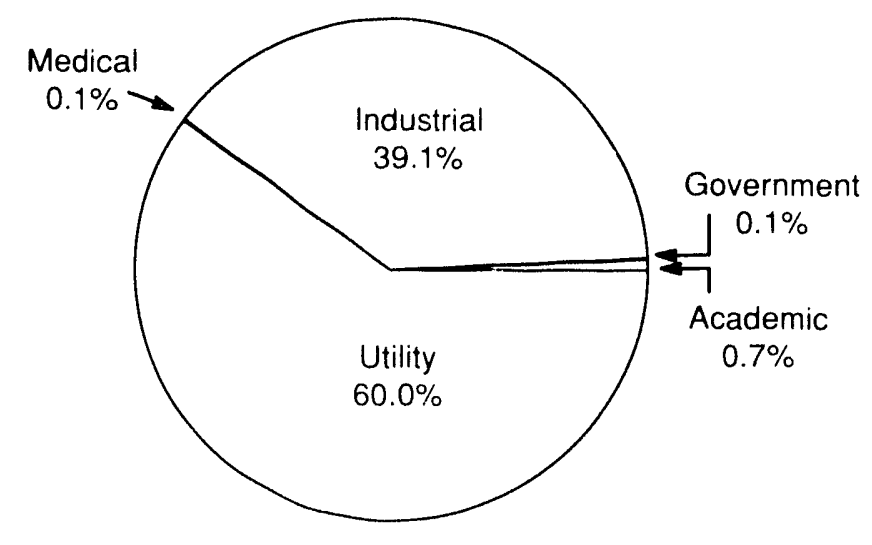




\section{ARKANSAS \\ Low-Level Radioactive Wastes Received at Commercial Disposal Sites}

\begin{tabular}{l} 
Generator \\
Category \\
\hline
\end{tabular}

Academic

Industrial

Medical

Utility

Total

$\begin{array}{r}\begin{array}{c}\text { Volume } \\ \left(\mathrm{ft}^{3}\right)\end{array} \\ \hline 200.96 \\ 7.50 \\ 43.94 \\ 5,987.02 \\ \hline\end{array}$

$6,239.42$
Activity

(curies)

0.03

$<.01$

0.05

14.30

14.39
VOLUME PERCENTAGE BY SOURCE

(Percentage $<.1 \%$ are not displayed)

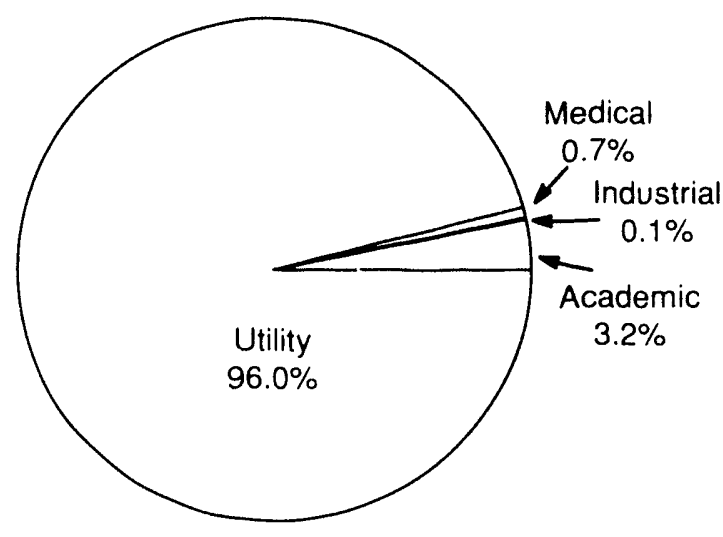

ACTIVITY PERCENTAGE BY SOURCE

(Percentages $<.1 \%$ are not displayed)

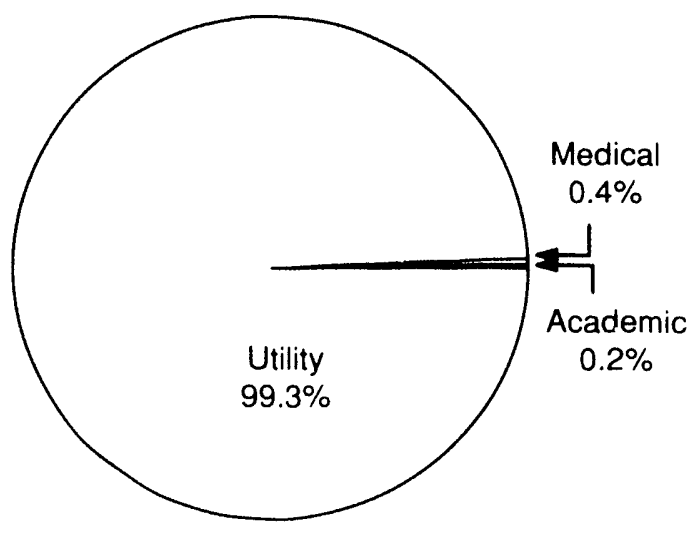




\title{
ARKANSAS \\ Low-Level Radioactive Wastes Received at Commercial Disposal Sites
}

\begin{tabular}{c} 
Disposal \\
Site \\
\hline
\end{tabular}

Barnwell

Beatty

Richland

Total
Volume
$\left(\mathrm{ft} \mathrm{t}^{3}\right)$

5.987 .02

2.54

249.86

$6,239.42$
Activity

(curies)

$\begin{array}{r}14.30 \\ 0.05 \\ 0.03 \\ \hline\end{array}$

14.39

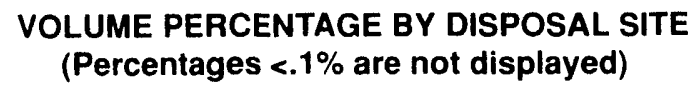

(Percentages <. $1 \%$ are not displayed)

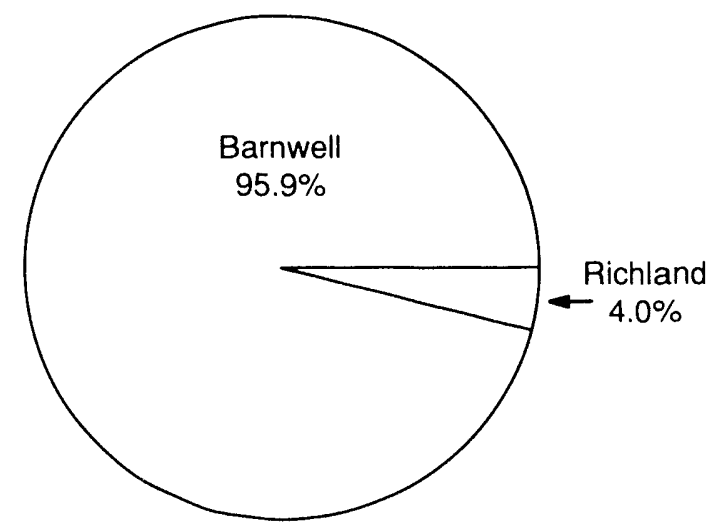

ACTIVITY PERCENTAGE BY DISPOSAL SITE

(Percentages $>.1 \%$ Are Not Displayed)

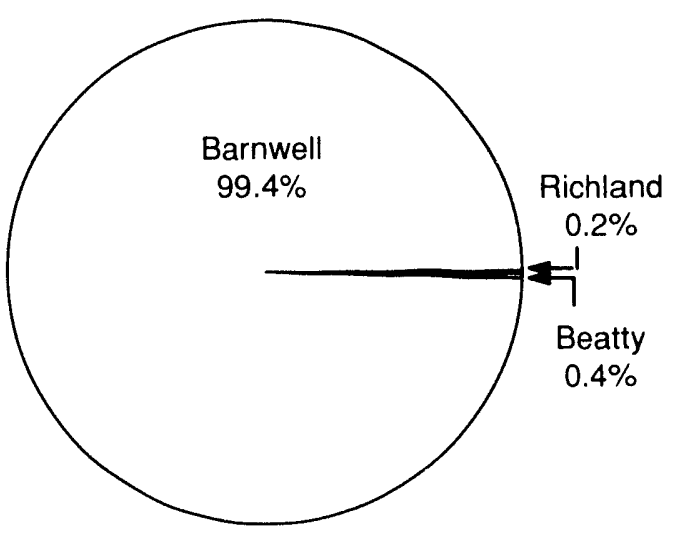

\begin{abstract}
Volume Percentage by Waste Class for Direct Shipments and Total

Non-Direct Volume Percentage

(Percentages <. $1 \%$ are not displayed)
\end{abstract}

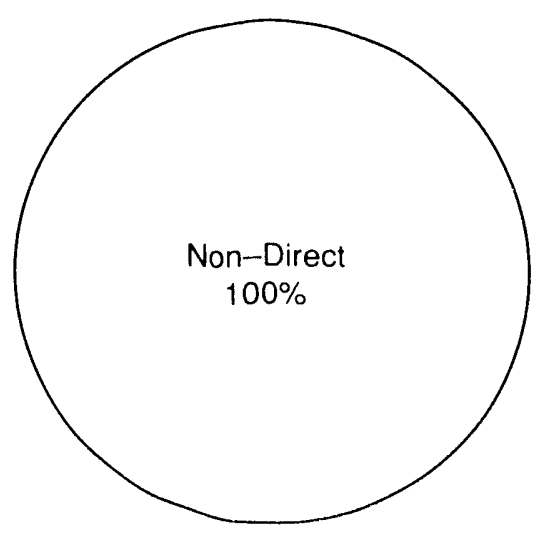




\section{KANSAS \\ Low-Level Radioactive Wastes Received at Commercial Disposal Sites}

\begin{tabular}{lrr}
$\begin{array}{l}\text { Generator } \\
\text { Category }\end{array}$ & $\begin{array}{c}\text { Volume } \\
\left(\mathrm{ft}^{3}\right)\end{array}$ & $\begin{array}{r}\text { Activity } \\
(\text { curies })\end{array}$ \\
\cline { 2 - 2 } & 39.79 & 0.13 \\
Academic & 7.50 & $<.01$ \\
Government & 248.88 & 34.30 \\
Industrial & 0.27 & 0.39 \\
Medical & 2.936 .10 & 31.73 \\
Utility & $3,232.54$ & 66.54
\end{tabular}

VOLUME PERCENTAGE BY SOURCE

(Percentage $<.1 \%$ are not displayed)

ACTIVITY PERCENTAGE BY SOURCE

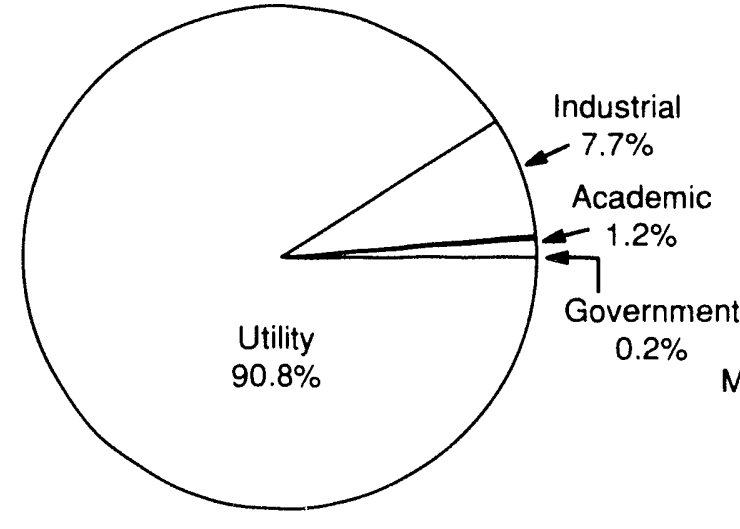

(Percentages <. $1 \%$ are not displayed)

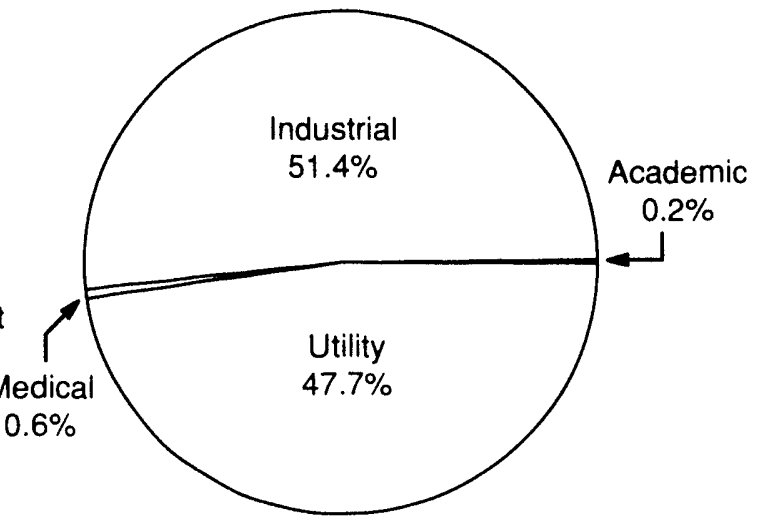




\section{KANSAS \\ Low-Level Radioactive Wastes Received at Commercial Disposal Sites}

\begin{tabular}{c} 
Disposal \\
Site \\
\hline
\end{tabular}

Barnwell

Beatty

Richland

Total

\author{
Volume \\ $\left(\mathrm{ft}^{3}\right)$
}

$2,992.20$

19.47

220.87

$3,232.54$
Activity

(curies)

31.74

33.77

66.54
VOLUME PERCENTAGE BY DISPOSAL SITE (Percentages $<.1 \%$ are not displayed)

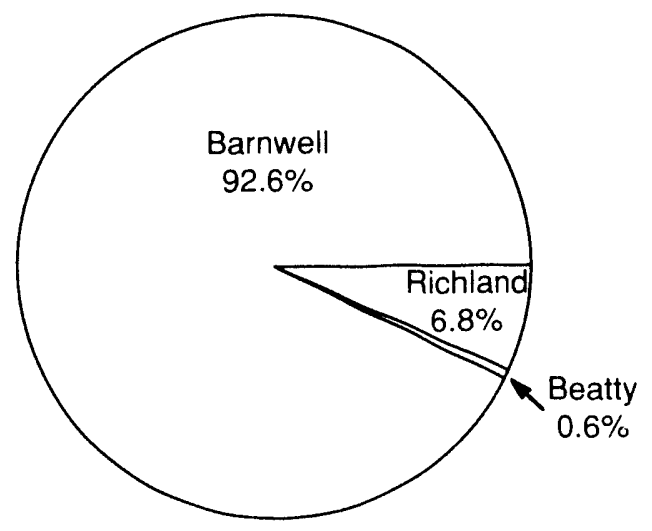

ACTIVITY PERCENTAGE BY DISPOSAL SITE

(Percentages <.1\% are not displayed)

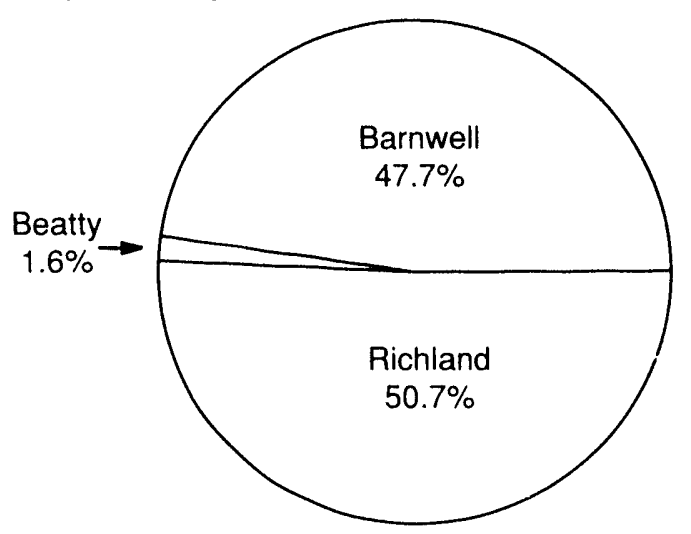

Volume Percentage by Waste Class for Direct Shipments and Total Non-Direct Volume Percentage (Percentages <.1\% are not displayed)
Direct Shipment

Waste Class

A

B

C

Total

Non-Direct Shipments

Grand Total

Total Direct Shipment

Activity (curies)

Total Non-Direct Shipment

Activity (curies)

Grand Total (curies)

6.44

$3,232.54$

34.81

Volume $\left(\mathrm{ft}^{3}\right)$

$2,851.80$

0.00

84.30

$2,936.10$

296.44

66.54

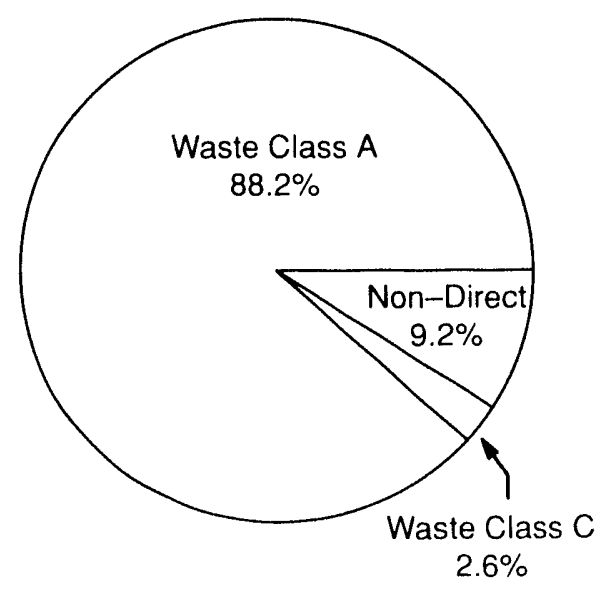




\section{LOUISIANA \\ Low-Level Radioactive Wastes Received at Commercial Disposal Sites}

\begin{tabular}{l} 
Generator \\
Category \\
\hline
\end{tabular}

Academic

Government

Industrial

Medical

Utility

Total

\begin{tabular}{c}
$\begin{array}{c}\text { Volume } \\
\left(\mathrm{ft}^{3}\right)\end{array}$ \\
\hline
\end{tabular}

32.58

0.10

375.96

21.00

$10,899.66$

$11,329.30$
Activity

(curies)

0.47

$<.01$

0.25

$<.01$

993.36

994.08
VOLUME PERCENTAGE BY SOURCE

(Percentages <. $1 \%$ are not displayed)

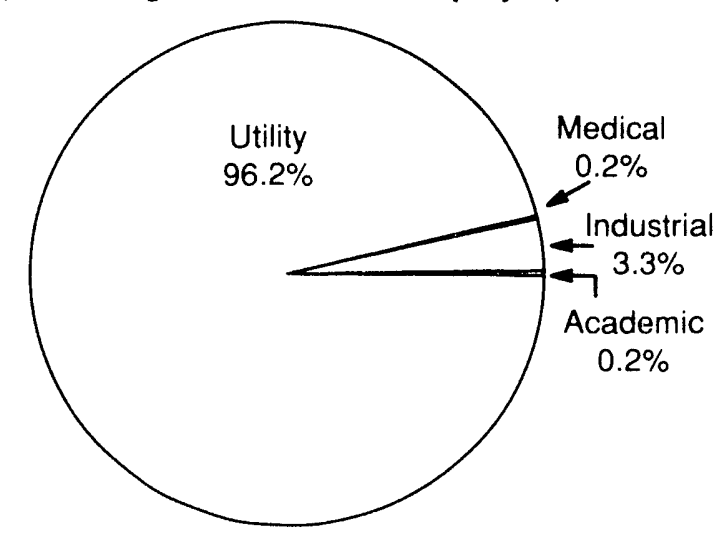

ACTIVITY PERCENTAGE BY SOURCE

(Percentages <. $1 \%$ are not displayed

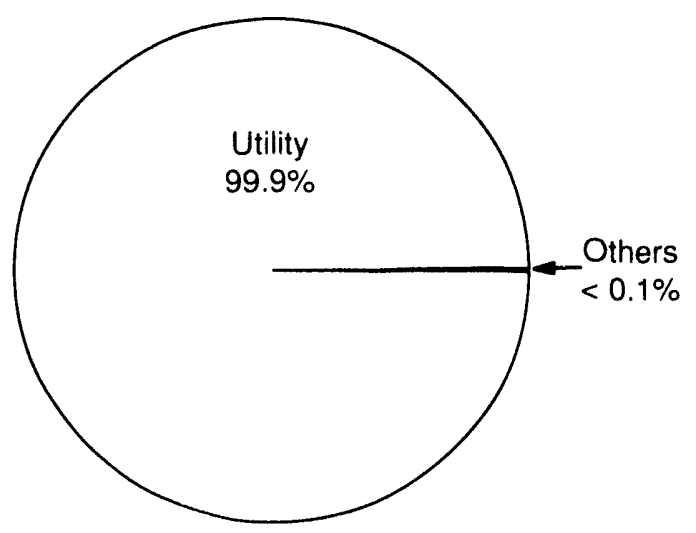




\section{LOUISIANA \\ Low-Level Radioactive Wastes Received at Commercial Disposal Sites}

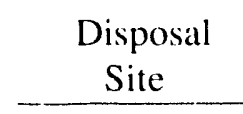

Barnwell

Beatty

Richland

Total

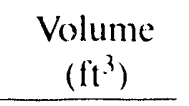

$$
\begin{array}{r}
10,902.46 \\
9.90 \\
416.94
\end{array}
$$

$11,329.30$
Activity

(curies)

993.36

$<.01$

0.72

994.08
VOLUME PERCENTAGE BY DISPOSAL SITE (Percentages <. $1 \%$ are not displayed)

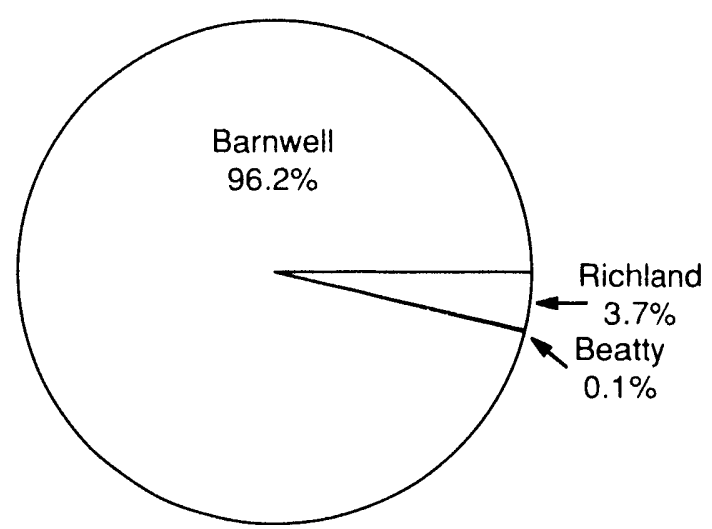

ACTIVITY PERCENTAGE BY DISPOSAL SITE (Percentages <. $1 \%$ are not displayed)

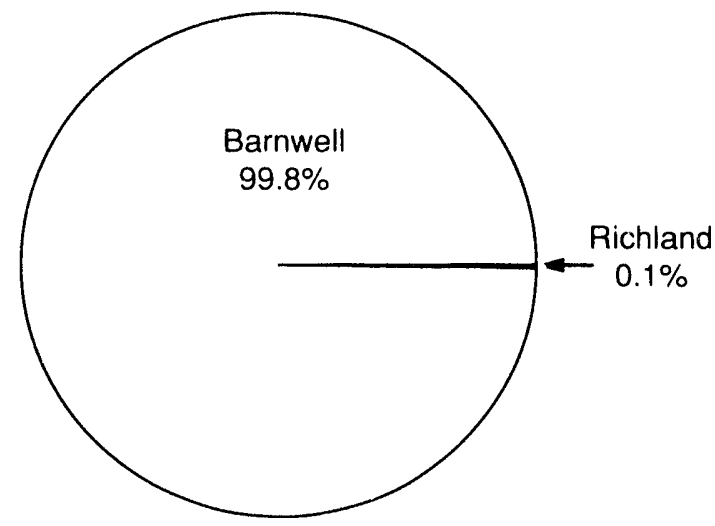

Volume Percentage by Waste Class for Direct Shipments and Total Non-Direct Volume Percentage (Percentages $<.1 \%$ are not displayed)
Direct Shipment

Waste Class

A

B

C

Total

Non-Direct Shipments

Grand Total

Total Direct Shipment

Activity (curies)

Total Non-Direct Shipment

Activity (curies)

Grand Total (curies)

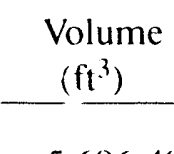

$5,696.40$

0.00

182.40

$5,878.80$

$5,450.50$

$11,329.30$

$98(0.13$

13.95

994.08

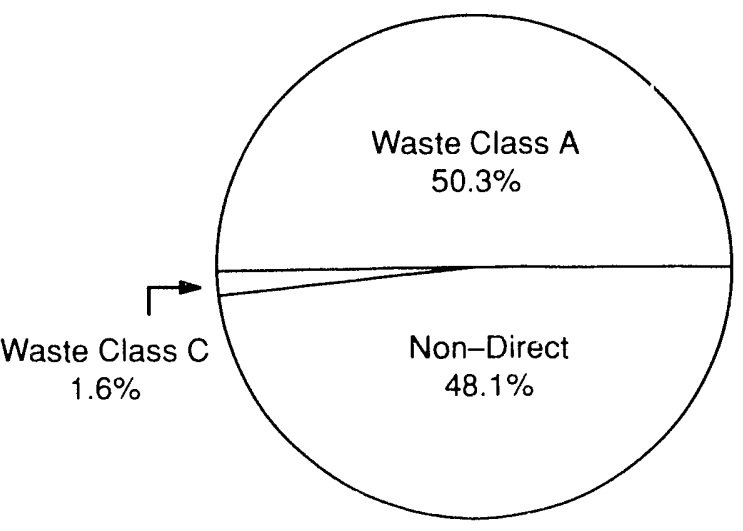




\section{NEBRASKA \\ Low-Level Radioactive Wastes Received at Commercial Disposal Sites}

\begin{tabular}{l} 
Generator \\
Category \\
\hline
\end{tabular}

Academic

Industrial

Medical

Utility

Total

Volume
$\left(\mathrm{ft}^{3}\right)$

1.10

351.15

4.52

$15,202.10$

$15,558.87$
Activity

(curies)

$<.01$

1.49

0.72

375.66

377.88
VOLUME PERCENTAGE BY SOURCE

(Percentages <. $1 \%$ are not displayed

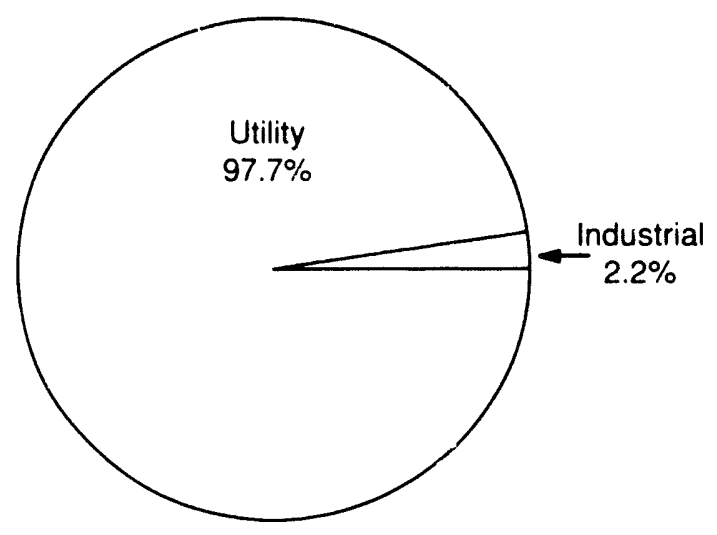

ACTIVITY PERCENTAGE BY SOURCE

(Percentages <. $1 \%$ are not displayed)

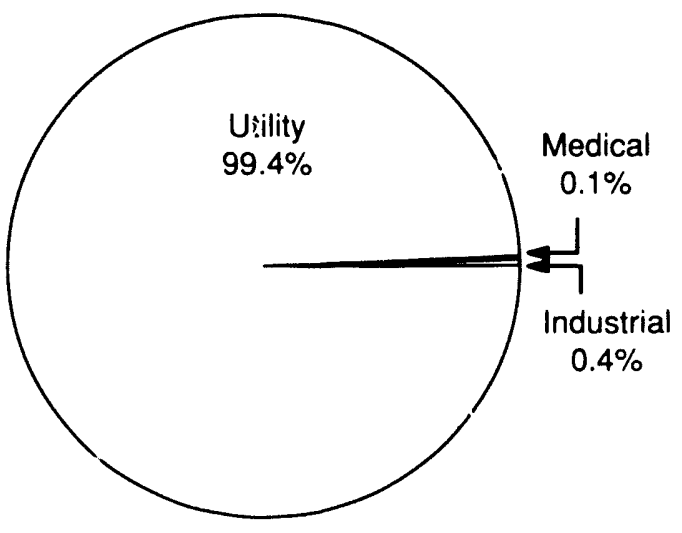




\section{NEBRASKA \\ Low-Level Radioactive Wastes Received at Commercial Disposal Sites}

\begin{tabular}{l}
\multicolumn{1}{c}{$\begin{array}{c}\text { Disposal } \\
\text { Site }\end{array}$} \\
\hline Barnwell \\
Beatty \\
Richland \\
Total
\end{tabular}

VOLUME PERCENTAGE BY DISPOSAL SITE

(Percentages $<.1 \%$ are not displayed)

$$
\text { Beatty }
$$

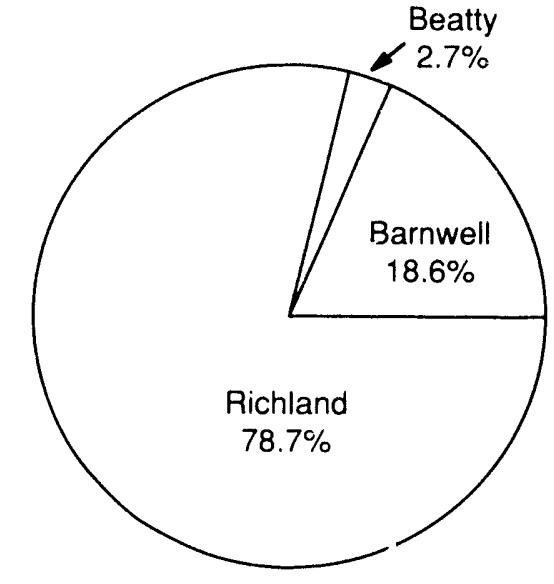

Direct Shipment

$$
\text { Waste Class }
$$

A

B

C

Total

Non-Direct Shipments

Grand Total

Total Direct Shipment

Activity (curies)

Total Non-Direct Shipment

Activity (curies)

Grand Total (curies)

\author{
Volume \\ $\left(\mathrm{ft}^{3}\right)$ \\ $2,899.60$ \\ 414.35 \\ $12,244.92$
}

$15,558.87$

\section{Activity \\ (curies)}

5.84

143.91

228.13

377.88
374.36

ACTIVITY PERCENTAGE BY DISPOSAL SITE

(Percentages $<.1 \%$ are not displayed)

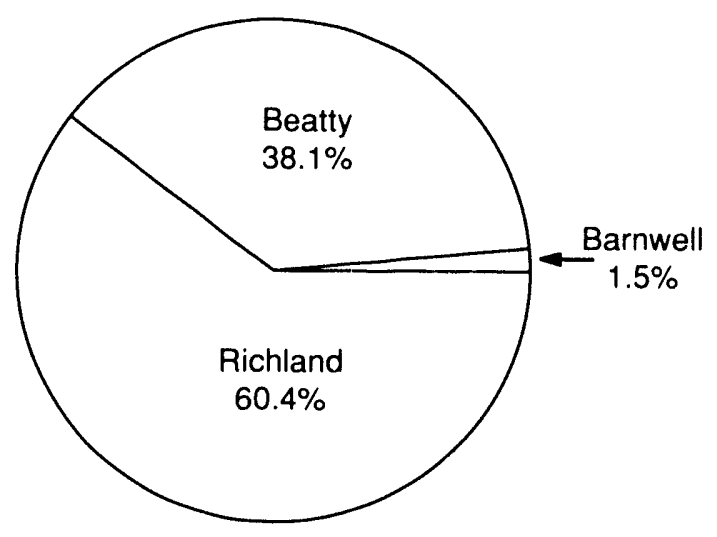

Volume Percentage by Waste Class for Direct Shipments and Total Non-Direct Volume Percentage (Percentages $<.1 \%$ are not displayed)

Volume

$\begin{array}{r}\left(\mathrm{ft}^{3}\right) \\ \hline 12,468.70 \\ 150.00 \\ 0.00 \\ \hline 12,618.70 \\ 2.940 .17 \\ \hline 15.558 .87\end{array}$

377.88

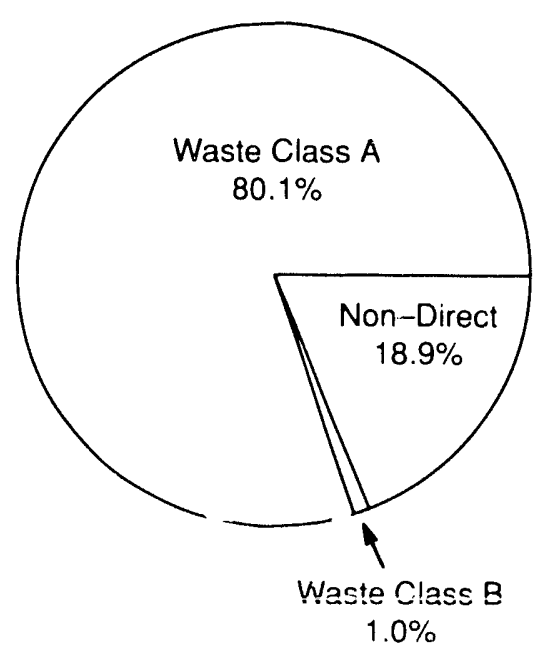




\section{OKLAHOMA \\ LovJ-Level Radioactive Wastes Received at Commercial Disposal Sites}

\begin{tabular}{l} 
Generator \\
Category \\
\hline
\end{tabular}

Academic

Government

Industrial

Medical

Total

\section{Volume}

$\left(\mathrm{ft}^{3}\right)$

122.92

37.50

$21,798.00$

9.43

$21,967.85$
Activity (curies)

$\begin{array}{r}0.15 \\ 2.12 \\ 0.77 \\ 0.01 \\ \hline 3.05\end{array}$

VOLUME PERCENTAGE BY SOURCE (Percentages <. $1 \%$ are not displayed)

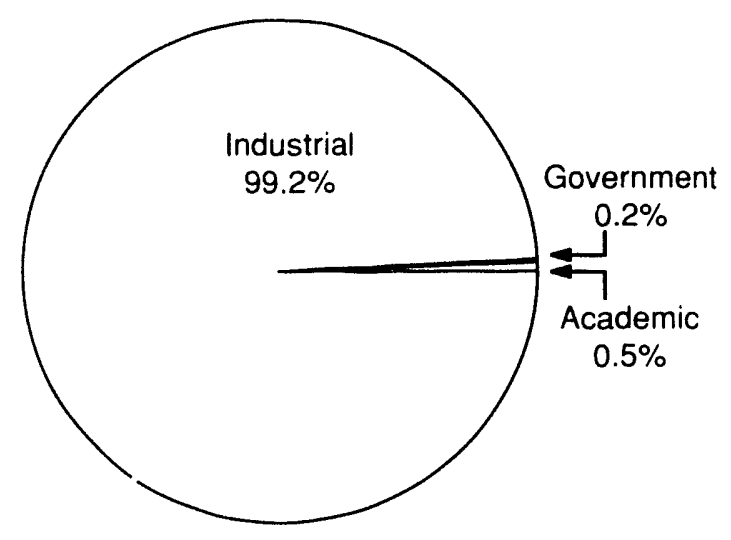

ACTIVITY PERCENTAGE BY SOURCE (Percentages <. 1\% are not displayed)

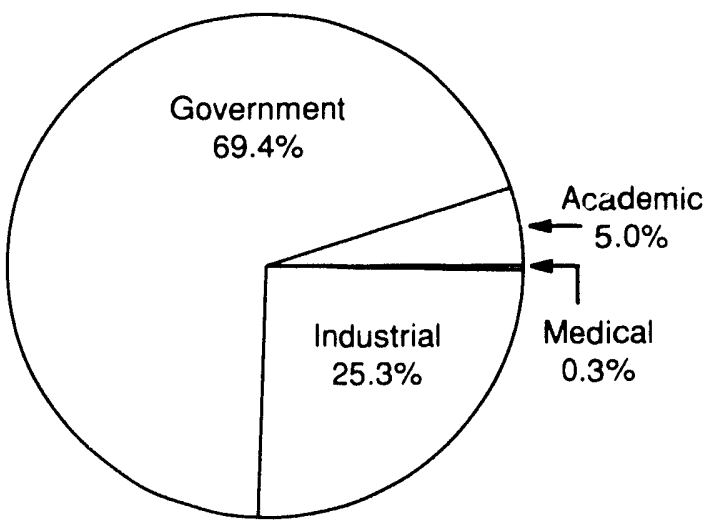




\section{OKLAHOMA \\ Low-Level Radioactive Wastes Received at Commercial Disposal Sites}

\begin{tabular}{c} 
Disposal \\
Site \\
\hline
\end{tabular}

Barnwell

Beatty

Richland

Total

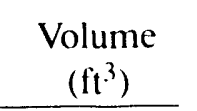

$19,718.86$

37.50

$2,211.49$

$21,967.85$
Activity

(curies)

0.45

2.12

0.48

3.05
VOLUME PERCENTAGE BY DISPOSAL SITE

(Percentages <. $1 \%$ are not displayed)

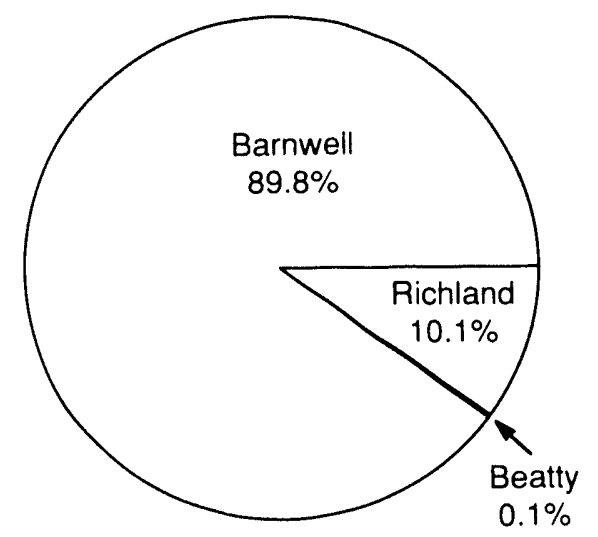

ACTIVITY PERCENTAGE BY DISPOSAL SITE

(Percentages <. $1 \%$ are not displayed)

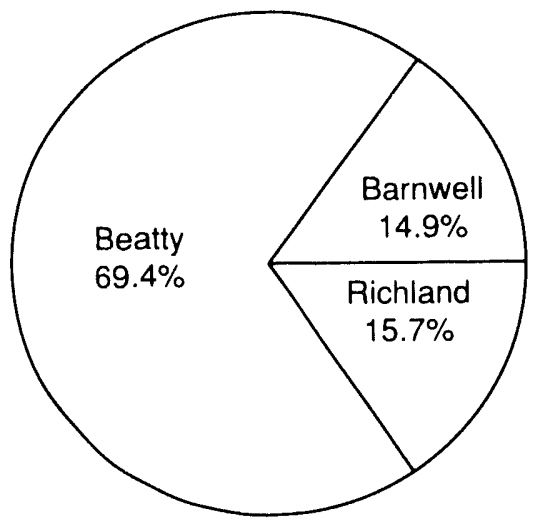

Volume Percentage by Waste Class for Direct Shipments and Total

Non-Direct Volume Percentage

(Percentages $<.1 \%$ are not displayed)

Direct Shipment

Volume

Waste Class

$\left(\mathrm{ft}^{3}\right)$

A

$21,786.40$

0.00

0.00

$21,786.40$

Total

$\frac{181.45}{21.967 .85}$

21.967 .85

0.73

Activity (curies)

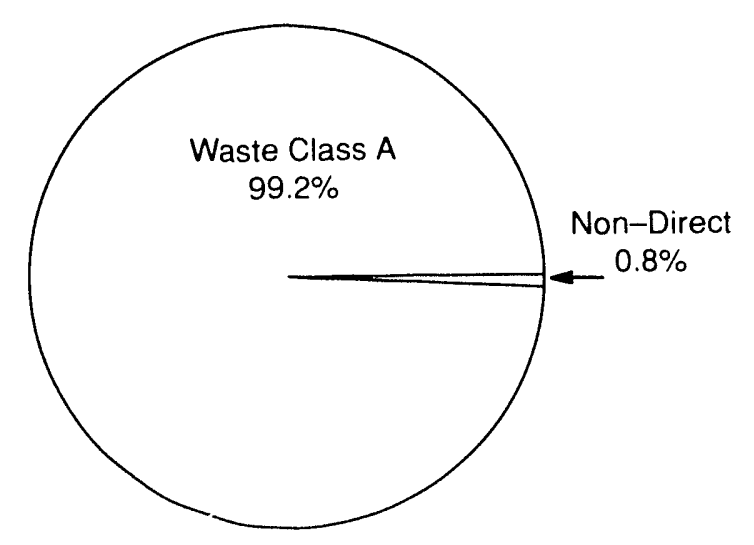

Total Non-Direct Shipment

Activity (curies)

Grand Totai icuries) 
I

I

I

I

I

I

I

I

I

I

I

I

I

I

I

I

I

I

I 


\section{CENTRAL MIDWEST COMPACT Low-Level Radioactive Wastes Received at Commercial Disposal Sites}

\begin{tabular}{lrr}
\multicolumn{1}{c}{ States } & \multicolumn{1}{c}{$\begin{array}{c}\text { Volume } \\
\left(\mathrm{ft}^{3}\right)\end{array}$} & \multicolumn{1}{c}{$\begin{array}{c}\text { Activity } \\
\text { (curies) }\end{array}$} \\
Illinois & $98,360.33$ & $8,253.48$ \\
Kentucky & $4,616.06$ & 61.10 \\
Total & $102,976.39$ & $8,314.58$
\end{tabular}

VOLUME PERCENTAGE BY STATE

(Percentages $<.1 \%$ are not displayed)

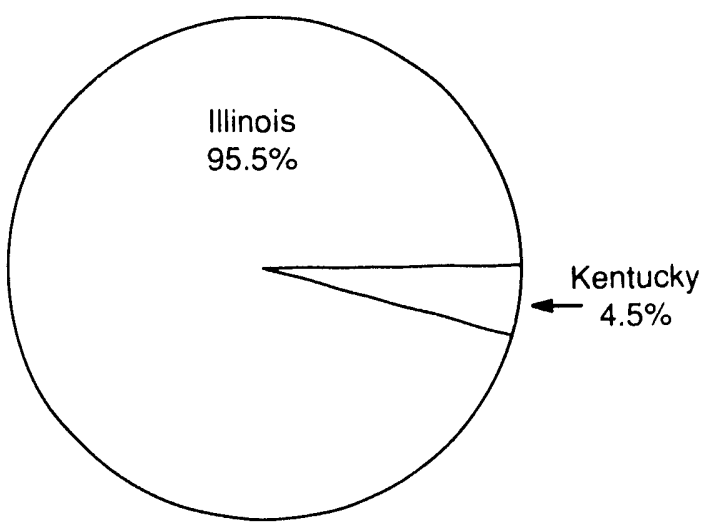

VOLUME PERCENTAGE BY CATEGORY

(Percentages $<.1 \%$ are not displayed)

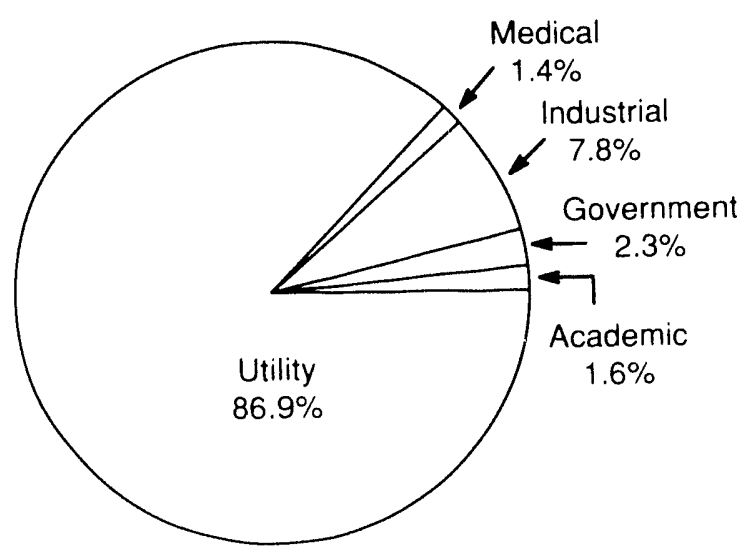




\section{ILLINOIS \\ Low-Level Radioactive Wastes Received at Commercial Disposal Sites}

\begin{tabular}{l}
\multicolumn{1}{c}{$\begin{array}{c}\text { Generator } \\
\text { Category }\end{array}$} \\
\hline Academic \\
Government \\
Industrial \\
Medical \\
Utility \\
Total
\end{tabular}

\begin{tabular}{c}
$\begin{array}{c}\text { Volume } \\
\left(\mathrm{ft}^{3}\right)\end{array}$ \\
\hline $1,248.74$ \\
$1,032.11$ \\
$5,160.23$ \\
$1,474.98$ \\
$89,444.27$ \\
\hline
\end{tabular}

Activity

(curies)

$98,360.33$

10.00

310.49

75.42

2.27

$7,855.30$

$8,253.48$

VOLUME PERCENTAGE BY SOURCE (Percentages <. $1 \%$ are not displayed)

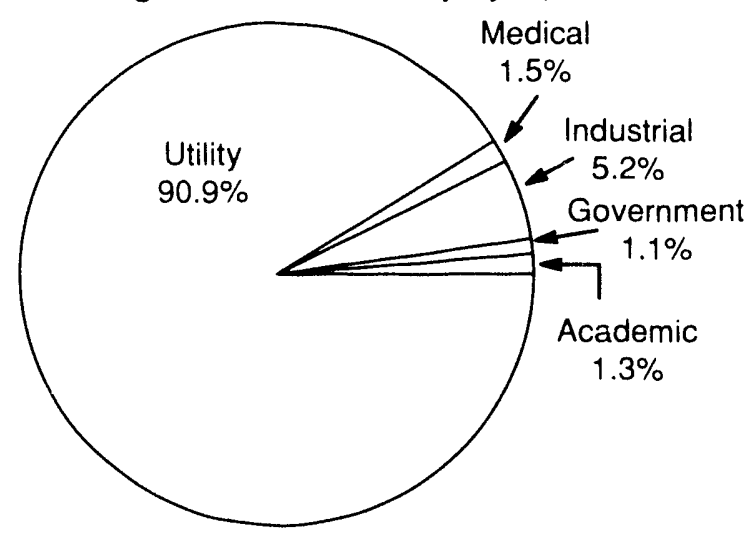

ACTIVITY PERCENTAGE BY SOURCE

(Percentages <. $1 \%$ are not displayed)

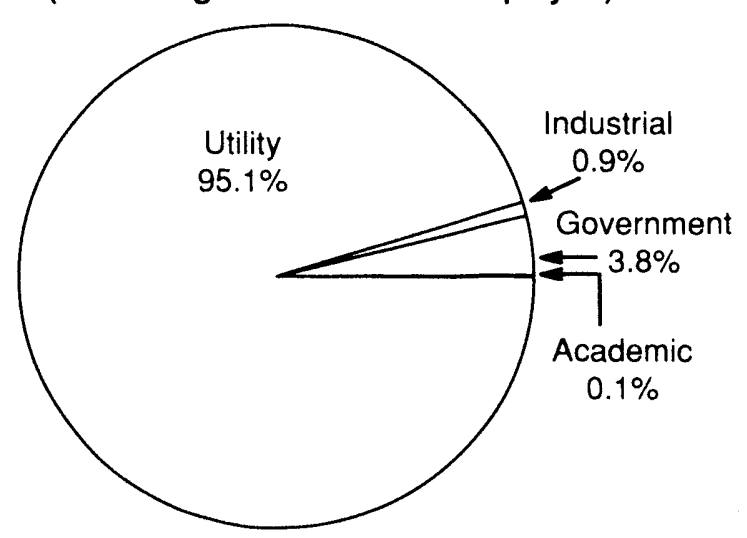




\section{ILLINOIS \\ Low-Level Radioactive Wastes Received at Commercial Disposal Sites}

\begin{tabular}{|c|c|c|}
\hline $\begin{array}{l}\text { Disposal } \\
\text { Site }\end{array}$ & $\begin{array}{c}\text { Volume } \\
\left(\mathrm{ft}^{3}\right)\end{array}$ & $\begin{array}{l}\text { Activity } \\
\text { (curies) }\end{array}$ \\
\hline Barnwell & 71.309 .81 & $5,417.81$ \\
\hline Beatty & $5,322.12$ & $2,165.92$ \\
\hline Richland & $21,728.40$ & 669.74 \\
\hline Total & $98,360.33$ & $8,253.48$ \\
\hline
\end{tabular}

VOLUME PERCENTAGE BY DISPOSAL SITE
(Percentages $<.1 \%$ are not displayed)

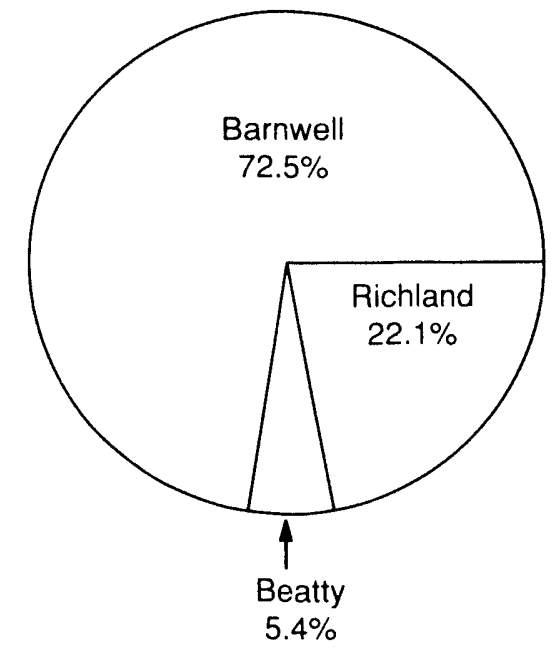

ACTIVITY PERCENTAGE BY DISPOSAL SITE

(Percentages <. $1 \%$ are not displayed)

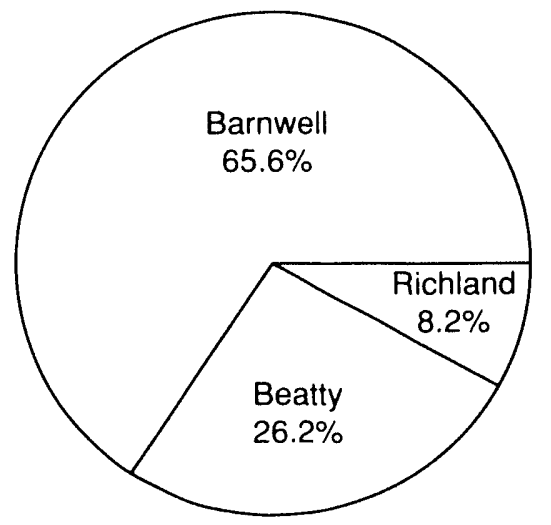

Volume Percentage by Waste Class for Direct Shipments and Total Non-Direct Volume Percentage (Percentages <. $1 \%$ are not displayed)
Direct Shipment Waste Class
A
B
C

Total

Non-Direct Shipments

Grand Total

Total Direct Shipment

Activity (curies)

Total Non-Direct Shipment

Activity (curies)

Grand Total (curies)
Volume $\left(\mathrm{ft}^{3}\right)$

49,929.25

$3,584.50)$

613.60)

54.127 .35

$44,232.98$

98.360 .33

$7,758.26$

495.22

$8,253.48$ 


\section{KENTUCKY \\ Low-Level Radioactive Wastes Received at Commercial Disposal Sites}

\begin{tabular}{l} 
Generator \\
Category \\
\hline
\end{tabular}

Academic

Government

Industrial

Total

$\begin{array}{r}\begin{array}{c}\text { Volume } \\ \left(\mathrm{ft}^{3}\right)\end{array} \\ \hline \begin{array}{r}428.35 \\ 1,299.20 \\ 2,888.51\end{array} \\ \hline 4.616 .06\end{array}$

Activity

(curies)

$\begin{array}{r}0.40 \\ 59.56 \\ 1.14 \\ \hline 61.10\end{array}$

VOLUME PERCENTAGE BY SOURCE

(Percentages <. $1 \%$ are not displayed)

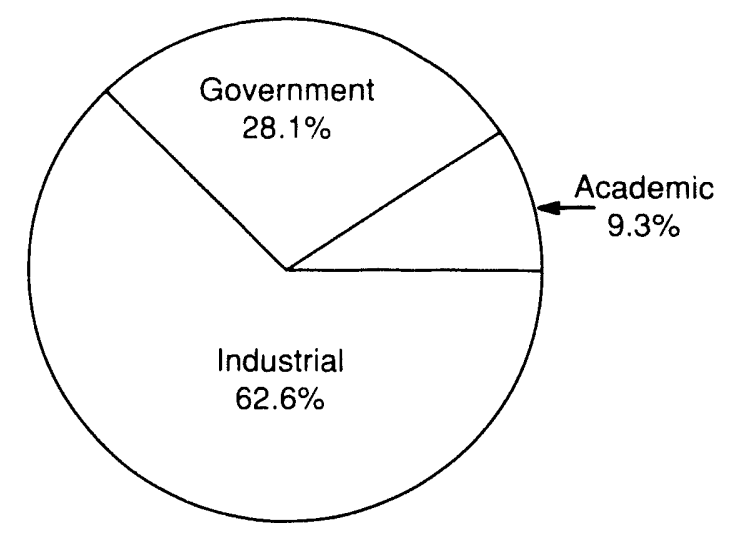

ACTIVITY PERCENTAGE BY SOURCE

(Percentages <. $1 \%$ are not displayed)

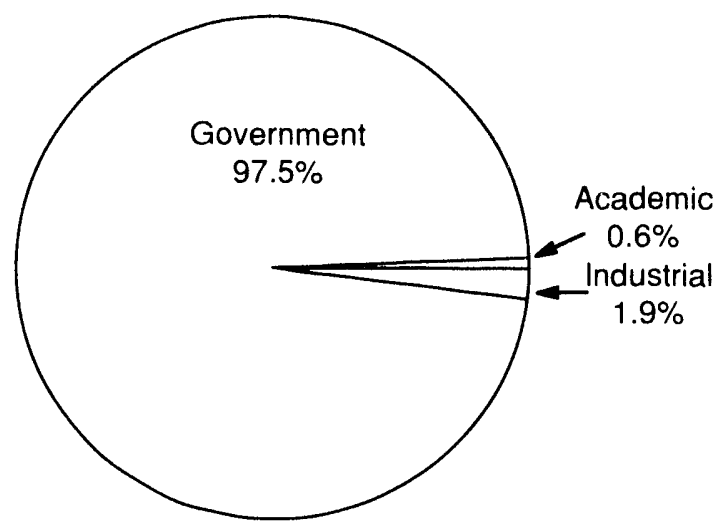




\section{KENTUCKY \\ Low-Level Radioactive Wastes Received at Commercial Disposal Sites}

\begin{tabular}{c} 
Disposal \\
Site \\
\hline
\end{tabular}

Barnwell

Beatty

Richland

Total

\begin{tabular}{c}
$\begin{array}{c}\text { Volume } \\
\left(\mathrm{ft}^{3}\right)\end{array}$ \\
\hline
\end{tabular}

$1,298.70$

12.22

$3,305.14$

$4,616.06$
Activity

(curies)

59.56

0.17

1.37

61.10
VOLUME PERCENTAGE BY DISPOSAL SITE (Percentages <. $1 \%$ are not displayed)

\section{Beatty}

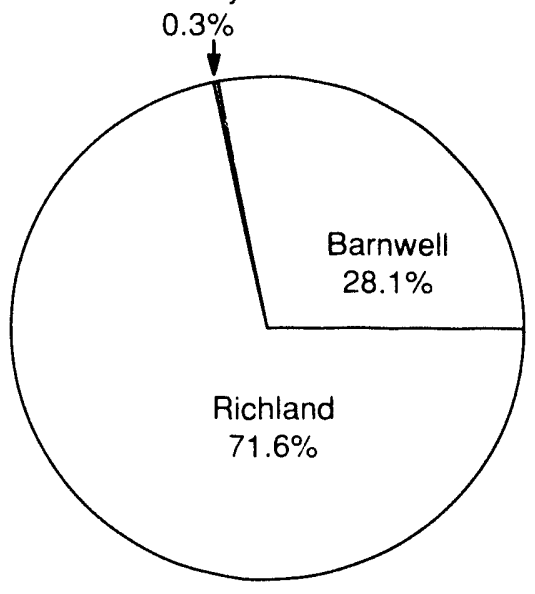

Direct Shipment

Waste Class

A

B

C

Total

Non-Direct Shipments

Grand Total

Total Direct Shipment

Activity (curies)

Total Non-Direct Shipment

Activity (curies)

Grand Total (curies)
Volume $\left(\mathrm{ft}^{3}\right)$

$1,280.00$

0.00

0.00

1.280 .00

$3,336.06$

$4,616.06$

$<.01$

61.10

61.10

ACTIVITY PERCENTAGE BY DISPOSAL SITE

(Percentages $<.1 \%$ are not displayed)

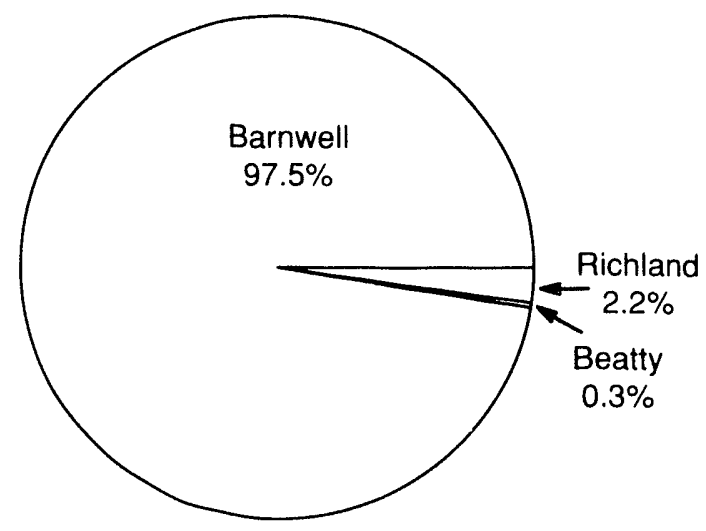

Volume Percentage by Waste Class for Direct Shipments and Total Non-Direct Volume Percentage (Percentages $<.1 \%$ are not displayed)

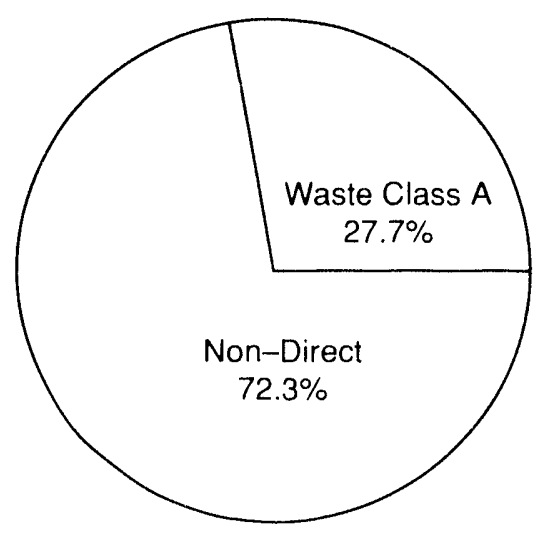




\section{MIDWEST COMPACT \\ Low-Level Radioactive Wastes Received at Commercial Disposal Sites}

\begin{tabular}{lrr}
\multicolumn{1}{c}{ States } & \multicolumn{1}{c}{$\begin{array}{c}\text { Volume } \\
\left(\mathrm{ft}^{3}{ }^{3}\right.\end{array}$} & \multicolumn{1}{c}{$\begin{array}{c}\text { Activity } \\
\text { (curies) }\end{array}$} \\
\cline { 2 - 2 } Indiana & $1,956.01$ & $\begin{array}{r} \\
\text { Iowa }\end{array}$ \\
Michigan & $5,557.86$ & $37,807.79$ \\
Minnesota & $36,038.53$ & $21,372.83$ \\
Missouri & $26,822.72$ & $1,182.61$ \\
Ohio & $19,609.59$ & 919.62 \\
Wisconsin & $24,146.85$ & $4,315.70$ \\
& $9,217.32$ & 569.68 \\
\hline Total & $123,348.88$ & $66,211.90$
\end{tabular}

VOLUME PERCENTAGE BY STATE

(Percentages $<.1 \%$ are not displayed)

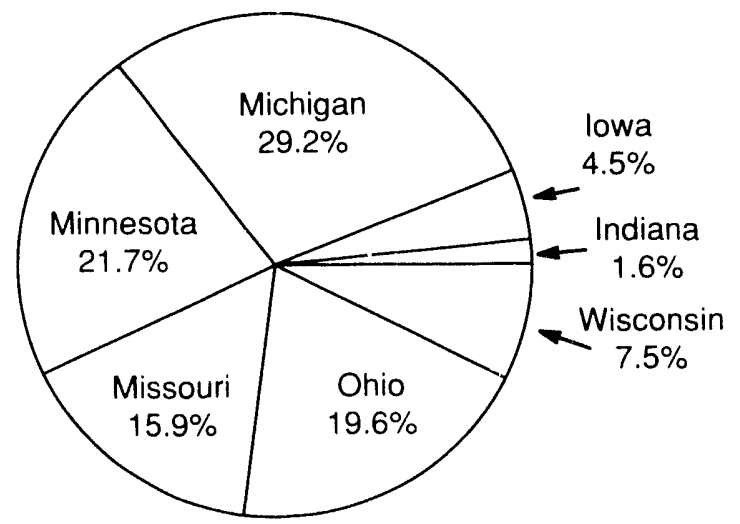

VOLUME PERCENTAGE BY CATEGORY

(Percentages <. $1 \%$ are not displayed)

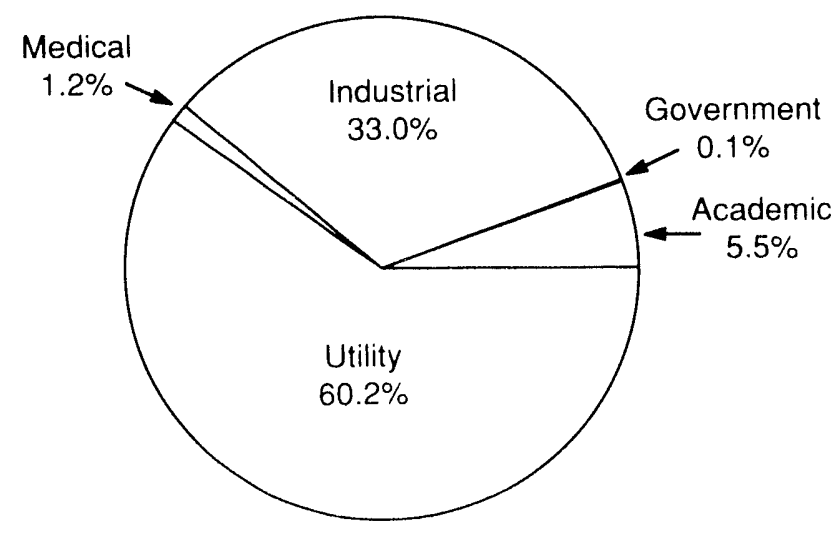




\section{IOWA \\ Low-Level Radioactive Wastes Received at Commercial Disposal Sites}

\begin{tabular}{l} 
Generator \\
Category \\
\hline
\end{tabular}

Academic

Government

In.... rial

Medical

Utility

Toin'

$\begin{array}{r}\begin{array}{c}V_{c} \text { ume } \\ \left(\mathrm{ft}^{3}\right)\end{array} \\ \hline 271.76 \\ 0.50 \\ 393.70 \\ 3.30 \\ 4,888.60 \\ \hline 5,557.86\end{array}$

Activity

(curies)

0.05

0.01

0.51

0.09

$37,807.13$

37.807 .79
VOLUME PERCENTAGE BY SQURCE (Percentages $<.1 \%$ are not displayed)

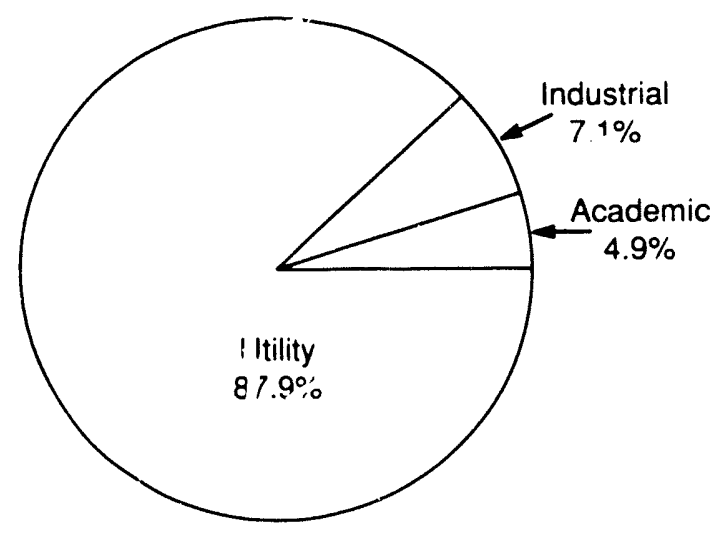

ACTIVITY PERCENTAGE BY SOURCE

(Per. 7 tages <. $1 \%$ are not displayed)

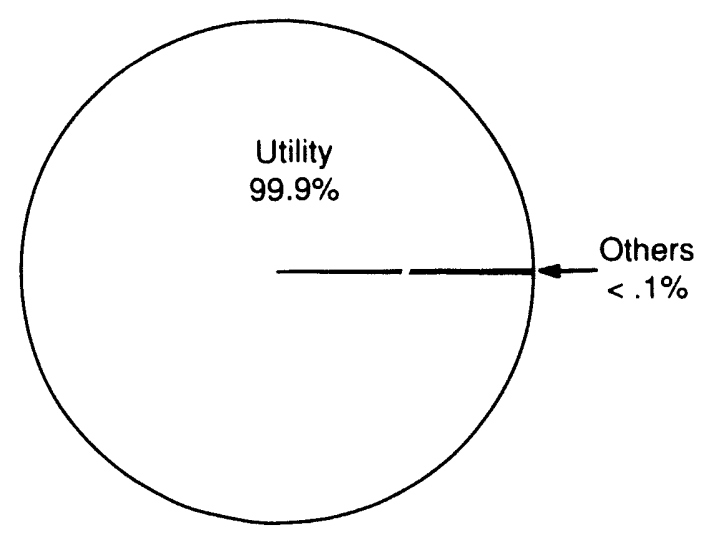




\section{IOWA \\ Low-Level Radioactive Wastes Received at Commercial Disposal Sites}

\begin{tabular}{c} 
Disposal \\
Site \\
\hline
\end{tabular}

Barnwell

Beatty

Richland

Total

\section{Volume}

$\left(\mathrm{ft}^{3}\right)$

$$
\begin{array}{r}
4,512.50 \\
158.20 \\
887.16 \\
\hline
\end{array}
$$

$5,557.86$
Activity

(curies)

$37,354.85$

0.62

452.33

$37,807.79$
VOLUME PERCENTAGE BY DISPOSAL SITE (Percentages <.1\% are not displayed)

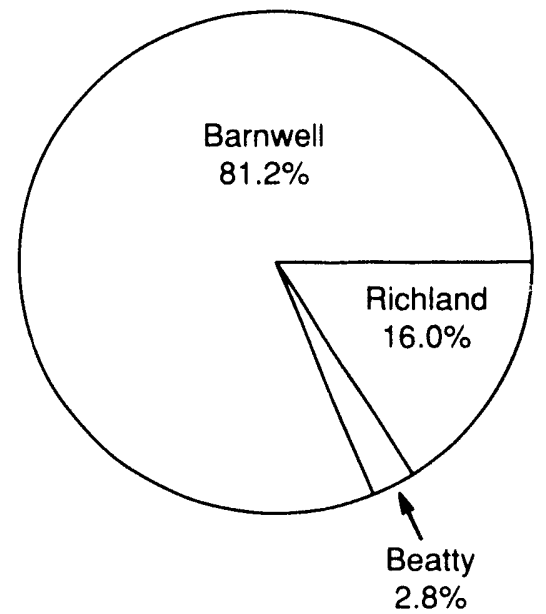

\section{ACTIVITY PERCENTAGE BY DISPOSAL SITE (Percentages <. $1 \%$ are not displayed)}

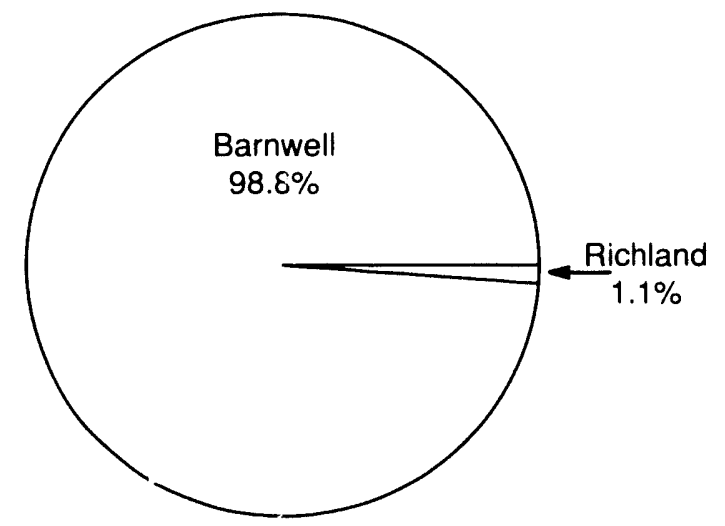

Volume Percentage by Waste Class for Direct Shipments and Total Non-Direct Volume Percentage (Percentages $<.1 \%$ are not displayed)
Direct Shipment

Waste Class

A
B
C

Total

Non-Direct Shipments

Grand Total

Total Direct Shipment

Activity (curies)

Total Non-Direct Shipment

Activity (curies!

Graind Total (curies)

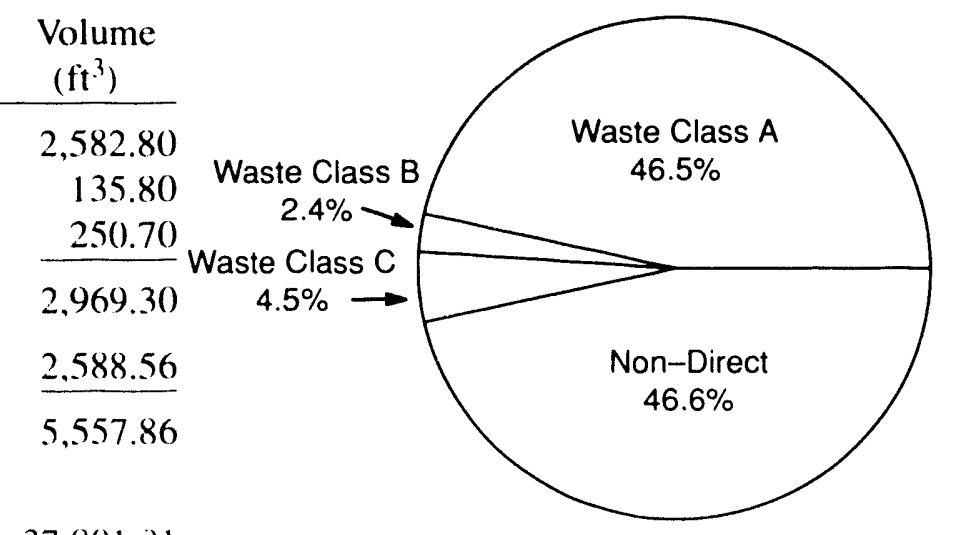

37.8() 101

$\frac{6.78}{37.807 .79}$ 


\section{INDIANA \\ Low-Level Radioactive Wastes Received at Commercial Disposal Sites}

\section{Generator \\ Category}

Academic

Government

Industrial

Medical

Utility

Total

$\begin{array}{r}\begin{array}{c}\text { Volume } \\ \left(\mathrm{ft}^{3}\right)\end{array} \\ \hline 533.28 \\ 2.00 \\ 1,353.42 \\ 27.87 \\ 39.44 \\ \hline 1,956.01\end{array}$

Activity

(curies)

22.89

11.25

8.35

0.30

0.87

43.67
VOLUME PERCENTAGE BY SOURCE

(Percentages <. $1 \%$ are not displayed)
ACTIVITY PERCENTAGE BY SOURCE

(Percentages <. $1 \%$ are not displayed)
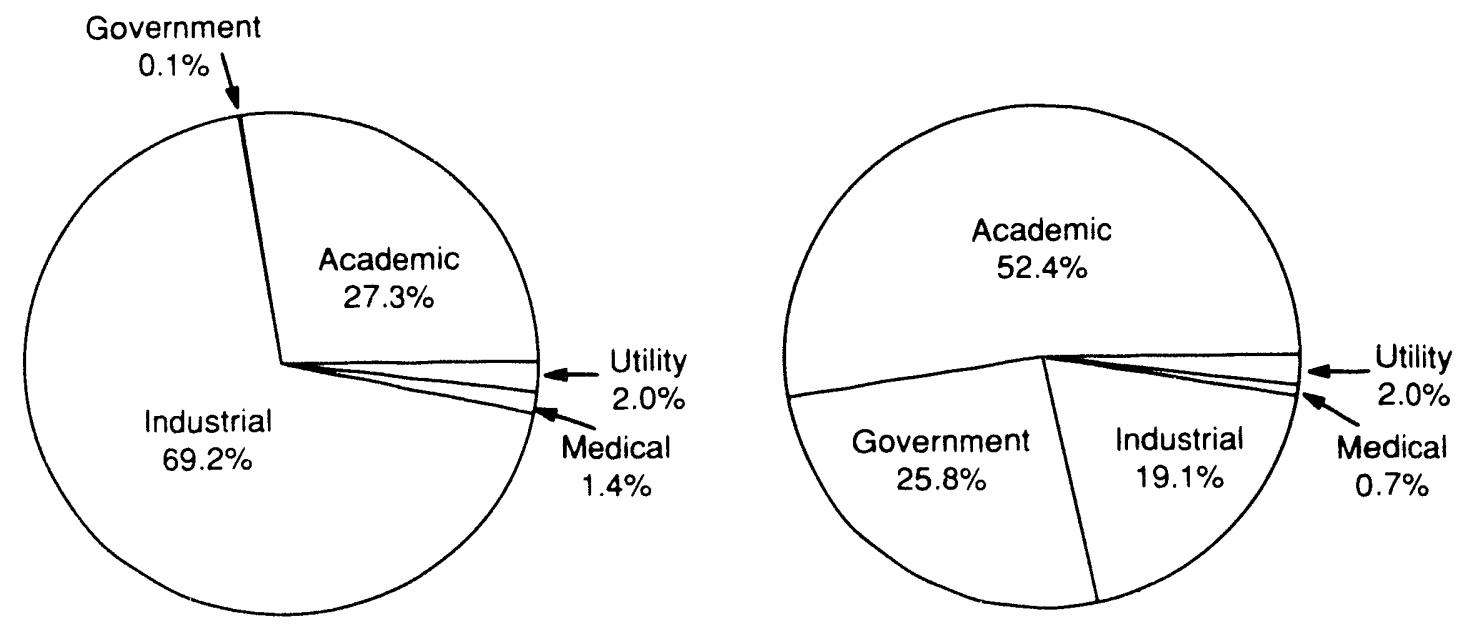


\section{INDIANA \\ Low-Level Radioactive Wastes Received at Commercial Disposal Sites}

\begin{tabular}{lrr}
\multicolumn{1}{c}{$\begin{array}{c}\text { Disposal } \\
\text { Site }\end{array}$} & \multicolumn{1}{c}{$\begin{array}{c}\text { Volume } \\
\left(\mathrm{ft}^{3}\right)\end{array}$} & \multicolumn{1}{c}{$\begin{array}{c}\text { Activity } \\
\text { (curies) }\end{array}$} \\
Barnwell & $1,579.30$ & 16.27 \\
Beatty & 107.02 & 27.17 \\
Richland & 269.69 & 0.23 \\
Total & $1,956.01$ & 43.67
\end{tabular}

VOLUME PERCENTAGE BY DISPOSAL SITE (Percentages <.1\% are not displayed)

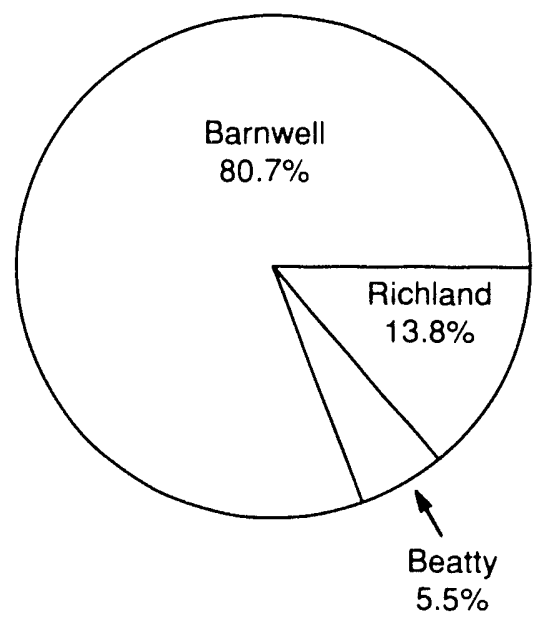

Direct Shipment

Waste Class

$$
\begin{aligned}
& \text { A } \\
& \text { B } \\
& \text { C }
\end{aligned}
$$

Total

Non-Direct Shipments

Grand Total

Total Direct Shipment

Activity (curies)

Total Non-Direct Shipment

Activity (curies)

Grand Total (curies) $\left(\mathrm{ft}^{3}\right)$
ACTIVITY PERCENTAGE BY DISPOSAL SITE (Percentages $<.1 \%$ are not displayed)

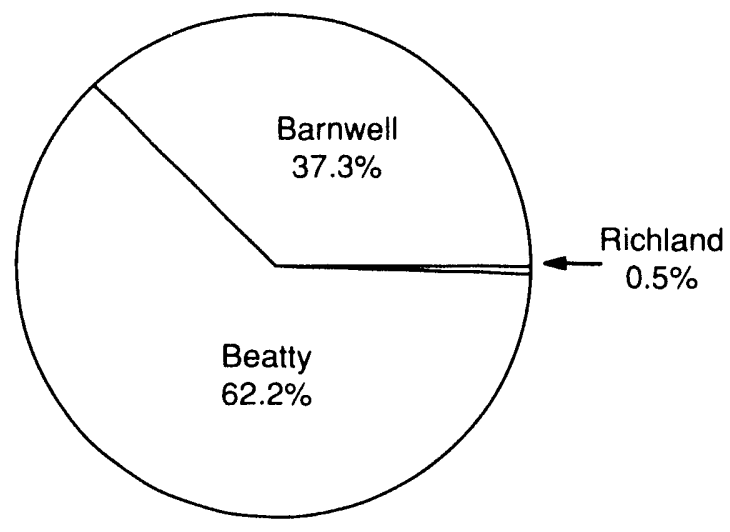

Volume Percentage by Waste Class for Direct Shipments and Total Non-Direct Volume Percentage (Percentages <. $1 \%$ are not displayed)

Volume

$1,192.20$

0.00

0.00

$1,192.20$

763.81

$1,956.01$

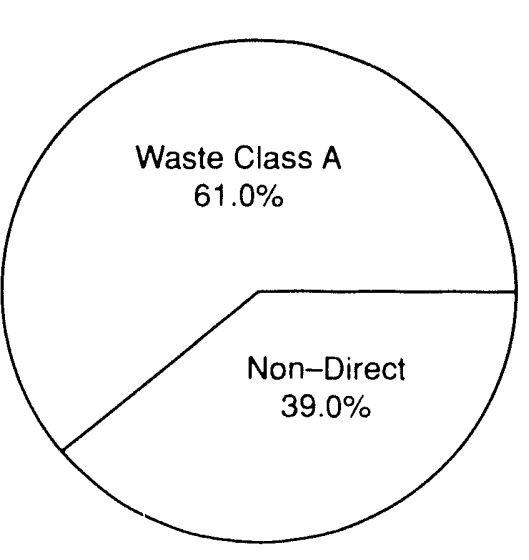

39.13

43.67 


\section{MICHIGAN \\ Low-Level Radioactive Wastes Received at Commercial Disposal Sites}

\section{Generator \\ Category}

Academic

Government

Industrial

Medical

Utility

Total
Volume

$\left(\mathrm{ft}^{3}\right)$

$1,658.45$

39.77

$1,104.03$

374.38

$32,861.90$

$36,038.53$
Activity

(curies)

64.16

17.51

35.86

0.47

$21,254.84$

$21,372.83$
VOLUME PERCENTAGE BY SOURCE (Percentages <. $1 \%$ are not displayed)
ACTIVITY PERCENTAGE BY SOURCE

(Percentages <. $1 \%$ are not displayed)
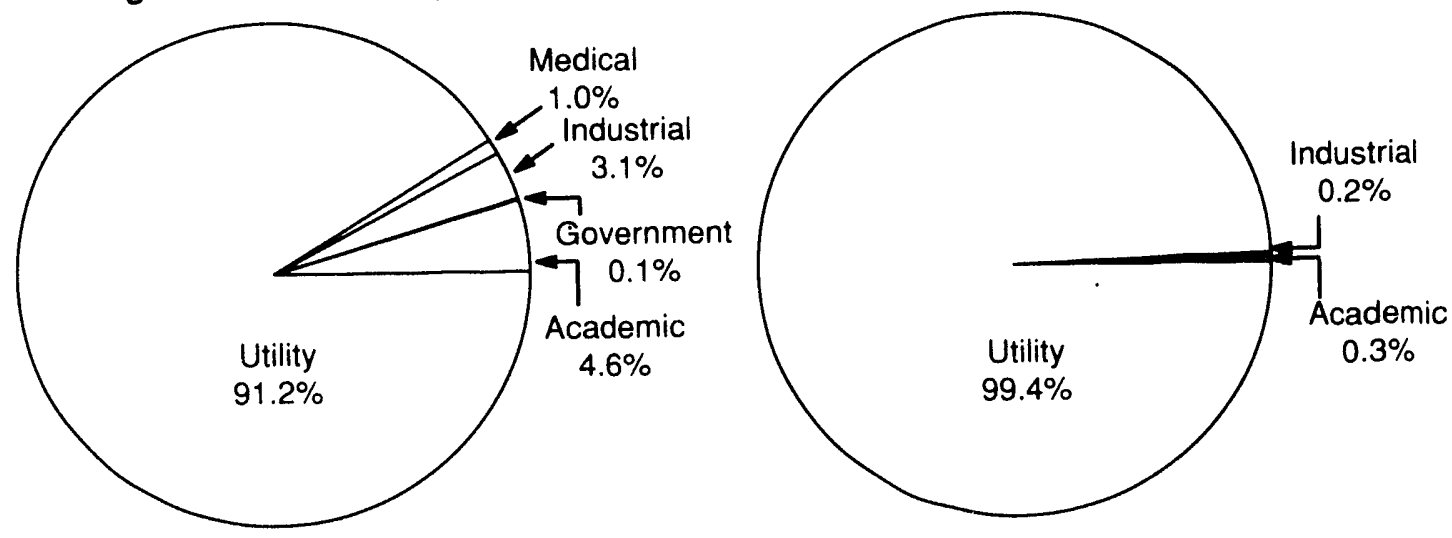


\section{MICHIGAN \\ Low-Level Radioactive Wastes Received at Commercial Disposal Sites}

\begin{tabular}{c} 
Disposal \\
Site \\
\hline
\end{tabular}

Barnwell

Beatty

Richland

Total

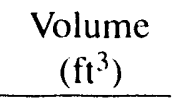

$27,509.59$

459.64

$8,069.30$

$36,038.53$
Activity

(curies)

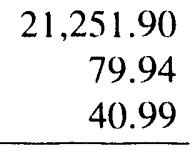

$21,372.83$
VOLUME PERCENTAGE BY DISPOSAL SITE

(Percentages $<.1 \%$ are not displayed)

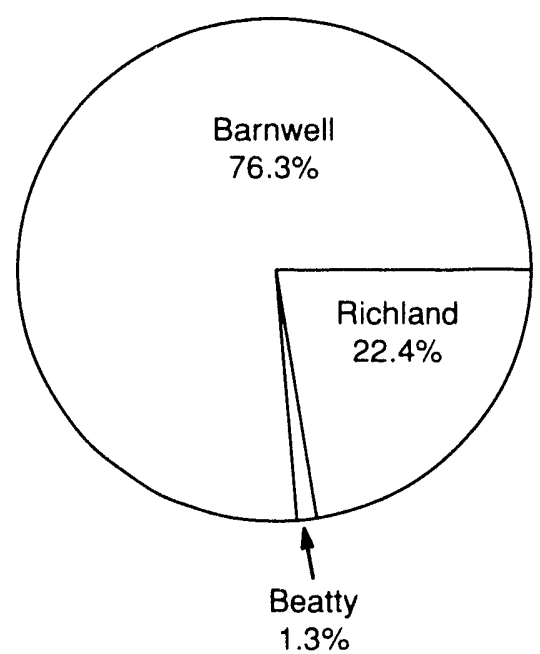

ACTIVITY PERCENTAGE BY DISPOSAL SITE

(Percentages <. $1 \%$ are not displayed)

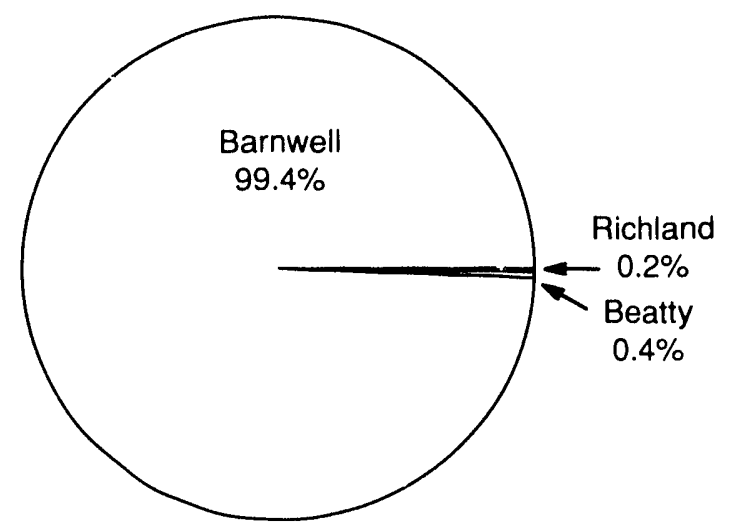

Volume Percentage by Waste Class for Direct Shipments and Total Non-Direct Volume Percentage (Percentages $<.1 \%$ are not displayed)
Direct Shipment

Waste Class

$$
\begin{aligned}
& \text { A } \\
& \text { B } \\
& \text { C }
\end{aligned}
$$

Total

Non-Direct Shipments

Grand Total

Total Direct Shipment Activity (curies)

Total Non-Direct Shipment

Activity (curies)

Grand Total (curies)
Volume $\left(\mathrm{ft}^{3}\right)$

$19,751.50$

$1,085.00$

114.80

$20,951.30$

$15,087.23$

36.038 .53

$21,180.04$

192.79

$21,372.83$ 


\section{MINNESOTA \\ Low-Level Radioactive Wastes Received at Commercial Disposal Sites}

\begin{tabular}{lrr}
$\begin{array}{l}\text { Generator } \\
\text { Category }\end{array}$ & \multicolumn{1}{c}{$\begin{array}{c}\text { Volume } \\
\left(\mathrm{ft}^{3}\right)\end{array}$} & $\begin{array}{r}\text { Activity } \\
\text { (curies) }\end{array}$ \\
\cline { 2 - 2 } Academic & 23.92 & 0.05 \\
Government & 23.00 & 8.00 \\
Industrial & $22,221.52$ & 153.14 \\
Medical & 887.48 & 2.92 \\
Utility & $3,709.00$ & $1,019.69$ \\
\hline & $26,864.92$ & $1,183.80$
\end{tabular}

VOLUME PERCENTAGE BY SOURCE (Percentages <. $1 \%$ are not displayed)

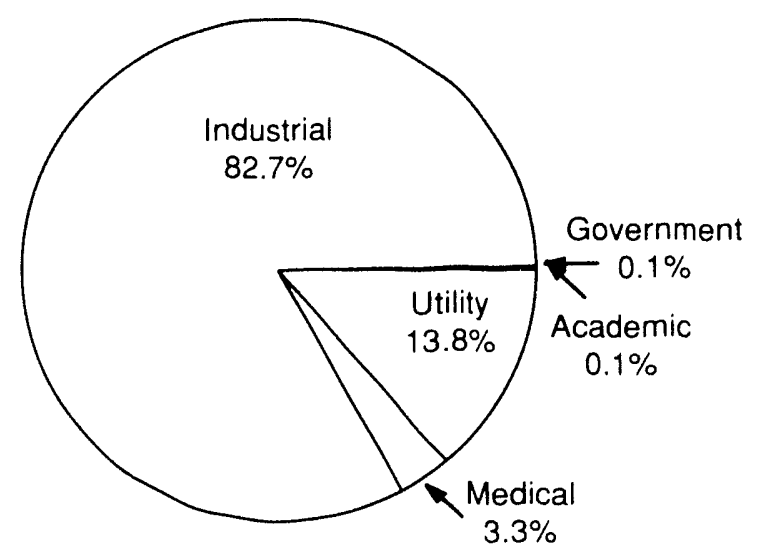

ACTIVITY PERCENTAGE BY SOURCE (Percentages $<.1 \%$ are not displayed)

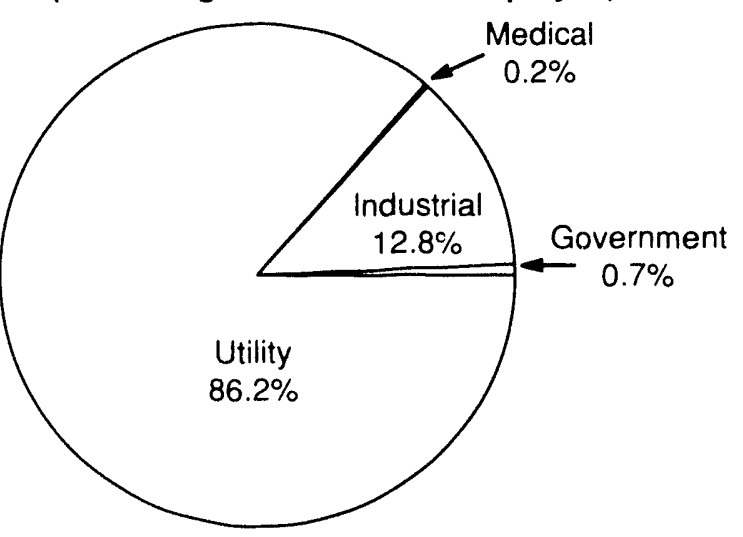




\section{MINNESOTA \\ Low-Level Radioactive Wastes Received at Commercial Disposal Sites}

\begin{tabular}{c} 
Disposal \\
Site \\
\hline
\end{tabular}

Barnwell

Beatty

Richland

Total

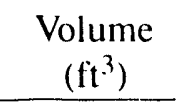

$3,118.04$

207.30

$23,539.58$

$26,864.92$
Activity

(curies)

927.51

151.32

104.98

$1,183.80$
VOLUME PERCENTAGE BY DISPOSAL SITE (Percentages <.1\% are not displayed)

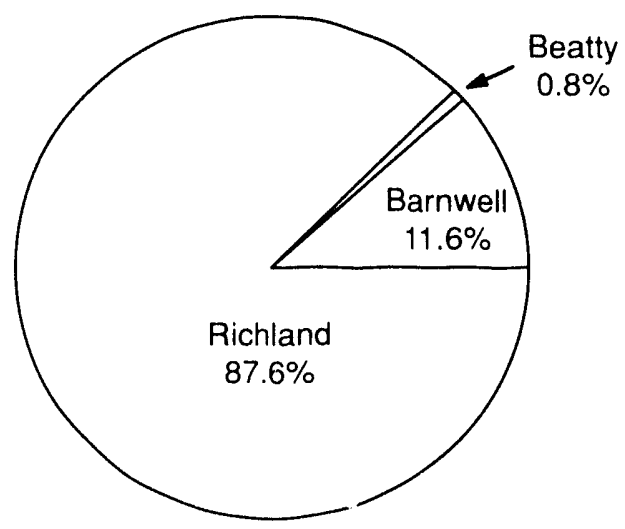

Direct Shipment

Waste Ciass

A

B

C

Total

Non-Direct Shipments

Grand Total

Total Direct Shipment

Activity (curies.)

Total Non-Dircct Shipment

Activity (curies)

Grand Total (curies)
Volume

$\left(\mathrm{ft}^{3}\right)$

$23,415.60$

527.70

0.00

$23,943.30$

$2,921.62$

$26,864.92$

$1,019.63$

164.17

$1,183.80$
ACTIVITY PERCENTAGE BY DISPOSAL SITE (Percentages <. $1 \%$ are not displayed)

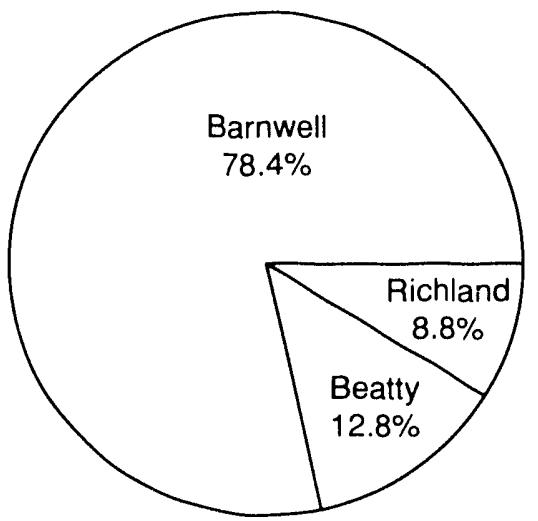

Volume Percentage by Waste Class

for Direct Shipments and Total

Non-Direct Volume Percentage

(Percentages $<.1 \%$ are not displayed)

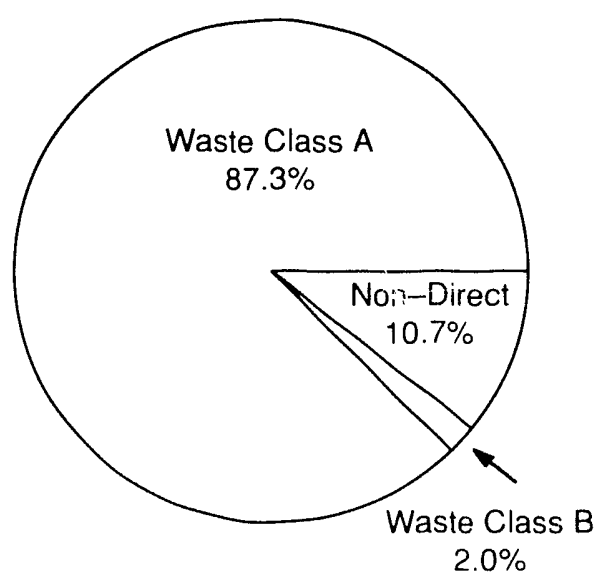




\section{MISSOURI \\ Low-Level Radioactive Wastes Received \\ at Commercial Disposal Sites}

\author{
Generator \\ Category \\ Academic \\ Government \\ Industrial \\ Medical \\ Utility
}

Total

VOLUME PERCENTAGE BY SOURCE (Percentages $<.1 \%$ are not displayed)

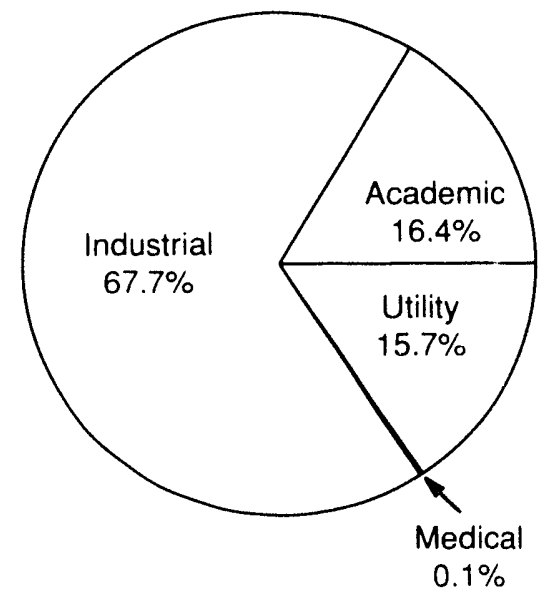

$\begin{array}{r}\begin{array}{c}\text { Volume } \\ \left(\mathrm{ft}^{3}\right)\end{array} \\ \hline 3,221.49 \\ 2.00 \\ 13,281.80 \\ 29.90 \\ 3,074.40 \\ \hline\end{array}$

$19,609.59$

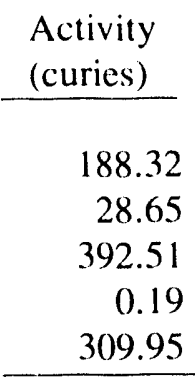

919.62

ACTIVITY PERCENTAGE BY SOURCE (Percentages $<.1 \%$ are not displayed)

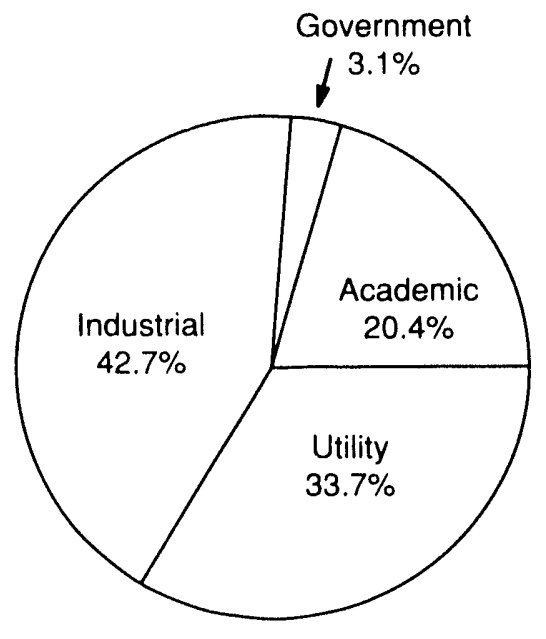




\section{MISSOURI \\ Low-Level Radioactive Wastes Received at Connmercial Disposal Sites}

\begin{tabular}{lrr}
\multicolumn{1}{c}{$\begin{array}{c}\text { Disposal } \\
\text { Site }\end{array}$} & \multicolumn{1}{c}{$\begin{array}{c}\text { Volume } \\
\left(\mathrm{ft}^{3}{ }^{3}\right)\end{array}$} & $\begin{array}{c}\text { Activity } \\
\text { (curies) }\end{array}$ \\
Barnwell & $13,095.63$ & $\begin{array}{r}467.67 \\
\text { Beatty }\end{array}$ \\
Richland & $1,218.71$ & 392.53 \\
Total & $5,295.25$ & 59.42 \\
\hline & $19,609.59$ & 919.62
\end{tabular}

VOLUME PERCENTAGE BY DISPOSAL SITE

(Percentages <. $1 \%$ are not displayed)

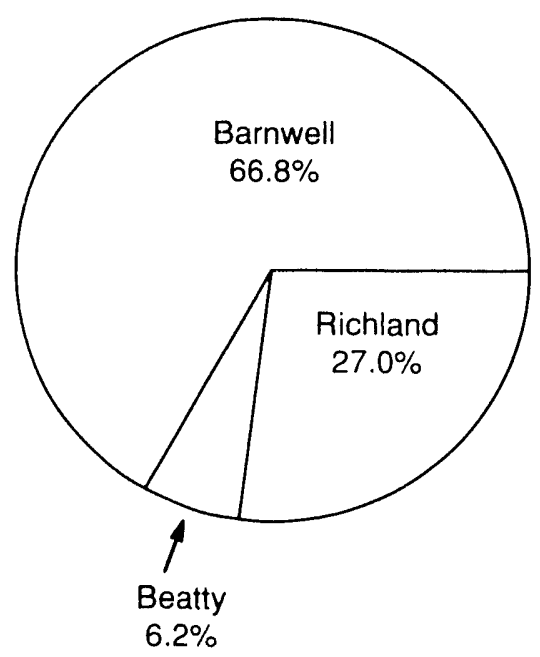

ACTIVITY PERCENTAGE BY DISPOSAL SITE (Percentages <. $1 \%$ are not displayed)

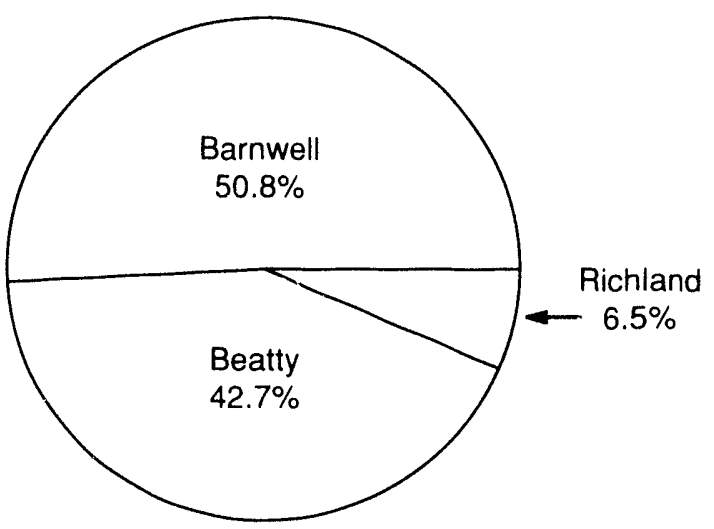

Volume Percentage by Waste Class for Direct Shipments and Total Non-Direct Volume Percentage (Percentages $<.1 \%$ are not displayed)

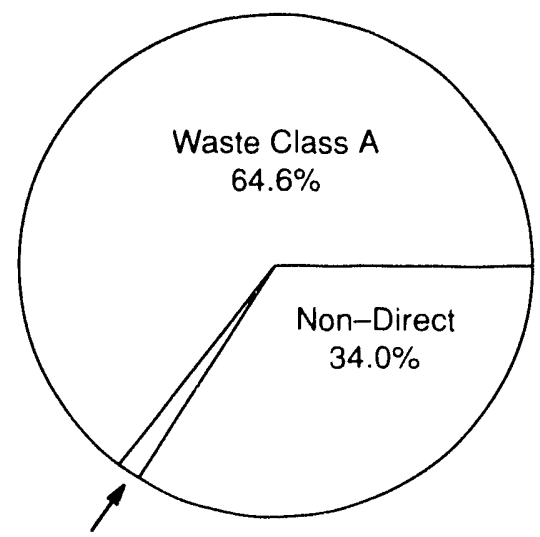

Waste Class B $1.4 \%$
Total Non-Direct Shipment

Volume

Direct Shipment

Waste Class

A

B

C

Total

Non-Direct Shipments

Grand Total

Total Direct Shipment

Activity (curies)

Grand Total (curies)
484.48

$6,657.69$

$19,609.59$

435.14

$12,665.70$

286.20

0.00

$12,951.90$

919.62 


\section{$\mathrm{OHIO}$ \\ Low-Level Radioactive Wastes Received at Commercial Disposal Sites}

\begin{tabular}{l} 
Generator \\
Category \\
\hline
\end{tabular}

Academic

Government

Industrial

Medical

Utility

Total
Volume

$\left(\mathrm{ft}^{3}\right)$

$1,028.00$

39.16

$2,016.35$

91.84

$20,971.50$

$24,146.85$
Activity

(curies)

61.47

88.72

14.22

1.33

$4,149.95$

$4,315.70$
VOLUME PERCENTAGE BY SOURCE

(Percentages <. $1 \%$ are not displayed)
ACTIVITY PERCENTAGE BY SOURCE

(Percentages <. $1 \%$ are not displayed)
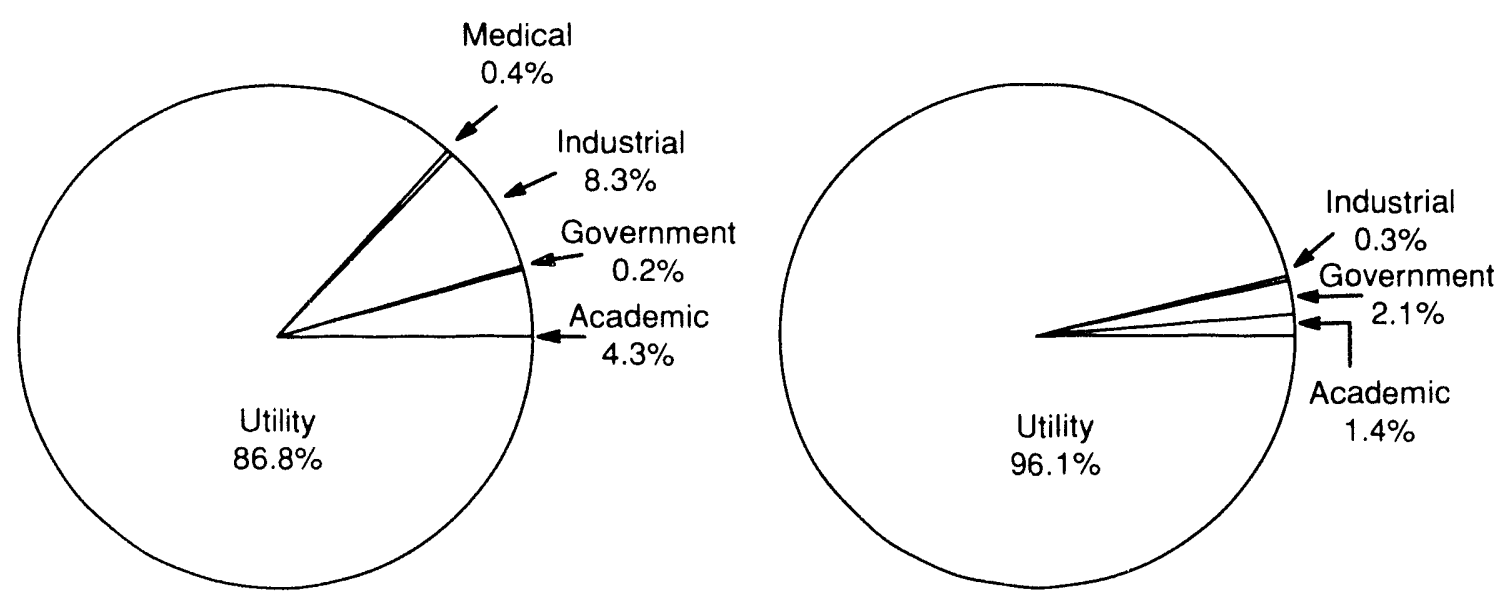


\section{$\mathrm{OHIO}$ \\ Low-Level Radioactive Wastes Received at Commercial Disposal Sites}

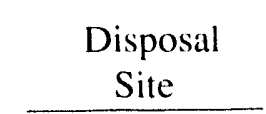

Barnwell

Beatty

Richland

Total
Volume
$\left(\mathrm{ft}^{3}\right)$

$11,530.75$

323.86

$12,292.24$

$24,146.85$
Activity

(curies)

$2,807.54$

139.42

$1,368.74$

$4,315.70$

\section{VOLUME PERCENTAGE BY DISPOSAL SITE (Percentages <. $1 \%$ are not displayed)}

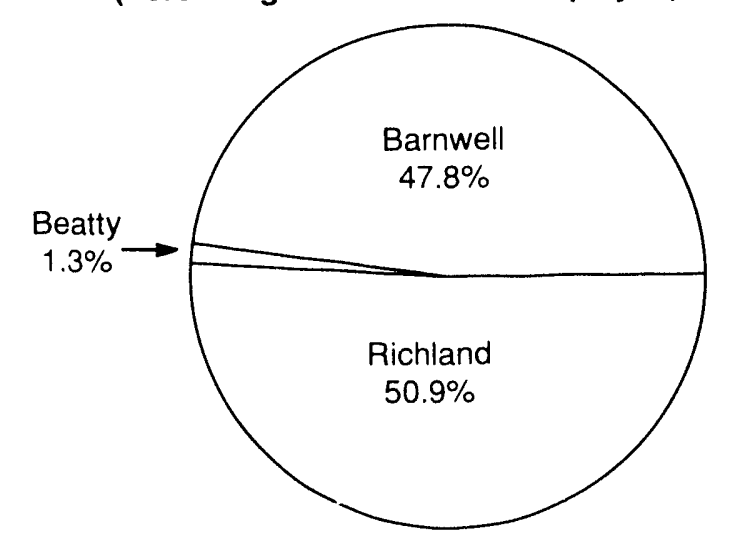

Direct Shipment Waste Class

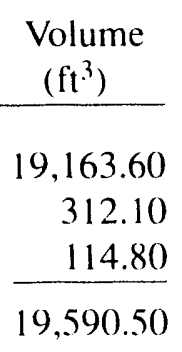
A
B
C

Total

Non-Direct Shipments

$4,556.35$

Grand Total

$24,146.85$

Total Direct Shipment

Activity (curies)

$4,146.37$

Total Non-Direct Shipment

Activity (curies)

169.33

Grand Total (curies)
ACTIVITY PERCENTAGE BY DISPOSAL SITE (Percentages <. $1 \%$ are not displayed)

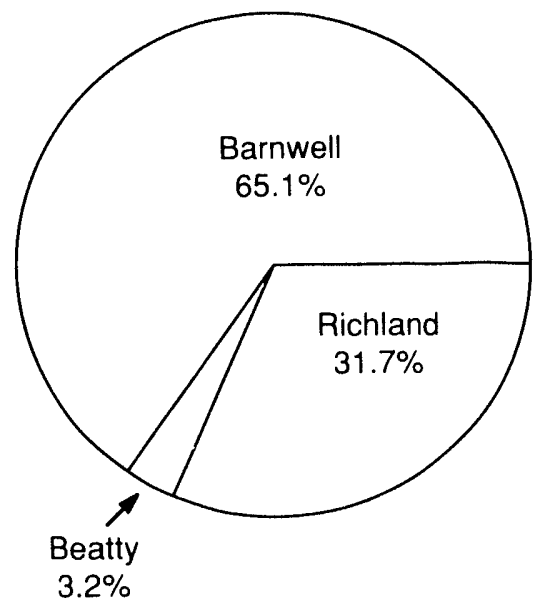

Volume Percentage by Waste Class for Direct Shipments and Total Non-Direct Volume Percentage (Percentages <. $1 \%$ are not displayed)

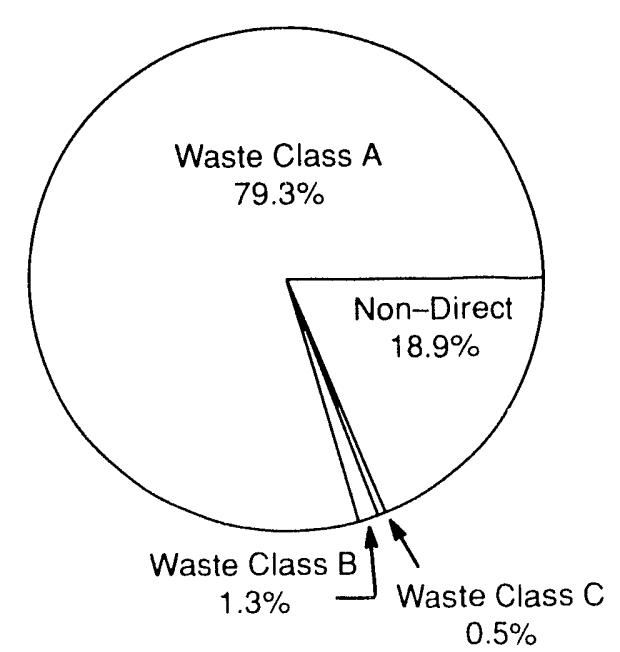




\section{WISCONSIN \\ Low-Level Radioactive Wastes Received at Commercial Disposal Sites}

\section{Generator \\ Category}

Academic

Government

Industrial

Medical

Utility

Total
Volume

$\left(\mathrm{ft}^{3}\right)$

129.74

4.01

347.30

50.99

$8,685.28$

$9,217.32$
Activity (curies)

$$
\begin{array}{r}
0.60 \\
<.01 \\
0.68 \\
0.51 \\
567.90 \\
\hline
\end{array}
$$

569.68

VOLUME PERCENTAGE BY SOURCE

ACTIVITY PERCENTAGE BY SOURCE

(Percentages <.1\% are not displayed)

(Percentages <. $1 \%$ are not displayed)
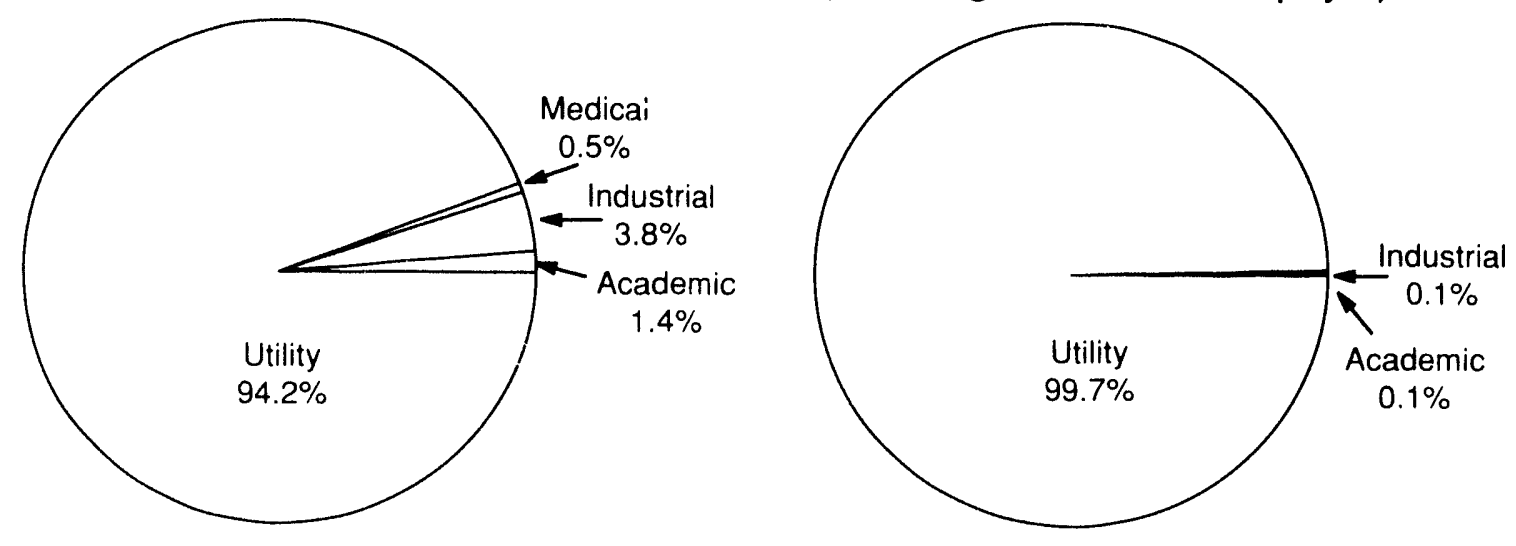


\section{WISCONSIN \\ Low-Level Radioactive Wastes Received at Commercial Disposal Sites}

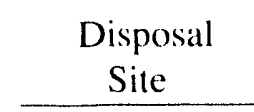

Barnwell

Beatty

Richland

Total

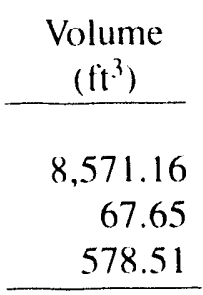

$9,217.32$

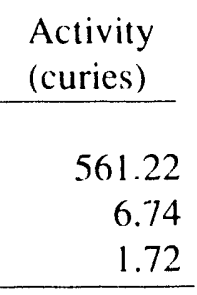

569.68

\section{VOLUME PERCENTAGE BY DISPOSAL SITE (Percentages $<.1 \%$ are not displayed)}

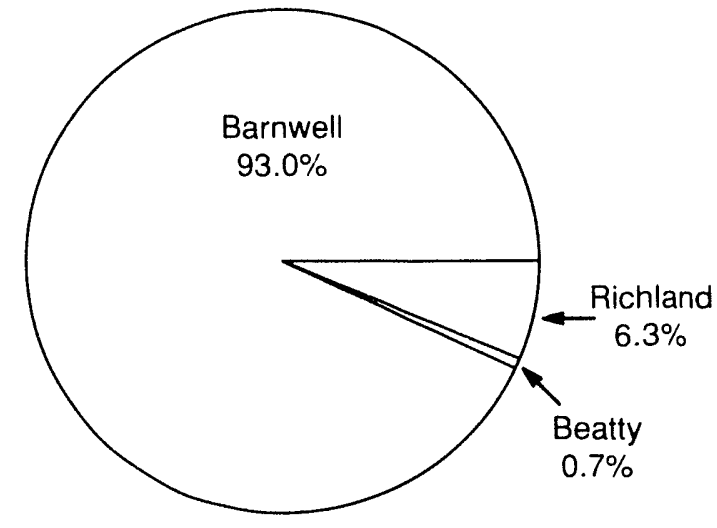

\section{ACTIVITY PERCENTAGE BY DISPOSAL SITE \\ (Percentages $<.1 \%$ are not displayed)}

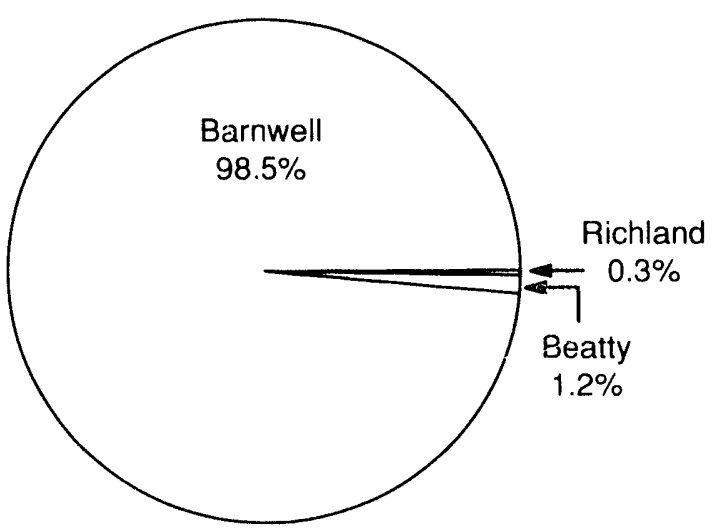

Volume Percentage by Waste Class for Direct Shipments and Total Non-Direct Volume Percentage (Percentages $<.1 \%$ are not displayed)

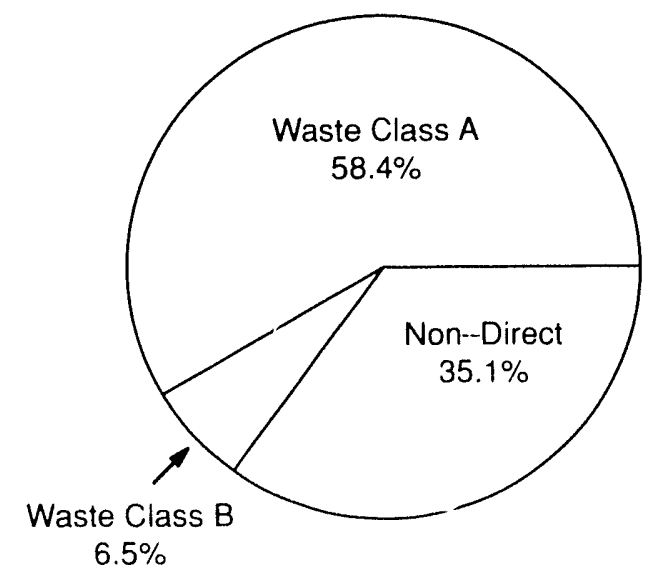

Direct Shipment

Waste Class

A

B

C

Total

Non-Direct Shipments

Grand Total

Total Direct Shiment

Activity (curies)

Total Non-Direct Shipment

Activity (curies)

Grand Total (curies)
Volume

$\left(\mathrm{ft}^{3}\right)$

$5,385.20$

599.50

0.00

$5,984.70)$

$3,232.62$

$9,217.32$

558.94

10.74

569.68 
】

】

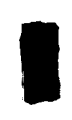

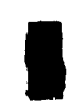

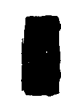

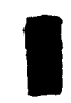

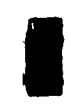

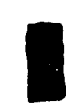

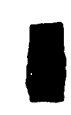

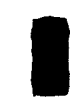

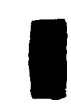

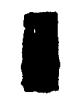

1

I

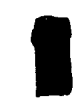

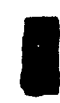

I

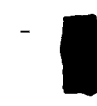

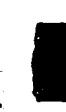




\section{NORTHEAST COMPACT \\ Low-Level Radioactive Wastes Received at Commerciai Disposal Sites}

\begin{tabular}{lcr}
\multicolumn{1}{c}{ States } & \multicolumn{1}{c}{$\begin{array}{c}\text { Volume } \\
\left(\mathrm{ft}^{3}\right)\end{array}$} & $\begin{array}{r}\text { Activity } \\
(\text { curies })\end{array}$ \\
Connecticut & $34,138.42$ & $255,037.79$ \\
New Jersey & $52,878.94$ & $3,717.50$ \\
Total & $87,017.36$ & 258.755 .29
\end{tabular}

VOLUME PERCENTAGE BY STATE

(Percentages <. $1 \%$ are not displayed)

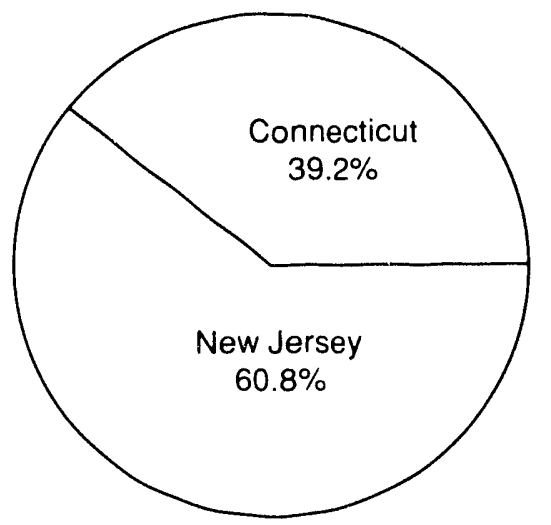

VOLUME PERCENTAGE BY CATEGORY

(Percentages <.1\% are not displayed)

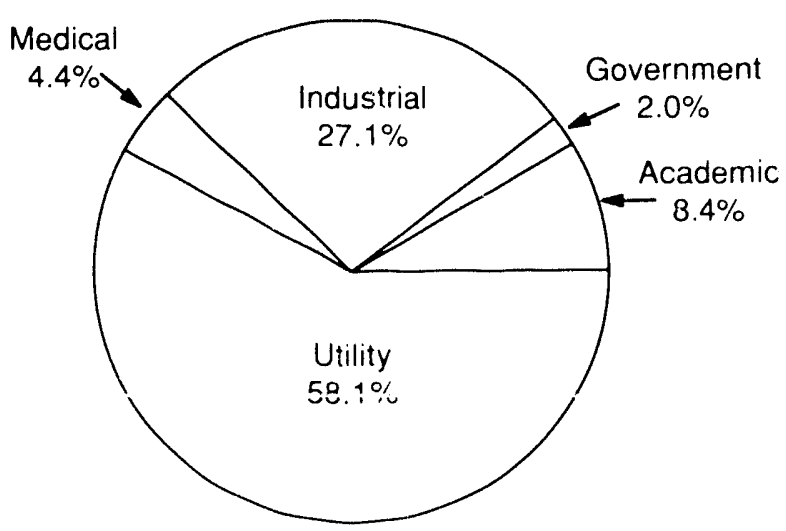




\section{CONNECTICUT \\ Low-Level Radioactive Wastes Received at Commercial Disposal Sites}

$\begin{array}{lrr}\begin{array}{l}\text { Generator } \\ \text { Category }\end{array} & \begin{array}{c}\text { Volume } \\ \left(\mathrm{ft}^{3}\right)\end{array} & \begin{array}{r}\text { A.ctivity } \\ \text { (curies) }\end{array} \\ \text { Academic } & 2,035.68 & 5.46 \\ \text { Government } & 1,716.70 & 0.51 \\ \text { Industrial } & 5,667.99 & 15.45 \\ \text { Medical } & 133.25 & 0.20 \\ \text { Utility } & 24,584.80 & 255,016.17 \\ \text { Total } & 34,138.42 & 255,037.79\end{array}$

VOLUME PERCENTAGE BY SOURCE (Percentages $<.1 \%$ are not displayed)

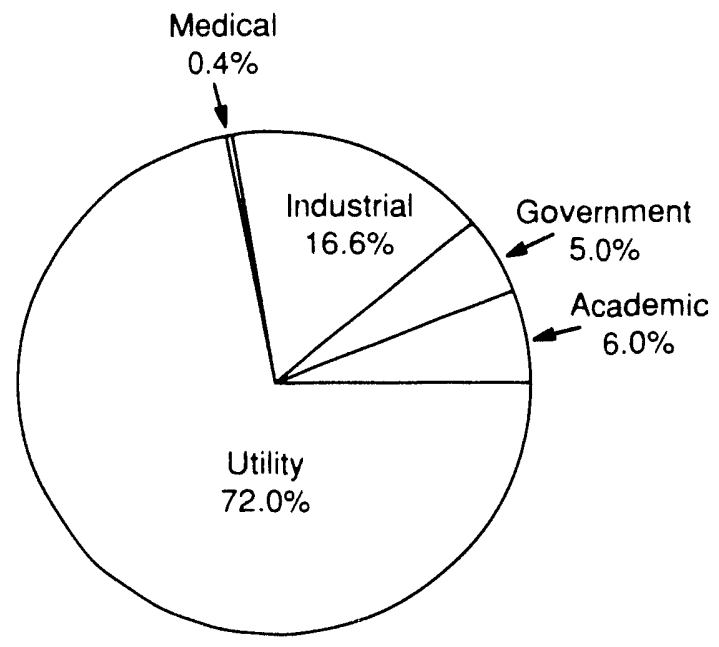

ACTIVITY PERCENTAGE BY SOURCE (Percentages $<.1 \%$ are not displayed)

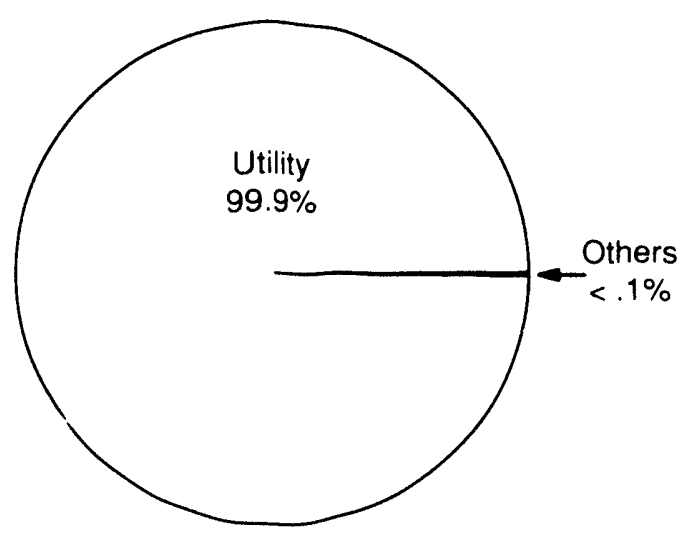




\section{CONNECTICUT \\ Low-Level Radioactive Wastes Received at Commercial Disposal Sites}

\begin{tabular}{c} 
Disposal \\
Site \\
\hline
\end{tabular}

Barnwell

Beatty

Richland

Total

\author{
Volume \\ $\left(\mathrm{ft}^{3}\right)$
}

$30,744.61$

36.60

$3,357.21$

$34,138.42$
Activity

(curies)

$255,023.51$

0.98

13.30

$255,037.79$

\section{VOLUME PERCENTAGE BY DISPOSAL SITE \\ (Percentages $<.1 \%$ are not displayed)}

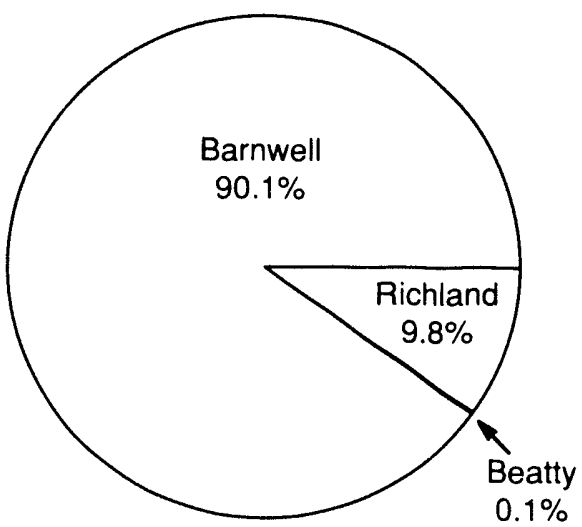

ACTIVITY PERCENTAGE BY DISPOSAL SITE

(Percentages $<.1 \%$ are not displayed)

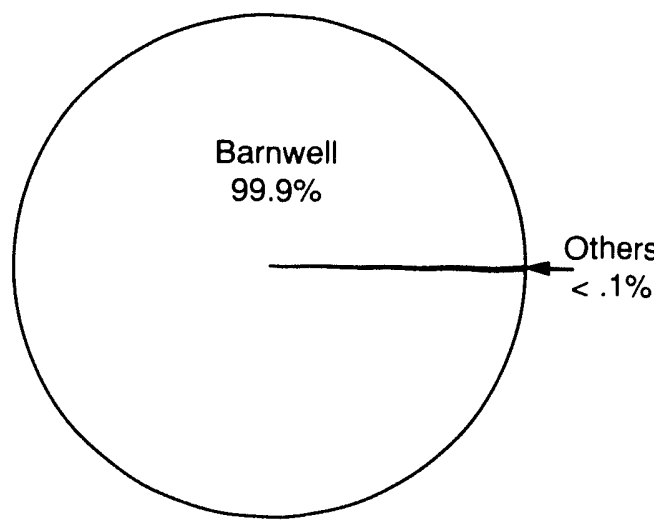

Volume Percentage by Waste Class
for Direct Shipments and Total
Non-Direct Volume Percentage
(Percentages <.1\% are not displayed)
Direct Shipment

Waste Class

A

B

C

Total

Non-Direct Shipments

Grand Total

Total Direct Shipment

Activity (curies)

Total Non-Direct Shipment

Activity (curies)

Grand Total (curies)
Volume $\left(\mathrm{ft}^{3}\right)$

$19,881.20$

$1,536.40$

$1,438.70$

$22,856.30$

$11,282.12$

$34,138.42$

$255,014.21$

23.58

$255,037.79$ 


\section{NEW JERSEY \\ Low-Level Radioactive Wastes Received at Commercial Disposal Sites}

\author{
Generator \\ Category \\ Academic \\ Government \\ Industrial \\ Medical \\ Utility
}

Total

$$
\begin{array}{r}
\begin{array}{c}
\text { Volume } \\
\left(\mathrm{ft}^{3}\right)
\end{array} \\
\\
5,311.65 \\
2.00 \\
17,887.74 \\
3,677.06 \\
26,000.49 \\
\hline
\end{array}
$$

$52,878.94$
Activity

(curies)

5.13

5.42

151.46

4.04

$3,551.44$

$3,717.50$

VOLUME PERCENTAGE BY SOURCE

ACTIVITY PERCENTAGE BY SOURCE

(Percentages <. $1 \%$ are not displayed)

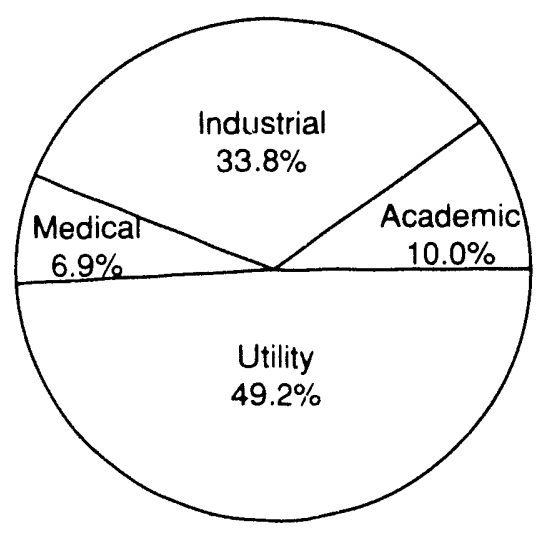

(Percentages <. $1 \%$ are not displayed)

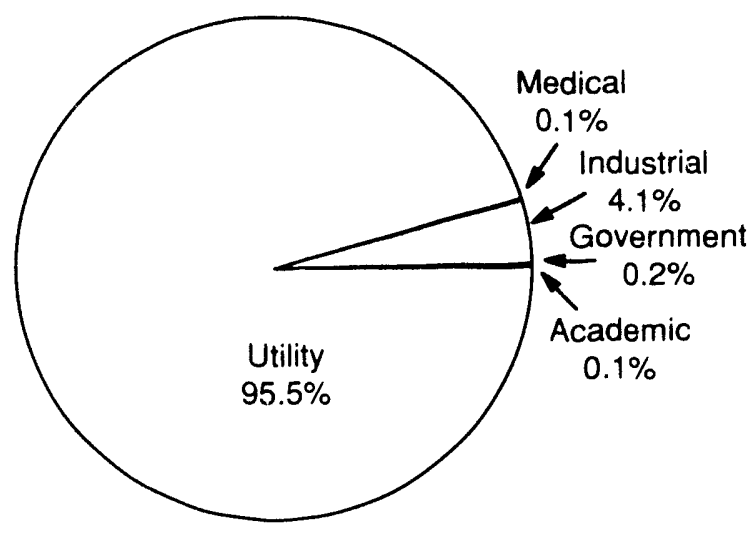




\section{NEW JERSEY \\ Low-Level Radioactive Wastes Received at Commercial Disposal Sites}

\begin{tabular}{c} 
Disposal \\
Site \\
\hline
\end{tabular}

Barnwell

Beatty

Richland

Total

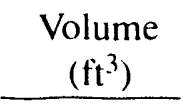

$42,175.41$

$1,349.52$

$9,354.01$

$52,878.94$
Activity

(curies)

$3,638.51$

3.15

75.84

$3,717.50$
VOLUME PERCENTAGE BY DISPOSAL SITE

(Percentages <. $1 \%$ are not displayed)

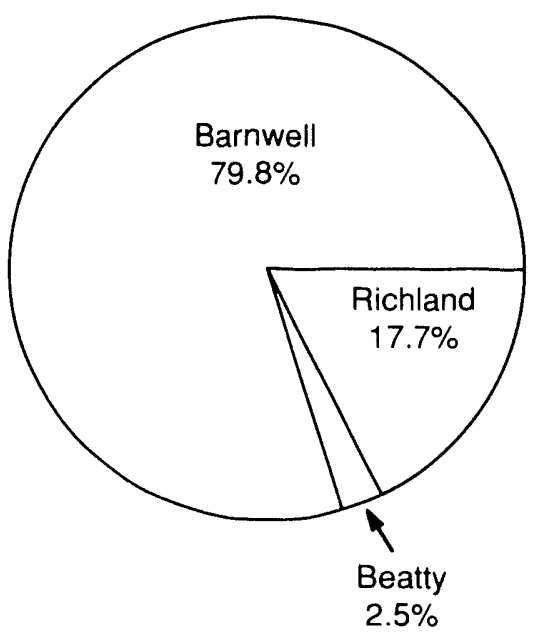

Direct Shipment

Waste Class

A

B

C

Total

Non-Direct Shipments

Grand Total

Total Direct Shipment

Activity (curies)

Total Non-Direct Shipment

Activity (curies)

Grand Total (curies) $\left(\mathrm{ft}^{3}\right)$
ACTIVITY PERCENTAGE BY DISPOSAL SITE

(Percentages <.1\% are not displayed)

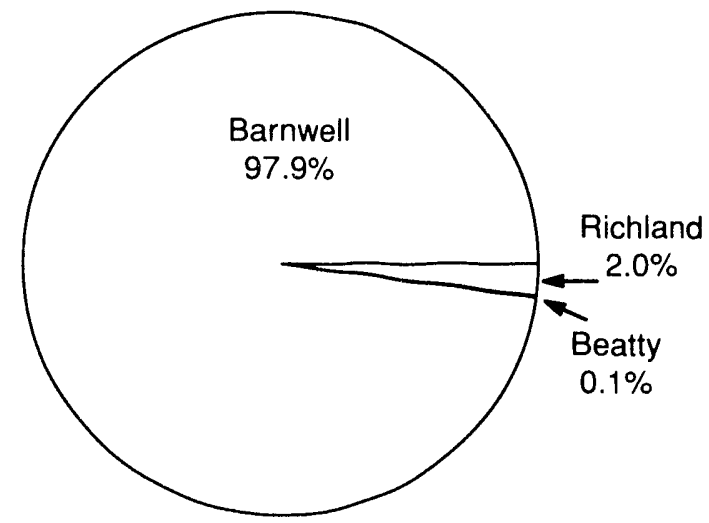

Volume Percentage by Waste Class for Direct Shipments and Total Non-Direct Voluine Percentage (Percentages <.1\% are not displayed)

Volume

$21,331.98$

$1,938.60$

191.10

$23,461.68$

$29,417.26$

$52,878.94$

3.534 .25

183.24

$3.71 \% .50$

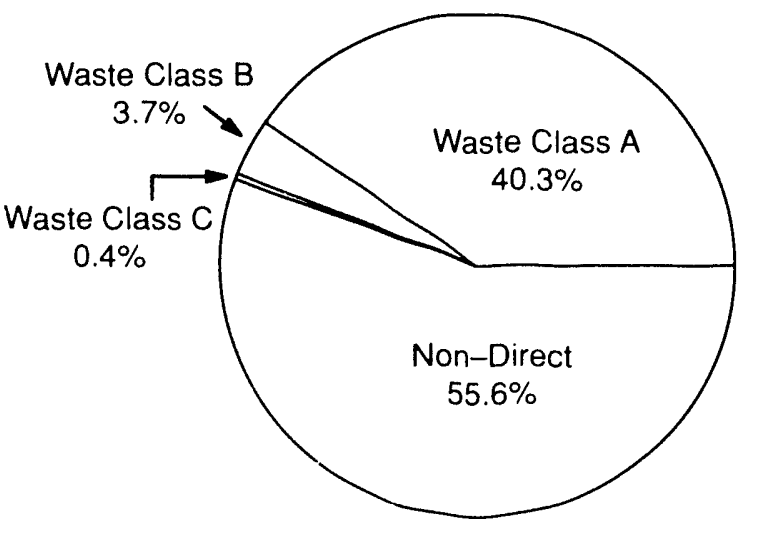




\section{NORTHWEST COMPACT \\ Low-Level Radioactive Wastes Received at Commercial Disposal Sites}

\begin{tabular}{l}
$-\frac{1}{\quad \text { States }}$ \\
\hline Alaska \\
Hawaii \\
Idaho \\
Montana \\
Oregon \\
Utah \\
Washington \\
Total
\end{tabular}

$\begin{array}{r}\multicolumn{1}{c}{\begin{array}{c}\text { Volume } \\ \left(\mathrm{ft}^{3}\right)\end{array}} \\ \hline 33.51 \\ 4,738.86 \\ 40.06 \\ 195.00 \\ 59,456.32 \\ 5,338.12 \\ 26,141.09 \\ \hline 95,942.96\end{array}$

Activity

(curies)

225.46

0.68

0.42

0.07

592.90

1.40

$1,397.77$

$2,218.70$

VOLUME PERCENTAGE BY STATE

(Percentages $<.1 \%$ are not displayed)

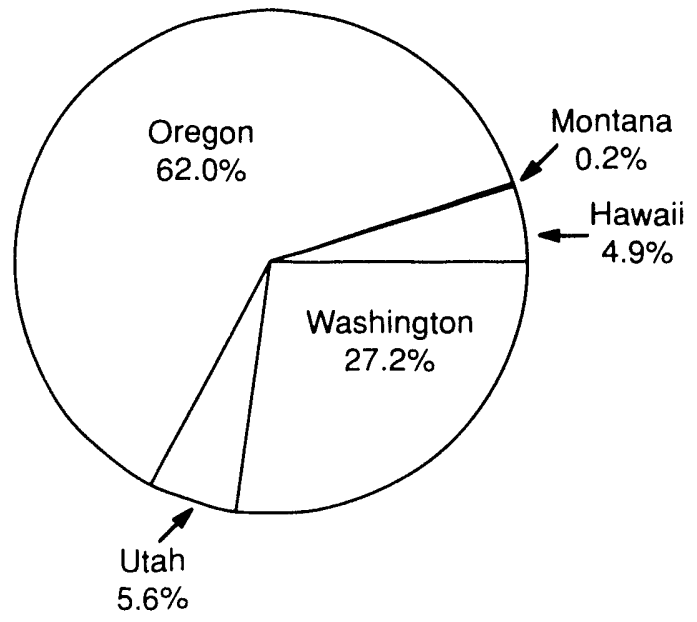

VOLUME PERCENTAGE BY CATEGORY

(Percentages <. $1 \%$ are not displayed)

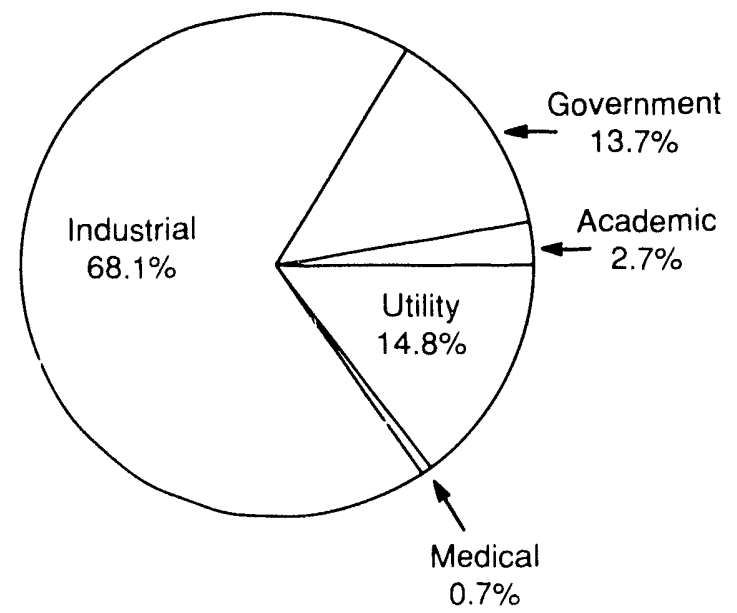




\section{ALASKA \\ Low-Level Radioactive Wastes Received at Commercial Disposal Sites}

\section{Generator}

Category

Government

Industrial

Total

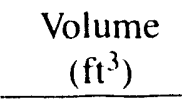

3.51

30.00

33.51
Activity

(curies)

3.46

222.00

225.46
VOLUME PERCENTAGE BY SOURCE

(Percentages <. $1 \%$ are not displayed)

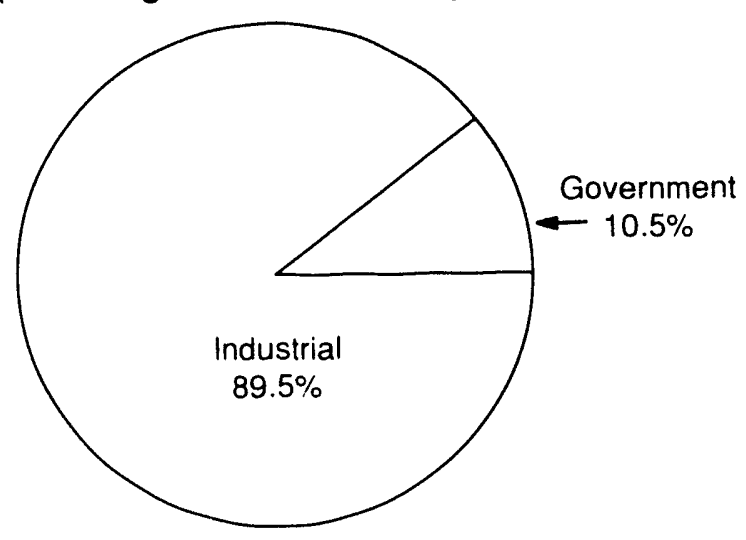

ACTIVITY PERCENTAGE BY SOURCE

(Percentages $<.1 \%$ are not displayed)

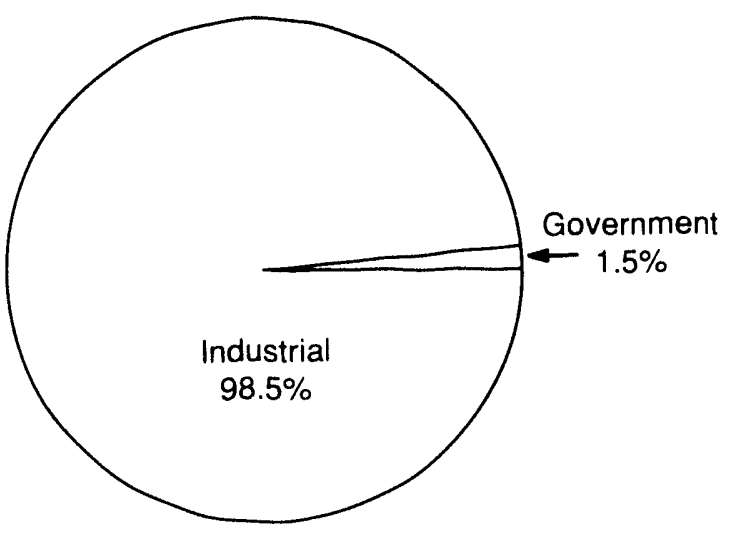




\section{ALASKA \\ Low-Level Radioactive Wastes Received at Commercial Disposal Sites}

\begin{tabular}{c} 
Disposal \\
Site \\
\hline
\end{tabular}

Beatty

Richland

Total

\section{Volume}

$\left(\mathrm{ft}^{3}\right)$

$$
30.00
$$

3.51

33.51
Activity

(curies)

222.00

3.46

225.46
VOLUME PERCENTAGE BY DISPOSAL SITE (Percentages <. $1 \%$ are not displayed)

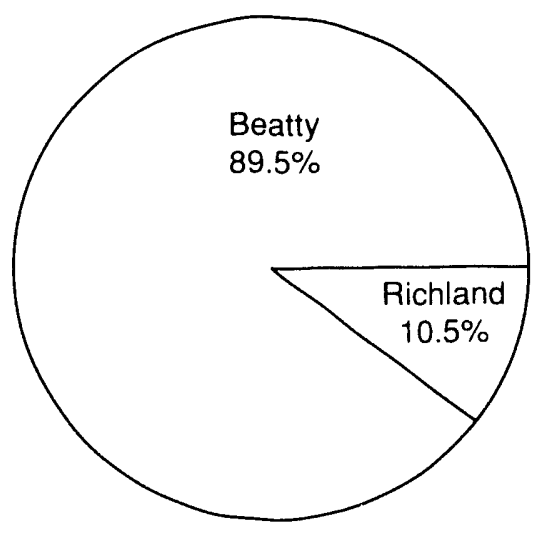

ACTIVITY PERCENTAGE BY DISPOSAL SITE

(Percentages $<.1 \%$ are not displayed)

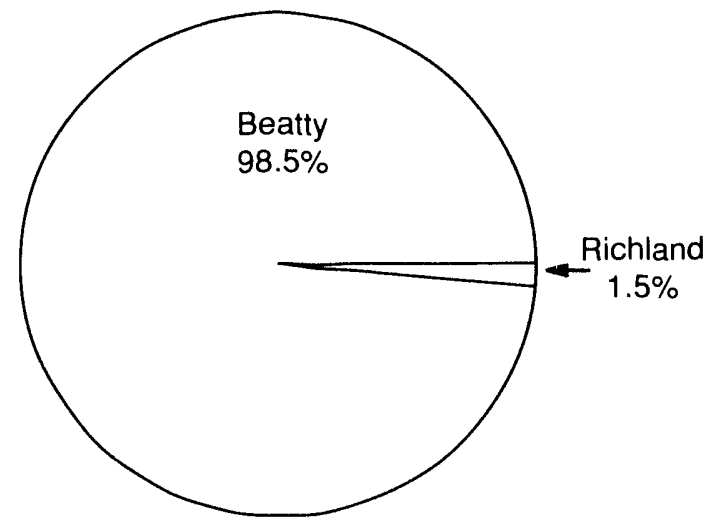

Volume Percentage by Waste Class for Direct Shipments and Total

Non-Direct Volume Percentage

(Percentages <. $1 \%$ are not displayed)

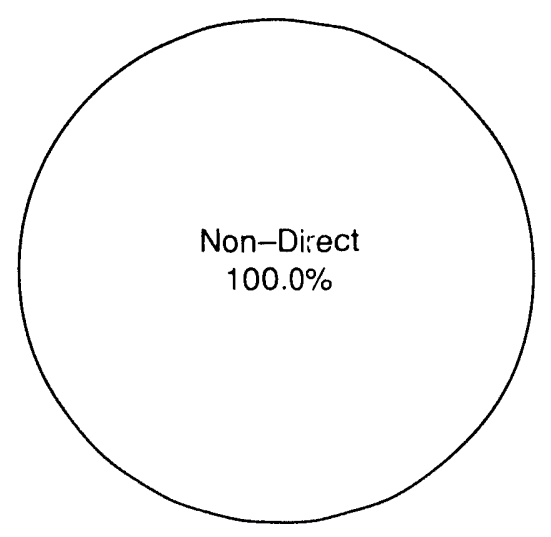




\section{HAWAII \\ Low-Level Radioactive Wastes Received at Commercial Disposal Sites}

\section{Generator \\ Category}

Academic

Government

Total

\begin{tabular}{c}
$\begin{array}{c}\text { Volume } \\
\left(\mathrm{ft}^{3}\right)\end{array}$ \\
\hline
\end{tabular}

14.07

$4,724.79$

$4,738.86$
Activity

(curies)

0.17

0.51

0.68
VOLUME PERCENTAGE BY SOURCE

(Percentages $<.1 \%$ are not displayed)

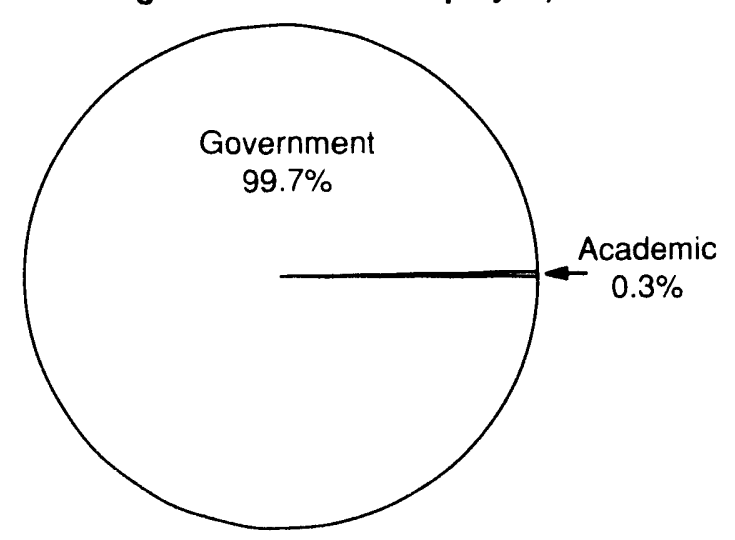

ACTIVITY PERCENTAGE BY SOURCE (Percentages <. $1 \%$ are not displayed)

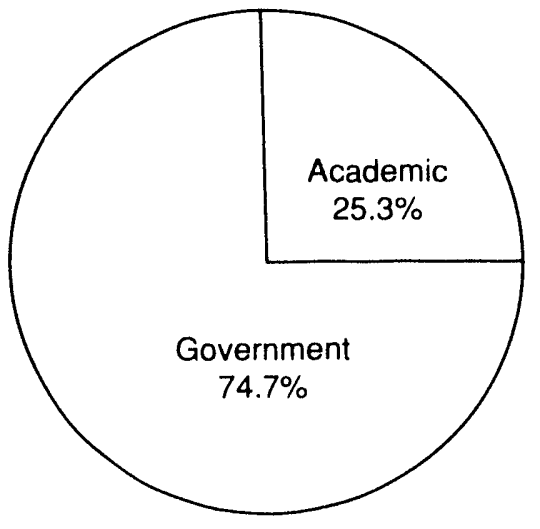




\section{HAWAII \\ Low-Level Radioactive Wastes Received at Commercial Disposal Sites}

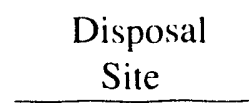

Richland

Total

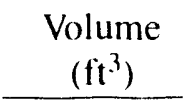

$4,738.86$

$4,738.86$
Activity

(curies)

0.68

0.68
VOLUME PERCENTAGE BY DISPOSAL SITE

(Percentages $<.1 \%$ are not displayed)

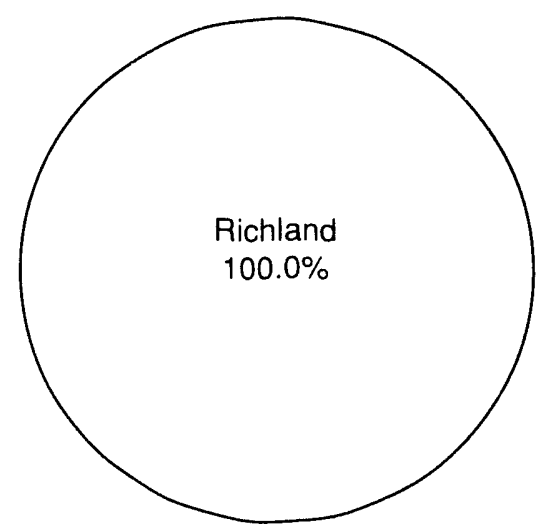

Direct Shipment

Waste Class

Volume

$\left(\mathrm{ft}^{3}\right)$

A

B

C

Total

Non-Direct Shipments

GrandTotal

Total Direct Shipment

Activity (curies)

Total Non-Direct Shipment

Activity (curies)

Grand Total (curies)
$4,724.79$

0.00

0.00

$4,724.79$

14.07

$4,738.86$

0.51

0.17

0.68
ACTIVITY PERCENTAGE BY DISPOSAL SITE

(Percentages <. $1 \%$ are not displayed)

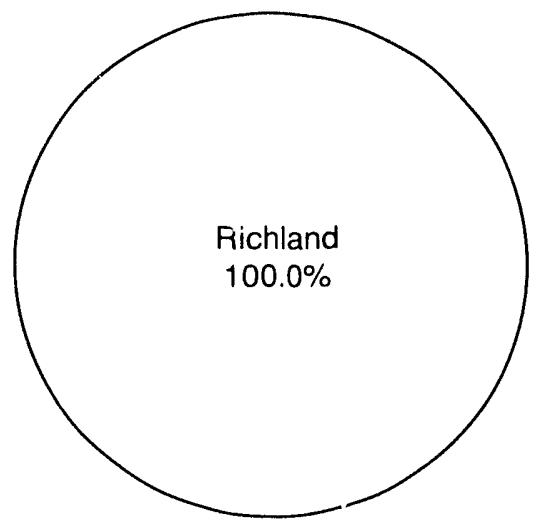

Volume Percentage by Waste Class

for Direct Shipments and Total

Non-Direct Volume Percentage

(Percentages <. $1 \%$ are not displayed)

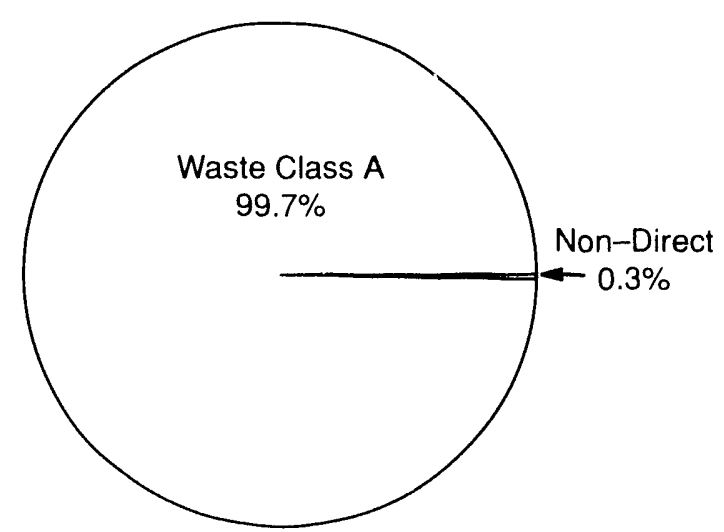




\section{IDAHO \\ Low-Level Radioactive Wastes Received at Commercial Disposal Sites}

\begin{tabular}{l} 
Generator \\
Category \\
\hline
\end{tabular}

Acartemic

Industrial

Medical

Total

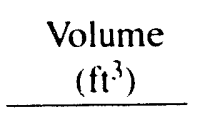

30.60

9.39

0.07

40.06
Activity

(curies)

0.07

0.35

$<.01$

0.42
VOLUME PERCENTAGE BY SOURCE (Percentages <. $1 \%$ are not displayed)

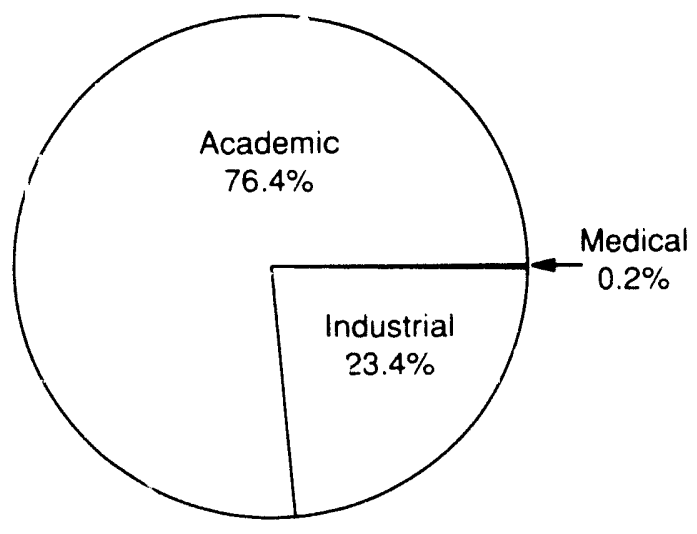

ACTIVITY PERCENTAGE BY SOURCE

(Percentages <. $1 \%$ are not displayed)

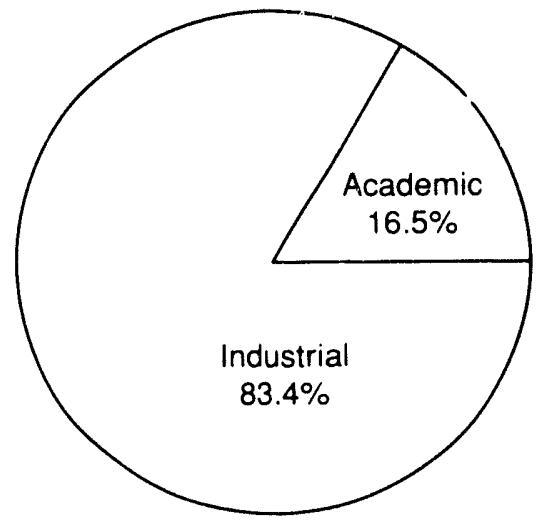




\section{IDAHO \\ Low-Level Radioactive Wastes Received at Commercial Disposal Sites}

\begin{tabular}{lrr}
\multicolumn{1}{c}{$\begin{array}{c}\text { Disposal } \\
\text { Site }\end{array}$} & $\begin{array}{c}\text { Volume } \\
\left(\mathrm{ft}^{3}\right)\end{array}$ & \multicolumn{1}{c}{$\begin{array}{c}\text { Activity } \\
\text { (curies) }\end{array}$} \\
$\begin{array}{l}\text { Beatty } \\
\text { Richland }\end{array}$ & $\begin{array}{r}9.46 \\
30.60\end{array}$ & 0.35 \\
Total & 40.06 & 0.07 \\
\cline { 2 - 3 } & & 0.42
\end{tabular}

VOLUME PERCENTAGE BY DISPOSAL SITE

(Percentages <. $1 \%$ are not displayed)

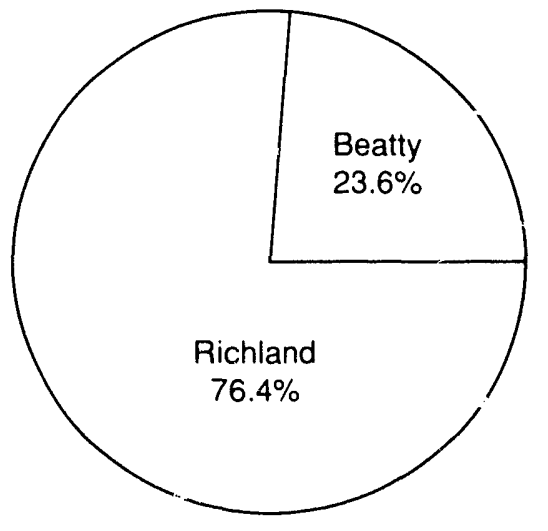

ACTIVITY PERCENTAGE BY DISPOSAL SITE

(Percentages $<.1 \%$ are not displayed)

Volume Percentage by Waste Class

for Direct Shipments and Total

Non-Direct Volume Percentage

1

(Percentages <. $\%$ are not displayed)

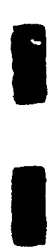
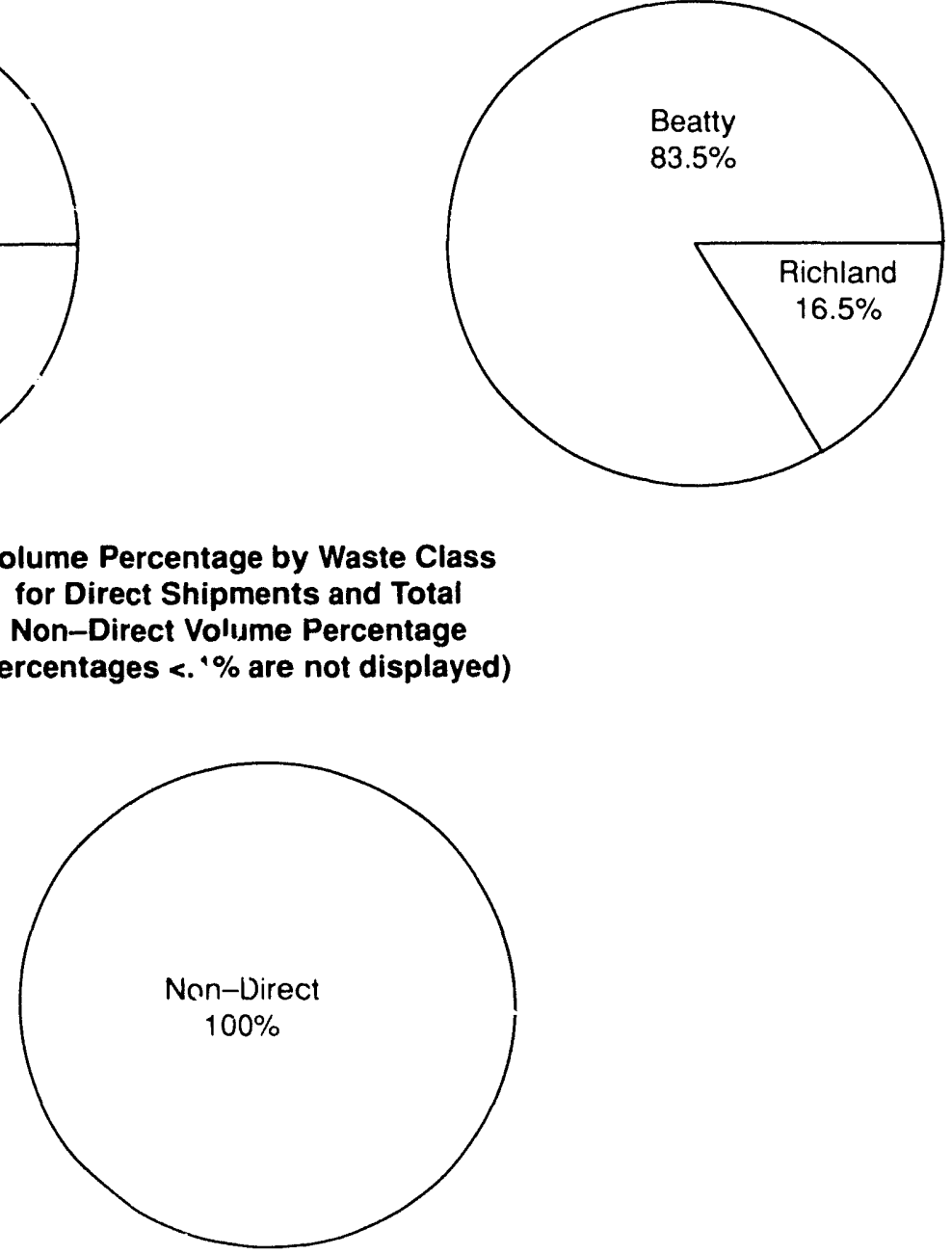


\section{MONTANA \\ Low-Level Radioactive Wastes Received at Commercial Disposal Sites}

\begin{tabular}{l} 
Generator \\
Category \\
\hline
\end{tabular}

Academic

Total

\begin{tabular}{c}
$\begin{array}{c}\text { Volume } \\
\left(\mathrm{ft}^{3}\right)\end{array}$ \\
\hline
\end{tabular}

195.00

195.00
Activity

(curies)

0.07

0.07

VOLUME PERCENTAGE BY SOURCE

(Percentages <. $1 \%$ are not displayed)

ACTIVITY PERCENTAGE BY SOURCE

(Percentages <. $1 \%$ are not displayed)
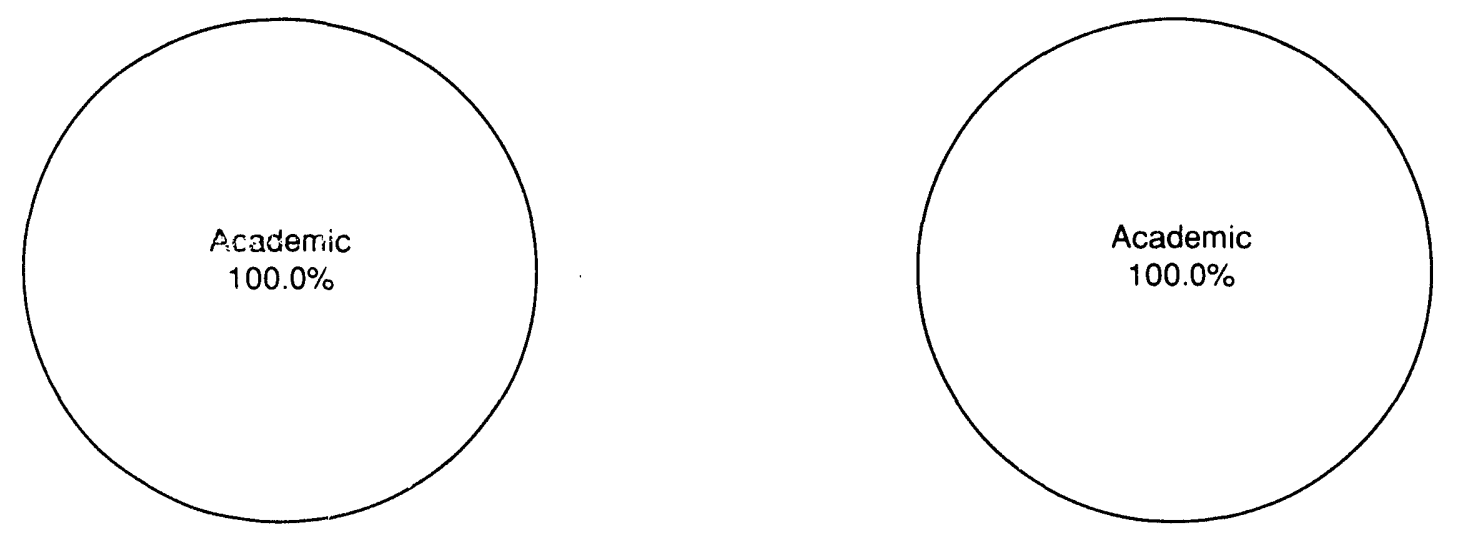


\section{MONTANA \\ Low-Level Radioactive Wastes Received at Commercial Disposal Sites}

Disposal
Site

Richland

Total

Volume
$\left(\mathrm{ft}^{3}\right)$

195.00

195.00
Activity

(curies)

0.07

0.07
VOLUME PERCENTAGE BY DISPOSAL SITE

(Percentages <.1\% are not displayed)

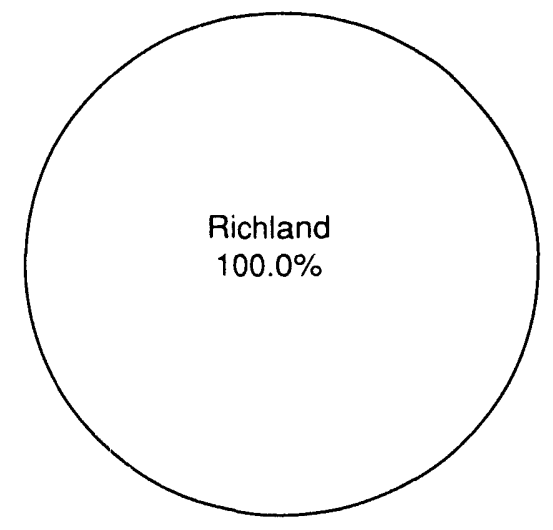

ACTIVITY PERCENTAGE BY DISPOSAL SITE (Percentages <. $1 \%$ are not displayed)

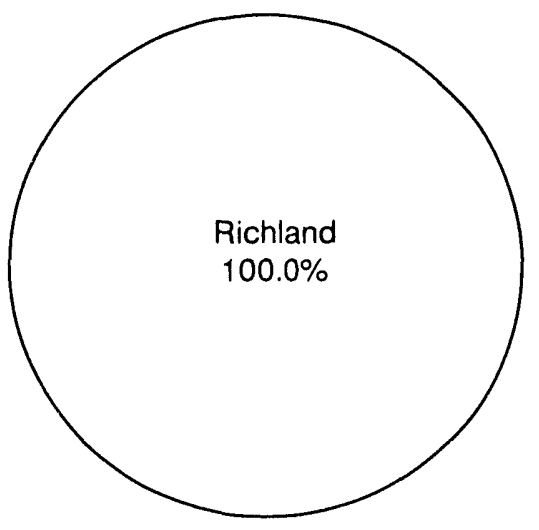

Volume Percentage by Waste Class for Direct Shipments and Total Non-Direct Volume Percentage (Percentages <. $1 \%$ are not displayed)
Direct Shipment

Waste Class
A
B
C

Total

Volume $\left(\mathrm{ft}^{3}\right)$

150.00

0.00

0.00

150.00

Non-Direct Shipments

45.00

Grand Total

195.00

Total Direct Shipment

Activity (curies)

0.05

Tota! Non-Direct Shipmen!

Activity (curies)

0.02

Grand Total (curies)
0.07

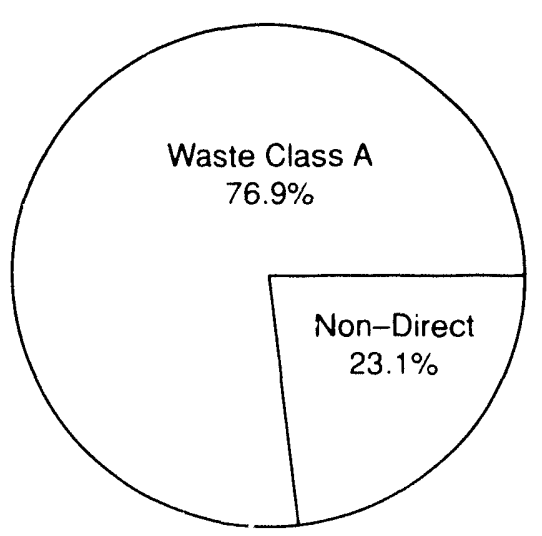




\section{OREGON \\ Low-Level Radioactive Wastes Received at Commercial Disposal Sites}

\begin{tabular}{l} 
Generator \\
Category \\
\hline
\end{tabular}

Academic

Industrial

Medical

Utility

Total

\begin{tabular}{c}
$\begin{array}{c}\text { Volume } \\
\left(\mathrm{ft}^{3}\right)\end{array}$ \\
\hline
\end{tabular}

316.86

$56,728.31$

7.05

$2,404.10$

$59,456.32$
Activity (curies)

$\begin{array}{r}1.90 \\ 5.50 \\ 0.05 \\ 585.45 \\ \hline\end{array}$

VOLUME PERCENTAGE BY SOURCE (Percentages <. $1 \%$ are not displayed)

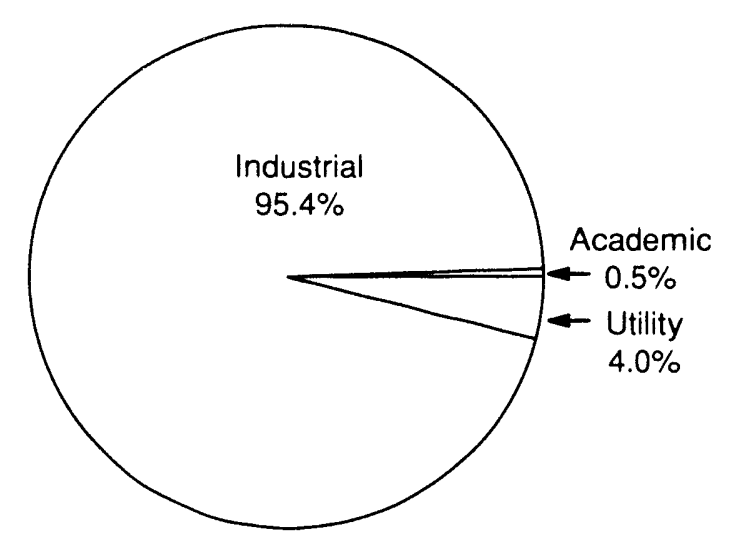

ACTIVITY PERCENTAGE BY SOURCE

(Percentages <. $1 \%$ are not displayed)

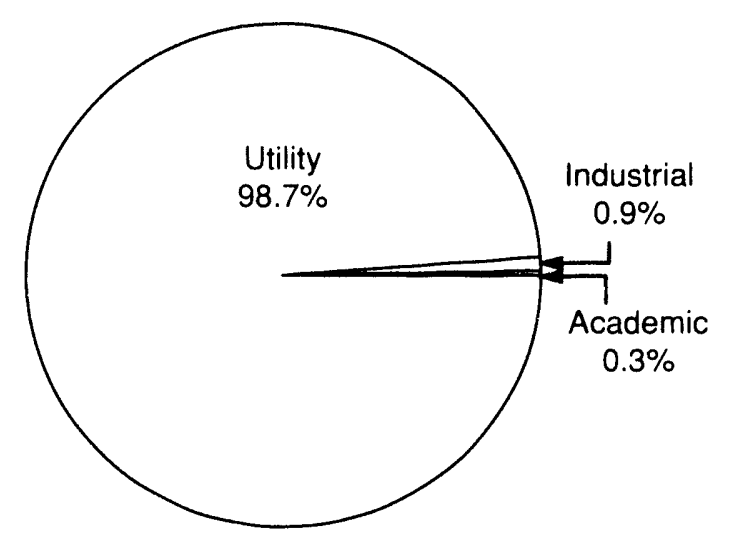




\section{OREGON \\ Low-Level Radioactive Wastes Received at Commercial Disposal Sites}

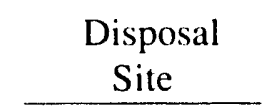

Beatty

Richland

Total

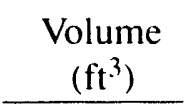

15.07

$59,441.25$

$59,456.32$
Activity

(curies)

0.64

592.26

592.90
VOLUME PERCENTAGE BY DISPOSAL SITE (Percentages <. $1 \%$ are not displayed)

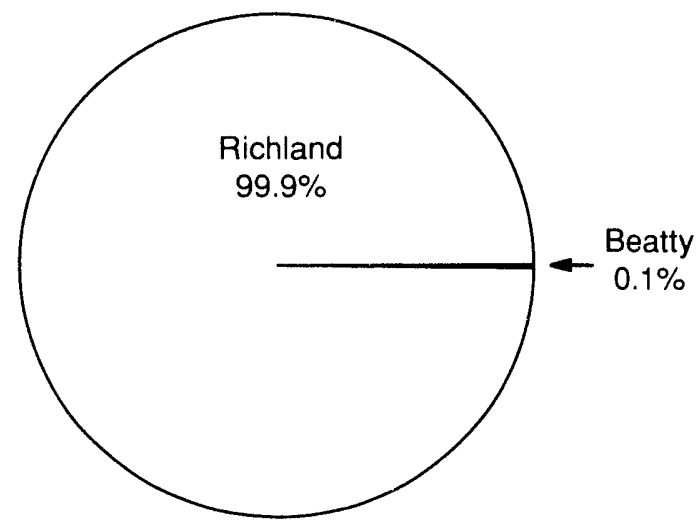

ACTIVITY PERCENTAGE BY DISPOSAL SITE (Percentages <. $1 \%$ are not displayed)

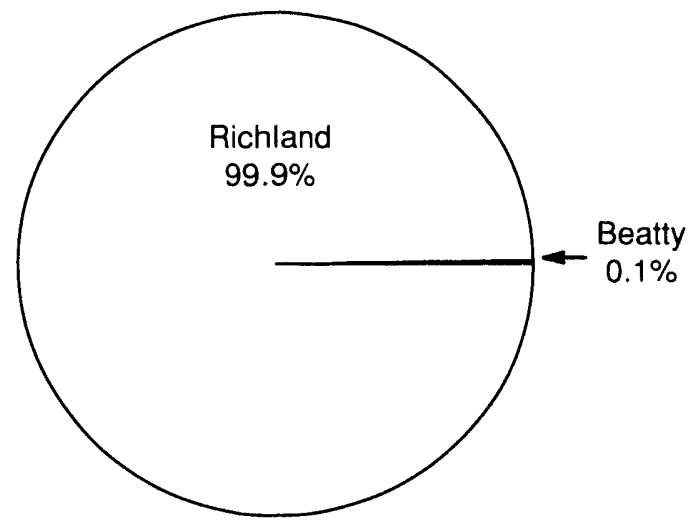

Volume Percentage by Waste Class for Direct Shipments and Total Non-Direct Volume Percentage (Percentages $<.1 \%$ are not displayed)

Direct Shipment Waste Class \begin{tabular}{c}
$\begin{array}{c}\text { Volume } \\
\left(\mathrm{ft}^{3}\right)\end{array}$ \\
\hline
\end{tabular} A

B

$56,609.40$

132.00

132.00

C

Total

$56,873.40$

Non-Direct Shipments

2.582 .92

Grand Total

$59,4.56 .32$

Total Direct Shipment Activity (curies)

586.64

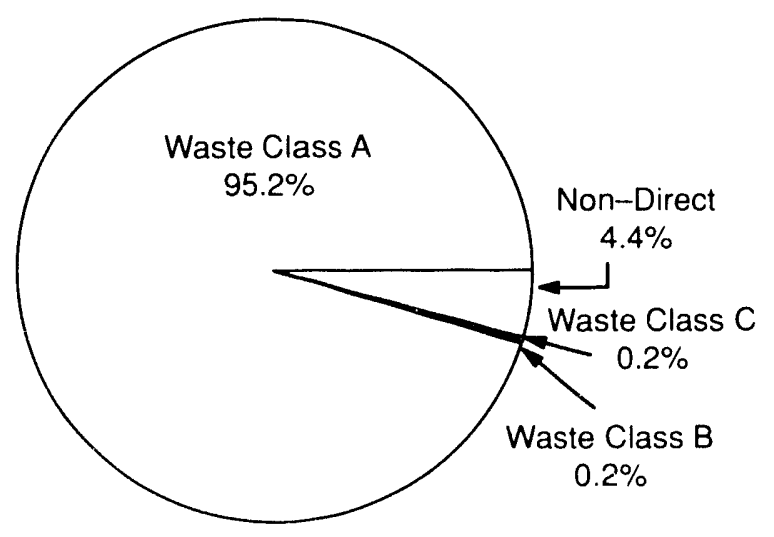

Total Non-Direct Shipment

Activity (curies)

6.20

Grand Total (curies)

592.90 


\section{UTAH \\ Low-Level Radioactive Wastes Received at Commercial Disposal Sites}

\section{Generator \\ Category}

Academic

Industrial

Medical

Total

\author{
Volume \\ $\left(\mathrm{ft}^{3}\right)$ \\ 753.86 \\ $4,571.13$ \\ 13.13
}

$5,338.12$
Activity

(curies)

0.91

0.36

0.13

1.40
VOLUME PERCENTAGE BY SOURCE

(Percentages $<.1 \%$ are not displayed)

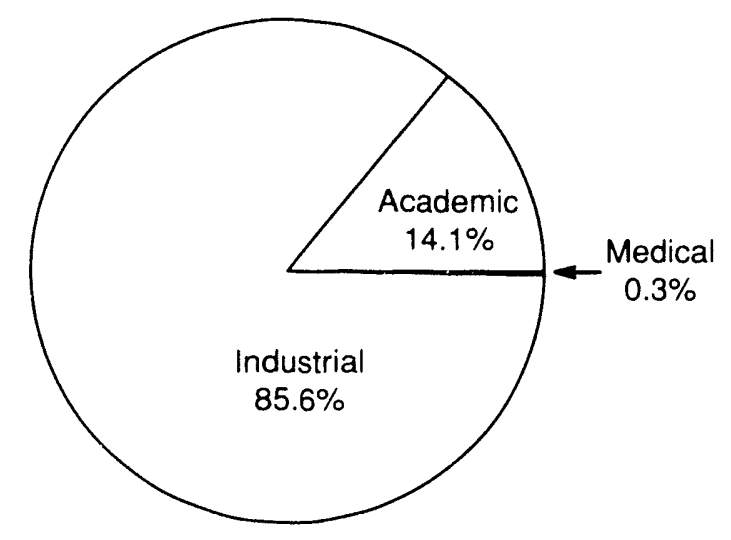

ACTIVITY PERCENTAGE BY SOURCE

(Percentages $<.1 \%$ are not displayed)

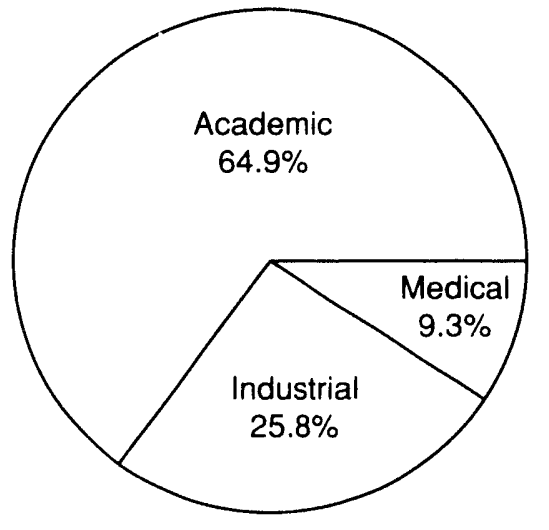




\section{UTAH \\ Low-Level Radioactive Wastes Received at Commercial Disposal Sites}

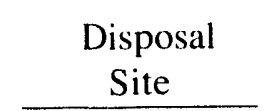

Beatty

Richland

Total

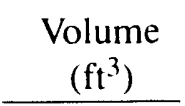

22.35

$5,315.77$

$5,338.12$
Activity

(curies)

0.25

1.14

1.39
VOLUME PERCENTAGE BY DISPOSAL SITE (Percentages <. $1 \%$ are not displayed)

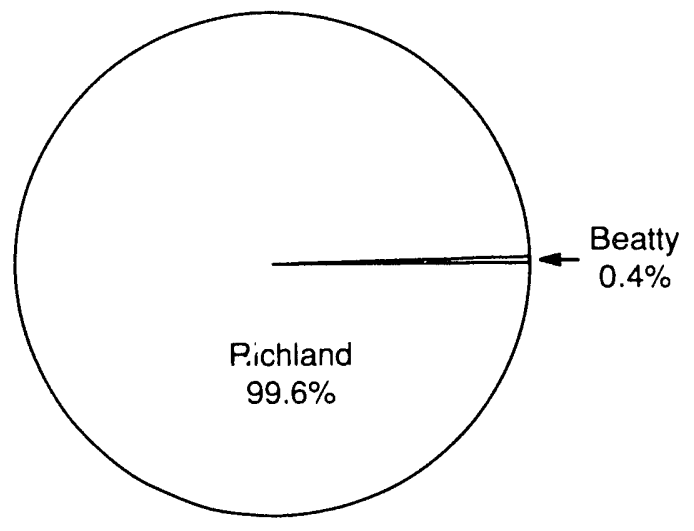

ACTIVITY PERCENTAGE BY DISPOSAL SITE

(Percentages $<.1 \%$ are not displayed)

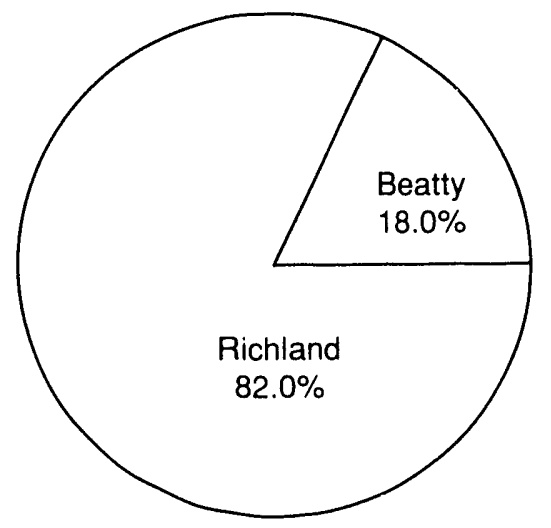

\section{Volume Percentage by Waste Class for Direct Shipments and Total Non-Direct Volume Percentage (Percentages <.1\% are not displayed)}

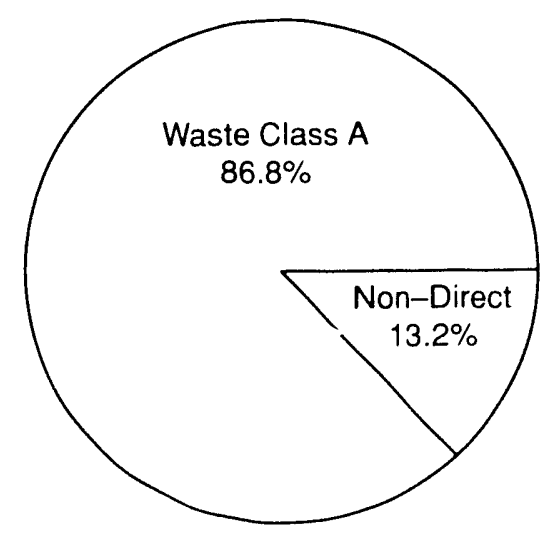

Direct Shipment

Waste Class

A

B

C

Total

Non-Direct Shipments

GrandTotal

Total Direct Shipment

Activity (curie ;)

Total Non-Direct Shipment

Activity (curies)

Grand Total (curies)
Volume

$\left(\mathrm{ft}^{3}\right)$

$4,633.00$

0.00

0.00

$4,633.00$

705.12

$5,338.12$

0.24

1.15

1.39 


\section{WASHINGTON \\ Low-Level Radioactive Wastes Received at Commercial Disposal Sites}

\author{
Generator \\ Category \\ Academic \\ Government \\ Industrial \\ Medical \\ Utility
}

Total

Volume
$\left(\mathrm{ft}^{3}\right)$

$1,316.13$

$8,445.50$

$3,951.15$

641.51

$11,786.80$

$26,141.09$
Activity

(crries)

$$
4.92
$$

92.41

9.92

0.11

$1,290.40$

$1,397.77$
VOLUME PERCENTAGE BY SOURCE (Percentages <. $1 \%$ are not displayed)

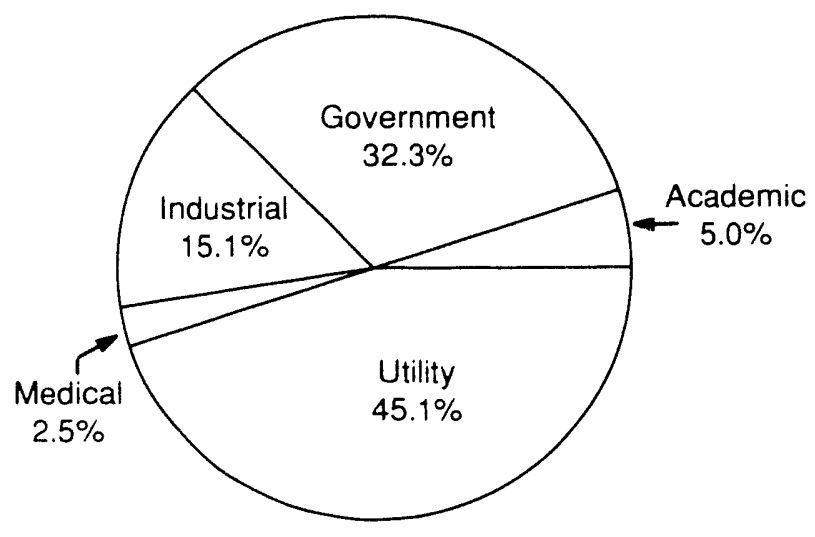

ACTIVITY PERCENTAGE BY SOURCE

(Percentages $<.1 \%$ are not displayed)

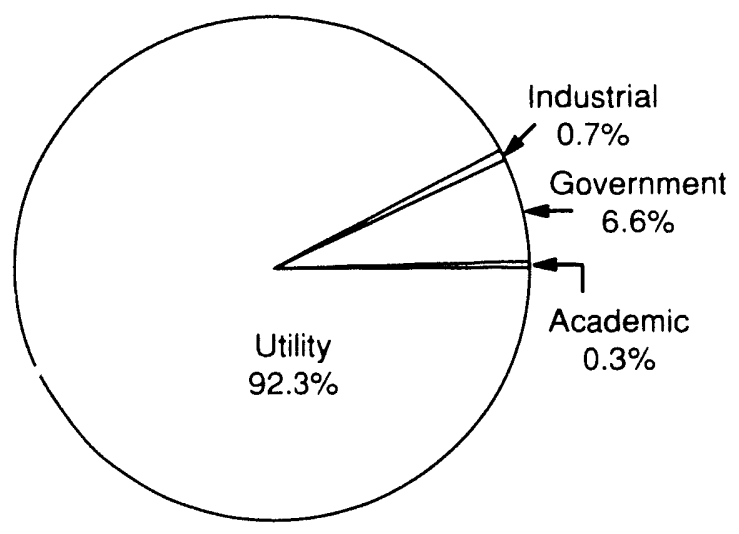




\section{WASHINGTON \\ Low-Level Radioactive Wastes Received at Commercial Disposal Sites}

\begin{tabular}{c} 
Disposal \\
Site \\
\hline
\end{tabular}

Beatty

Richland

Total

\begin{tabular}{c}
$\begin{array}{c}\text { Volume } \\
\left(\mathrm{ft}^{3}\right)\end{array}$ \\
\hline
\end{tabular}

16.15

$26,124.94$

$26,141.09$
Activity

(curies)

0.72

$1,397.05$

$1,397.77$

\section{VOLUME PERCENTAGE BY DISPOSAL SITE \\ (Percentages $<.1 \%$ are not displayed)}

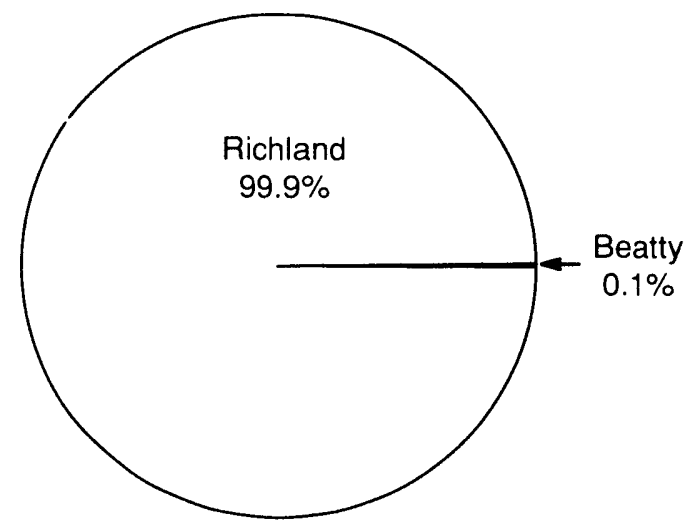

ACTIV!TY PERCENTAGE BY DISPOSAL SITE

(Percentages <. $1 \%$ are not displayed)

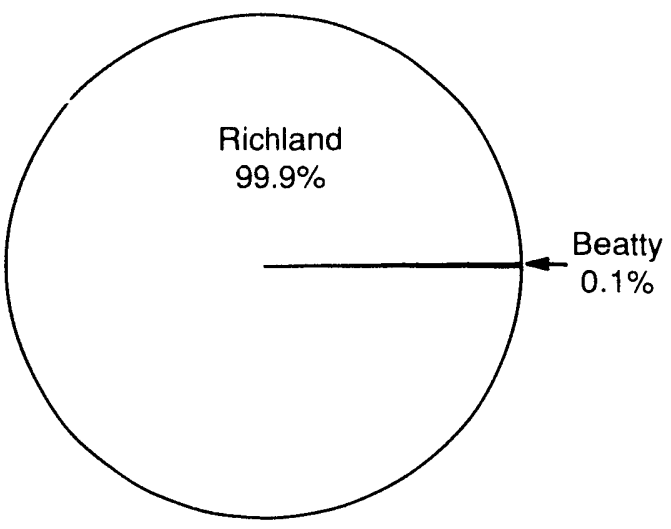

Volume Percentage by Waste Class for Direct Shipments and Total Non-Direct Volume Percentage (Percentages <. $1 \%$ are not displayed)
Direct Shipment

Waste Class

A

B

C

Total

Non-Direct Shipments

GrandTotal

Total Direct Shipment

Activity (curies)

Total Non-Direct Shipment

Activity (curies)

Grand Total (curies)
Volume

$\left(\mathrm{ft}^{3}\right)$

$22,029.72$

707.80

0.00

$22,737.52$

$3,403.57$

$26,141.09$

$1,383.26$

9.51

$1,397.77$

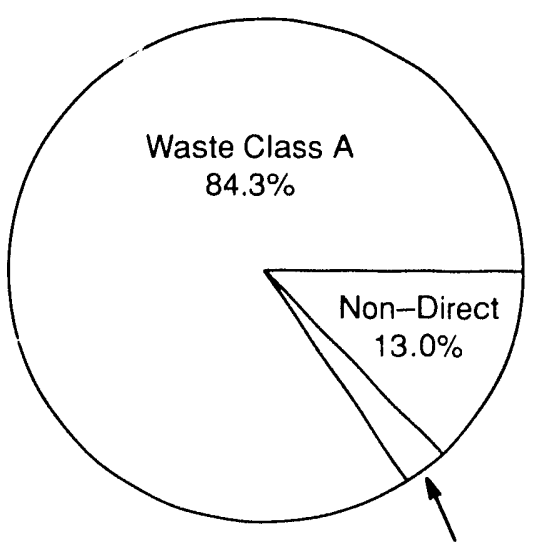

Waste Class B $2.7 \%$ 
I

I

I

I

I

I

I

I

I

I

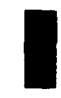

I

I

I

I

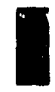

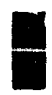

I 


\section{ROCKY MOUNTAIN COMPACT \\ Low-Level Radioactive Wastes Received at Commercial Disposal Sites}

\begin{tabular}{lrr}
\multicolumn{1}{c}{ States } & \multicolumn{1}{c}{$\begin{array}{c}\text { Volume } \\
\left(\mathrm{ft}^{3}\right)\end{array}$} & \multicolumn{1}{c}{$\begin{array}{c}\text { Activity } \\
\text { (curies) }\end{array}$} \\
Colorado & $2,309.16$ & $1,451.28$ \\
Nevada & 313.44 & 0.73 \\
New Mexico & $1,843.54$ & 0.88 \\
Wyoming & 15.00 & 1.88 \\
\cline { 2 - 2 } Total & $4,481.14$ & $1,454.78$
\end{tabular}

VOLUME PERCENTAGE BY STATE

(Percentages <. $1 \%$ are not displayed)

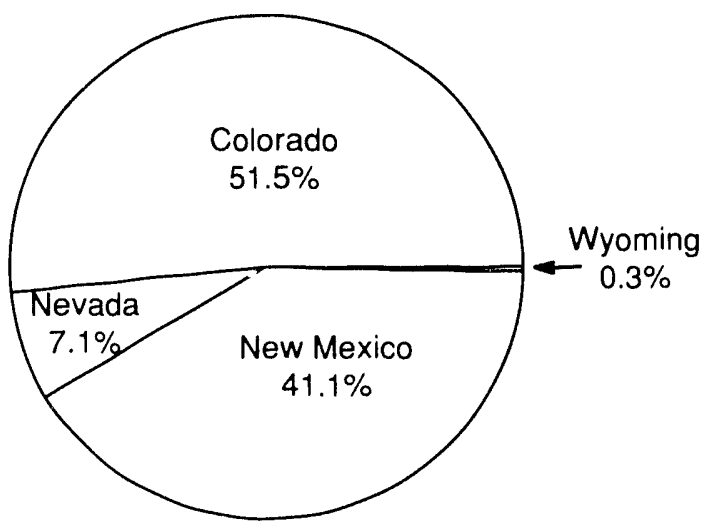

VOLUME PERCENTAGE BY CATEGORY

(Percentages <. $1 \%$ are not displayed)

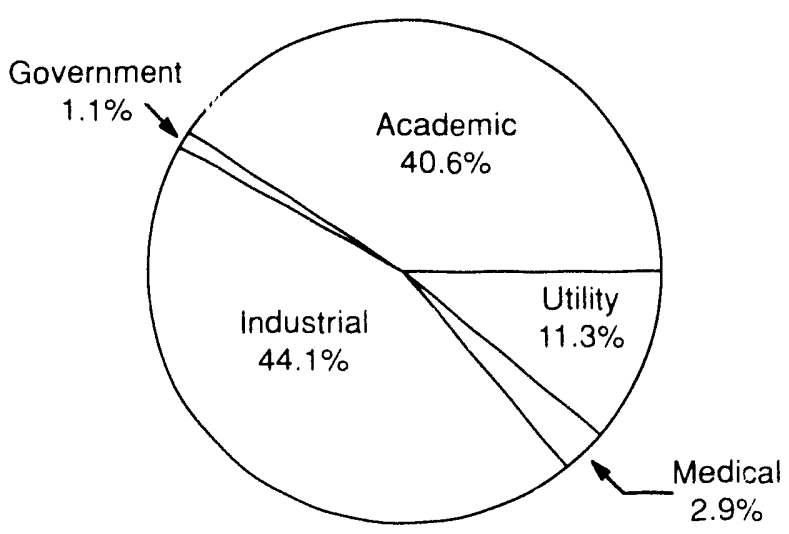




\section{COLORADO \\ Low-Level Radioactive Wastes Received at Commercial Disposal Sites}

\begin{tabular}{l} 
Generator \\
Category \\
\hline
\end{tabular}

Academic

Government

Industrial

Medical

Utility

Total

$\begin{array}{r}\begin{array}{c}\text { Volume } \\ \left(\mathrm{ft}^{3}\right)\end{array} \\ \hline 933.70 \\ 47.35 \\ 704.08 \\ 117.73 \\ 506.30 \\ \hline 2,309.16\end{array}$

Activity

(curies)

7.32

0.11

$1,443.25$

0.18

0.42

$1,451.28$

VOLUME PERCENTAGE BY SOURCE

ACTIVITY PERCENTAGE BY SOURCE

(Percentages <. $1 \%$ are not displayed)

(Percentages <. $1 \%$ are not displayed)
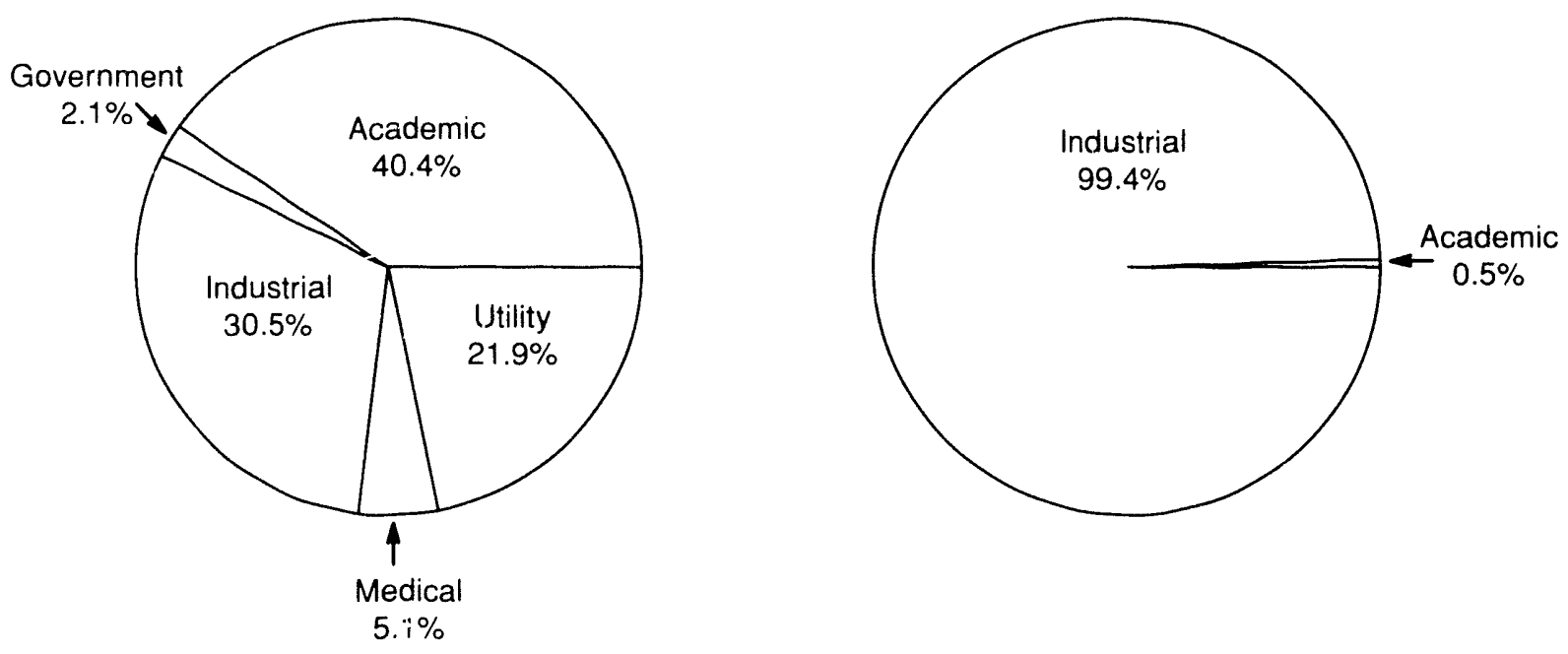


\section{COLORADO \\ Low-Level Radioactive Wastes Received at Commercial Disposal Sites}

Disposal
Site

Beatty

Richland

Total

\begin{tabular}{c}
$\begin{array}{c}\text { Volume } \\
\left(\mathrm{ft}^{3}\right)\end{array}$ \\
\hline $\begin{array}{r}1,963.18 \\
345.98\end{array}$ \\
\hline $2,309.16$
\end{tabular}

Activity

(curies)

$1,451.23$

0.05

$1,451.28$
VOLUME PERCENTAGE BY DISPOSAL SITE

(Percentages <.1\% are not displayed)

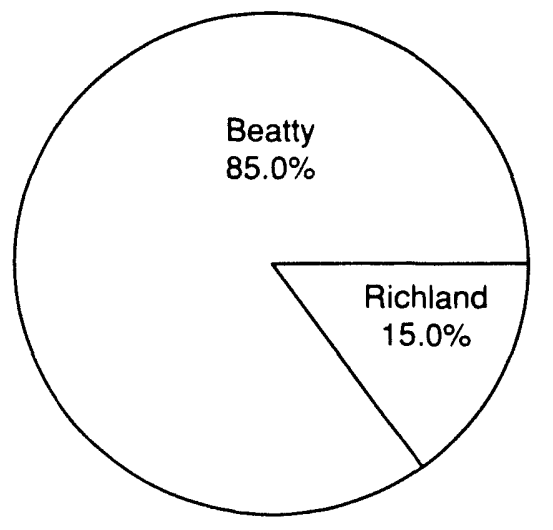

ACTIVITY PERCENTAGE BY DISPOSAL SITE

(Percentages $<.1 \%$ are not displayed)

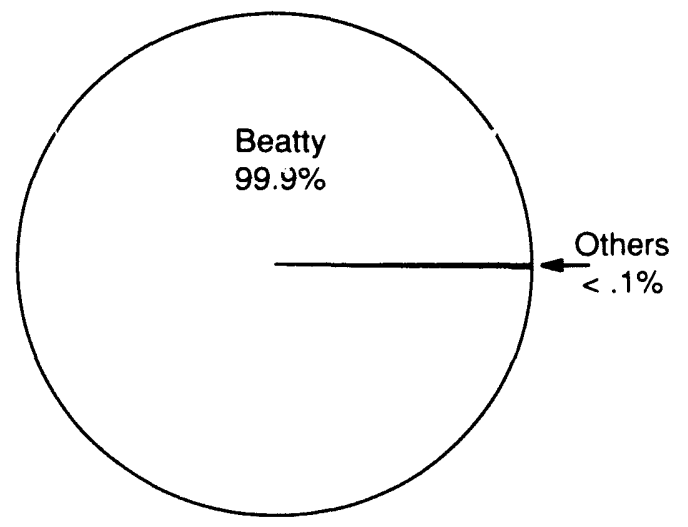

Volume Percentage by Waste Class for Direct Shipments and Total Non-Direct Volume Percentage (Percentages $<.1 \%$ are not displayed)

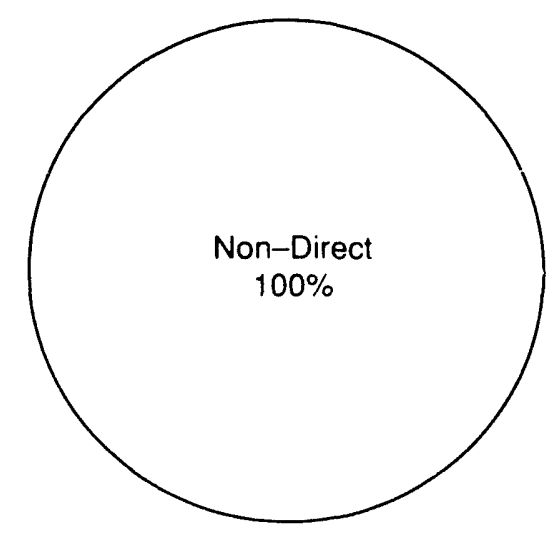




\section{NEVADA \\ Low-Level Radioactive Wastes Received at Commercial Disposal Sites}

\section{Generator \\ Category}

Academic

Government

Industrial

Medical

Total

$\begin{array}{r}\begin{array}{c}\text { Volume } \\ \left(\mathrm{ft}^{3}\right)\end{array} \\ \hline 286.70 \\ 0.50 \\ 15.16 \\ 11.08 \\ \hline\end{array}$

313.44
Activity (curies)

\begin{tabular}{l}
0.51 \\
0.13 \\
0.01 \\
0.08 \\
\hline 0.73
\end{tabular}

VOLUME PERCENTAGE BY SOURCE

(Percentages <. $1 \%$ are not displayed)

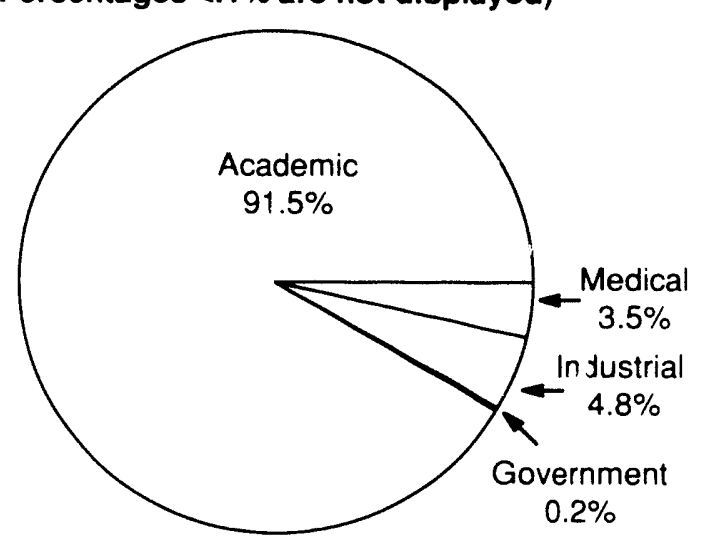

ACTIVITY PERCENTAGE BY SOURCE

(Percentages $<.1 \%$ are not displayed)

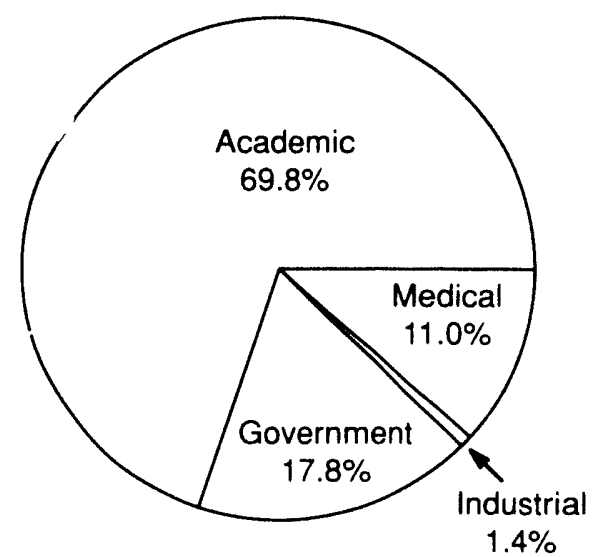




\title{
NEVADA \\ Low-Level Radioactive Wastes Received at Commercial Disposal Sites
}

$\begin{array}{lcr}\begin{array}{c}\text { Disposal } \\ \text { Site }\end{array} & \begin{array}{c}\text { Volume } \\ \left(\mathrm{ft}^{3}\right)\end{array} & \begin{array}{r}\text { Activity } \\ (\text { curies })\end{array} \\ \text { Beatty } & 313.44 & 0.73 \\ \text { Total } & 313.44 & 0.73\end{array}$

VOLUME PERCENTAGE BY DISPOSAL SITE

(Percentages <. $1 \%$ are not displayed)

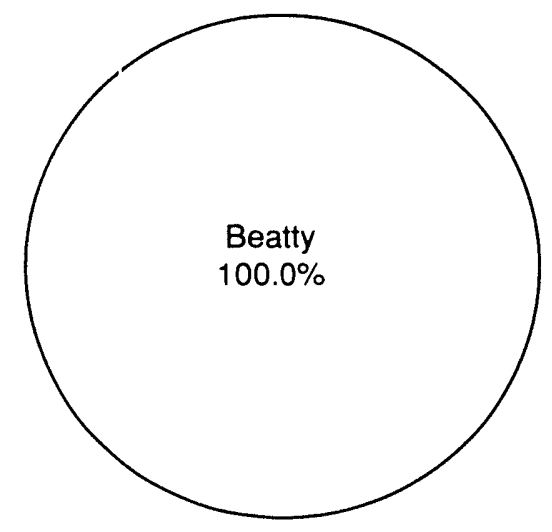

ACTIVITY PERCENTAGE BY DISPOSAL SITE

(Percentages <.1\% are not displayed)

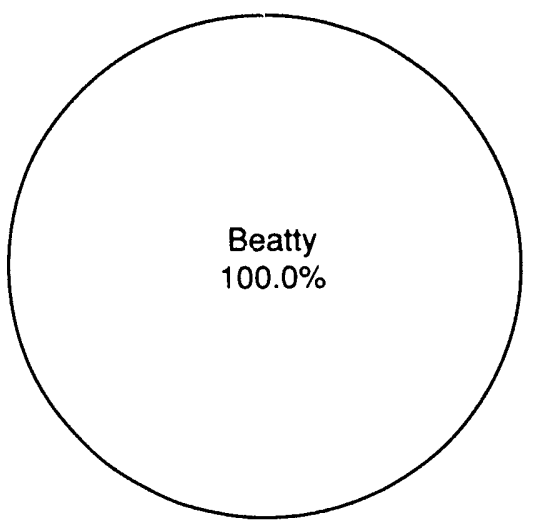

\begin{abstract}
Volume Percentage by Waste Class
for Direct Shipments and Total

Non-Direct Volume Percentage

(Percentages <. $1 \%$ are not displayed)
\end{abstract}

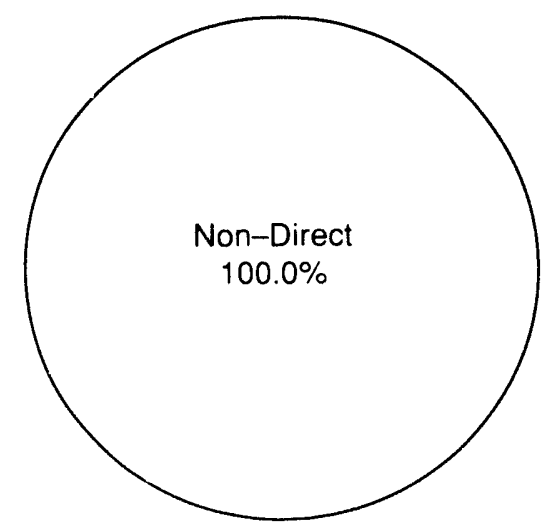




\section{NEW MEXICO \\ Low-Level Radioactive Wastes Received at Commercial Disposal Sites}

\begin{tabular}{l} 
Generator \\
Category \\
\hline
\end{tabular}

Academic

Industrial

Total

Volume
$\left(\mathrm{ft}^{3}\right)$

600.00

1.243 .54

1.843 .54
Activity

(curies)

0.69

0.19

0.88
VOLUME PERCENTAGE BY SOURCE (Percentages <. $1 \%$ are not displayed)

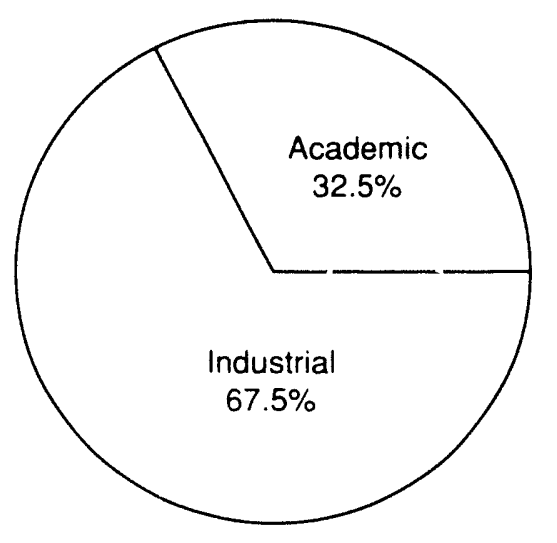

ACTIVITY PERCENTAGE BY SOURCE (Percentages <.1\% are not displayed)

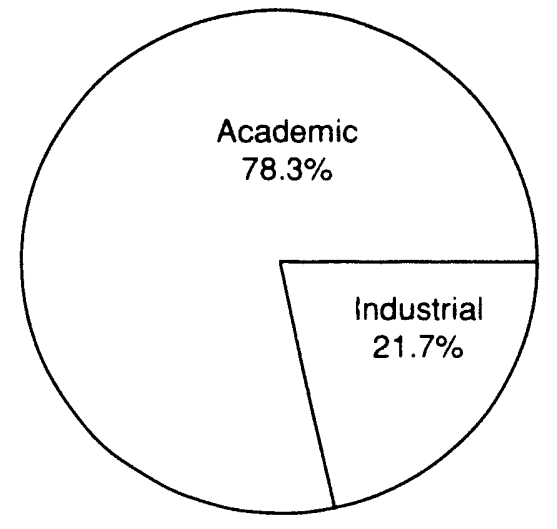




\section{NEW MEXICO \\ Low-Level Radioactive Wastes Received at Commercial Disposal Sites}

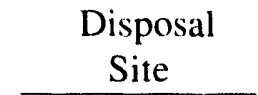

Beatty

Total

\author{
Volume \\ $\left(\mathrm{ft}^{3}\right)$
}

$1,843.54$

$1,843.54$
Activity

(curies)

0.88

0.88
VOLUME PERCENTÁGE BY DISPOSAL SITE (Percentages $<.1 \%$ are not displayed)

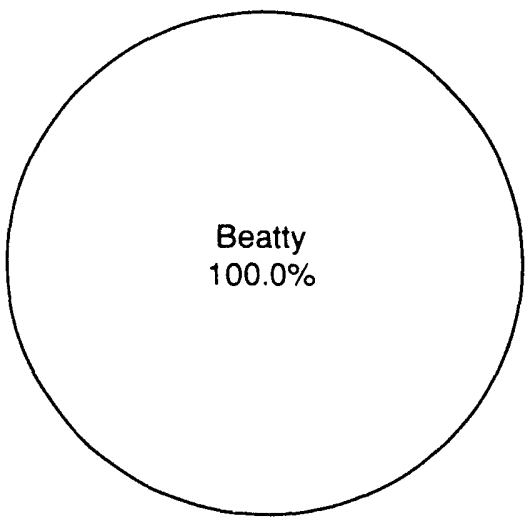

Direct Shipment Waste Class
A
B
C

Total

Non-Direct Shipments

Grand Total

Total Direct Shipment

Activity (curies)

Total Non-Direct Shipment

Activity (curies)

Grand Total (curies)
ACTIVITY PERCENTAGE BY DISPOSAL SITE (Percentages <. $1 \%$ are not displayed)

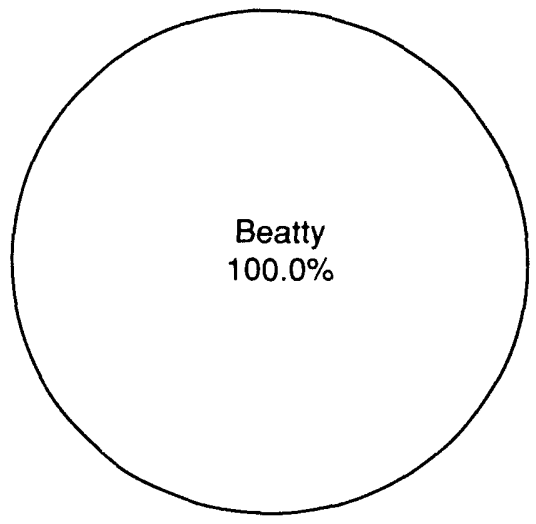

Volume Percentage by Waste Class for Direct Shipments and Total Non-Direct Volume Percentage (Percentages $<.1 \%$ are not displayed)

Volume $\left(\mathrm{ft}^{3}\right)$

$1,230.00$

0.00

0.00

$1,230.00$

613.54

$1,843.54$

0.72

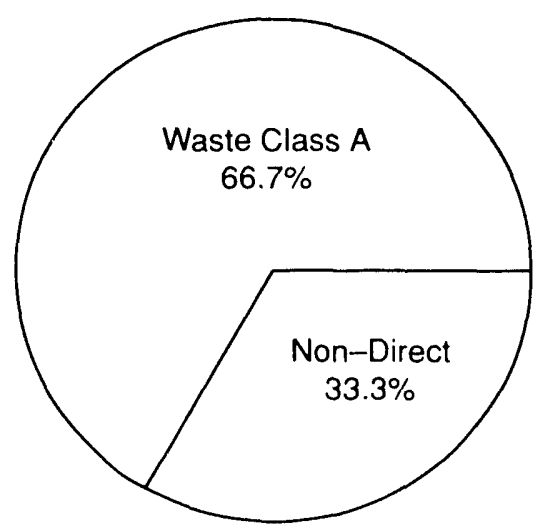

0.16

0.88 
WYOMING

\section{Low-Level Radioactive Wastes Received} at Commercial Disposal Sites

\section{Generator \\ Category}

Industrial

Total

\begin{tabular}{c}
$\begin{array}{c}\text { Volume } \\
\left(\mathrm{ft}^{3}\right)\end{array}$ \\
\hline
\end{tabular}

15.00

15.00
Activity

(curies)

1.88

1.88

VOLUME PERCENTAGE BY SOURCE

(Percentages $<.1 \%$ are not displayed)

(Percentages <. $1 \%$ are not displayed)
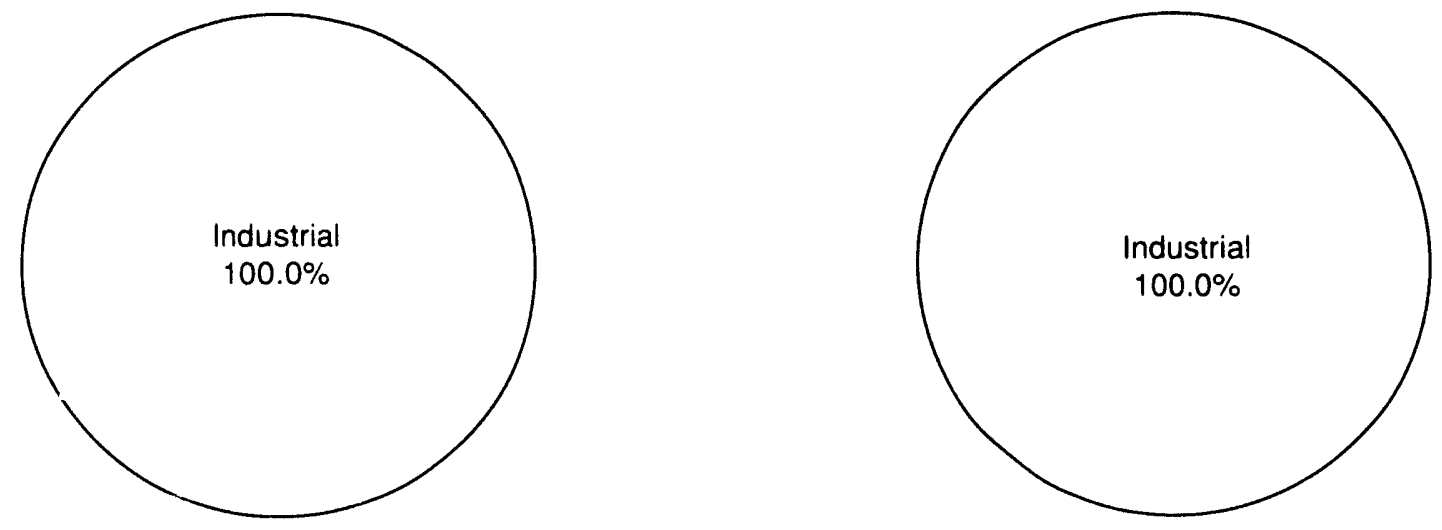


\section{WYOMING \\ Low-Level Radioactive Wastes Received at Commercial Disposal Sites}

$\begin{array}{lcr}\begin{array}{c}\text { Disposal } \\ \text { Site }\end{array} & \begin{array}{c}\text { Volume } \\ \left(\mathrm{ft}^{3}\right)\end{array} & \begin{array}{c}\text { Activity } \\ \text { (curies) }\end{array} \\ \text { Beatty } & 15.00 & 1.88 \\ \text { Total } & 15.00 & 1.88\end{array}$

VOLUME PERCENTAGE BY DISPOSAL SITE

(Percentages $<.1 \%$ are not displayed)

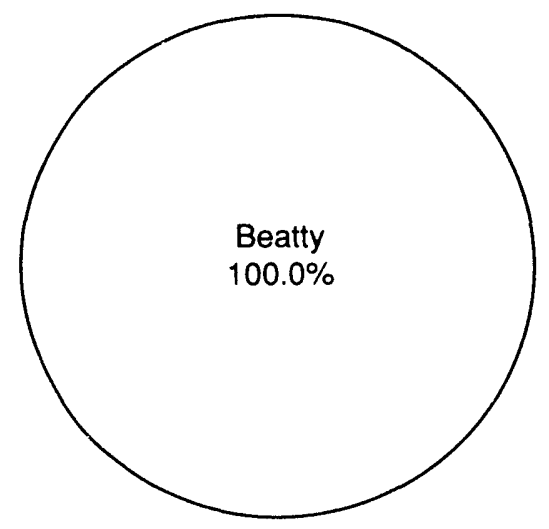

ACTIVITY PERCENTAGE BY DISPOSAL SITE

(Percentages <.1\% are not displayed)

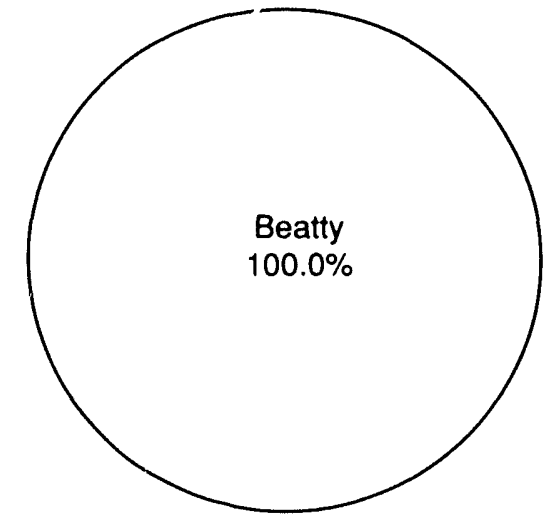

Volume Percentage by Waste Class

for Direct Shipments and Total

Non-Direct Volume Percentage

(Percentages $<.1 \%$ are not displayed)

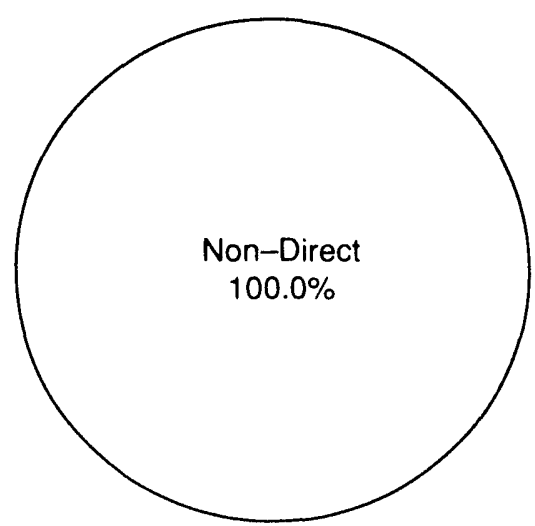


】

I

I

I

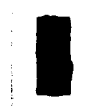

I

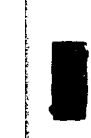

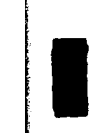

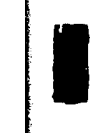

I

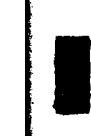

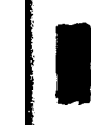

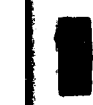

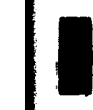

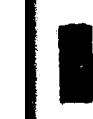




\section{SOUTHEAST COMPACT \\ Low-Level Radioactive Wastes Received at Commercial Disposal Sites}

\begin{tabular}{lrr}
\multicolumn{1}{c}{ States } & \multicolumn{1}{c}{$\begin{array}{c}\text { Volume } \\
\left(\mathrm{ft}^{3}\right)\end{array}$} & \multicolumn{1}{c}{$\begin{array}{c}\text { Activity } \\
\text { (curies) }\end{array}$} \\
Alabama & $14,237.55$ & $4,857.73$ \\
Florida & $24,587.01$ & $6,808.40$ \\
Georgia & $55,286.71$ & $29,053.84$ \\
Mississippi & $5,981.24$ & $1,368.53$ \\
North Carolina & $48,097.71$ & $3,050.70$ \\
South Carolina & $46,927.41$ & $2,208.45$ \\
Tennessee & $83,401.85$ & $1,209.64$ \\
Virginia & $57,765.63$ & $1,923.95$ \\
Total & $336,285.11$ & \\
& &
\end{tabular}

VOLUME PERCENTAGE BY STATE

(Percentages <.1\% are not displayed) Mississippi

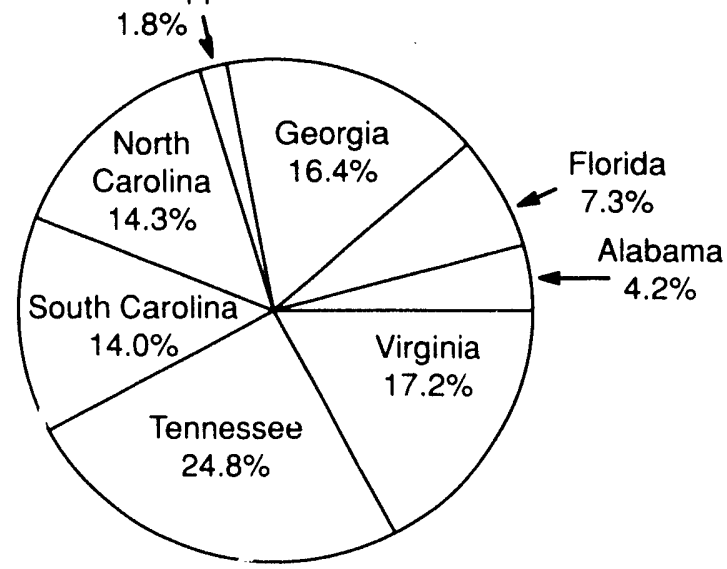

VOLUME PERCENTAGE BY CATEGORY

(Percentages $<.1 \%$ are not displayed)

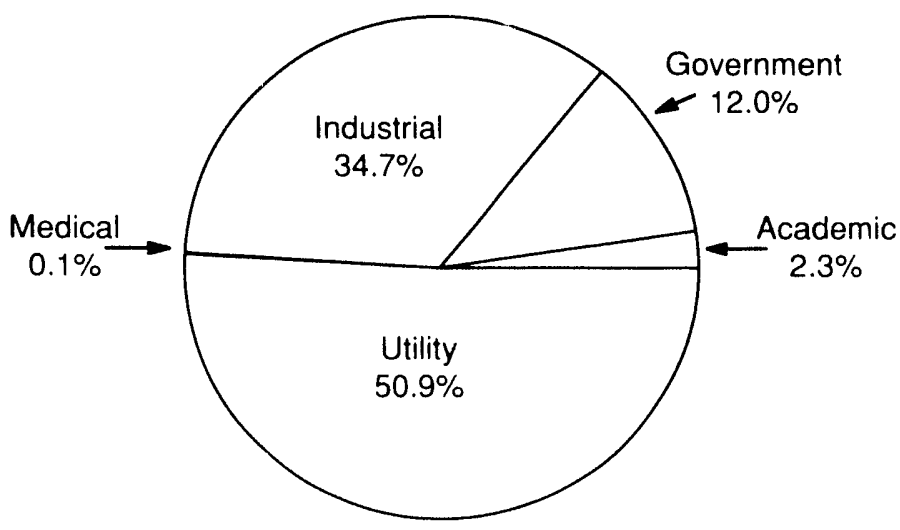




\section{ALABAMA \\ Low-Level Radioactive Wastes Received at Commercial Disposal Sites}

\section{Generator \\ Category}

Academic

Government

Industrial

Medical

Utility

Total
Volume

$\left(\mathrm{ft}^{3}\right)$

602.63

104.60

571.00

17.40

$12,941.92$

14.237 .55
Activity

(curies)

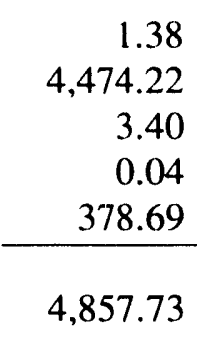

VOLUME PERCENTAGE BY SOURCE (Percentages <. $1 \%$ are not displayed)
ACTIVITY PERCENTAGE BY SOURCE

(Percentages $<.1 \%$ are not displayed)
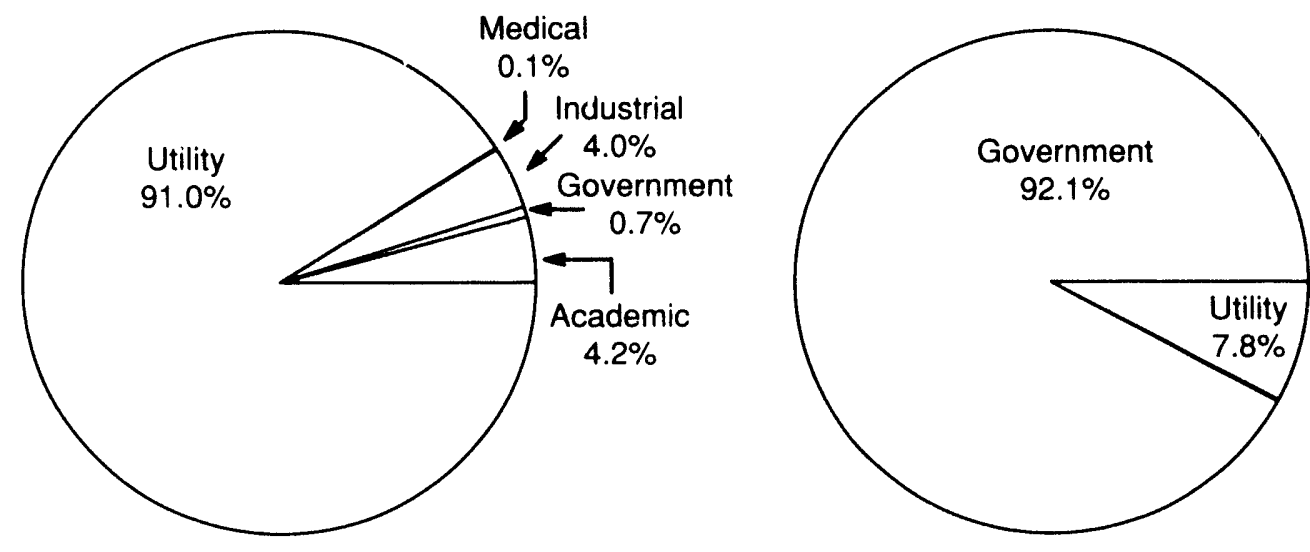


\section{ALABAMA \\ Low-Level Radioactive Wastes Received at Commercial Disposal Sites}

\begin{tabular}{|c|c|c|}
\hline $\begin{array}{c}\text { Disposal } \\
\text { Site }\end{array}$ & $\begin{array}{c}\text { Volume } \\
\left(\mathrm{ft}^{3}\right)\end{array}$ & $\begin{array}{l}\text { Activity } \\
\text { (curies) }\end{array}$ \\
\hline Baniwell & $13,723.54$ & $4,856.14$ \\
\hline Beatty & 4.01 & 0.03 \\
\hline Richland & 510.00 & 1.56 \\
\hline Total & $14,237.55$ & $4,857.73$ \\
\hline
\end{tabular}

VOLUME PERCENTAGE BY DISPOSAL SITE

(Percentages <. $1 \%$ are not displayed)

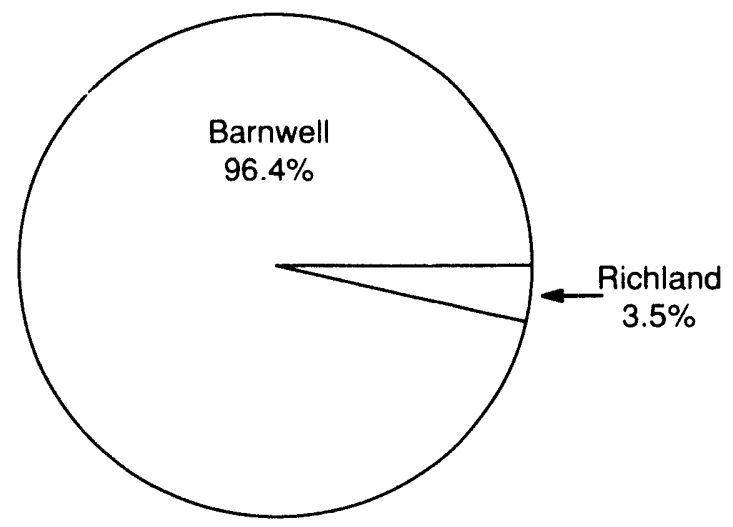

ACTIVITY PERCENTAGE BY DISPOSAL SITE

(Percentages <. $1 \%$ are not displayed)

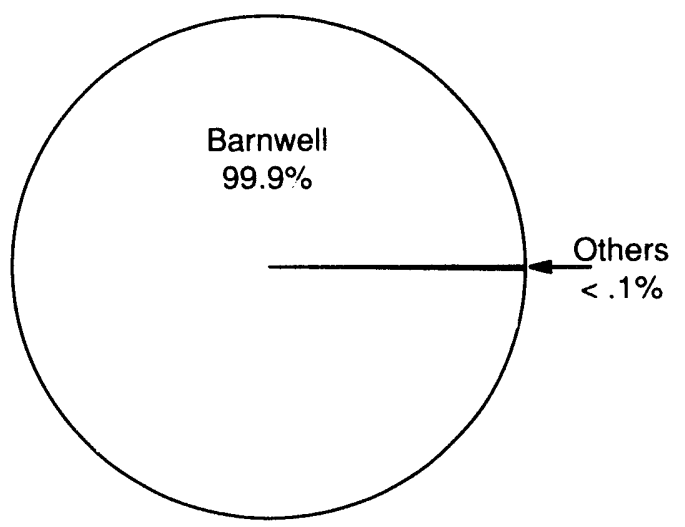

Volume Percentage by Waste Class for Direct Shipments and Total Non-Direct Volume Percentage (Percentages <. $1 \%$ are not displayed)
Direct Shipment

Waste Class

A

B

C

Total

Non-Direct Shipments

Grand Total

Total Direct Shipment

Activity (curies)

Total Non-Direct Shipment

Activity (curies)

Grand Total (curies)

$\begin{array}{r}\begin{array}{c}\text { Volume } \\ \left(\mathrm{ft}^{3}\right)\end{array} \\ \hline 5,326.00 \\ 558.40 \\ 76.60 \\ \hline 5,961.00 \\ 8,276.55 \\ \hline 14,237.55\end{array}$

365.59

$4,492.14$

$4,857.73$ 


\section{FLORIDA \\ Low-Level Radioactive Wastes Received at Commercial Disposal Sites}

\begin{tabular}{lrr}
$\begin{array}{c}\text { Generator } \\
\text { Category }\end{array}$ & \multicolumn{1}{c}{$\begin{array}{c}\text { Volume } \\
\left(\mathrm{ft}^{3}\right)\end{array}$} & \multicolumn{1}{c}{$\begin{array}{c}\text { Activity } \\
\text { (curies) }\end{array}$} \\
Academic & 866.45 & 2.88 \\
Government & 18.10 & 6.98 \\
Industrial & 857.59 & 0.84 \\
Medical & 41.47 & 0.78 \\
Utility & 22.803 .40 & $6,796.92$ \\
\hline Total & $24,587.01$ & $6,808.40$
\end{tabular}

VOLLIME PERCENTAGE BY SOURCE

ACTIVITY PERCENTAGE BY SOURCE

(Percentages <. $1 \%$ are not displayed)

(Percentages <. $1 \%$ are not displayed)
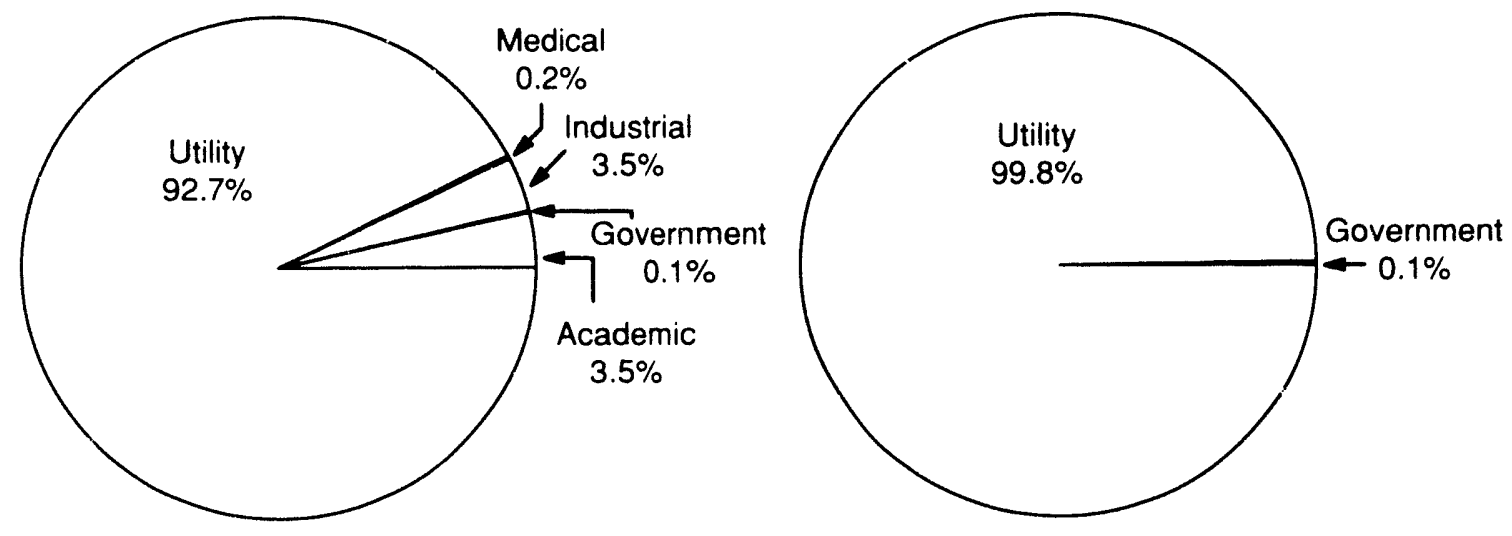


\section{FLORIDA \\ Low-Level Radioactive Wastes Received at Commercial Disposal Sites}

\begin{tabular}{lrr}
\multicolumn{1}{c}{$\begin{array}{c}\text { Disposal } \\
\text { Site }\end{array}$} & \multicolumn{1}{c}{$\begin{array}{c}\text { Volume } \\
\left(\mathrm{ft}^{3}{ }^{3}\right.\end{array}$} & \multicolumn{1}{c}{$\begin{array}{c}\text { Activity } \\
(\text { curies })\end{array}$} \\
Barnwell & & $6,807.73$ \\
Beatty & $24,518.04$ & 0.66 \\
Richland & 4.17 & 0.01 \\
Total & 64.80 & $6,808.40$
\end{tabular}

VOLUME PERCENTAGE BY DISPOSAL SITE

(Percentages <.1\% are not displayed)

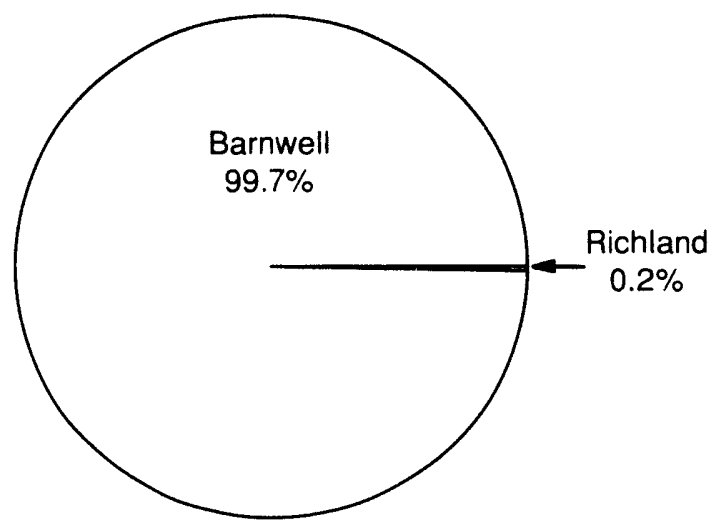

Direct Shipment

Waste Class

A
B
C

6.573 .10

866.30

261.10

$7,700.50$

Total

$16,886.51$

Non-Direct Shipments

$24,587.01$

Grand Total

6.770 .68

Activity (curies)

Total Non-Direct Shipment

Activity (curies)

Grand Total (curies)
ACTIVITY PERCENTAGE BY DISPOSAL SITE

(Percentages <. $1 \%$ are not displayed)

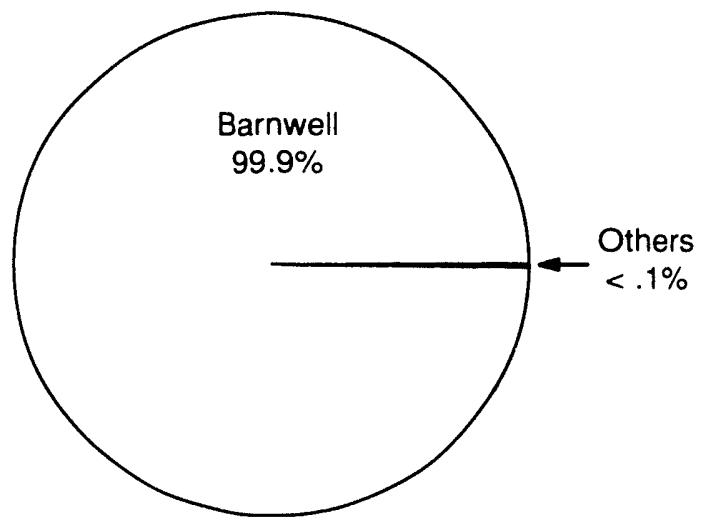

Volume Percentage by Waste Class for Direct Shipments and Total Non-Direct Volume Percentage (Percentages <. $1 \%$ are not displayed)

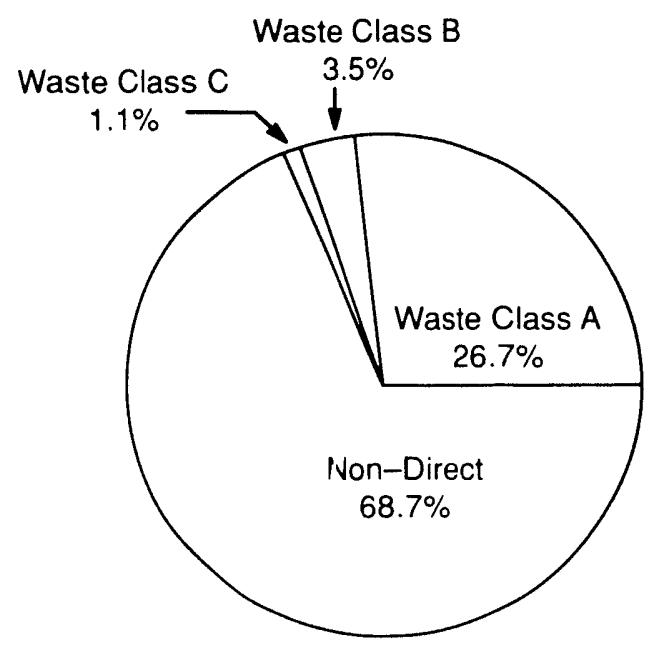




\section{GEORGIA \\ Low-Level Radioactive Wastes Received at Commercial Disposal Sites}

\begin{tabular}{l}
$\begin{array}{l}\text { Generator } \\
\text { Category }\end{array}$ \\
\hline Academic \\
Government \\
Industrial \\
Medical \\
Utility \\
Total
\end{tabular}

$\begin{array}{r}\begin{array}{c}\text { Volume } \\ \left(\mathrm{ft}^{3}\right)\end{array} \\ \hline 271.21 \\ 25.10 \\ 580.51 \\ 3.23 \\ 54.406 .66 \\ \hline\end{array}$

$55,286.71$
Activity
(curies)

$\begin{array}{r}17.24 \\ 7.46 \\ 5.72 \\ <.01 \\ 29,023.43 \\ \hline 29,053.84\end{array}$

VOLUME PERCENTAGE BY SOURCE (Percentages $<.1 \%$ are not displayed)

ACTIVITY PERCENTAGE BY SOURCE

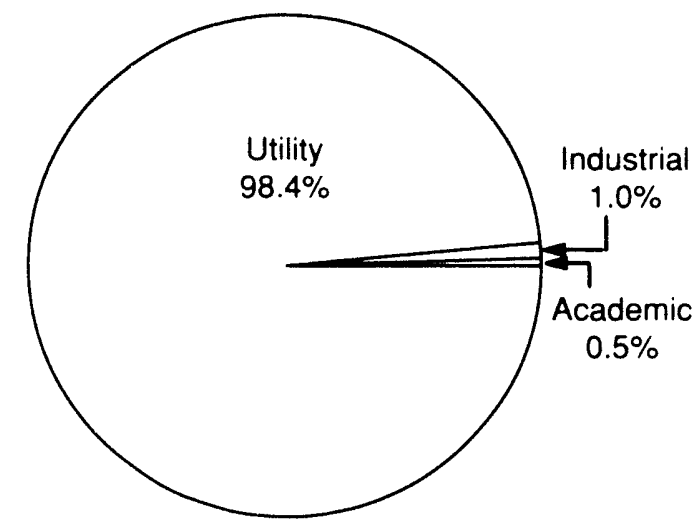
(Percentages <. $1 \%$ are not displayed)

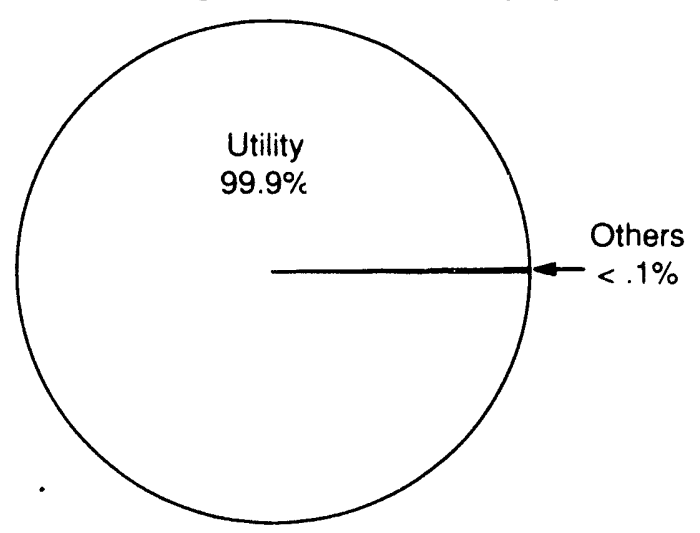




\section{GEORGIA \\ Low-Level Radioactive Wastes Received at Commercial Disposal Sites}

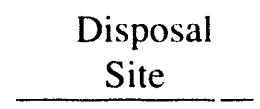

Barnwell

Total

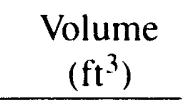

$55,286.71$

$55,286.71$
Activity

(curies)

$29,053.84$

$29,053.84$

\section{VOLUME PERCENTAGE BY DISPOSAL SITE} (percentages $<.1 \%$ are not displayed)

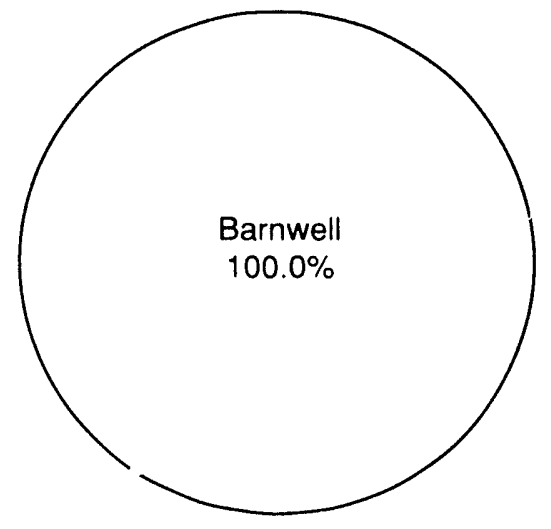

\section{ACTIVITY PERCENTAGE BY DISPOSAL SITE} (Percentages $<.1 \%$ are not displayed)

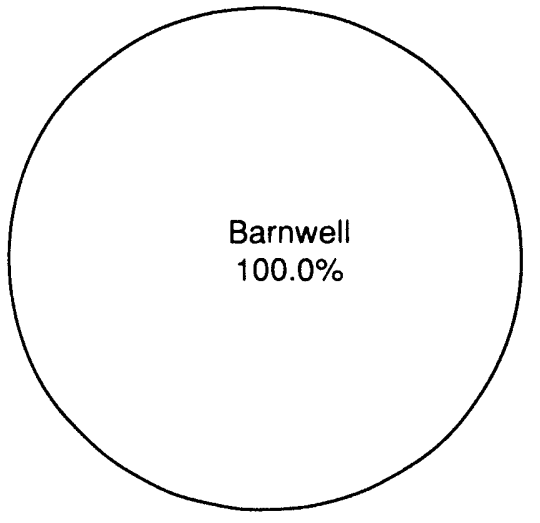

Volume Percentage by Waste Class for Direct Shipments and Total Non-Direct Volume Percentage (Percentages $<.1 \%$ are not displayed)
Direct Shipment

Waste Class

\section{A}

B

C

Total

Non-Direct Shipments

Grand Total

Total Direct Shipment

Activity (curies)

Total Non-Direct Shipment

Activity (curies)

Grand Total (curies)
Volume

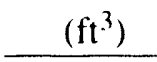

$42,745.38$

$2,057.80$

360.74

$45,163.92$

$10,122.79$

$55,286.71$

29.041 .73

12.11

$29,053.84$

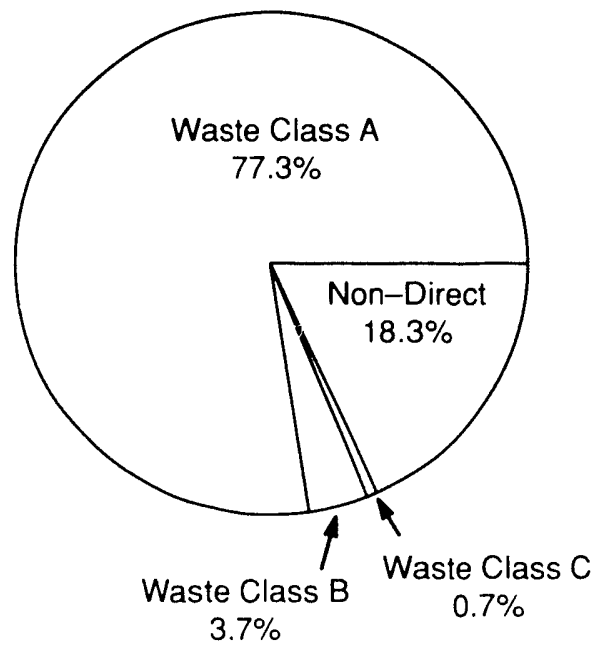




\section{MISSISSIPPI \\ Low-Level Radioactive Wastes Received at Commercial Disposal Sites}

\begin{tabular}{lrr}
$\begin{array}{l}\text { Generator } \\
\text { Category }\end{array}$ & \multicolumn{1}{c}{$\begin{array}{c}\text { Volume } \\
\left(\mathrm{ft}^{3}\right)\end{array}$} & $\begin{array}{r}\text { Activity } \\
\text { (curies) }\end{array}$ \\
& & $<.01$ \\
Academic & 18.36 & 17.75 \\
Government & 9.05 & 0.44 \\
Industrial & 230.18 & $<.01$ \\
Medical & 2.45 & $1,350.34$ \\
Utility & 5.721 .20 & $1,368.53$
\end{tabular}

VOLUME PERCENTAGE BY SOURCE

(Percentages $<.1 \%$ are not displayed)

ACTIVITY PERCENTAGE BY SOURCE

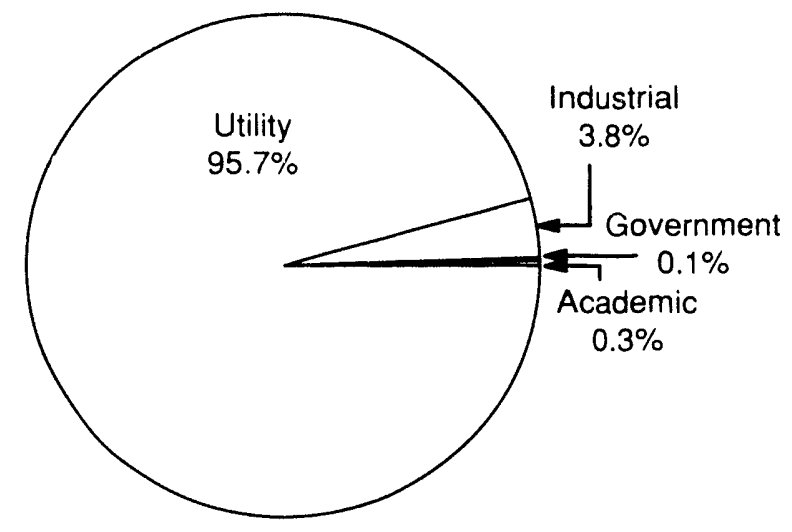

(Percentages <. $1 \%$ are not displayed)

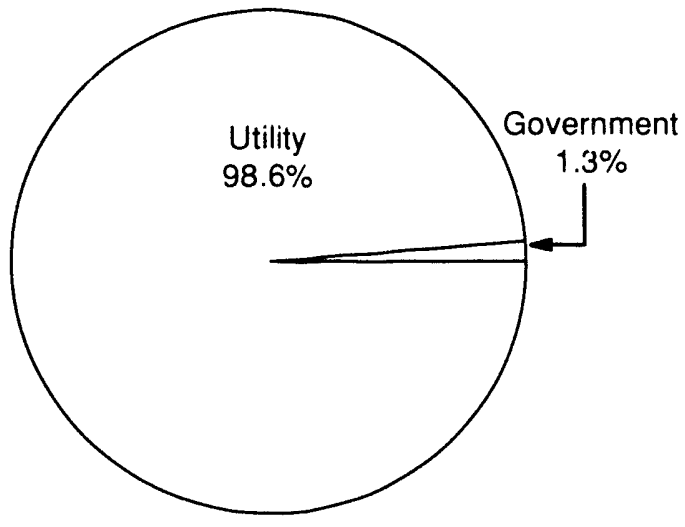




\section{MISSISSIPPI \\ Low-Level Radioactive Wastes Received at Commercial Disposal Sites}

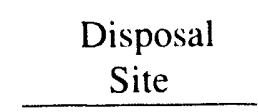

Barnwell

Total

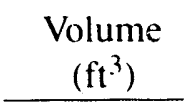

$5,981.24$

$5,981.24$
Activity

(curies)

$1,368.53$

$1,368.53$
VOLUME PERCENTAGE BY DISPOSAL SITE (Percentages <. $1 \%$ are not displayed)

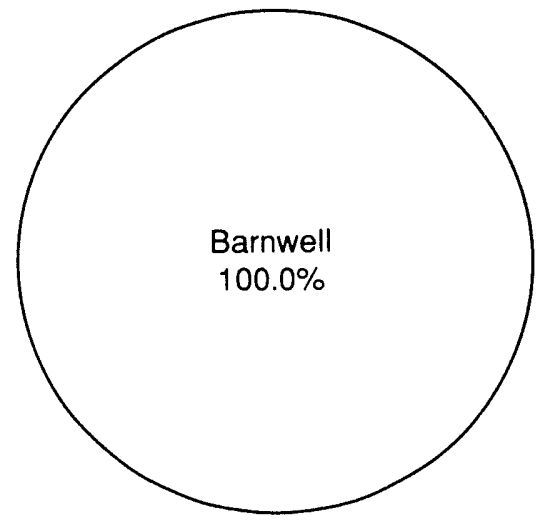

ACTIVITY PERCENTAGE BY DISPOSAL SITE (Percentages $<.1 \%$ are not displayed)

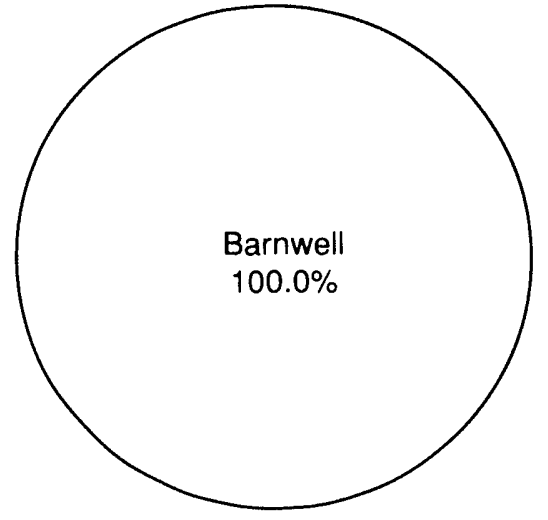

Volume Percentage by Waste Class for Direct Shipments and Total Non-Direct Volume Percentage (Percentages <. $1 \%$ are not displayed)
Direct Shipment Waste Class

A
B
C

Total

Non-Direct Shipments

Grand Total

Total Direct Shipment

Activity (curies)

Total Non-Direct Shipment

Activity (curies)

Grand Total (curies)
Volume $\left(\mathrm{ft}^{3}\right)$

$4,700.00$

0.00

0.00

$4,700.00$

$1,281.24$

$5,981.24$

$1,348.45$

20.09

$1,368.53$ 


\section{NORTH CAROLINA \\ Low-Level Radioactive Wastes Received at Commercial Disposal Sites}

\begin{tabular}{l}
$\begin{array}{l}\text { Generator } \\
\text { Category }\end{array}$ \\
\hline Academic \\
Government \\
Industrial \\
Medical \\
Utility \\
Total
\end{tabular}

VOLUME PERCENTAGE BY SOURCE

(Percentages <. $1 \%$ are not displayed)

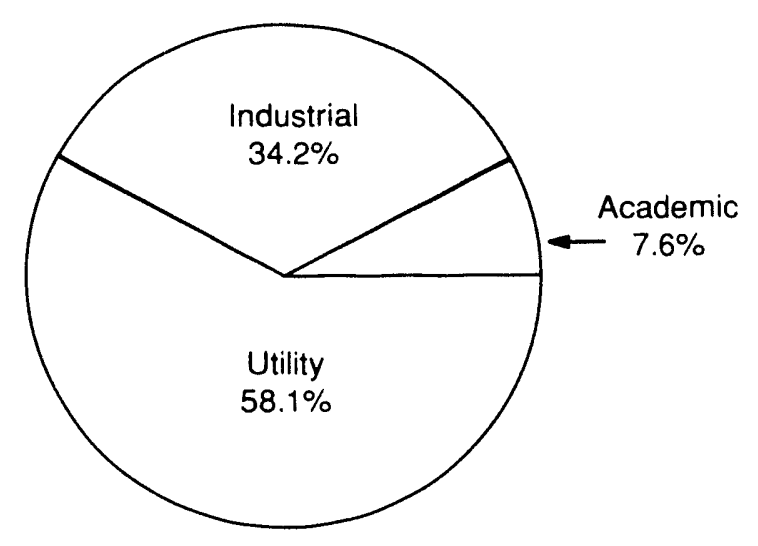

Volume

$\begin{array}{r}\multicolumn{1}{c}{\left(\mathrm{ft}^{3}\right)} \\ 3,668.31 \\ 30.60 \\ 16,440.75 \\ 33.70 \\ 27,924.35 \\ \hline\end{array}$

$48,097.71$
Activity

(curies)

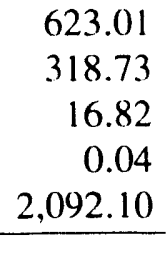

$3,050.70$

ACTIVITY PERCENTAGE BY SOURCE

(Perce :tages <. $1 \%$ are not displayed)

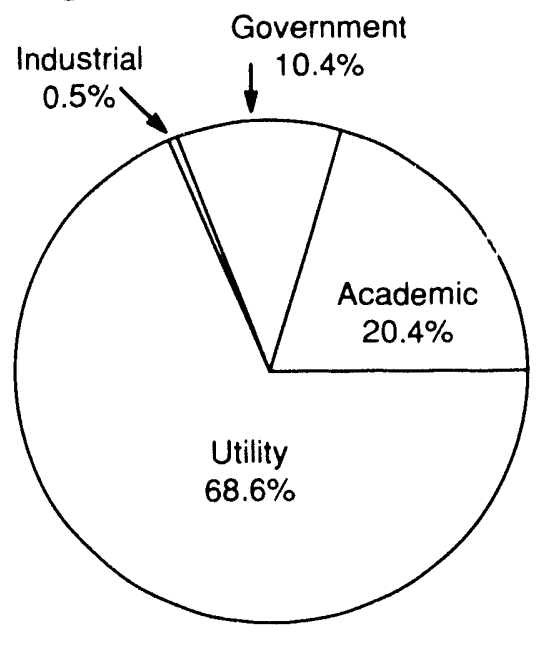




\section{NORTH CAROLINA \\ Low-Level Radioactive Wastes Received at Commercial Disposal Sites}

Disposal
Site

Barnwell

Beatty

Total

\author{
Volume \\ $\left(\mathrm{ft}^{3}\right)$
}

$48,078.67$

19.04

$48,097.71$
Activity

(curies)

$2,434.59$

616.11

$3,050.70$
VOLUME PERCENTAGE BY DISPOSAL SITE

(Percentages <. $1 \%$ are not displayed)

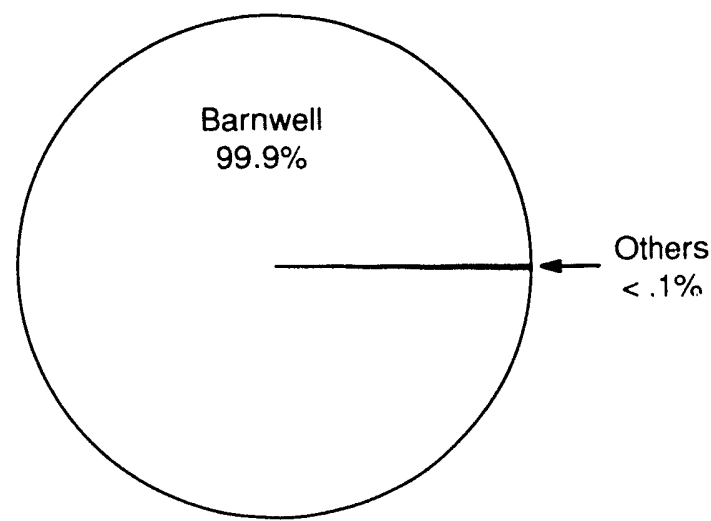

ACTIVITY PERCENTAGE BY DISPOSAL SITE

(Percentages <. $1 \%$ are not displayed)

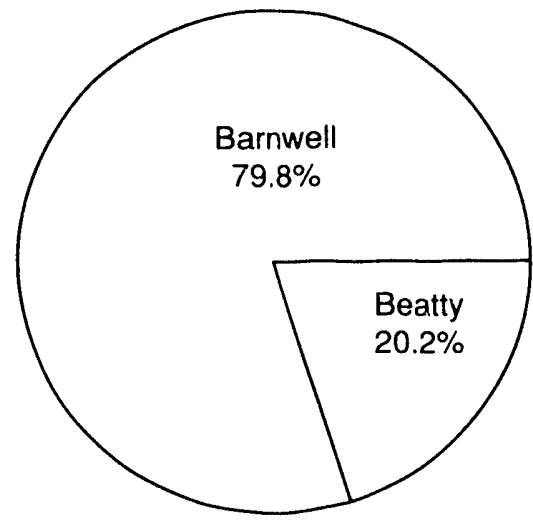

Volume Percentage by Waste Class for Direct Shipments and Total Non-Direct Volume Percentage (Percentages <. $1 \%$ are not displayed)
Direct Shipment

Waste Class

A

B

C

Total

Grand Total

Total Direct Shipment

Activity (curies)

Total Non-Dircct Shipment

Activity (curies)

Grand Total (curies)
Non-Direct Shipments
Volume

$\begin{array}{r}\multicolumn{1}{c}{\left(\mathrm{ft}^{3}\right)} \\ 30,295.84 \\ 675.20 \\ 132.96 \\ \hline 31,104.00 \\ 16,993.71 \\ \hline 48,097.71\end{array}$

$2,092.41$

958.29

$3,050.70$ 


\section{SOUTH CAROLINA \\ Low-Level Radioactive Wastes Received at Commercial Disposal Sites}

\begin{tabular}{l} 
Generator \\
Category \\
\hline
\end{tabular}

Academic Government Industrial Utility

Total

\begin{tabular}{c}
$\begin{array}{c}\text { Volume } \\
\left(\mathrm{ft}^{3}\right)\end{array}$ \\
\hline $1,082.91$ \\
7.536 .70 \\
$12,386.36$ \\
25.921 .44 \\
\hline
\end{tabular}

$46,927.41$

\author{
Activity \\ (curies)
}

0.72

137.70

21.18

$2,048.84$

$2,208.45$
VOLUME PERCENTAGE BY SOURCE (Percentages <. $1 \%$ are not displayed)

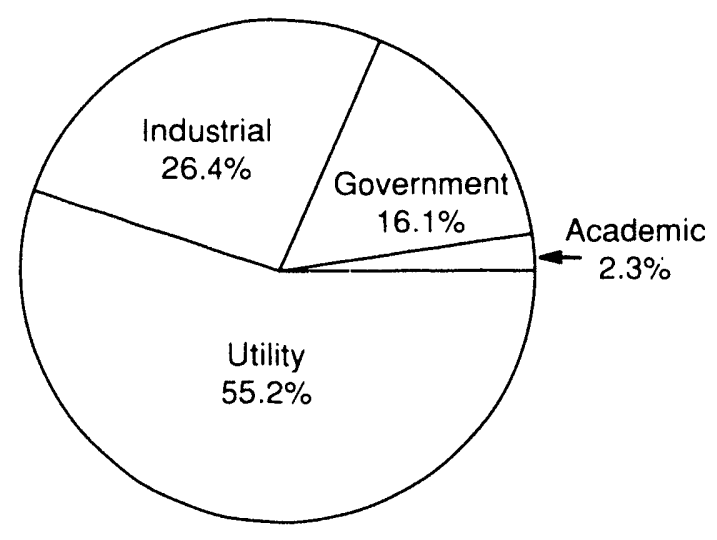

ACTIVITY PERCENTAGE BY SOURCE (Percentages <. $1 \%$ are not displayed)

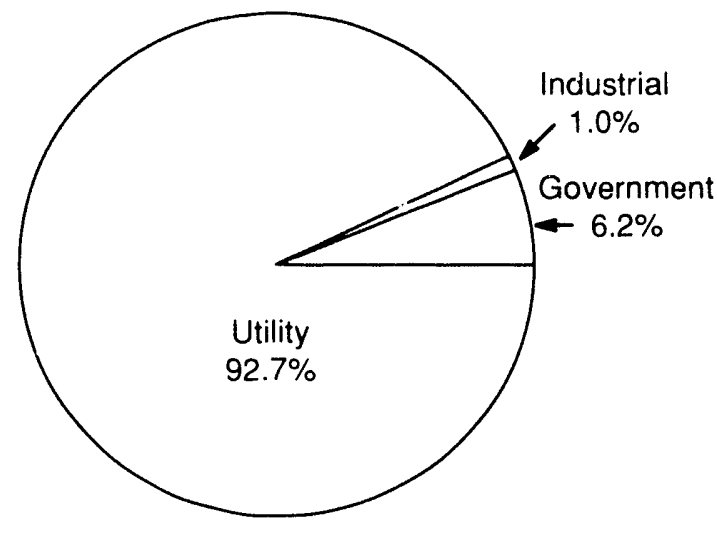




\section{SOUTH CAROLINA \\ Low-Level Radioactive Wastes Received at Commercial Disposal Sites}

\begin{tabular}{l} 
Disposal \\
Site \\
\hline
\end{tabular}

Barnwell

Richland

Total

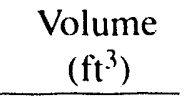

$46,919.91$

7.50

$46,927.41$
Activity

(curies)

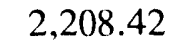

0.03

$2,208.45$
VOLUME PERCENTAGE BY DISPOSAL SITE (Percentages <. $1 \%$ are not displayed)

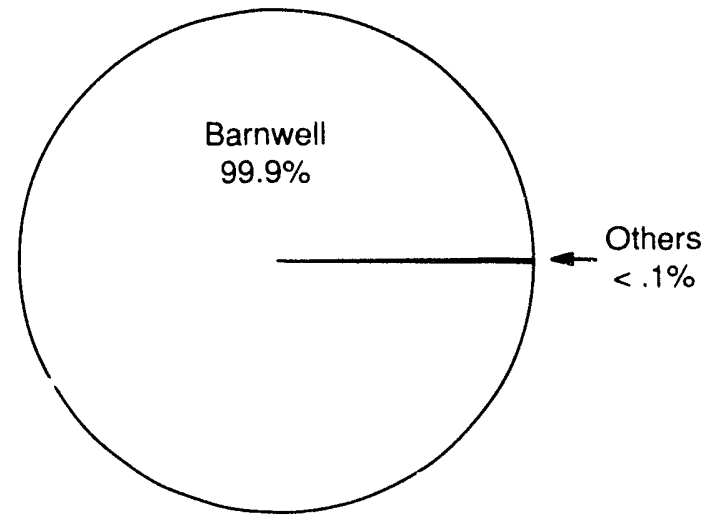

\section{ACTIVITY PERCENTAGE BY DISPOSAL SITE}

(Percentages <. $1 \%$ are not displayed)

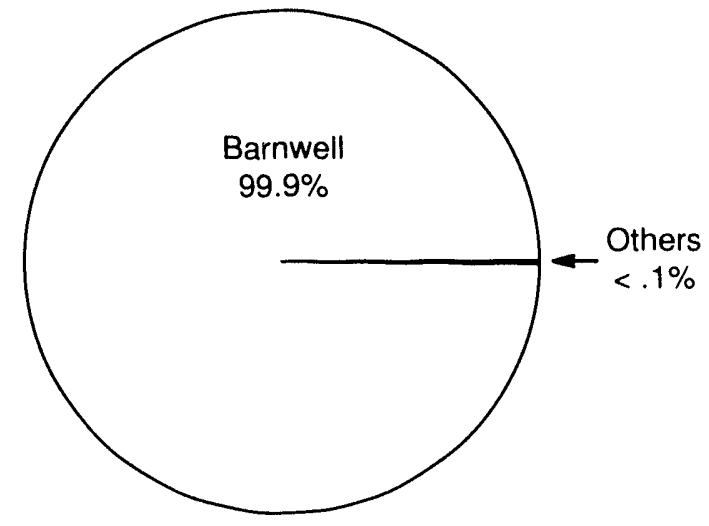

Volume Percentage by Waste Class

for Direct Shipments and Total

Non-Direct Volume Percentage

(Percentages $<.1 \%$ are not displayed)
Direct Shipment

Waste Class

A

B

C

Total

Non-Direct Shipments

Grand Total

Total Direct Shipment

Activity (curies)

Total Non-Direct Shipment

Activity (curies)

Grand Total (curies)
Volume $\left(\mathrm{ft}^{3}\right)$

$25,585.76$

828.00

741.70

$27,155.46$

19.771.95 Waste Class B

$46,927.41$

$2,183.71$

$1.8 \%$

Waste Class C

$1.6 \%$

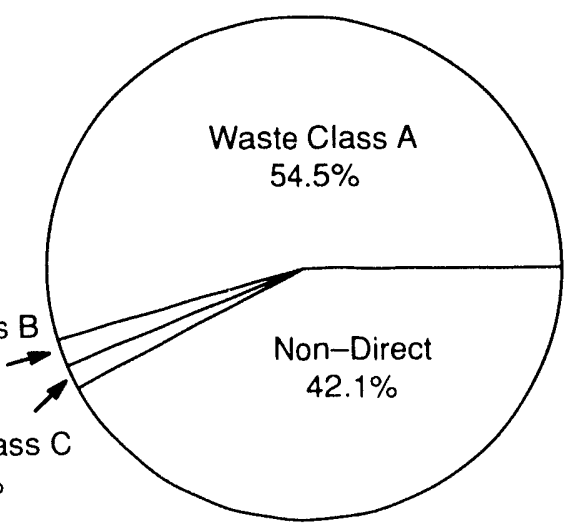

24.75

$2,208.45$ 


\section{TENNESSEE \\ Low-Level Radioactive Wastes Received at Commercial Disposal Sites}

\begin{tabular}{lrr}
$\begin{array}{c}\text { Generator } \\
\text { Category }\end{array}$ & \multicolumn{1}{c}{$\begin{array}{c}\text { Volume } \\
\left(\mathrm{ft}^{3}\right)\end{array}$} & \multicolumn{1}{c}{$\begin{array}{c}\text { Activity } \\
(\text { curies })\end{array}$} \\
\cline { 2 - 2 } Academic & 654.52 & 1.36 \\
Industrial & $73,240.61$ & 546.44 \\
Medical & 202.72 & 0.40 \\
Utility & $9,304.00$ & 661.45 \\
Total & $83,401.85$ & $1,209.64$
\end{tabular}

VOLUME PERCENTAGE BY SOURCE

(Percentages <. $1 \%$ are not displayed)

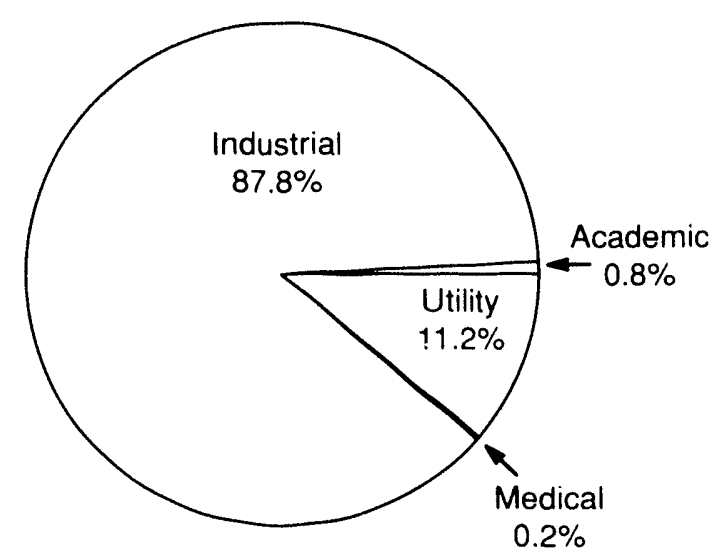

ACTIVITY PERCENTAGE BY SÚURCE

(Percentages $<.1 \%$ are not displayed)

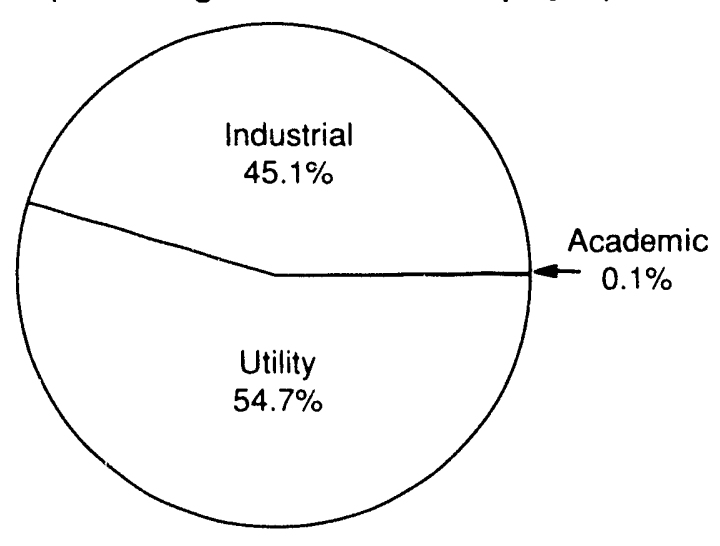




\section{TENNESSEE \\ Low-Level Radioactive Wastes Received at Commercial Disposal Sites}

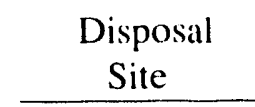

Barnwell

Beatty

Richland

Total

VOLUME PERCENTAGE BY DISPOSAL SITE

(Percentages <.1\% are not displayed)

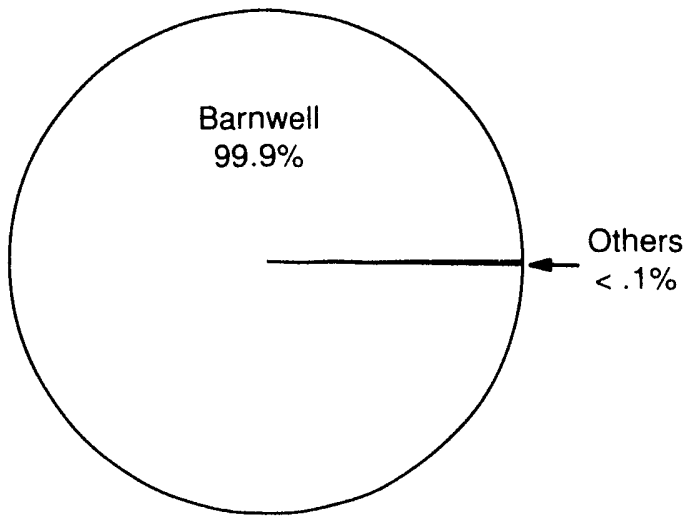

Direct Shipment

Waste Class

A

B

C

Total

Non-Direct Shipments

Grand Total

Total Direct Shipment

Activity (curies)

Total Non-Direct Shipment

Activity (curies)

Grand Total (curies)
Volume

$$
\left(\mathrm{ft}^{3}\right)
$$

$46,767.5()$

120.30

120.30

$47,008.10$

$36,393.75$

$83,401.85$

$1,153.85$

55.79

$1,209.64$

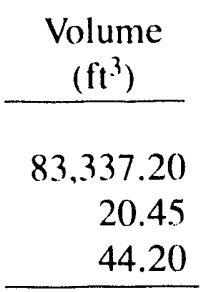

$83,401.85$
Activity (curies)

$1,209.34$

0.18

0.12

$1,209.64$
ACTIVITY PERCENTAGE BY DISPR,SAL SITE

(Percentages <. $1 \%$ are not displayed)

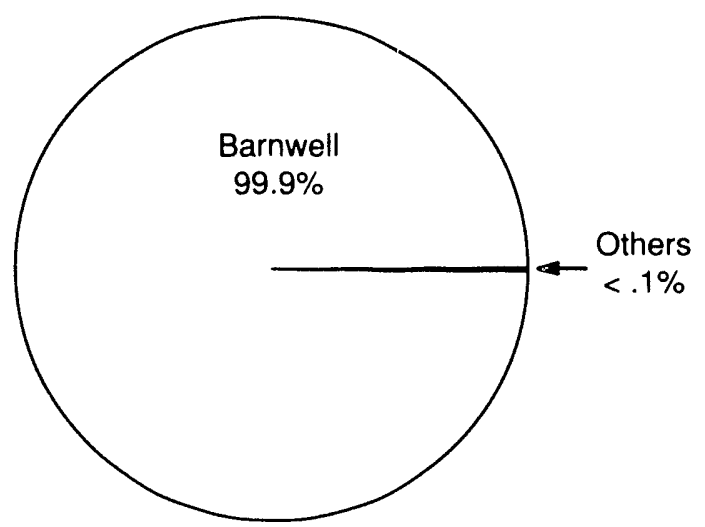

Volume Percentage by Waste Class for Direct Shipments and Total Non-Direct Volume Percentage (Percentages <. $1 \%$ are not displayed) 


\section{VIRGINIA \\ Low-l.evel Radioacitive Wastes Received at Commercial Disposal Sites}

\begin{tabular}{lrr}
$\begin{array}{l}\text { Generator } \\
\text { Category }\end{array}$ & $\begin{array}{c}\text { Volume } \\
\left(\mathrm{ft}^{3}\right)\end{array}$ & \multicolumn{1}{c}{$\begin{array}{c}\text { Activity } \\
\text { (curies) }\end{array}$} \\
Academic & 710.69 & 0.75 \\
Government & $32,388.90$ & 58.81 \\
Industrial & $12,380.65$ & 6.88 \\
Medical & 1.89 & 0.02 \\
Utility & $12,283.50$ & $1,857.50$ \\
Total & $57,765.63$ & $1,923.95$
\end{tabular}

VOLUME PERCENTAGE BY SOURCE

(Percentages <. $1 \%$ are not displayed)

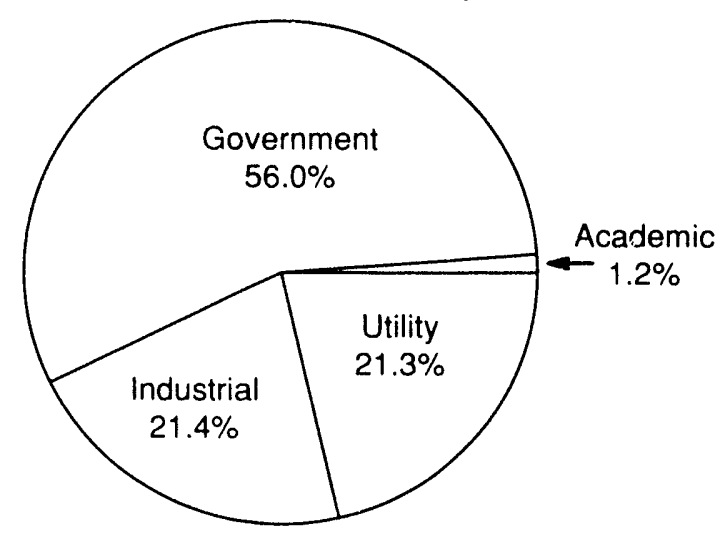

ACTIVITY PERCENTAGE BY SOURCE

(Percentages <. $1 \%$ are not displayed)

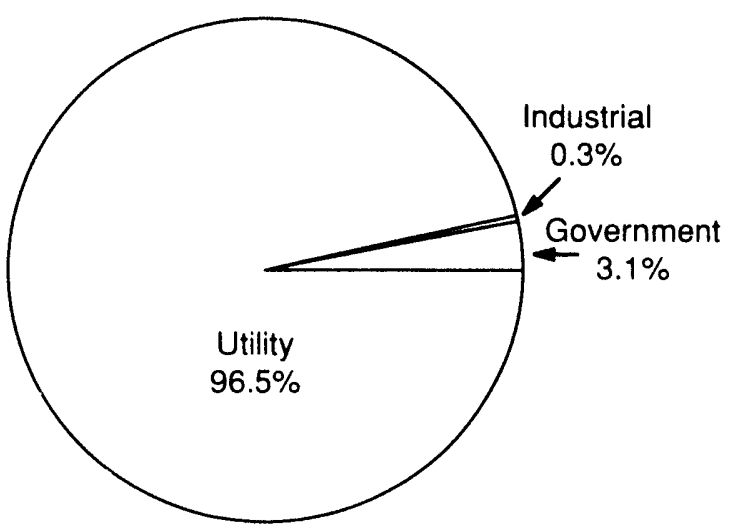




\section{VIRGINIA \\ Low-Level Radioactive Wastes Received at Commercial Disposal Sites}

\begin{tabular}{c} 
Disposal \\
Site \\
\hline
\end{tabular}

Barnwell

Beatty

Total

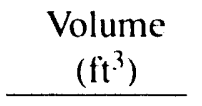

$57,762.93$

2.70

$57,765.63$
Activity

(curies)

$1,923.94$

0.02

$1,923.95$
VOLUME PERCENTAGE BY DISPOSAL SITE (Percentages <. $1 \%$ are not displayed)

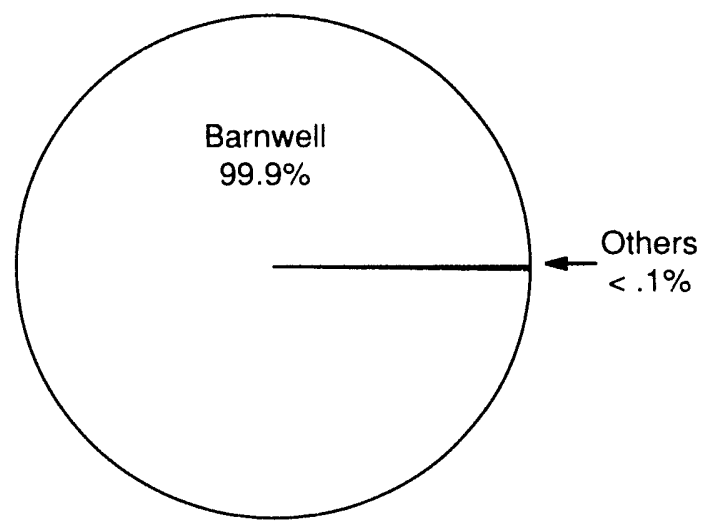

Direct Shipment Waste Class

A

B

C

Total

Non-Direct Shipments

Grand Total

Total Direct Shipment

Activity (curies)

Total Non-Direct Shipment

Activity (curies)

Grand Total (curies)
Volume

$$
\begin{array}{r}
\left(\mathrm{ft}^{3}\right) \\
\hline 41,592.00 \\
851.70 \\
360.90 \\
\hline 42,804.60 \\
14,961.03 \\
\hline 57,765.63
\end{array}
$$

$1,903.83$

20.12

$1,923.95$
ACTIVITY PERCENTAGE BY DISPOSAL SITE (Percentages <. $1 \%$ are not displayed)

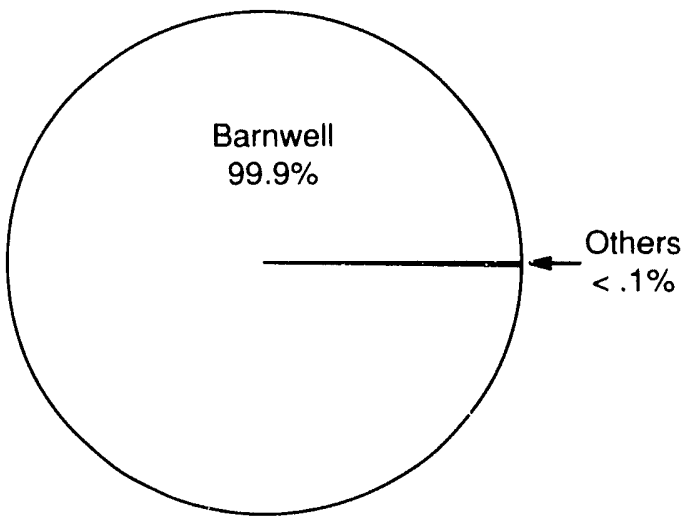

Volume Percentage by Waste Class for Direct Shipments and Total Non-Direct Volume Percentage (Percentages $<.1 \%$ are not displayed)

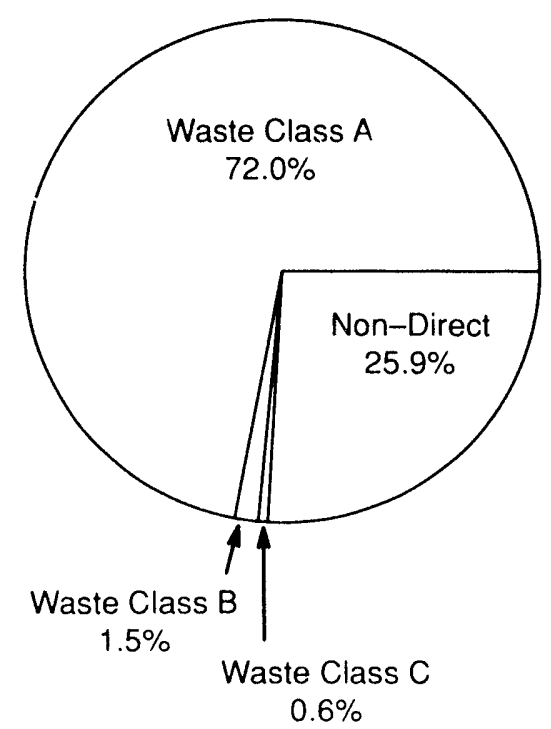


】

I

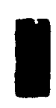

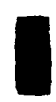

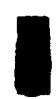

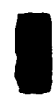

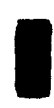

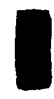

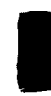

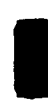

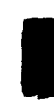

,

,

,

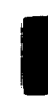

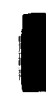

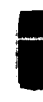

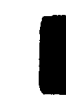




\section{SOUTHWESTERN COMPACT Low-Level Radioactive Wastes Received at Commercial Disposal Sites}

\begin{tabular}{lrr}
\multicolumn{1}{c}{ States } & \multicolumn{1}{c}{$\begin{array}{c}\text { Volume } \\
\left(\mathrm{ft}^{3}\right)\end{array}$} & \multicolumn{1}{c}{$\begin{array}{c}\text { Activity } \\
\text { (curies) }\end{array}$} \\
Arizona & $27,751.46$ & 246.85 \\
California & $55,956.54$ & $5,537.69$ \\
North Dakota & 32.90 & 0.02 \\
South Dakota & $1,169.64$ & 0.05 \\
Total & $84,910.54$ & $5,784.61$
\end{tabular}

VOLUME PERCENTAGE BY STATE

(Percentages $<.1 \%$ are not displayed)

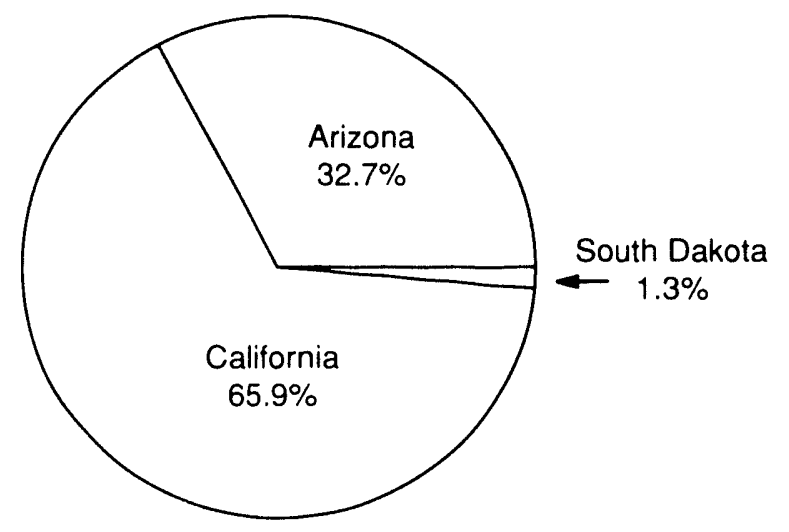

VOLUME PERCENTAGE BY CATEGORY

(Percentages $<.1 \%$ are not displayed)

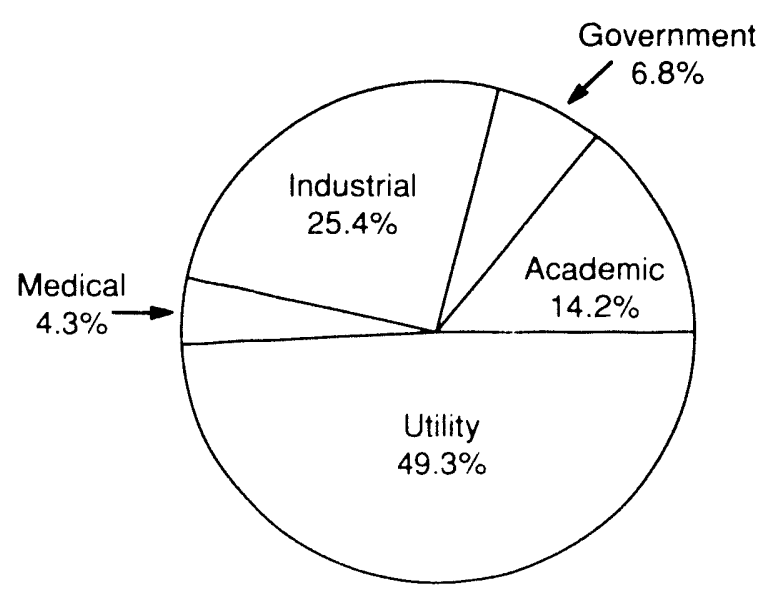




\section{ARIZONA \\ Low-Level Radioactive Wastes Received at Commercial Disposal Sites}

$\begin{array}{lrr}\begin{array}{l}\text { Generator } \\ \text { Category }\end{array} & \begin{array}{c}\text { Volume } \\ \left(\mathrm{ft}^{3}\right)\end{array} & \begin{array}{r}\text { Activity } \\ \text { (curies) }\end{array} \\ \text { Academic } & 665.80 & 4.42 \\ \text { Government } & 1.00 & <.01 \\ \text { Industrial } & 1.54 & <.01 \\ \text { Medical } & 5.52 & 0.51 \\ \text { Utility } & 27,077.60 & 241.92 \\ \text { Total } & 27,751.46 & 246.85\end{array}$

VOLUME PERCENTAGE BY SOURCE

(Percentages $<.1 \%$ are not displayed)

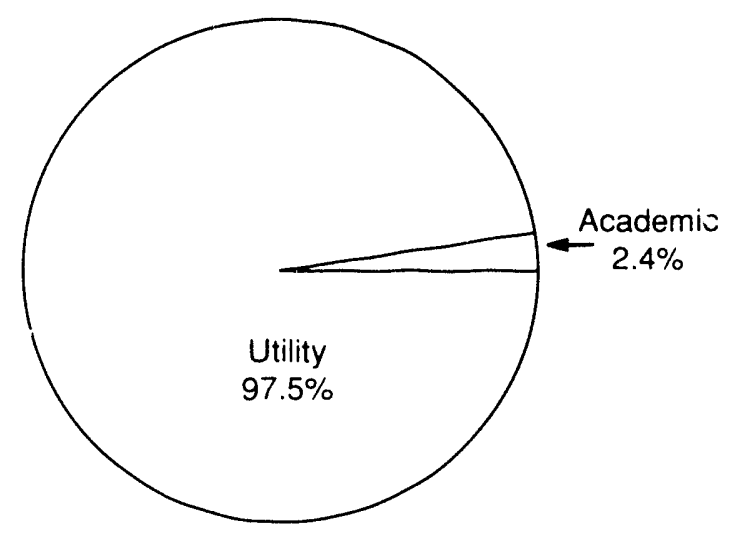

ACTIVITY PERCENTAGE BY SOURCE

(Percentages $<.1 \%$ are not displayed)

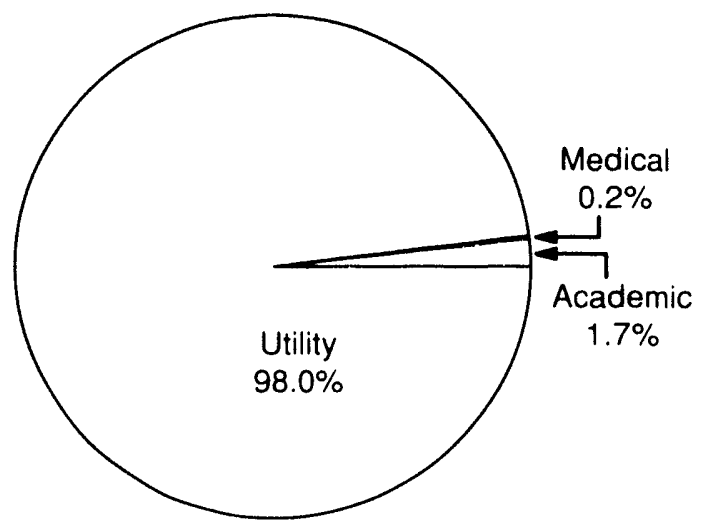




\section{ARIZONA \\ Low-Level Radioactive Wastes Received at Commercial Disposal Sites}

\begin{tabular}{c} 
Disposal \\
Site \\
\hline
\end{tabular}

Barnwell

Beatty

Richland

Total

\author{
Volume \\ $\left(\mathrm{ft}^{3}\right)$
}

0.50

$1,399.06$

$26,351.90$

$27,751.46$
Activity

(curies)

$$
<.01
$$

242.87

246.85

\section{VOLUME PERCENTAGE BY DISPOSAL SITE (Percentages <.1\% are not displayed)}

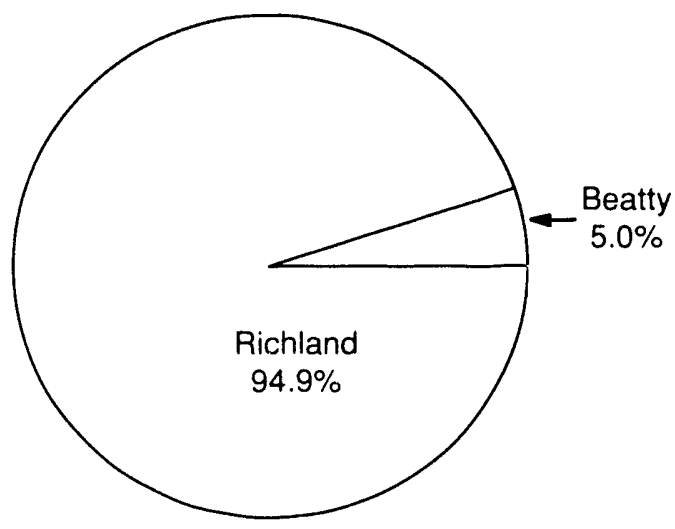

Direct Shipment

Waste Class
A
B
C

Total

Non-Direct Shipments

Grand Total

Total Direct Shipment

Activity (curies)

Total Non-Direct Shipment

Activity (curies)

Grand Total (curies)
Volume

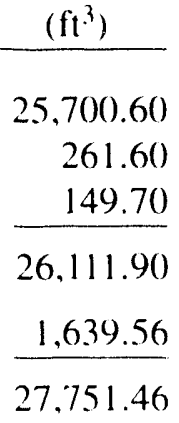

240.71

6.14

246.8 .5
ACTIVITY PERCENTAGE BY DISPOSAL SITE

(Percentages $<.1 \%$ are not displayed)

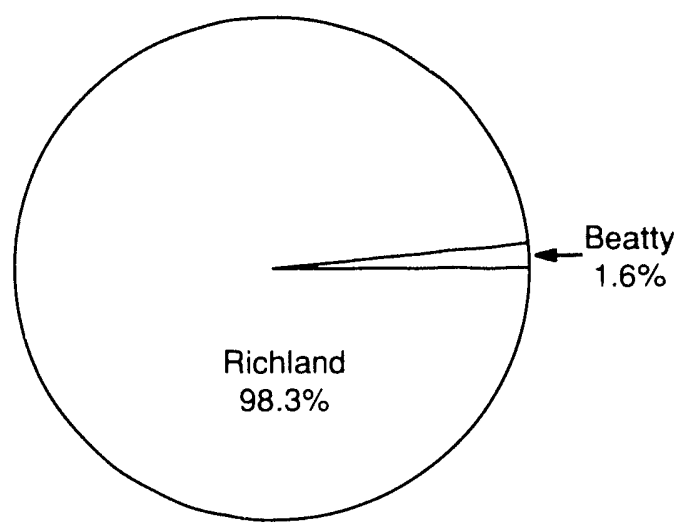

Volume Percentage by Waste Class for Direct Shipments and Total Non-Direct Volume Percentage (Percentages <. $1 \%$ are not displayed)

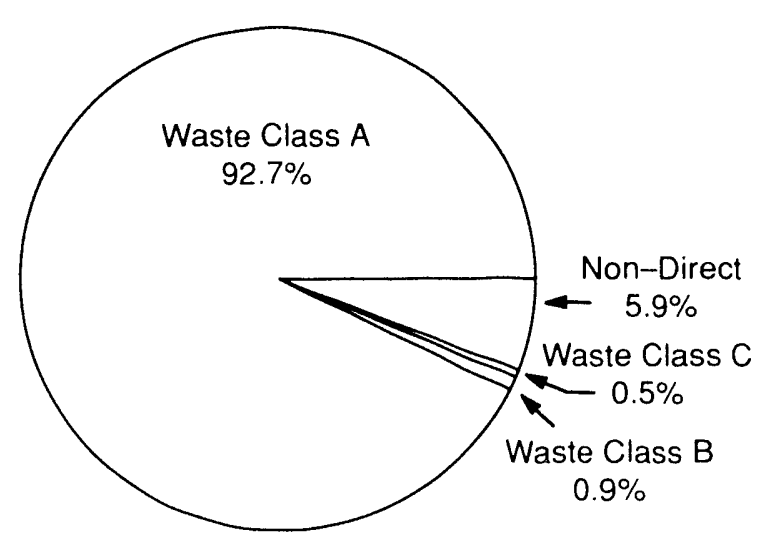




\section{CALIFORNIA \\ Low-Level Radioactive Wastes Received at Commercial Disposal Sites}

\begin{tabular}{l} 
Generator \\
Category \\
\hline
\end{tabular}

Academic

Government

Industrial

Medical

Utility

Total

$$
\begin{gathered}
\text { Volume } \\
\left(\mathrm{ft}^{3}\right)
\end{gathered}
$$

$11,447.95$

$5,793.74$

$21,538.12$

$3,595.78$

$13,580.95$

$55,956.54$
Activity

(curies)

35.18

31.48

$4,736.45$

25.92

708.65

$5,537.69$
VOLUME PERCENTAGE BY SOURCE

(Percentages <. $1 \%$ are not displayed)

Government

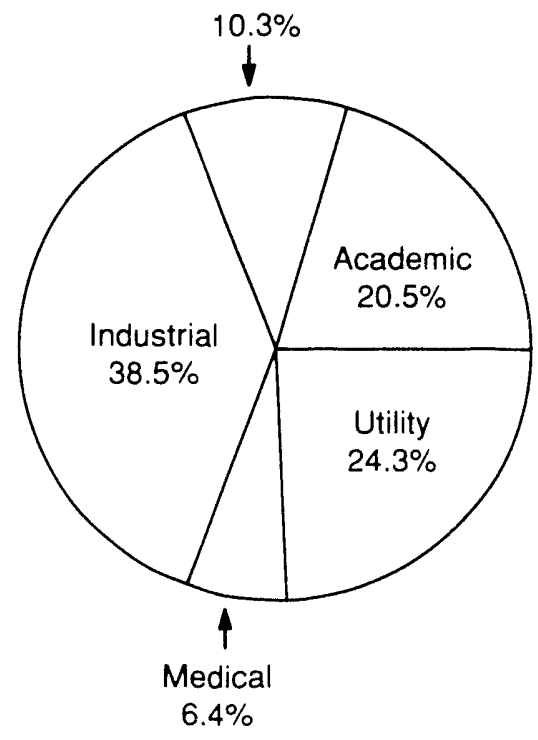

ACTIVITY PERCENTAGE BY SOURCE

(Percentages $<.1 \%$ are not displayed)

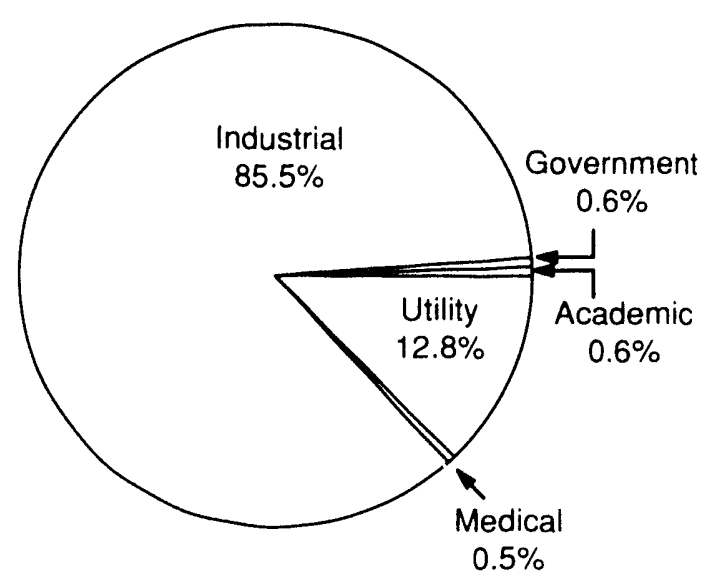




\section{CALIFORNIA \\ Low-Level Radioactive Wastes Received at Commercial Disposal Sites}

\begin{tabular}{lr}
\multicolumn{1}{c}{$\begin{array}{c}\text { Disposal } \\
\text { Site }\end{array}$} & \multicolumn{1}{c}{$\begin{array}{c}\text { Volume } \\
\left(\mathrm{ft}^{3}\right)\end{array}$} \\
Barnwell & \\
Beatty & $\begin{array}{r}4,325.89 \\
\text { Richland }\end{array}$ \\
Total & $29,721.40$ \\
& $21,909.25$ \\
\hline
\end{tabular}

\begin{tabular}{|c|}
\hline $\begin{array}{l}\text { Activity } \\
\text { (curies) }\end{array}$ \\
\hline 33.29 \\
\hline $3,827.92$ \\
\hline $1,676.47$ \\
\hline
\end{tabular}

VOLUME PERCENTAGE BY DISPOSAL SITE

(Percentages $<.1 \%$ are not displayed)

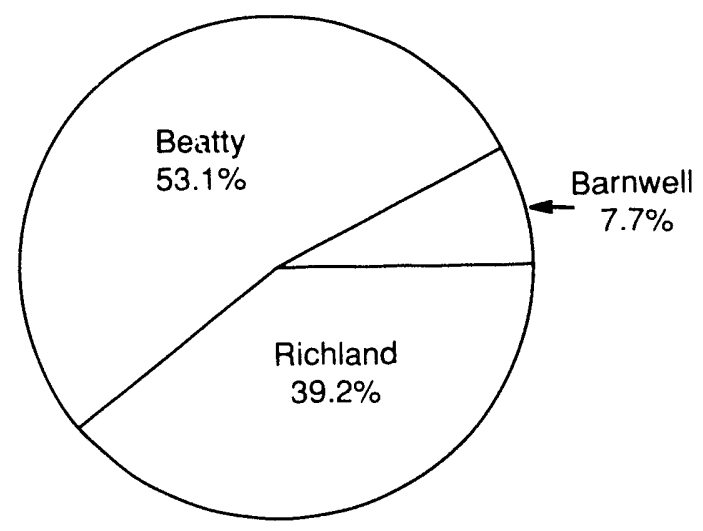

Direct Shipment

Waste Class

A

B

C

Total

Non-Direct Shipments

Grand Total

Total Direct Shipment

Activity (curies)

Total Non-Direct Shipment

Activity (curies)

Grand Total (curics) $\left(\mathrm{ft}^{3}\right)$

$20,795.00$

552.60

192.00

$21,539.60$

$34,416.94$

$55,956.54$
ACTIVITY PERCENTAGE BY DISPOSAL SITE

(Percentages <. $1 \%$ are not displayed)

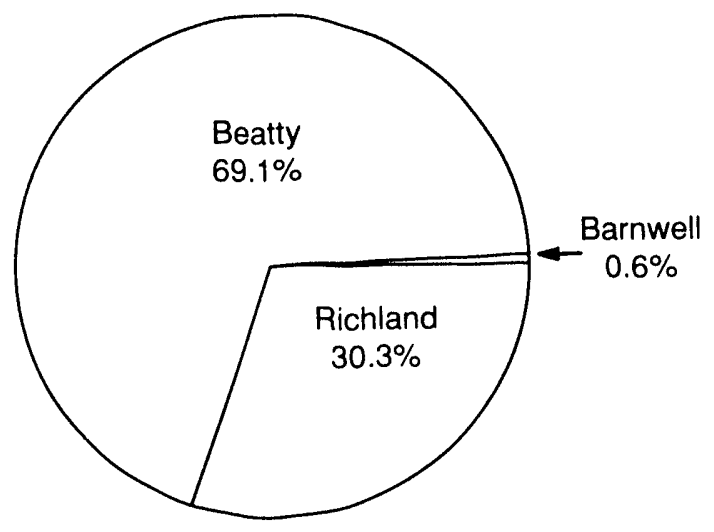

Volume Percentage by Waste Class for Direct Shipments and Total Non- Direct Volume Percentage

(Percentages <.1\% are not displayed)

Waste Class B

660.75

$4,876.94$

$5,537.69$

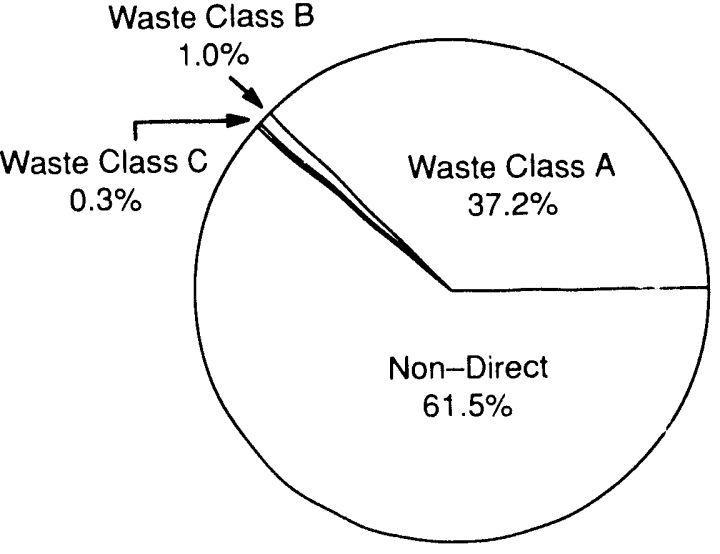




\section{NORTH DAKOTA \\ Low-Level Radioactive Wastes Received at Commercial Disposal Sites}

\section{Generator \\ Category}

Industrial

Medical

Total

$\begin{array}{r}\begin{array}{c}\text { Volume } \\ \left(\mathrm{ft}^{3}\right)\end{array} \\ \hline 25.40 \\ 7.50 \\ \hline\end{array}$

32.90
Activity (curies)

0.02
VOLUME PERCENTAGE BY SOURCE (Percentages <. $1 \%$ are not displayed)

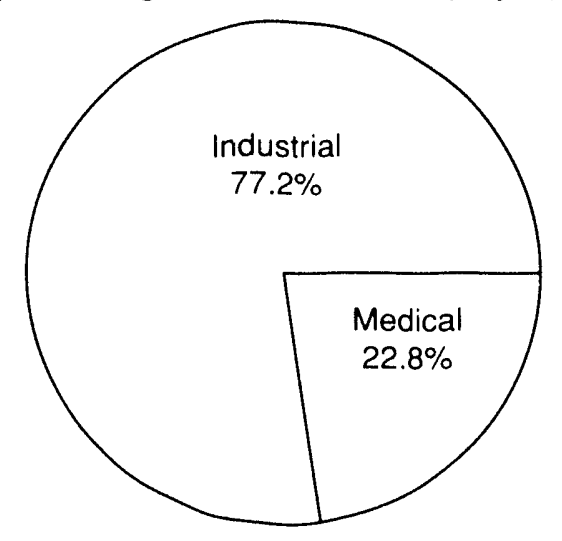

ACTIVITY PERCENTAGE BY SOURCE

(Percentages <. $1 \%$ are not displayed)

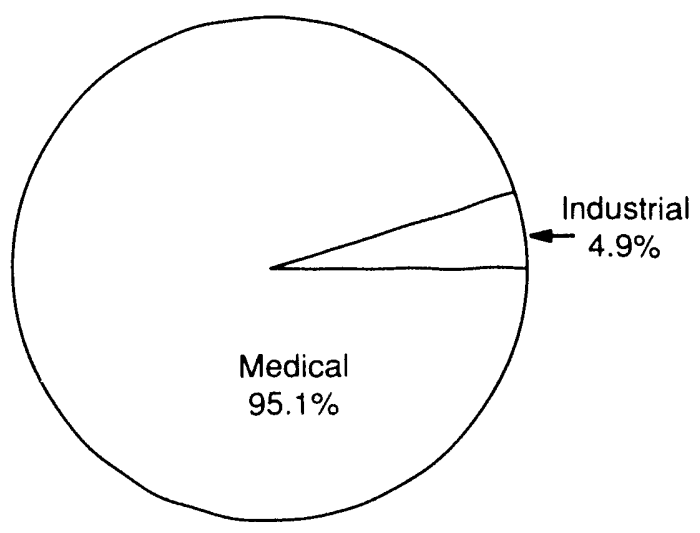




\section{NORTH DAKOTA \\ Low-Level Radioactive Wastes Received at Commercial Disposal Sites}

Disposal
Site

Beatty

Richland

Total

\author{
Volume \\ $\left(\mathrm{ft}^{3}\right)$
}

7.50

25.40

32.90
Activity

(curies)

0.02

$<.01$

0.02
VOLUME PERCENTAGE BY DISPOSAL SITE

(Percentages $<.1 \%$ are not displayed)

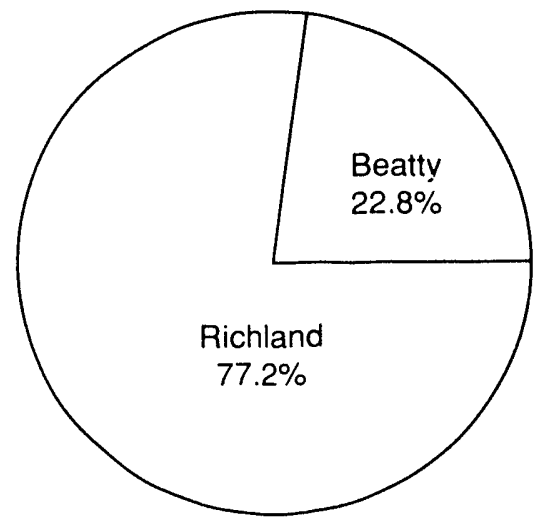

ACTIVITY PERCENTAGE BY DISPOSAL SITE

(Percentages <. $1 \%$ are not displayed)

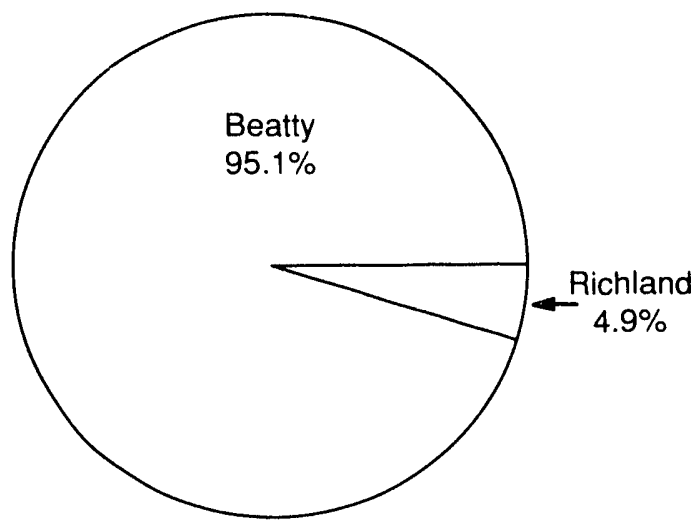

Volume Percentage by Waste Class for Direct Shipments and Total Non-Direct Volume Percentage

(Percentages $<.1 \%$ are not displayed)

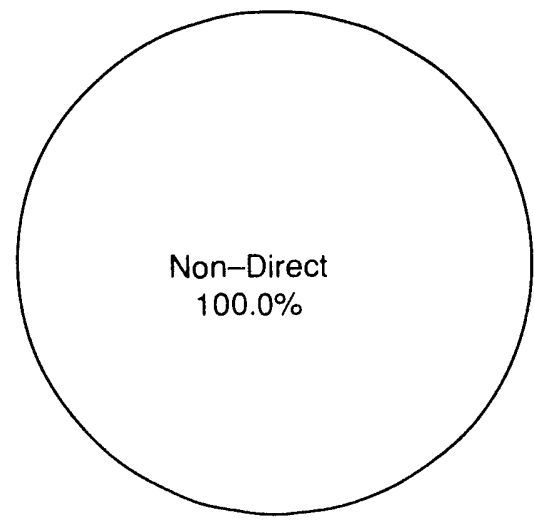




\section{SOUTH DAKOTA \\ Low-Level Radioactive Wastes Received at Commercial Disposal Sites}

\begin{tabular}{l} 
Generator \\
Category \\
\hline
\end{tabular}

Medical

Utility

Total

\begin{tabular}{c}
$\begin{array}{c}\text { Volume } \\
\left(\mathrm{ft}^{3}\right)\end{array}$ \\
\hline
\end{tabular}

0.54

$1,169.10$

$1,169.64$
Activity (curies)

$\begin{array}{r}0.05 \\ <.01 \\ \hline 0.05\end{array}$

VOLUME PERCENTAGE BY SOURCE (Percentages <. $1 \%$ are not displayed)

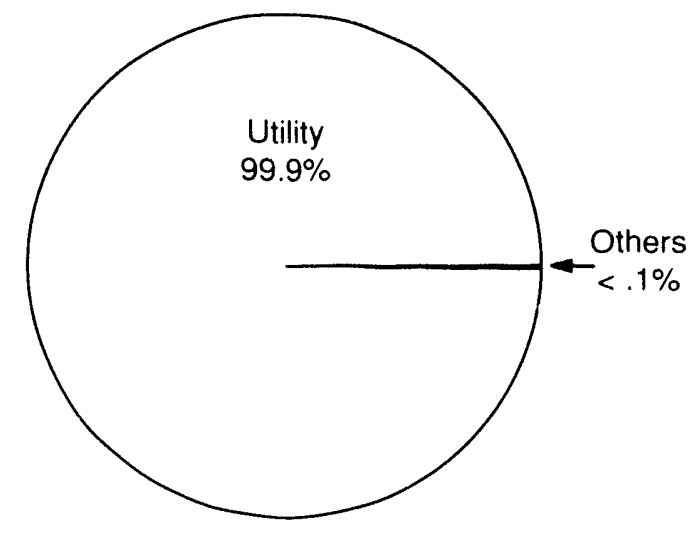

ACTIVITY PERCENTAGE BY SOURCE (Percentages $<.1 \%$ are not displayed)

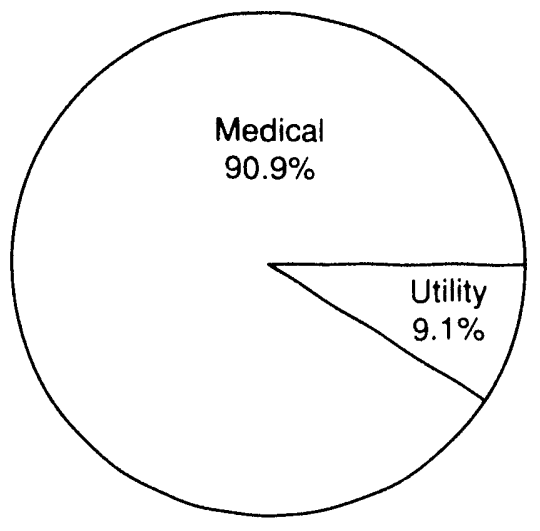




\section{SOUTH DAKOTA \\ Low-Level Radioactive Wastes Received at Commercial Disposal Sites}

Disposal
Site

Beatty

Richland

Total

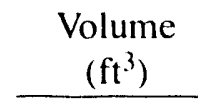

0.54

$1,169.10$

$1,169.64$
Activity

(curies)

0.05

$<.01$

0.05
VOLUME PERCENTAGE BY DISPOSAL SITE

(Percentages <. $1 \%$ are not displayed)

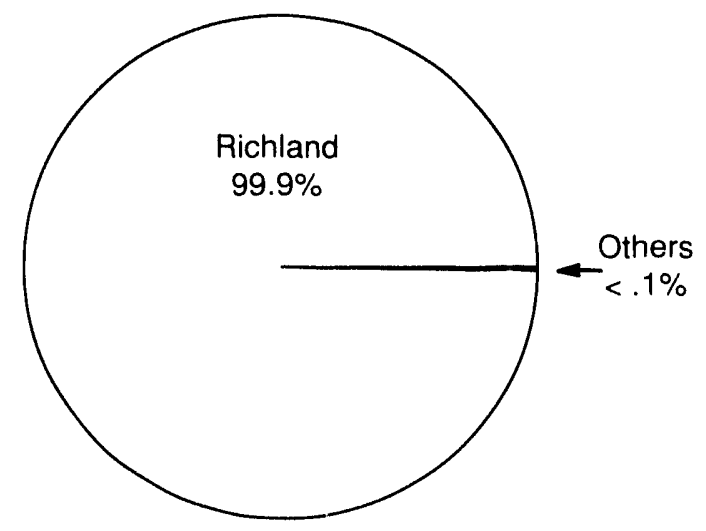

Direct Shipment

Waste Class

A

B

C

Total

Non-Direct Shipments

Grand Total

Total Direct Shipment

Activity (curies)

Total Non-Direct Shipment

Activity (curies)

Grand Total
$1,169.64$

$<.01$

Volume $\left(\mathrm{ft}^{3}\right)$

480.00

0.00

0.00

480.00

689.64

0.05

0.05
ACTIVITY PERCENTAGE BY DISPOSAL SITE

(Percentages <. $1 \%$ are not displayed)

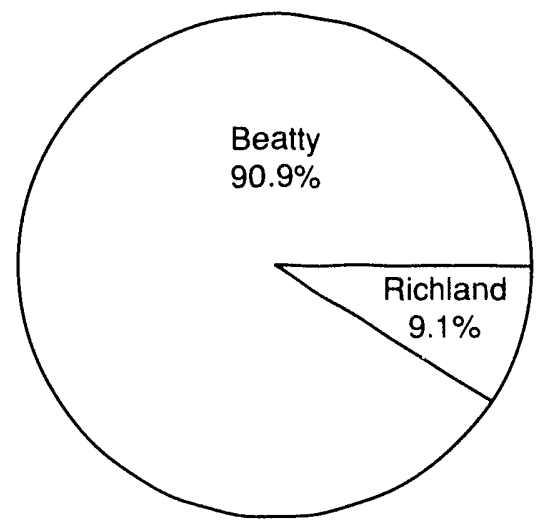

Volume Percentage by Waste Class for Direct Shipments and Total

Non-Direct Volume Percentage

(Percentages $<.1 \%$ are not displayed)

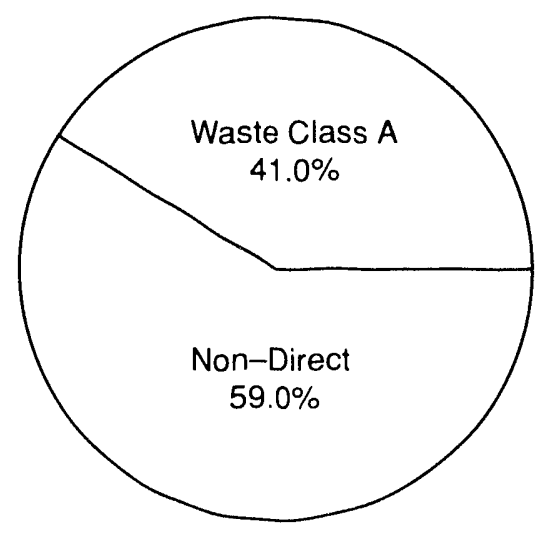


I

I

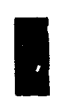

I

I

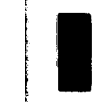

,

I

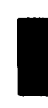

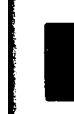

I

,

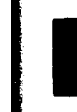

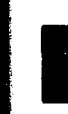

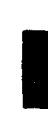

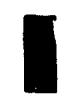

.

i 


\section{UNAFFILIATED \\ Low-Level Radioactive Wastes Received at Commercial Disposal Sites}

\begin{tabular}{l}
\multicolumn{1}{c}{ States } \\
\hline Army Out U.S. \\
Dis Of Columbia \\
Maine \\
Massachusetts \\
New Hampshire \\
New York \\
Rhode Island \\
Texas \\
Total
\end{tabular}

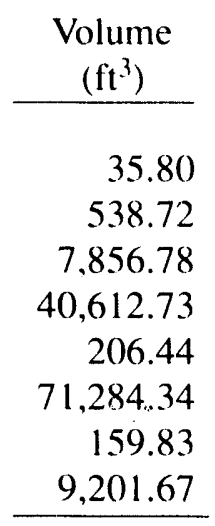

$129,896.31$
Activity

(curies)

0.03

5.13

193.25

86.641 .44

0.08

$12,701.84$

0.25

$1,650.32$

$101,192.34$ 


\section{ARMY OUT U.S. \\ Low-Level Radioactive Wastes Received at Commercial Disposal Sites}

\section{Generator \\ Categorv}

Government

Medical

Total

Volume
$\left(\mathrm{ft}^{3}\right)$

26.00

9.80

35.80
Activity

(curies)

$$
<.01
$$

0.03

0.03

VOLUME PERCENTAGE BY SOURCE

ACTIVITY PERCENTAGE BY SOURCE

(Percentages <. $1 \%$ are not displayed)

(Percentages $<.1 \%$ are not displayed)
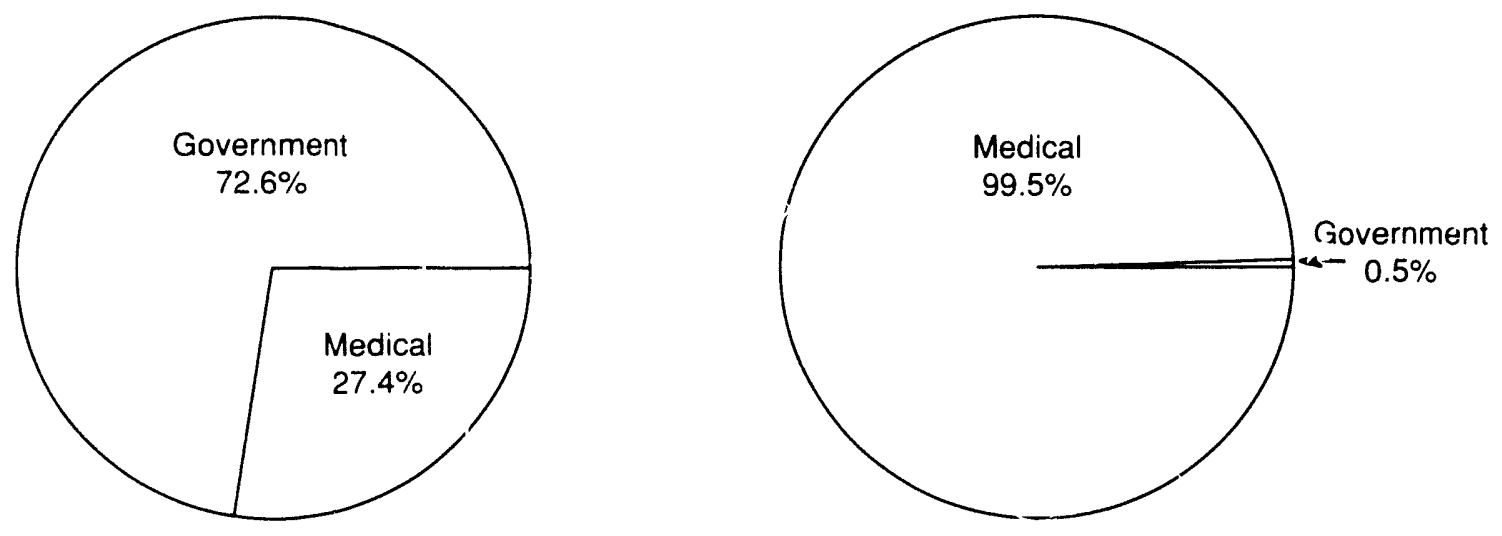


\section{ARMY OUT U.S. \\ Low-Level Radioactive Wastes Received at Commercial Disposal Sites}

\begin{tabular}{c} 
Disposal \\
Site \\
\hline
\end{tabular}

Barnwell

Total

\author{
Volume \\ $\left(\mathrm{ft}^{3}\right)$
}

35.80

35.80
Activity

(curies)

0.03

0.03
VOLUME PERCENTAGE BY DISPOSAL SITE

(Percentages <.1\% are not displayed)

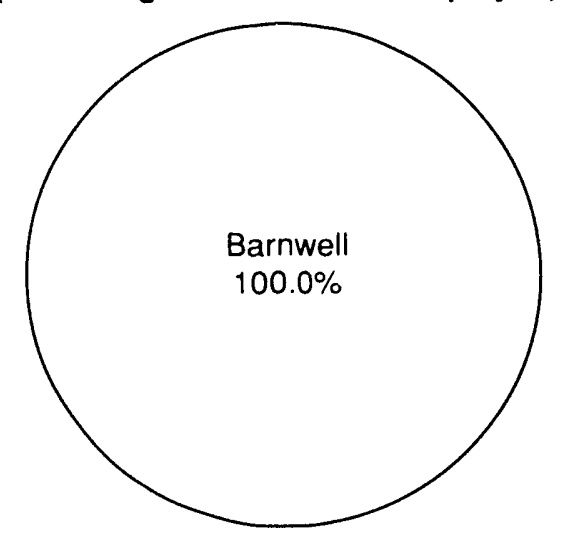

ACTIVITY PERCENTAGE BY DISPOSAL SITE

(Percentages <. $1 \%$ are not displayed)

$$
\begin{aligned}
& \text { Volume Percentage by Waste Class } \\
& \text { for Direct Shipments and Total } \\
& \text { Non-Direct Volume Percentage } \\
& \text { (Percentages }<.1 \% \text { are not displayed) }
\end{aligned}
$$

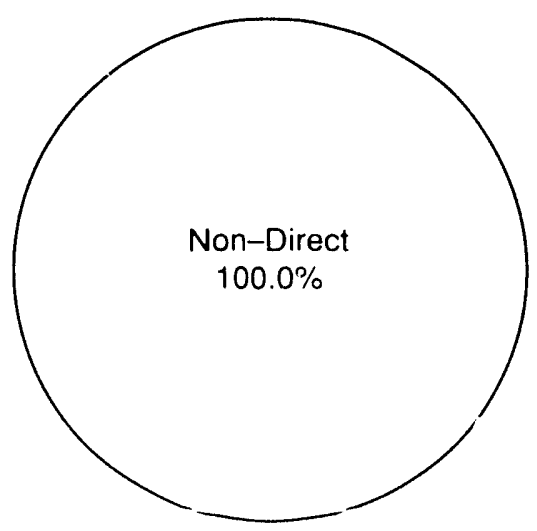




\section{DISTRICT OF COLUMËIA \\ Low-Level Radioactive Wastes Received at Commercial Disposal Sites}

\begin{tabular}{l} 
Generator \\
Category \\
\hline
\end{tabular}

Academic

Government

Industrial

Medical

Total
Volume

$\left(\mathrm{ft}^{3}\right)$

70.42

90.94

86.42

290.94

538.72
Aclivity

(curies)

0.14

4.25

0.28

0.46

5.13

VOLUME PERCENTAGE BY SOURCE (Percentages <. $1 \%$ are not displayed)

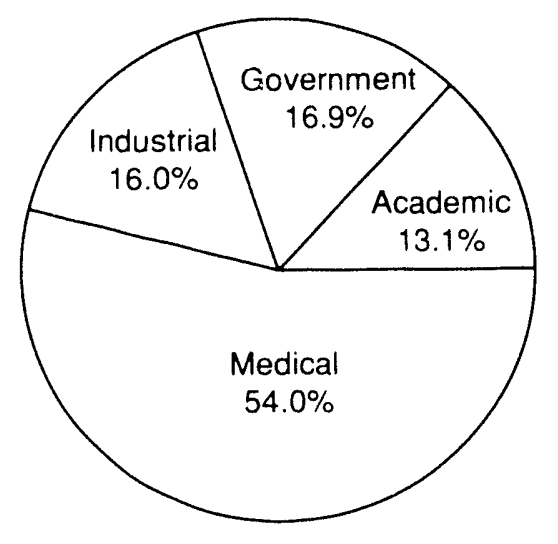

ACTIVITY PERCENTAGE BY SOURCE (Percentages <. $1 \%$ are not displayed)

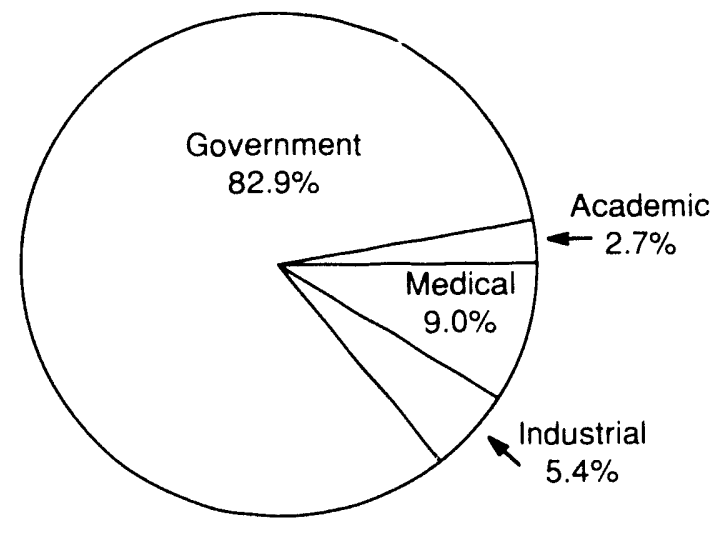




\section{DISTRICT OF COLUMBIA \\ Low-Level Radioactive Wastes Received at Commercial Disposal Sites}

\section{Disposal \\ Site}

Beatty

Richland

Total

\section{Volume}

$\left(\mathrm{ft}^{3}\right)$

530.62

8.10

538.72
Activity

(curies)

5.13

$<.01$

5.13
VOLUME PERCENTAGE BY DISPOSAL SITE
(Percentages $<.1 \%$ are not displayed)

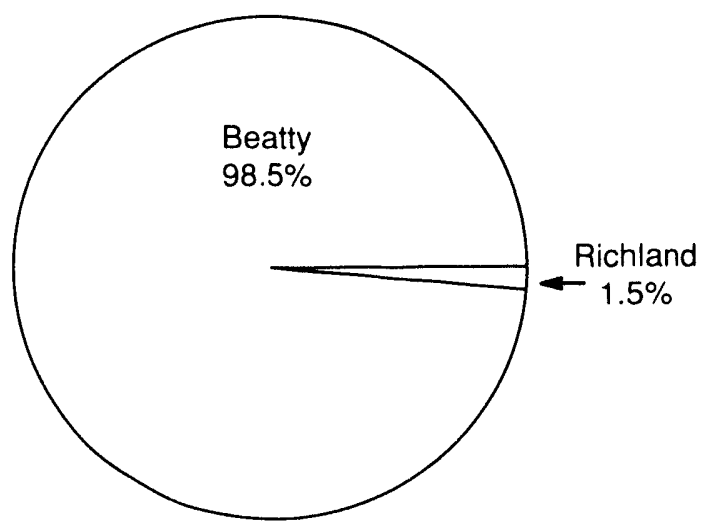

ACTIVITY PERCENTAGE BY DISPOSAL SITE (Percentages <. $1 \%$ are not displayed)

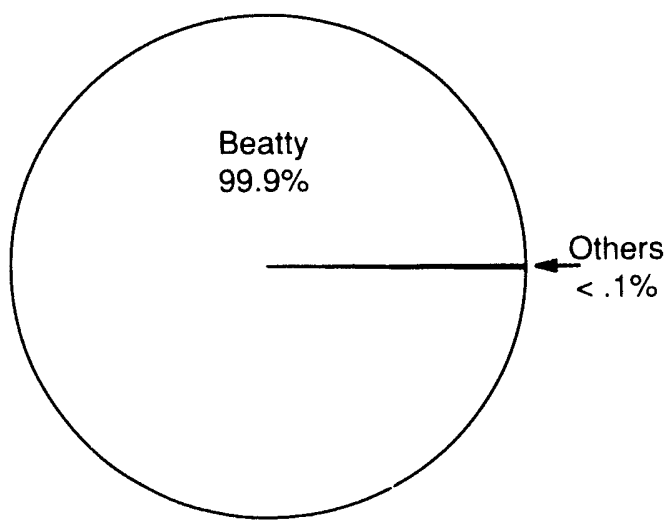

Volume Percentage by Waste Class

for Direct Shipments and Total

Non-Direct Volume Percentage

(Percentages <. $1 \%$ are not displayed)

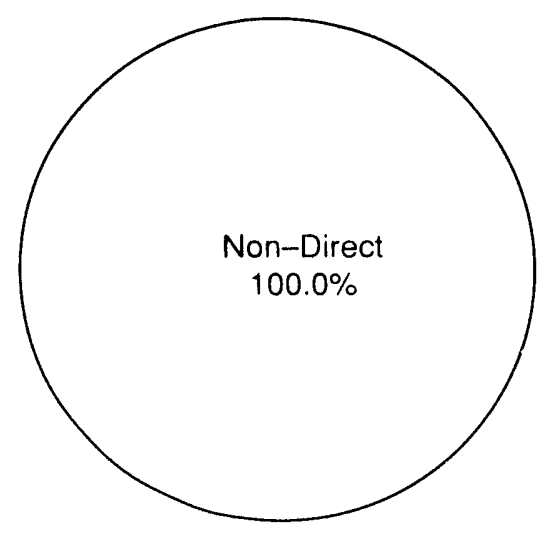




\section{MAINE \\ Low-Level Radioactive Wastes Received at Commercial Disposal Sites}

\begin{tabular}{l} 
Generator \\
Category \\
\hline
\end{tabular}

Government

Industrial

Utility

Total

\begin{tabular}{c}
$\begin{array}{c}\text { Volume } \\
\left(\mathrm{ft}^{3}\right)\end{array}$ \\
\hline
\end{tabular}

993.30

105.98

$6,757.50$

$7,856.78$
Activity

(curies)

$$
7.46
$$

185.13

193.25
VOLUME PERCENTAGE BY SOURCE

(Percentages <.1\% are not displayed)

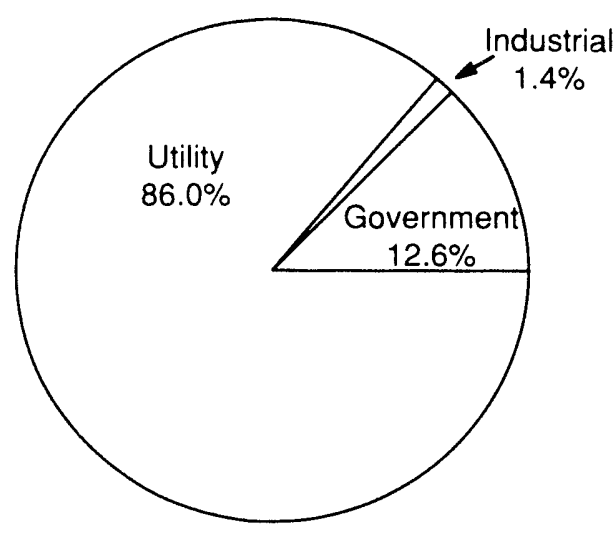

ACTIVITY PERCENTAGE BY SOURCE

(Percentages $<.1 \%$ are not displayed)

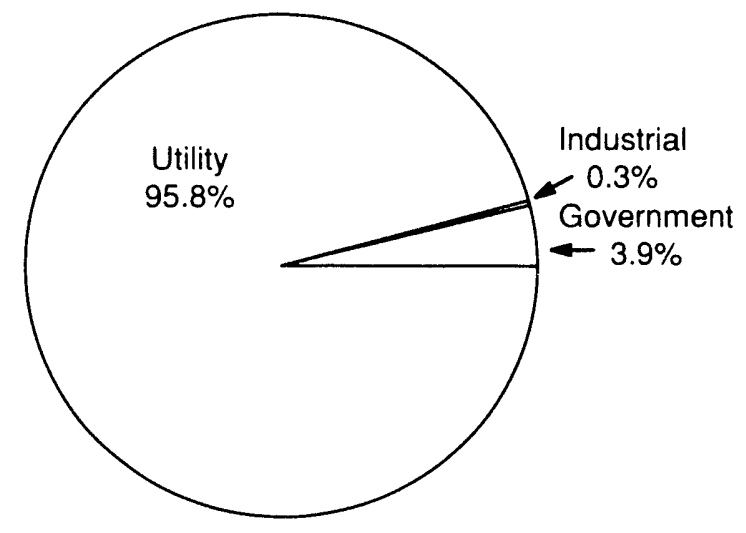




\section{MAINE \\ Low-Level Radioactive Wastes Received at Commercial Disposal Sites}

\begin{tabular}{c}
$\begin{array}{c}\text { Disposal } \\
\text { Site }\end{array}$ \\
\hline
\end{tabular}

Barnwell

Beatty

Richland

Total

$\begin{array}{r}\begin{array}{c}\text { Volume } \\ \left(\mathrm{ft}^{3}\right)\end{array} \\ \hline 6,768.50 \\ 1,024.48 \\ 63.80 \\ \hline 7,856.78\end{array}$

Activity

(curies)

192.50

0.61

0.14

193.25

\section{VOLUME PERCENTAGE BY DISPOSAL SITE (Percentages $<.1 \%$ are not displayed)}

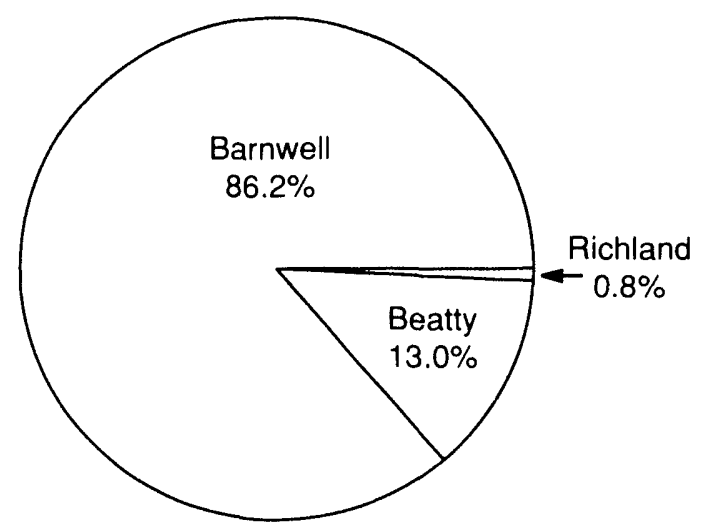

\section{ACTIVITY PERCENTAGE BY DISPOSAL SITE (Percentages <.1\% are not displayed)}

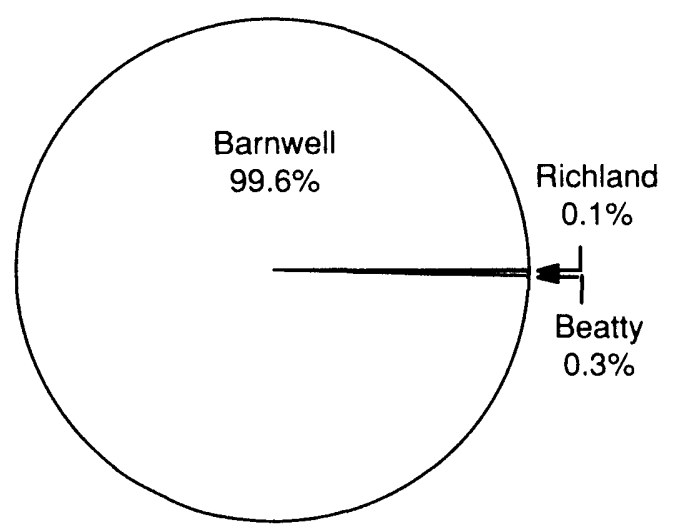

Volume Percentage by Waste Class for Direct Shipments and Total Non-Direct Volume Percentage (Percentages <.1\% are not displayed)
Direct Shipment Waste Class
A
B
C

Total

Non-Direct Shipments

Grand Total

Total Direct Shipment

Activity (curies)

Total Non-Direct Shipment

Activity (curies)

Grand Total

\section{Volume} $\left(\mathrm{ft}^{3}\right)$

$3,160.30$

354.50

28.00

$3,542.80$

$4,313.98$

$7,856.78$

180.21

13.04

193.25 


\section{MASSACHUSETTS \\ Low-Level Radioactive Wastes Received at Commercial Disposal Sites}

\begin{tabular}{l}
$\begin{array}{l}\text { Generator } \\
\text { Category }\end{array}$ \\
\hline
\end{tabular}

Academic

Government

Industrial

Medical

Utility

Total

\author{
Volume \\ $\left(\mathrm{ft}^{3}\right)$
}

539.78

296.40

$19,623.94$

558.92

$19,593.69$

$40,612.73$
Activity

(curies)

7.06

26.53

85.771 .11

3.58

833.15

$86,641.44$
VOLUME PERCENTAGE BY SOURCE

(Percentages <. $1 \%$ are not displayed)

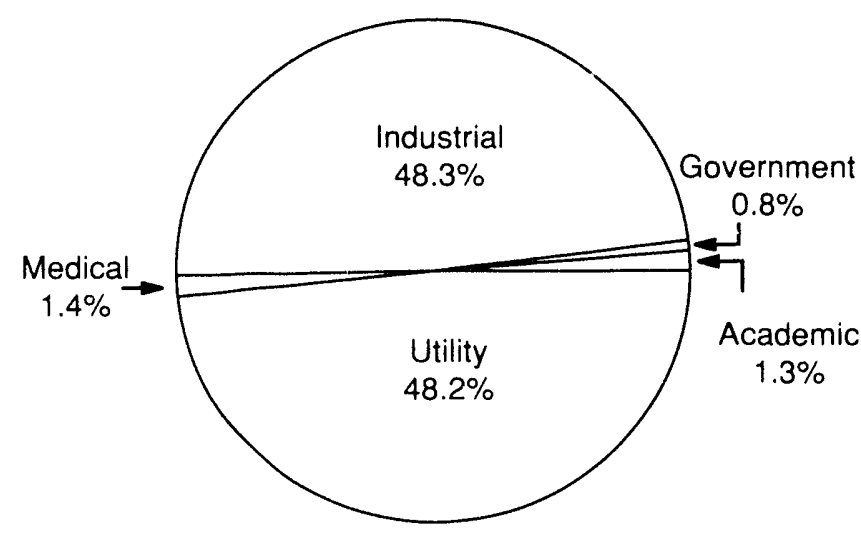

ACTIVITY PERCENTAGE BY SOURCE

(Percentages <. $1 \%$ are not displayed)

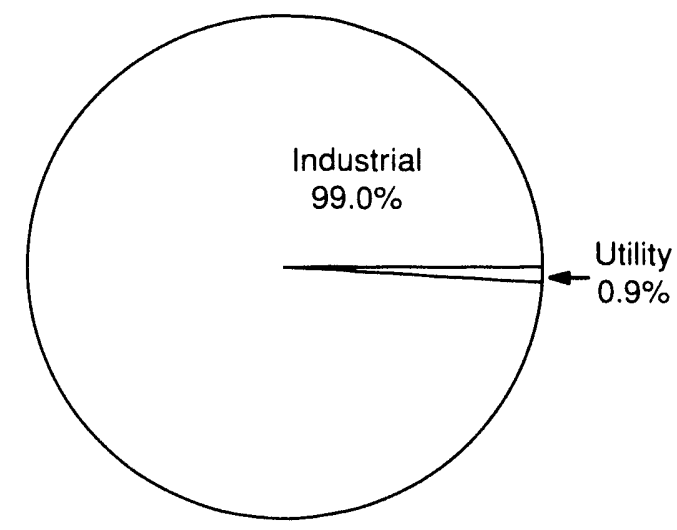




\section{MASSACHUSETTS \\ Low-Level Radioactive Wastes Received at Commercial Disposal Sites}

\begin{tabular}{c} 
Disposal \\
Site \\
\hline
\end{tabular}

Barnwell

Beatty

Richland

Total

\author{
Volume \\ $\left(\mathrm{ft}^{3}\right)$ \\ $25,339.15$ \\ $6,694.76$ \\ $8,578.82$
}

$40,612.73$
Activity

(curies)

928.24

109.11

$85,604.08$

$86,641.44$
VOLUME PERCENTAGE BY DISPOSAL SITE (Percentages $<.1 \%$ are not displayed)

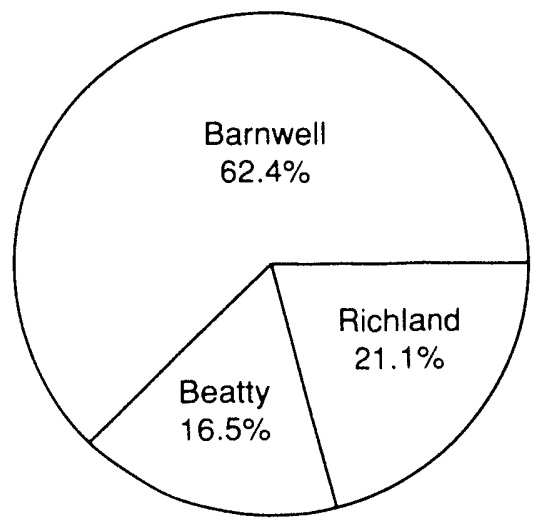

ACTIVITY PERCENTAGE BY DISPOSAL SITE

(Percentages $<.1 \%$ are not displayed)

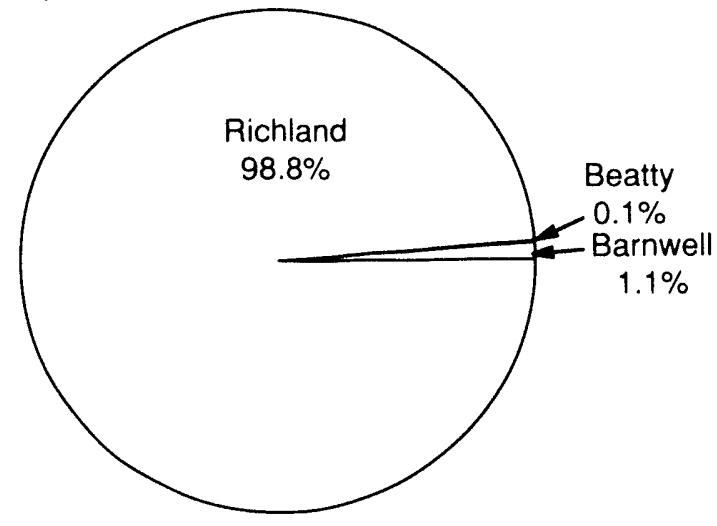

Volume Percentage by Waste Class for Direct Shipments and Total Non-Direct Volume Percentage (Percentages $<.1 \%$ are not displayed)
Direct Shipment

Waste Class

\section{A}

B

C

Total

Non-Direct Shipments

Grand Total

Total Direct Shipment

Activity (curies)

Total Non-Direct Shipment

Activity (curies)

Grand Total

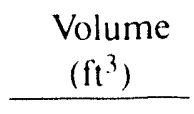

$21,939.70$

491.10

240.60

$22,671.40$

$17,941.33$

$40,612.73$

86.509 .65

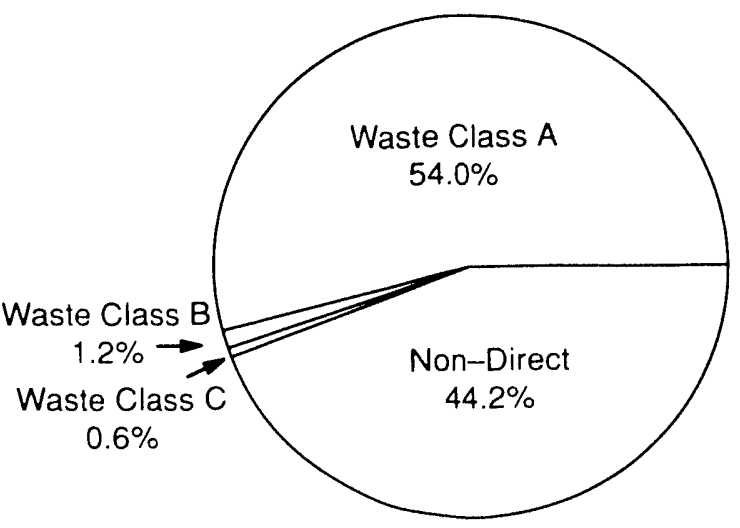

131.78

$86,641.44$ 


\section{NEW HAMPSHIRE \\ Low-Level Radioactive Wastes Received at Commercial Disposal Sites}

\begin{tabular}{l} 
Generator \\
Category \\
\hline
\end{tabular}

Industrial

Total
Volume

$\left(\mathrm{ft}^{3}\right)$

206.44

206.44
Activity

(curies)

0.08

0.08
VOLUME PERCENTAGE BY SOURCE (Percentages <. $1 \%$ are not displayed)

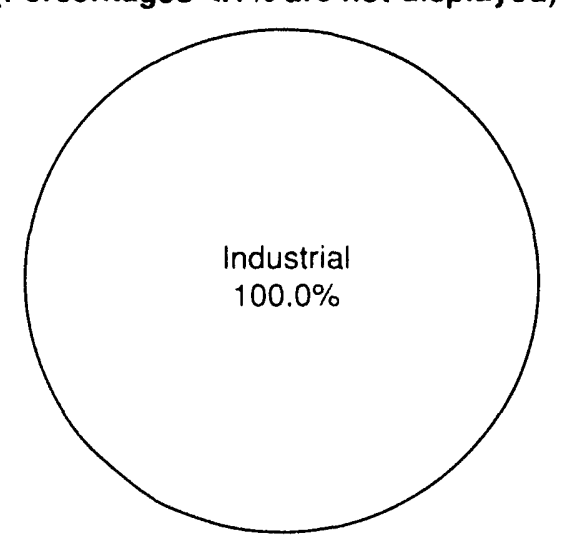

ACTIVITY PERCENTAGE BY SOURCE

(Percentages $<.1 \%$ are not displayed)

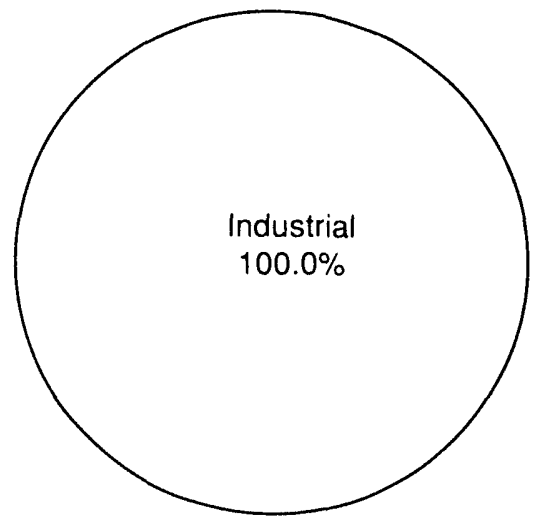




\section{NEW HAMPSHIRE \\ Low-Level Radioactive Wastes Received at Commercial Disposal Sites}

\begin{tabular}{c} 
Disposal \\
Site \\
\hline
\end{tabular}

Beatty

Total
Volume

$\left(\mathrm{ft}^{3}\right)$

206.44

206.44
Activity

(curies)

0.08
VOLUME PERCENTAGE BY DISPOSAL SITE

(Percentages $<.1 \%$ are not displayed)

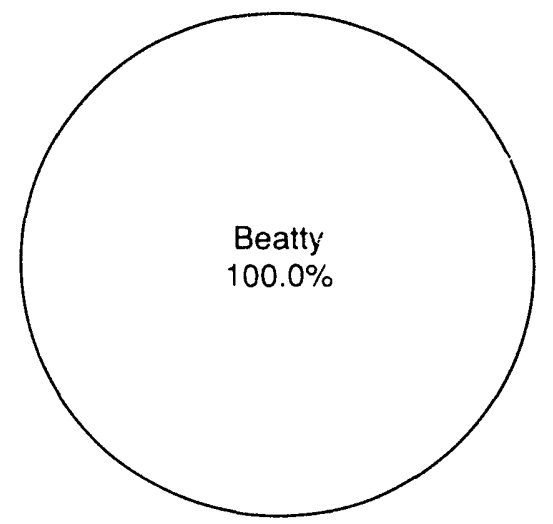

ACTIVITY PERCENTAGE BY DISPOSAL SITE

(Percentages <. $1 \%$ are not displayed)

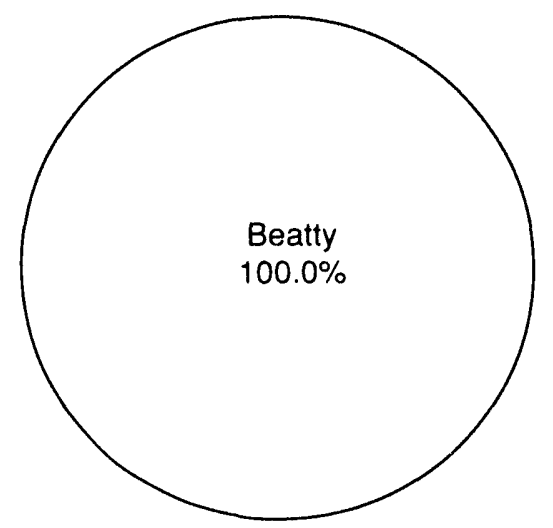

Volume Percentage by Waste Class

for Direct Shipments and Total

Non-Direct Volume Percentage

(Percentages <. $1 \%$ are not displayed)

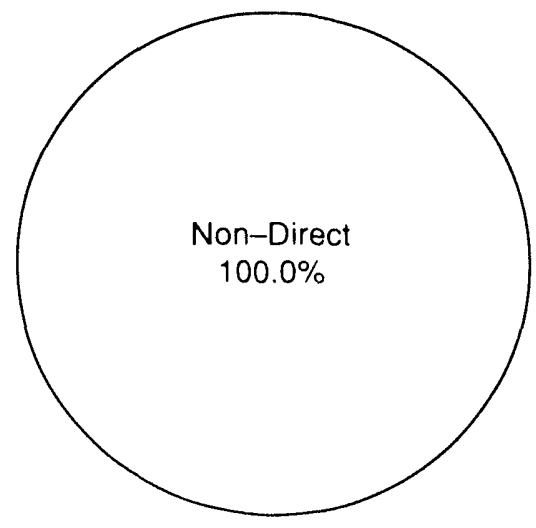




\section{NEW YORK \\ Low-Level Radioactive Wastes Received at Commercial Disposal Sites}

\begin{tabular}{lrr}
$\begin{array}{c}\text { Generator } \\
\text { Category }\end{array}$ & $\begin{array}{c}\text { Volume } \\
\left(\mathrm{ft}^{3}{ }^{3}\right)\end{array}$ & $\begin{array}{c}\text { Activity } \\
\text { (curies) }\end{array}$ \\
\cline { 1 - 1 } Academic & $2,259.82$ & 16.48 \\
Government & 12.68 & 160.44 \\
Industrial & $9,761.87$ & $7,355.93$ \\
Medical & $7,954.32$ & 9.07 \\
Utility & $51,295.65$ & $5,159.92$ \\
\hline Total & $71,284.34$ & $12,701.84$
\end{tabular}
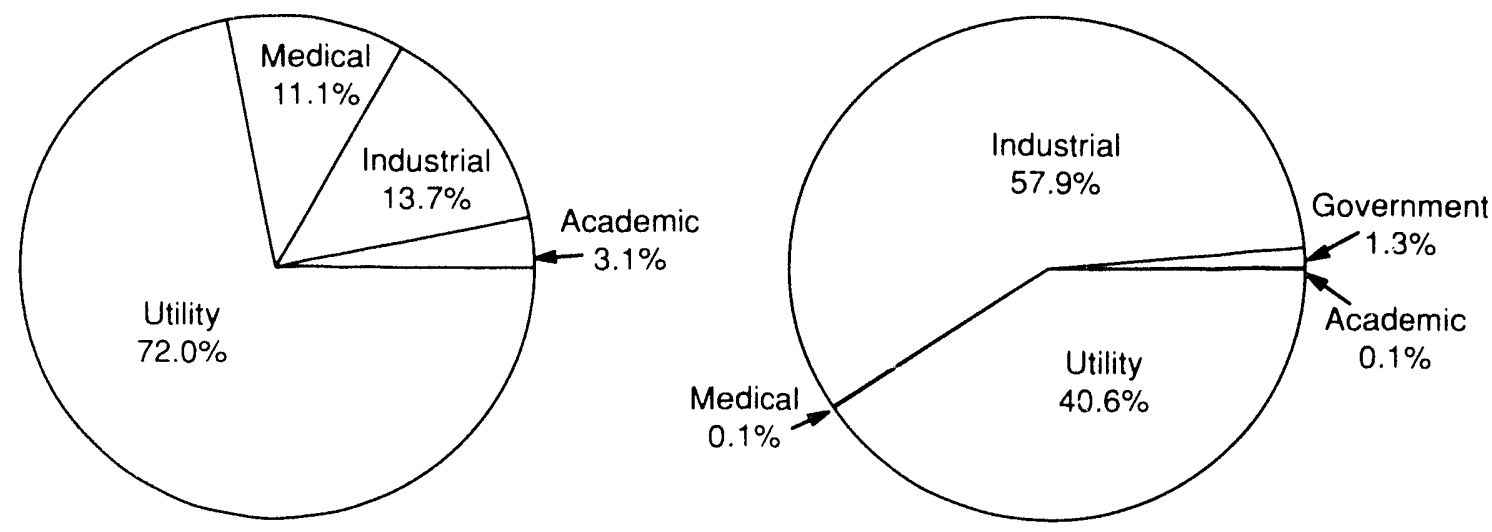


\section{NEW YORK \\ Low-Level Radioactive Wastes Received at Commercial Disposal Sites}

Disposal
Site

Barnwell

Beatty

Richland

Total
Volume

$\left(\mathrm{ft}^{3}\right)$

$52,615.11$

$1,116.12$

$17,553.11$

$71,284.34$
Activity

(curies)

$12,653.87$

9.87

38.10

$12,701.84$ VOLUME PERCENTAGE BY DISPOSAL SITE
(Percentages $<.1 \%$ are not displayed)

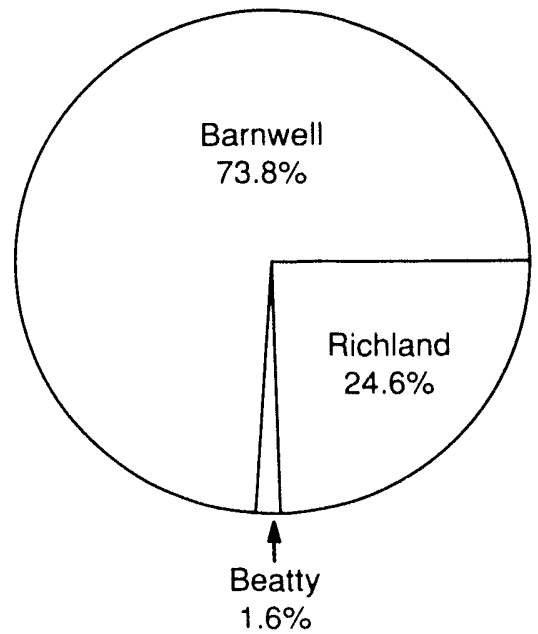

ACTIVITY PERCENTAGE BY DISPOSAL SITE (Percentages <. $1 \%$ are not displayed)

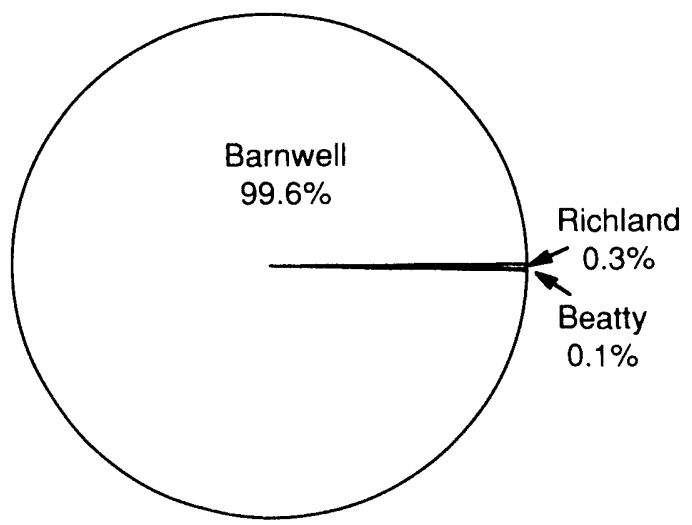

Volume Percentage by Waste Class for Direct Shipments and Total Non-Direct Volume Percentage (Percentages <. $1 \%$ are not displayed)
Direct Shipment

Waste Class

A

B

C

Total

Non-Direct Shipments

Grand Total

Total Direct Shipment

Activity (curies)

Total Non-Direct Shipment

Activity (curies)

Grand Total
Volume

$\left(\mathrm{ft}^{3}\right)$

$35,974.10$

$3,699.58$

163.50

$39,837.18$

$31,447.16$

$71,284.34$

$12,417.24$

284.60

$12,7(01.84$ 


\section{RHODE ISLAND \\ Low-Level Radioactive Wastes Received at Commercial Disposal Sites}

\section{Generator \\ Category}

Academic

Medical

Total

Volume
$\left(\mathrm{ft}^{3}\right)$

17.43

142.40

159.83
Activity

(curies)

0.04

0.21

0.25
VOLUME PERCENTAGE BY SOURCE (Percentages <. $1 \%$ are not displayed)

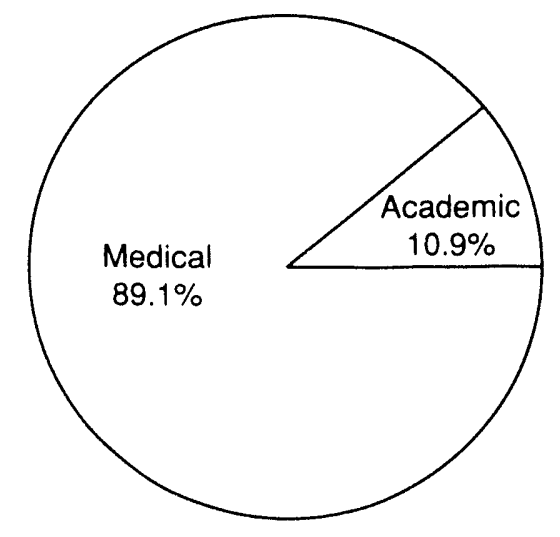

ACTIVITY PERCENTAGE BY SOURCE (Percentages <. $1 \%$ are not displayed)

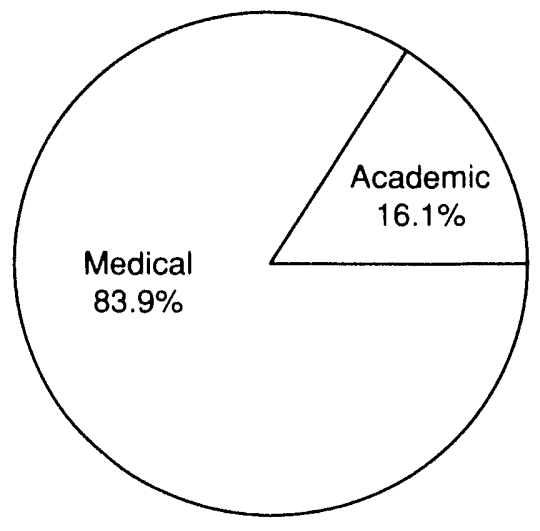




\section{RHODE ISLAND \\ Low-Level Radioactive Wastes Received at Commercial Disposal Sites}

\begin{tabular}{l} 
Disposal \\
Site \\
\hline
\end{tabular}

Beatty

Richland

Total
Volume

$\left(\mathrm{ft}^{3}\right)$

153.00

6.83

159.83
Activity

(curies)

0.22

0.03

0.25
VOLUME PERCENTAGE BY DISPOSAL SITE

(Percentages $<.1 \%$ are not displayed)

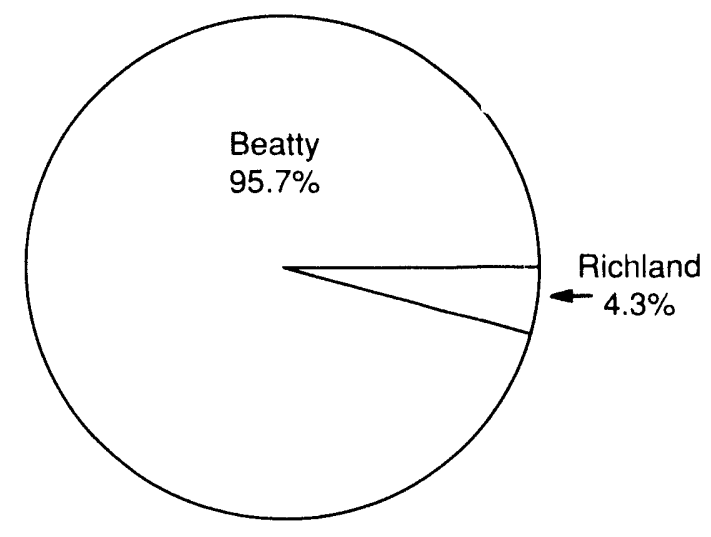

ACTIVITY PERCENTAGE BY DISPOSAL SITE

(Percentages $<.1 \%$ are not displayed)

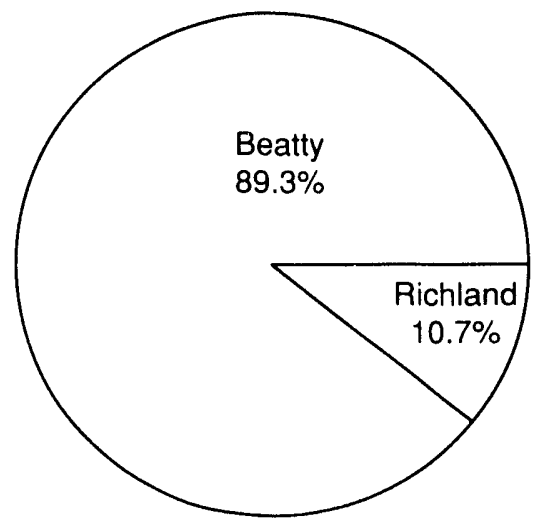

Volume Percentage by Wastc Class for Direct Shipments and Total

Non-Direct Volume Percentage

(Percentages <. $1 \%$ are not displayed)

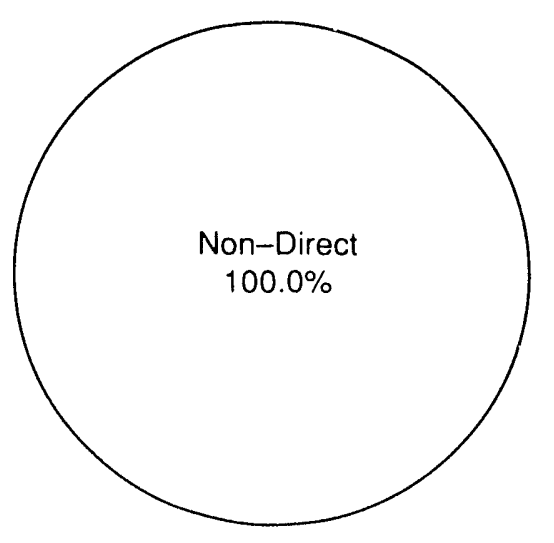




\section{TEXAS \\ Low-Level Radioactive Wastes Received at Commercial Disposal Sites}

\author{
Generator \\ Category \\ Academic \\ Government \\ Industrial \\ Medical \\ Utility
}

Total

\begin{tabular}{c}
$\begin{array}{c}\text { Volume } \\
\left(\mathrm{ft}^{3}\right)\end{array}$ \\
\hline 2.976 .22 \\
1.372 .95 \\
$2,503.63$ \\
267.07 \\
$2,081.80$ \\
\hline $9,201.67$
\end{tabular}

Activity

(curies)

$\begin{array}{r}5.44 \\ 500.28 \\ 1,129.69 \\ 1.05 \\ 13.86 \\ \hline 1,650.32\end{array}$

VOLUME PERCENTAGE BY SOURCE (Percentagles <.1\% are not displayed)

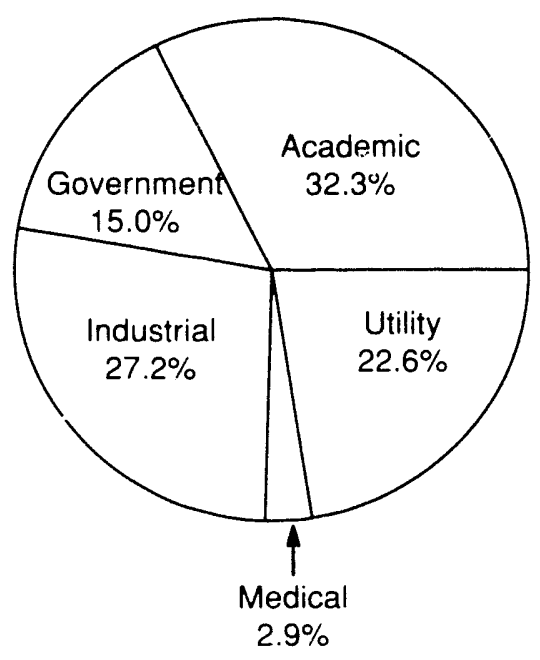

ACTIVITY PERCENTAGE BY SOURCE

(Percentages <. $1 \%$ are not displayed)

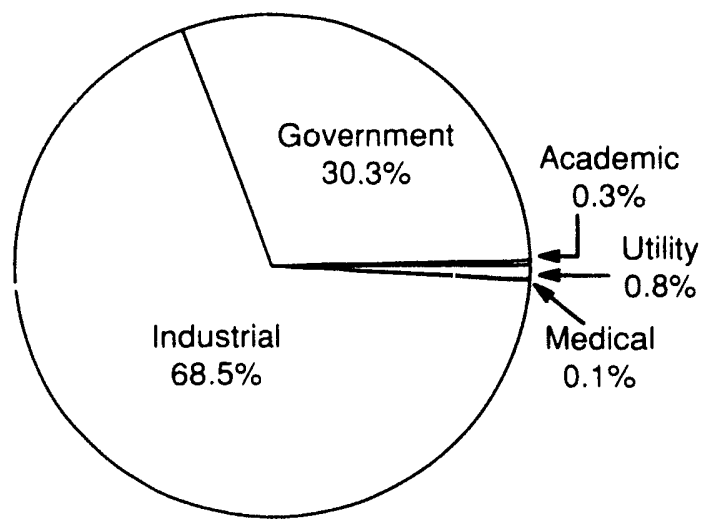




\section{TEXAS \\ Low-Level Radioactive Wastes Received at Commercial Disposal Sites}

Disposal
Site

Barnwell

Beatty

Richland

Total

\begin{tabular}{c}
$\begin{array}{c}\text { Volume } \\
\left(\mathrm{ft}^{3}\right)\end{array}$ \\
\hline $5,627.39$ \\
$2,031.04$ \\
$1,543.24$ \\
\hline $9,201.67$
\end{tabular}

Activity

(curies)

518.56

$1,128.17$

3.59

$1,650.32$
VOLUME PERCENTAGE BY DISPOSAL SITE

(Percentages <. $1 \%$ are not displayed)

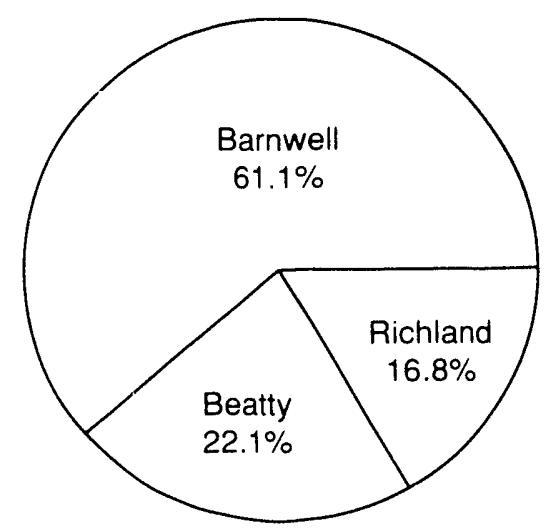

ACTIVITY PERCENTAGE BY DISPOSAL SITE
(Percentages $<.1 \%$ are not displayed)

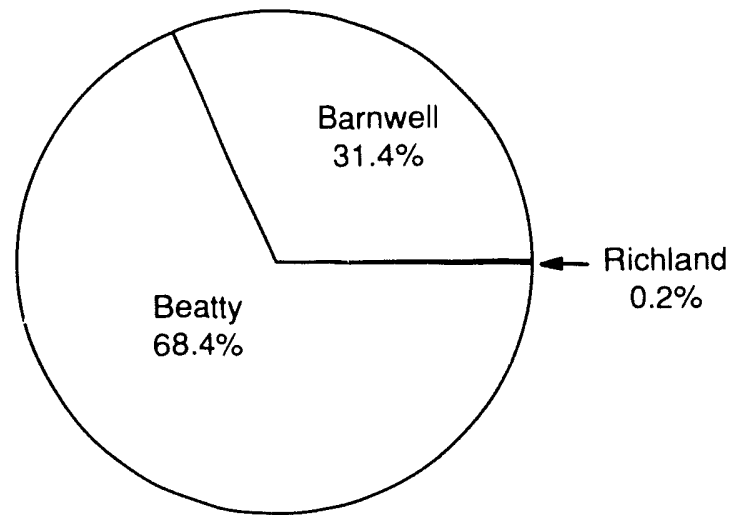

Direct Shipment

Waste Class

A

B

C

Total

Non-Direct Shipments

Grand Total

Volume

$\left(\mathrm{ft}^{3}\right)$

$2,278.20$

75.00

333.88

2.687 .08

$0,514.59$

9.201 .67

Total Direct Shipment

Activity (curies)

$1,139.48$

Total Non-Direct Shipment

Activit. (curies)

510.83

$1,650.32$

Grand Total
Volume Percentage by Waste Class for Direct Shipments and Total Non-Direct Volume Percentage (Percentages <. $1 \%$ are not displayed)

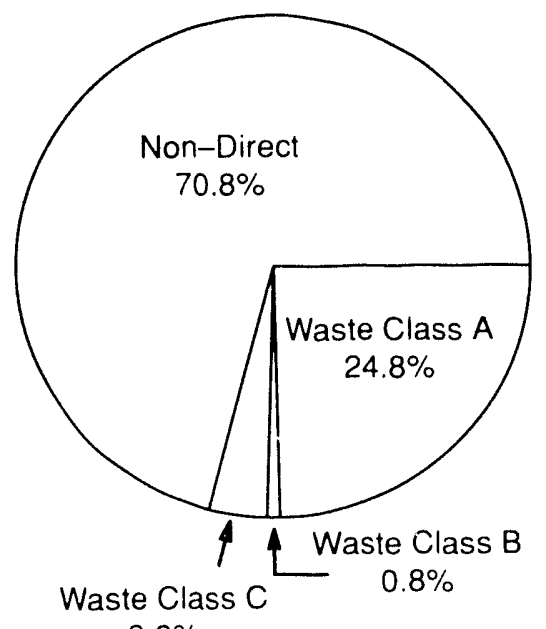
$3.6 \%$ 
I

I

I

I

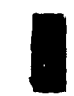

I

I

1

I

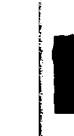

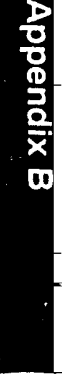




\section{APPENDIX B}

\section{WASTE VOLUME COMPARISON BY STATE}

AND

COMMERCIAL NUCLEAR POWER REACTORS IN THE UNITED STATES 
Page intentionally left blank. 
Table B-1. Waste Volume Comparison by State, 1986-1990, as Reported by Disposal Site Operators

\begin{tabular}{|c|c|c|c|c|c|}
\hline \multirow[b]{2}{*}{ State } & \multicolumn{5}{|c|}{$\begin{array}{r}\text { Volume } \\
\left(\mathrm{ft}^{3}\right)\end{array}$} \\
\hline & 1986 & 1987 & 1988 & 1989 & 1990 \\
\hline Alabama & 58,221 & 70,212 & 50,949 & 49,249 & 14,238 \\
\hline Alaska & 0 & 0 & 4 & 11 & 34 \\
\hline Arizona & 4,789 & 16,601 & 28345 & 31,756 & 27,751 \\
\hline Arkansas & 4,474 & 19,628 & 7.033 & 7,970 & 6,239 \\
\hline California & 114.011 & 99,304 & 83,157 & 117,966 & 55,957 \\
\hline Colorado & 1,073 & 3,586 & 2,104 & 8,754 & 2,309 \\
\hline Connecticut & 55,700 & 40,445 & 41,890 & 49,078 & 34,138 \\
\hline Delaware & 1,473 & 1,404 & 1,420 & 1,377 & 844 \\
\hline District of Columbia & 230 & 135 & 980 & 925 & 539 \\
\hline Florida & 60,109 & 46,079 & 31,027 & 35,283 & 24,587 \\
\hline Georgia & 48,275 & 29,615 & 38,940 & 44,697 & 55,287 \\
\hline Hawaii & 2,029 & 2,598 & 3,943 & 6,204 & 4,739 \\
\hline Idaho & 0 & 2 & 51 & 138 & 40 \\
\hline Illinois & 227,171 & 190,224 & 112,529 & 134,787 & 98,360 \\
\hline Indiana & 0 & 1,808 & 1,892 & 2,150 & 1,956 \\
\hline Iowa & 9,973 & 18.646 & 7,207 & 6,055 & 5,558 \\
\hline Kansas & 6,575 & 4,506 & 5,015 & 6,365 & 3,233 \\
\hline Kentucky & 3,790 & 176 & 2,122 & 10,047 & 4,616 \\
\hline Louisiana & 22,534 & 27,826 & 18,401 & 21,723 & 11,329 \\
\hline Maine & 8.324 & 5,013 & 6,338 & 15,633 & 7,857 \\
\hline Maryland & 18,856 & 19,637 & 30,604 & 39,996 & 17,038 \\
\hline Massachusetts & 66,695 & 55,023 & 51,273 & 56,527 & 40,613 \\
\hline Michigan & 36,234 & 35,284 & 27,136 & 43,111 & 36,039 \\
\hline Minnesota & 28,061 & 19,637 & 15,477 & 21,954 & 26,865 \\
\hline Mississippi & 18,625 & 14,119 & 18,173 & 10,996 & 5,981 \\
\hline Missouri & 24,497 & 22,858 & 11,912 & 18,803 & 19,610 \\
\hline Montana & 591 & 38 & 14 & 107 & 195 \\
\hline Nebraska & 20,342 & 17,409 & 12,822 & 16,699 & 15,559 \\
\hline Nevada & 0 & 15 & 668 & 308 & 313 \\
\hline New Hampshire & 2,109 & 394 & 169 & 27 & 206 \\
\hline New Jersey & 55,521 & 50,292 & 46,371 & 53,540 & 52,879 \\
\hline New Mexico & 0 & 990 & 304 & 1,369 & 1,844 \\
\hline New York & 106,547 & 70,409 & 73.069 & 96,893 & 71,284 \\
\hline North Carolina & 81,847 & 78,791 & 62,446 & 58,422 & 48.098 \\
\hline North Dakota & 0 & 3 & 0 & 105 & 33 \\
\hline Ohio & $16,(052$ & 18,662 & $22,58.5$ & 58,866 & 24,147 \\
\hline Oklahoma & 49,815 & 82,855 & 28,446 & 32,127 & 21,968 \\
\hline Oregon & 108,983 & 82,150 & 83.796 & 75,908 & $59,4.56$ \\
\hline Pennsylvania & 188.038 & 144.798 & 150,298 & 129,485 & 101,604 \\
\hline Puerto Rico & 0 & 0 & 0 & 0 & () \\
\hline Rhode Island & 1,210 & $1,04.5$ & 1,108 & 1,419 & 1.59 \\
\hline South Carolina & 121,134 & 118,060 & 95,168 & 96,695 & 46,927 \\
\hline
\end{tabular}


Table B-1. (continued)

\begin{tabular}{|c|c|c|c|c|c|}
\hline \multirow[b]{2}{*}{ State } & \multicolumn{5}{|c|}{$\begin{array}{r}\text { Volume } \\
\left(\mathrm{ft}^{3}\right)\end{array}$} \\
\hline & 1986 & 1987 & 1988 & 1989 & 1990 \\
\hline South Dakota & 8 & 0 & 0 & 1 & 1,170 \\
\hline Tennessee & 81,431 & 238,779 & 119,051 & 118.957 & 83,402 \\
\hline Texas & 4,376 & 69,201 & 12,064 & 22,158 & 9,202 \\
\hline Utah & 3,405 & 2,715 & 4,672 & 6,299 & 5,338 \\
\hline Vermont & 11,753 & 8,263 & 7,248 & 173 & () \\
\hline Virginia & 71,196 & 67,985 & 64,129 & 82,834 & 57,766 \\
\hline Washington & 52,987 & 38,244 & 35,896 & 26,409 & 26,141 \\
\hline West Virginia & 2 & 5 & 148 & 411 & 95 \\
\hline Wisconsin & 5,938 & 9,035 & 10,599 & 6,881 & 9,217 \\
\hline Wyoming & 0 & 0 & 0 & 1 & 15 \\
\hline Army out U.S. & $N / R$ & $N / R$ & 3 & 164 & 36 \\
\hline Total & $1,804,999$ & $1,844,504$ & $1,429,016$ & $1,627,813$ & $1,142,810$ \\
\hline
\end{tabular}


Table B-2. Commercial Nuclear Power Reactors in the United States, as of December 31, 1990a

\begin{tabular}{|c|c|c|c|}
\hline State Name & Facility Name & Units & Type \\
\hline \multirow[t]{2}{*}{ Alabama } & Browns Ferry Nuclear Power Station & $1,2 \& 3$ & $B W R^{b}$ \\
\hline & Joseph M. Farley Nuclear Plant & $1 \& 2$ & $\mathrm{PWR}^{\mathrm{c}}$ \\
\hline Arizona & Palo Verde Nuclear Generating Station & $1,2 \& 3$ & PWR \\
\hline Arkansas & Arkansas Nuclear One & $1 \& 2$ & PWR \\
\hline \multirow[t]{4}{*}{ California } & Diablo Canyon Nuclear Power Plant & $1 \& 2$ & PWR \\
\hline & Humboldt Bay Power Plant (closed 1976) & 3 & BWR \\
\hline & $\begin{array}{l}\text { Rancho Seco Nuclear Generating Station } \\
\text { (closed June 1989) }\end{array}$ & 1 & PWR \\
\hline & San Onofre Nuclear Generating Station & $1,2 \& 3$ & PWR \\
\hline Colorado & $\begin{array}{l}\text { Ft. St. Vrain Nuclear Generating Station } \\
\text { (closed August 1989) }\end{array}$ & 1 & HTGR $^{d}$ \\
\hline \multirow[t]{3}{*}{ Connecticut } & Haddam Neck Plant & 1 & PWR \\
\hline & Millstone Nuclear Power Station & 1 & BWR \\
\hline & Millstone Nuclear Power Station & $2 \& 3$ & PWR \\
\hline \multirow[t]{3}{*}{ Florida } & Crystal River Nuclear Plant & 3 & PWR \\
\hline & St. Lucie Plant & $1 \& 2$ & PWR \\
\hline & Turkey Point Plant & $3 \& 4$ & PWR \\
\hline \multirow[t]{2}{*}{ Georgia } & Edwin I. Hatch Nuclear Plant & $1 \& 2$ & BWR \\
\hline & Alvin W. Vogtle Nuclear Plant & $1 \& 2$ & PWR \\
\hline \multirow[t]{7}{*}{ Illinois } & Byron Nuclear Power Station & $1 \& 2$ & PWR \\
\hline & Clinton Power Station & 1 & BWR \\
\hline & $\begin{array}{l}\text { Dresden Nuclear Power Station (Unit } 1 \\
\text { closed 1978) }\end{array}$ & $1,2 \& 3$ & BWR \\
\hline & LaSalle County Station & $1 \& 2$ & BWR \\
\hline & Quad Cities Station & $1 \& 2$ & BWR \\
\hline & Zion Nuclear Plant & $1 \& 2$ & PWR \\
\hline & Braidwood Station & $1 \& 2$ & PWR \\
\hline Iowa & Duane Arnold Energy Center & 1 & BWR \\
\hline Kansas & Wolf Creek Generating Station & 1 & PWR \\
\hline \multirow[t]{2}{*}{ Louisiana } & River Bend Station & 1 & BWR \\
\hline & Waterford Generating Station & 3 & PWR \\
\hline
\end{tabular}


Table B-2. (continued)

\begin{tabular}{|c|c|c|c|}
\hline State Name & Facility Name & Units & Type \\
\hline Maine & Maine Yankee Atomic Power Plant & 1 & PWR \\
\hline Maryland & Calvert Cliffs Nuclear Power Plant & $1 \& 2$ & PWR \\
\hline Massachusetts & $\begin{array}{l}\text { Pilgrim Nuclear Power Station } \\
\text { Yankee Nuclear Power Station }\end{array}$ & $\begin{array}{l}1 \\
1\end{array}$ & $\begin{array}{l}\text { BWR } \\
\text { PWR }\end{array}$ \\
\hline Michigan & $\begin{array}{l}\text { Big Rock Point Nuclear Plant } \\
\text { Donald C. Cook Nuclear Power Plant } \\
\text { Fermi Atomic Power Plant } \\
\text { Palisades Nuclear Plant }\end{array}$ & $\begin{array}{l}1 \\
1 \& 2 \\
2 \\
1\end{array}$ & $\begin{array}{l}\text { BWR } \\
\text { PWR } \\
\text { BWR } \\
\text { PWR }\end{array}$ \\
\hline Minnesota & $\begin{array}{l}\text { Monticello Nuclear Generatirg Plant } \\
\text { Prairie Island Nuclear Generating Plant }\end{array}$ & $1 \& 2$ & $\begin{array}{l}\text { BWR } \\
\text { PWR }\end{array}$ \\
\hline Mississippi & Grand Gulf Nuclear Station & 1 & BWR \\
\hline Missouri & Callaway Plant & 1 & PWR \\
\hline Nebraska & $\begin{array}{l}\text { Cooper Nuclear Station } \\
\text { Fort Calhoun Station }\end{array}$ & $\begin{array}{l}1 \\
1\end{array}$ & $\begin{array}{l}\text { BWR } \\
\text { PWR }\end{array}$ \\
\hline New Hampshire & Seabrook Nuclear Station & 1 & PWR \\
\hline New Jersey & $\begin{array}{l}\text { Hope Creek Nuclear Generating Station } \\
\text { Oyster Creek Nuclear Power Plant } \\
\text { Salem Nuclear Generating Station }\end{array}$ & $\begin{array}{l}1 \\
1 \\
1 \& 2\end{array}$ & $\begin{array}{l}\text { BWR } \\
\text { BWR } \\
\text { PWR }\end{array}$ \\
\hline New York & $\begin{array}{l}\text { James A. FitzPatrick Nuclear Power Plant } \\
\text { Robert Emmett Ginna Nuclear Power Plant } \\
\text { Indian Point Station } \\
\text { Indian Point Station (Unit } 1 \text { closed 1974) } \\
\text { Nine Mile Point Nuclear Station } \\
\text { Shoreham Nuclear Power Station } \\
\text { (closed May 1989) }\end{array}$ & $\begin{array}{l}1 \\
1 \\
3 \\
1 \& 2 \\
1 \& 2 \\
1\end{array}$ & $\begin{array}{l}\text { BWR } \\
\text { PWR } \\
\text { PWR } \\
\text { PWR } \\
\text { BWR } \\
\text { BWR }\end{array}$ \\
\hline North Carolina & $\begin{array}{l}\text { Brunswick Steam Electric Plant } \\
\text { McGuire Nuclear Station } \\
\text { Shearon Harris Nuclear Power Plant }\end{array}$ & $\begin{array}{l}1 \& 2 \\
1 \& 2 \\
1\end{array}$ & $\begin{array}{l}\text { BWR } \\
\text { PWR } \\
\text { PWR }\end{array}$ \\
\hline Ohio & $\begin{array}{l}\text { Davis-Besse Nuclear Power Station } \\
\text { Perry Nuclear Power Plant }\end{array}$ & 1 & $\begin{array}{l}\text { PWR } \\
\text { BWR }\end{array}$ \\
\hline Oregon & Trojan Nuclear Plant & 1 & PWR \\
\hline
\end{tabular}


Table B-2. (continued)

\begin{tabular}{|c|c|c|c|}
\hline State Name & Facility Name & Units & Type \\
\hline \multirow[t]{5}{*}{ Pennsylvania } & Beaver Valley Power Station & $1 \& 2$ & PWR \\
\hline & Limerick Generating Station & $1 \& 2$ & BWR \\
\hline & Peach Bottom Atomic Power Station & $2 \& 3$ & BWR \\
\hline & Susquehanna Steam Electric Station & $1 \& 2$ & BWR \\
\hline & Three Mile Island Nuclear Station & 1 & PWR \\
\hline \multirow[t]{4}{*}{ South Carolina } & Catawba Nuclear Station & $1 \& 2$ & PWR \\
\hline & Oconee Nuclear Plant & $1,2, \& 3$ & PWR \\
\hline & Robinson Plant & 2 & PWR \\
\hline & Virgil C. Summer Nuclear Station & 1 & PWR \\
\hline Tennessee & Sequoyah Nuclear Plant & $1 \& 2$ & PWR \\
\hline \multirow[t]{2}{*}{ Texas } & South Texas Project & $1 \& 2$ & PWR \\
\hline & Comanche Peak & 1 & PWR \\
\hline Vermont & Vermont Yankee Nuclear Power Station & 1 & BWR \\
\hline \multirow[t]{2}{*}{ Virginia } & North Anna Power Station & $1 \& 2$ & PWR \\
\hline & Surry Power Station & $1 \& 2$ & PWR \\
\hline Washington & Washington Nuclear Project & 2 & BWR \\
\hline \multirow[t]{3}{*}{ Wisconsin } & Kewaunee Nuclear Power Plant & 1 & PWR \\
\hline & $\begin{array}{l}\text { La Crosse Nuclear Generating Station } \\
\text { (closed 1987) }\end{array}$ & 1 & BWR \\
\hline & Point Beach Nuclear Plant & $1 \& 2$ & PWR \\
\hline \multicolumn{4}{|c|}{ a. "World List of Nuclear Power Plants," Nuclear News, 34, 2. February 1991.} \\
\hline \multicolumn{4}{|c|}{ b. $B W R=$ boiling-water reactor. } \\
\hline \multicolumn{4}{|c|}{ c. $P W R=$ pressurized-water reactor. } \\
\hline d. HTG & reactor. & & \\
\hline
\end{tabular}


Page intentionally left blank. 



\section{APPENDIX C \\ STATE RADIATION PROGRÁĩi REPRESENTATIVES}


Page intentionally left blank.

$$
C-2
$$


Table C-1. State Radiation Program Representatives

\begin{tabular}{|c|c|}
\hline State & Draft Report Sent to \\
\hline Alabama & A. V. Godwin \\
\hline Alaska & S. D. Heidersdorf \\
\hline Arizona & C. F. Tedford \\
\hline Arkansas & G. J. Discus \\
\hline California & J. S. McGurk \\
\hline Colorado & R. M. Quillin \\
\hline Connecticut & K. T. A. McCarthy \\
\hline Delaware & A. C. Tapert \\
\hline Dist. of Columbia & F. A. Bowie \\
\hline Florida & M. E. Clark \\
\hline Georgia & T. E. Hill \\
\hline Hawaii & T. Anamizu \\
\hline Idaro & E. Ranieri \\
\hline Illinois & P. Eastvold \\
\hline Indiana & E. Wroblewski \\
\hline lowa & D. A. Flater \\
\hline Kansas & G. W. Allen \\
\hline Kentucky & D. R. Hughes \\
\hline Louisiana & W. H. Spell \\
\hline Maine & C. Toppan \\
\hline Maryland & R. G. Fletcher \\
\hline Massachusetts & R. M. Hallisey \\
\hline Michigan & G. W. Bruchmann \\
\hline
\end{tabular}




\begin{tabular}{|c|c|}
\hline State & Draft Report Sent to \\
\hline Minnesota & A. T. Dolezal \\
\hline Mississippi & E. S. Fuente \\
\hline Missouri & K. V. Miller \\
\hline Montana & A. C. Howe \\
\hline Nebraska & H. R. Borchert \\
\hline Nevada & S. R. Marshall \\
\hline New Hampshire & D. E. Tefft \\
\hline New Jersey & J. Lipoti \\
\hline New Mexico & J. Ellvinger \\
\hline New York & P. J. Merges \\
\hline North Carolina & D. H. Brown \\
\hline North Dakota & D. K. Mount \\
\hline Ohio & R. E. Owen \\
\hline Oklahoma & W. M. Kemp \\
\hline Oregon & R. D. Paris \\
\hline Pennsylvania & T. M. Gerusky \\
\hline Puerto Rico & D. Saldana \\
\hline Rhode Island & C. McMahon \\
\hline South Carolina & H. G. Shealy \\
\hline South Dakota & J. Robertson \\
\hline Tennessee & M. H. Mobley \\
\hline Texas & D. K. Lacker \\
\hline
\end{tabular}


Table C-1. (continued)

\begin{tabular}{ll}
\hline \multicolumn{1}{c}{ State } & \multicolumn{1}{c}{ Draft Report Sent to } \\
Utah & L. F. Anderson \\
Vermont & R. N. McCandless \\
Virginia & L. P. Foldesi \\
Washington & T. R. Strong \\
West Virginia & B. L. DeBord \\
Wisconsin & M. C. Bunge \\
Wyoming & J. E. Haes \\
\hline
\end{tabular}


Page intentionally left blank.

$$
\text { C-6 }
$$



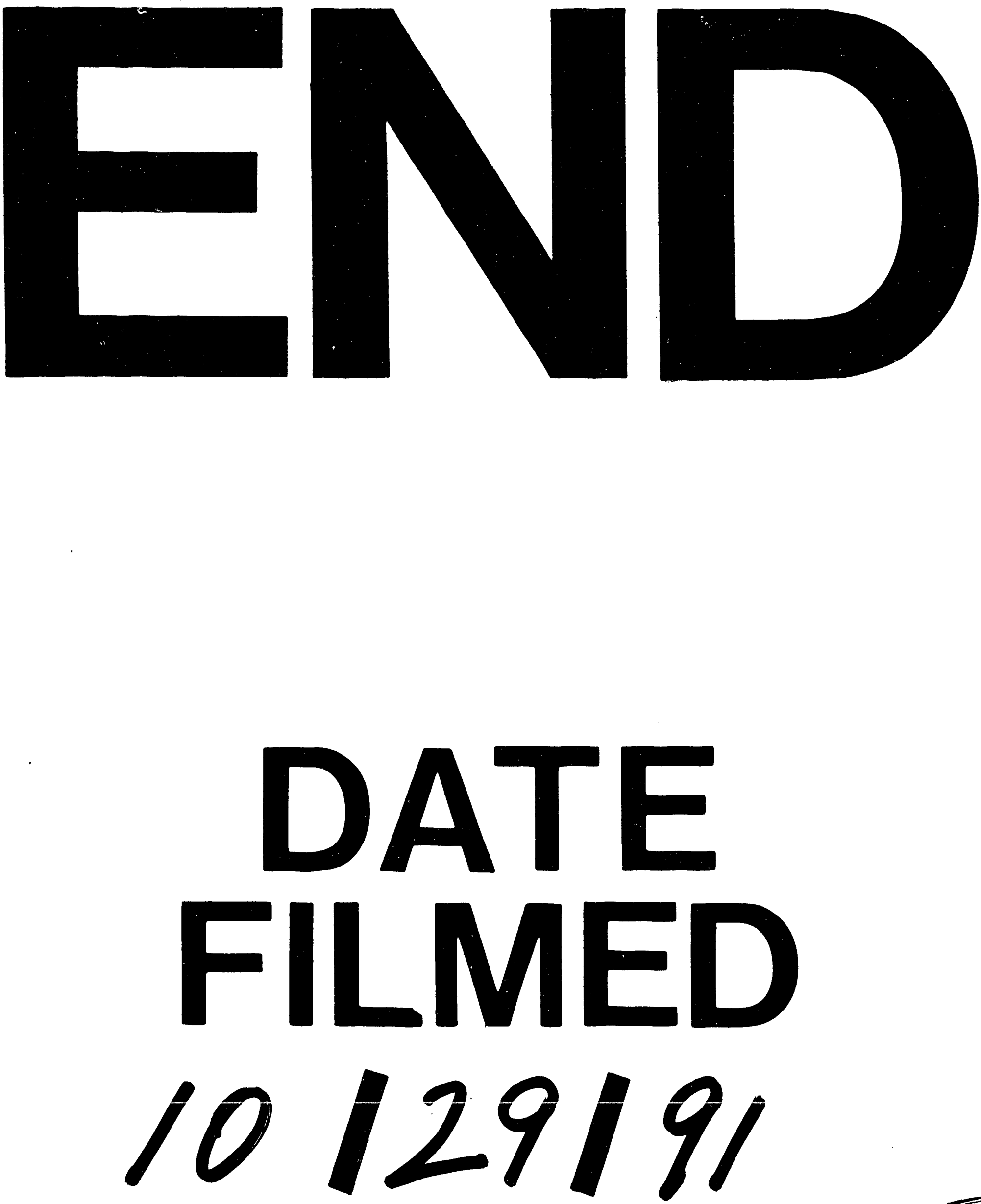

II 
